University of Rhode Island

DigitalCommons@URI

Open Access Dissertations

1995

\title{
A Transtheoretical Investigation of Immoderate Drinking in College
}

Jeffrey Peter Migneault

University of Rhode Island

Follow this and additional works at: https://digitalcommons.uri.edu/oa_diss

\section{Recommended Citation}

Migneault, Jeffrey Peter, "A Transtheoretical Investigation of Immoderate Drinking in College" (1995). Open Access Dissertations. Paper 938.

https://digitalcommons.uri.edu/oa_diss/938

This Dissertation is brought to you for free and open access by DigitalCommons@URI. It has been accepted for inclusion in Open Access Dissertations by an authorized administrator of DigitalCommons@URI. For more information, please contact digitalcommons-group@uri.edu. 


\section{A TRANSTHEORETICAL INVESTIGATION \\ OF IMMODERATE DRINKING IN COLLEGE}

BY

JEFFREY PETER MIGNEAULT

A DISSERTATION SUBMITTED IN PARTIAL FULFILLMENT OF THE REQUIREMENTS FOR THE DEGREE OF

DOCTOR OF PHILOSOPHY

IN

PSYCHOLOGY

UNIVERSITY OF RHODE ISLAND

1995 


\begin{abstract}
Immoderate alcohol use is prevalent on college campuses and results in high levels of negative consequences including injury, social, academic, and occupational disruption, increased levels of sexually transmitted disease, and emotional complaint. This study applied the Transtheoretical Model of Change to immoderate alcohol use in a cross-sectional study of 629 college students. This model has proven useful as a tool of investigation of and intervention on many other health related behaviors.

The Transtheoretical Model is a model of intentional behavior change that is composed of five interrelated multi-dimensional constructs. This investigation developed measures of four of these constructs. Two measurement approaches to the Stage of Change construct were investigated, an algorithmic five-item measure in Study I, and a continuous measure, the URICA-A, in Study II. This instrument is a three component, 17-item scale instrument. Cluster analysis was performed on scale scores to classify students into discrete stages. In Study III two solutions of the Decisional Balance construct were initially pursued, one having a unitary Cons component and the other have two separate Cons components. The final Temptation instrument as developed in Study IV was composed of four correlated subscales which in turn composed a second-order Temptation scale. The four subscales were labeled Positive/Social, Peer Pressure, Negative Affect, and Social Anxiety. The investigation of the Processes of Change in Study V resulted in an instrument measuring ten processes, including Self Monitoring, a process not previously investigated within the context of the Transtheoretical Model. Three Processes of Resistance were also hypothesized, measured, and integrated with the Processes of Change in a third-order hierarchical model. This represents a further elaboration of the Transtheoretical Model.

External validity evidence was examined for all measures by analyzing the relationships of the measurement scales to outcome variables including three alcohol consumption variables and a set of three scales, developed in this study, that assess the
\end{abstract}


negative consequences of drinking. Strong validity evidence was generally found. The relationships of the model constructs to each other were also assessed. Model variables generally showed a clear pattern across the Stages of Change as hypothesized. Disparities between hypothesized relationships and findings were generally minor and did not challenge the applicability of the model to this behavior. These findings suggest that the structural integrity of the Transtheoretical Model is preserved in this behavioral domain. The implications of these findings to intervention design and for future research are discussed. 


\section{ACKNOWLEDGMENTS}

The study of psychology is often bedeviled by the conundrum of cause and effect. I have no similar difficulty in recognizing the clear importance that many have had to my completing this Dissertation, and I would like to give my heart-felt acknowledgment to their contributions.

First is the role that the members of my committee played in both the completion of this project and my development as a psychologist. James Prochaska, as my major professor, contributed his clear thinking that challenged me to be more precise and to keep a clear view of our purpose - to understand behavior. He and Wayne Velicer also provided a work environment that was stimulating and taught me a great deal about the many facets of conducting research. John Stevenson provided me with generous time to discuss my still forming ideas, and was especially important in guiding me through my masters, my first attempt at doing psychological research. He allowed me intellectual free reign, while continually prompting me with his deep understanding of the literature. Wayne, besides being an employer, was also instrumental in my decision to become a psychologist, beginning with his encouragement of me as an extension student in his "Sadistics 300 " course while I was pondering becoming a graduate student. He has nearly single handedly taught me all I know about statistics. Barbara Brittingham has been supportive and has lent her intelligent common-sense curiosity which has enlivened my pursuit of my goals.

There are many others, without official roles, who have helped me along in important ways. Joe Fava has spent countless hours in his most patient of ways helping me more clearly understand the methods I was using and finding solutions to the difficulties I found myself in. Unto Pallonen added many hours of discussing "the model" as we tried to apply it to vocational school students.

Others on the staff of the CPRC were especially important. Lynda Hurditch was my unofficial courier, gracefully ferrying computer printouts and study drafts back and forth form 
Providence and Kingston. Elaine Taylor, who, like with everything else in the world, or at least the CPRC, helped coordinate all the pieces that needed to come together. Adrian Taylor and Julie Wellen ran countless, essential errands for me.

I also would like to acknowledge all my family members, who are too countless to mention, but helped out in a myriad of ways. I especially want to thank my son, Nicholas, whose first year of life was my last year of working on this project. He helped me keep my perspective as he tolerated my absences and cheerfully greeted me when I returned.

And lastly I cannot acknowledge fully enough the contribution of my wife, Sarah. She not only slogged through endless drafts of confused and mangled English, but also kept our lives together, was constantly encouraging, and knew when to lose her patience. 


\section{DEDICATION}

This dissertation is dedicated to my father

Roval R. Migneault

who gave me so much that he never had. 


\section{TABLE OF CONTENTS}

ABSTRACT $\ldots \ldots \ldots \ldots \ldots \ldots \ldots \ldots \ldots \ldots \ldots \ldots \ldots \ldots \ldots$ ii

ACKNOWLEDGMENTS $\ldots \ldots \ldots \ldots \ldots \ldots \ldots$ iv

DEDICATION $\ldots \ldots \ldots \ldots \ldots \ldots \ldots \ldots \ldots \ldots \ldots \ldots \ldots$ vi

\section{PART I. INTRODUCTION}

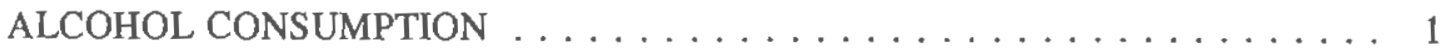

ALCOHOL RELATED PROBLEMS $\ldots \ldots \ldots \ldots \ldots \ldots \ldots \ldots \ldots$

COLLEGE STUDENT DRINKING AND ALCOHOL RELATED PROBLEMS $\ldots 5$

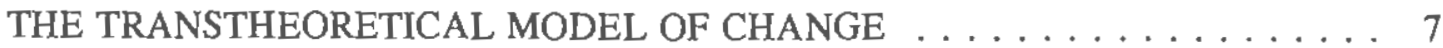

STUDY OVERVIEW . . . . . . . . . . . . . . . . 9

REFERENCES ....................... 12

\section{PART II. STUDY 1: ALGORITHMIC STAGES OF CHANGE FOR IMMODERATE}

ALCOHOL USE

INTRODUCTION ....................... 15

RESEARCH ON STAGE OF CHANGE FOR ALCOHOL USE $\ldots \ldots \ldots \ldots \ldots, 18$

STAGE INVESTIGATION DESIGN For THE PRESENT STUdY $\ldots \ldots \ldots \ldots \ldots 21$

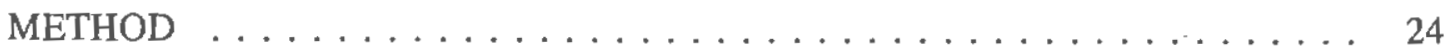

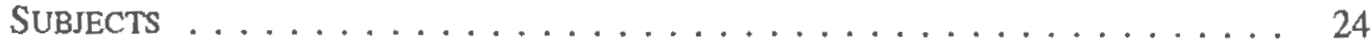

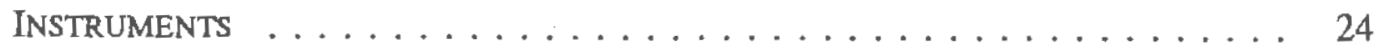

Demographic Assessment . . . . . . . . . . . . . . 25

Stages of Change: Algorithmic Assessment $\ldots \ldots \ldots \ldots \ldots \ldots .25$

Alcohol-Related Experiences . . . . . . . . . . . . . . . 26

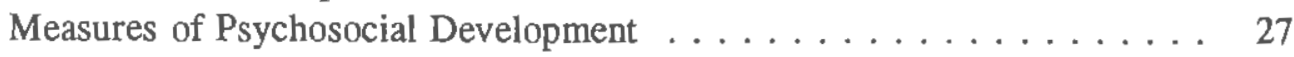

PROCEDURE . . . . . . . . . . . . . . . . . . . 28

RESULTS . . . . . . . . . . . . . . . . . . . . . . . 29

COMPARISON OF STAGING ALGORITHMS $\ldots \ldots \ldots \ldots \ldots \ldots \ldots 29$

EXTERNAL VALIDITY . . . . . . . . . . . . . . . . 30

Development of the Alcohol-Related Experiences instrument . . . . . 32

Stages of Acquisition . . . . . . . . . . . . . . . 33

Stages of Cessation ...................... 37

DISCUSSION . . . . . . . . . . . . . . . . . . . 41

CRITERIA FOR STAGING FOR IMMODERATE ALCOHOL UsE . . . . . . . 41

StAGE DistRIBUTtONS $\ldots \ldots \ldots \ldots \ldots \ldots \ldots \ldots \ldots \ldots . \ldots \ldots$

Stages of Cessation ..................... 43

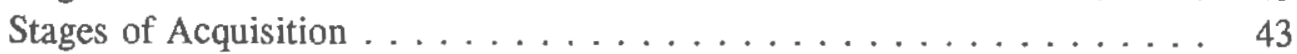

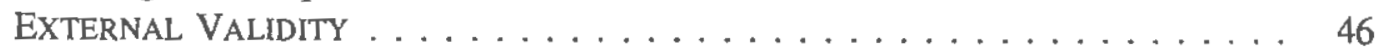

Stages of Cessation ...................... 47

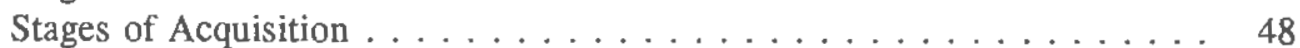

SUMMARY . . . . . . . . . . . . . . . . . 49

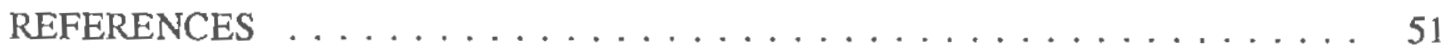


PART III. STUDY 2: URICA-A SCALE INSTRUMENT FOR MEASURING

STAGE OF CHANGE FOR IMMODERATE ALCOHOL USE

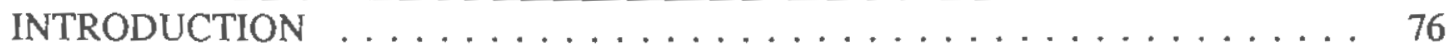

METHOD . . . . . . . . . . . . . . . . . . . . . 79

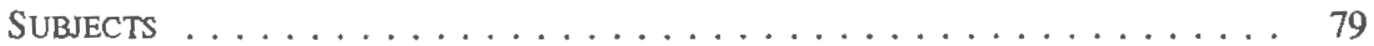

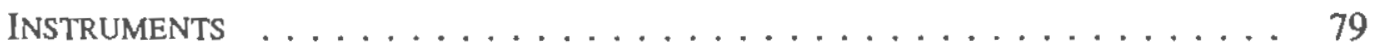

Demographic Assessment . . . . . . . . . . . . . . 80

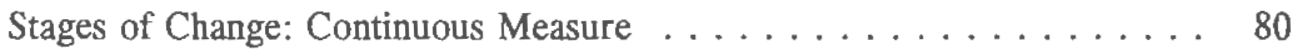

Stages of Change: Algorithmic Assessment . . . . . . . . . . 81

Alcohol Related Experiences . . . . . . . . . . . . . . . . 81

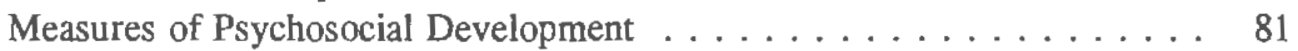

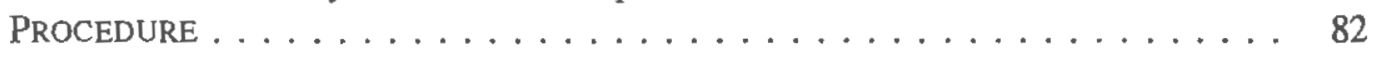

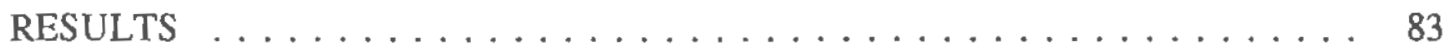

DeVElopment of a SCAle Measure $\ldots \ldots \ldots \ldots \ldots \ldots \ldots \ldots .63$

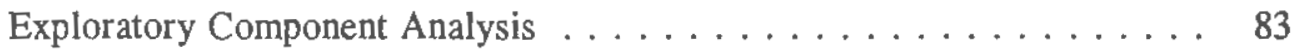

Confirmatory Factor Analysis $\ldots \ldots \ldots \ldots \ldots \ldots \ldots \ldots . \ldots . \ldots 4$

Cluster Analysis . . . . . . . . . . . . . . . . . 85

RELATIONSHIP BETWEEN ALGORITHMIC STAGES AND URICA-A CluSTERS . . 88

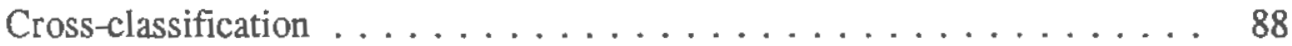

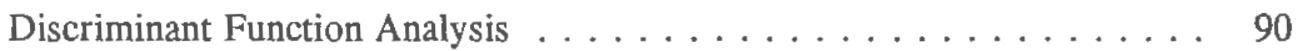

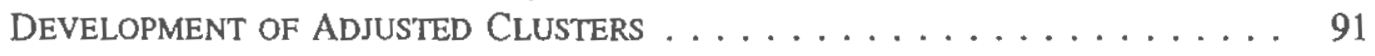

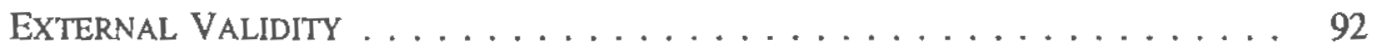

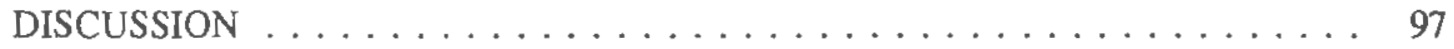

THE URICA-A . . . . . . . . . . . . . . . . . . 97

THe Clusters and AdJusted Clusters $\ldots \ldots \ldots \ldots \ldots \ldots \ldots . . \ldots 8$

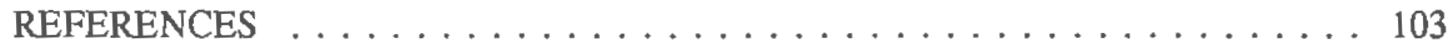

\section{PART IV. STUDY 3: DECISIONAL BALANCE FOR ALCOHOL USE}

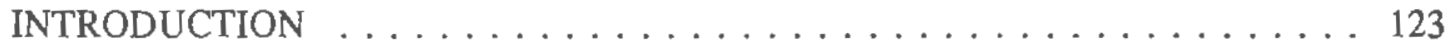

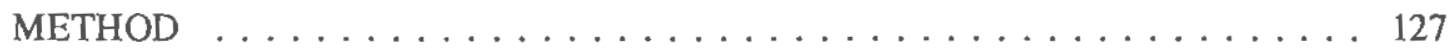

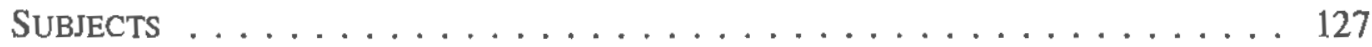

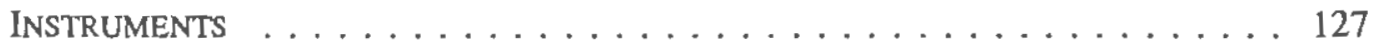

Demographics . . . . . . . . . . . . . . . . 128

Decisional Balance Item Set $\ldots \ldots \ldots \ldots \ldots \ldots \ldots \ldots \ldots \ldots$

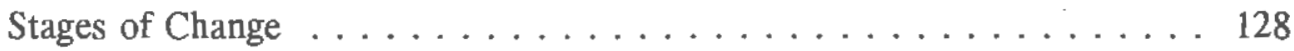

Alcohol-Related Experiences . . . . . . . . . . . . . . . . 129

PROCEDURE . . . . . . . . . . . . . . . . . . . . 130

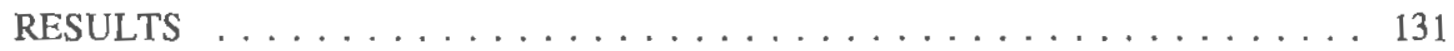

EXPLORATORY COMPONENT ANALYSIS $\ldots \ldots \ldots \ldots \ldots \ldots \ldots \ldots \ldots \ldots$

CONFIRMATORY FACTOR ANALYSIS $\ldots \ldots \ldots \ldots \ldots \ldots \ldots \ldots \ldots . \ldots \ldots$

EXTERNAL VALIDITY . . . . . . . . . . . . . . . . 134

Relationship of Decisional Balance with the Stages of Cessation . . . . 135

DISCUSSION . . . . . . . . . . . . . . . . . . . . . . . . 139

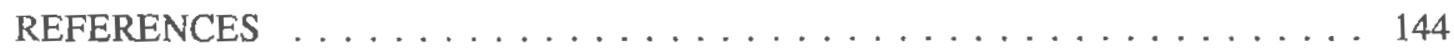


PART V. STUDY 4: TEMPTATIONS FOR IMMODERATE ALCOHOL USE

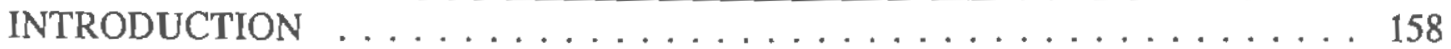

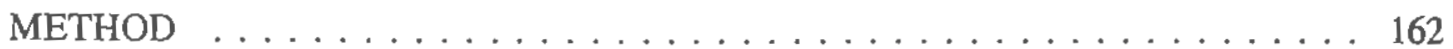

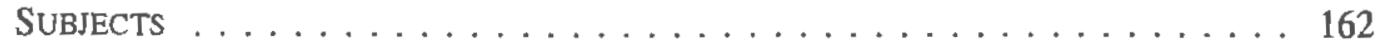

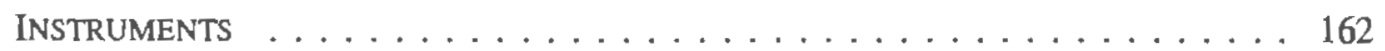

Demographics . . . . . . . . . . . . . . . . . 163

Temptation Item Set $\ldots \ldots \ldots \ldots \ldots \ldots \ldots \ldots \ldots \ldots . \ldots \ldots$

Decisional Balance Item Set . . . . . . . . . . . . . . 164

Alcohol-Related Experiences . . . . . . . . . . . . . . . . 164

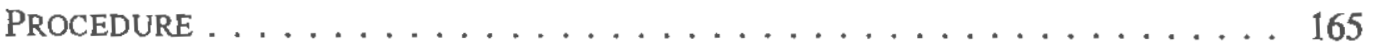

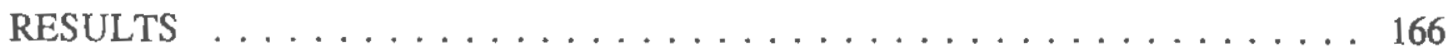

EXPLORATORY COMPONENT ANALYSIS $\ldots \ldots \ldots \ldots \ldots \ldots \ldots \ldots$

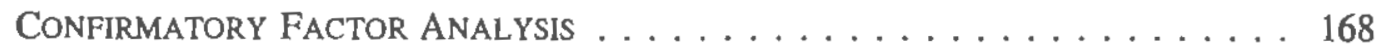

EXTERNAL VALIDITY . . . . . . . . . . . . . . . . . . . . 169

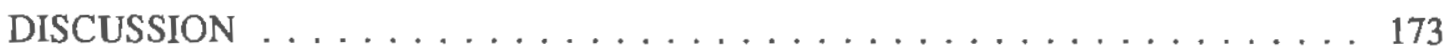

REFERENCES . . . . . . . . . . . . . . . . 177

\section{PART VI. STUDY 5: PROCESSES OF CHANGE AND RESISTANCE FOR \\ IMMODERATE ALCOHOL USE}

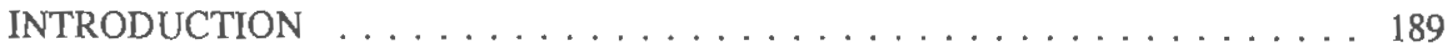

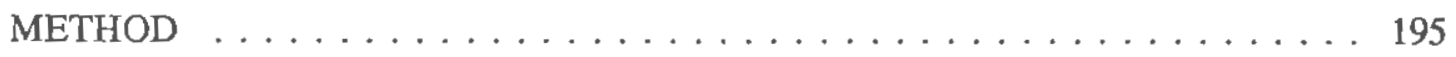

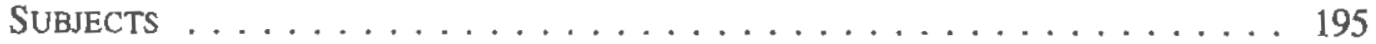

INSTRUMENTS . . . . . . . . . . . . . . . . . . . . . . 195

Demographics . . . . . . . . . . . . . . . . . . 196

Processes of Change and Resistance Item Set . . . . . . . . . 196

Stages of Change . . . . . . . . . . . . . . . . . 197

Decisional Balance Scales . . . . . . . . . . . . . . . . . 197

Temptation Scale . . . . . . . . . . . . . . . . . . 198

Alcohol-Related Experiences . . . . . . . . . . . . . . . . . 198

Procedure . . . . . . . . . . . . . . . . . . . . . . . 198

ANALYTIC PLAN . . . . . . . . . . . . . . . . . . . 199

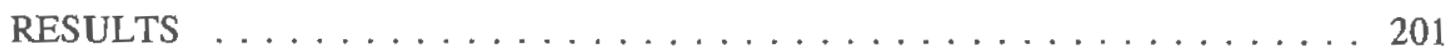

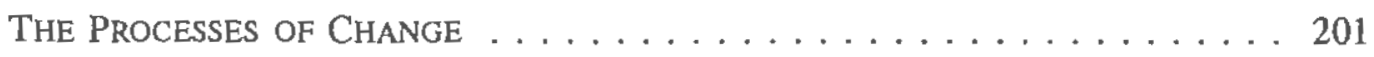

Initial Exploratory Structurai Modeling . . . . . . . . . . . 201

Process of Change Hierarchical Models . . . . . . . . . . . . 204

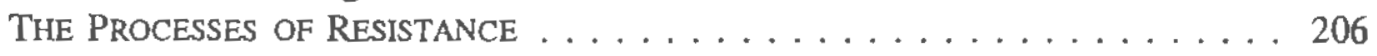

Exploratory Factor Analysis . . . . . . . . . . . . 206

Confirmatory Factor Analysis . . . . . . . . . . . . . 207

Combined Processes of Change and Processes of Resistance Models . 208

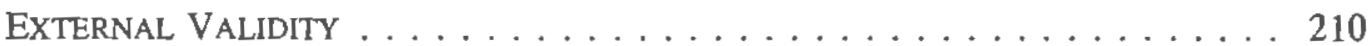

Continuous Variables . . . . . . . . . . . . . . 211

Stage of Change . . . . . . . . . . . . . . . 213

Stage of Change with Intentional Criteria for Action and Maintenance . . 215

Gender and Process use . . . . . . . . . . . . . . . . 218

DISCUSSION . . . . . . . . . . . . . . . . . . . . . . . . . 219

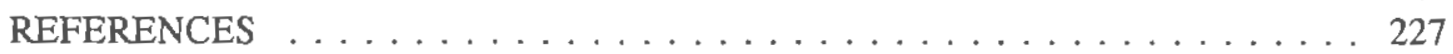


PART VII. OVERALL DISCUSSION

SCALE AND MODEL DEVELOPMENT $\ldots \ldots \ldots \ldots \ldots \ldots \ldots . \ldots \ldots 9$

COLLEGE DRINKING . . . . . . . . . . . . . . . . 271

TRANSTHEORETICAL MODEL BASED INTERVENTIONS $\ldots \ldots \ldots \ldots .273$

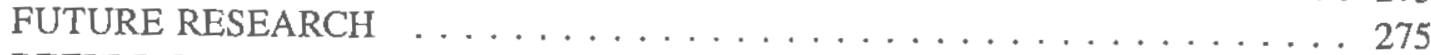

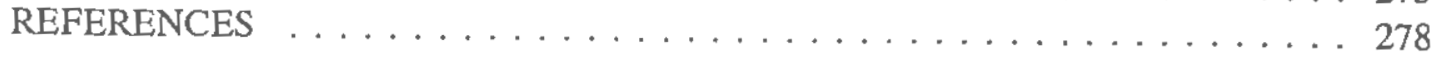

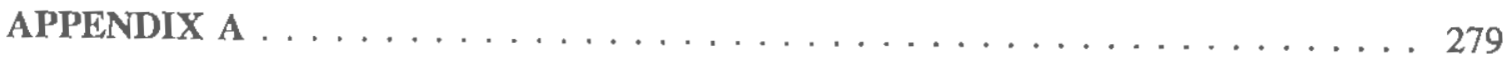

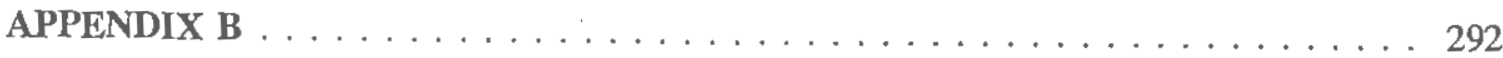

APPENDIX C . . . . . . . . . . . . . . . . . . . . . 294

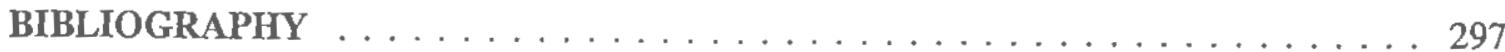




\section{LIST OF TABLES}

Table Page

2-1: Algorithm to assign Stage of Change $\ldots \ldots \ldots \ldots \ldots \ldots \ldots$

2-2: Stage distributions by criteria $\ldots \ldots \ldots \ldots \ldots \ldots \ldots \ldots \ldots \ldots \ldots$

2-3: Cross-classification of stage using different consumption criteria by gender $\ldots .58$

2-4: Final gender-specific stage distributions $\ldots \ldots \ldots \ldots \ldots \ldots \ldots \ldots$

2-5: Component loadings for Alcohol Related Experiences scales $\ldots \ldots \ldots \ldots 60$

2-6: Means, standard deviations, coefficient alphas and scale intercorrelations for the Alcohol-Related Experiences Scales . . . . . . . . . . . . . . 61

2-7: Distributions of categorical variables across the Stages of Acquisition $\ldots \ldots 62$

2-8: Means and standard deviations for continuous variables across the Stages of

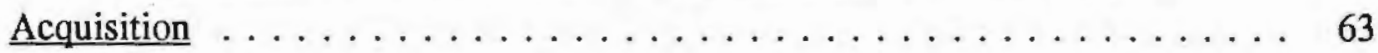

2-9: Distributions of categorical variables across Stage of Cessation ... . . . . 64

2-10: Means and standard deviations for continuous variables across the Stages of

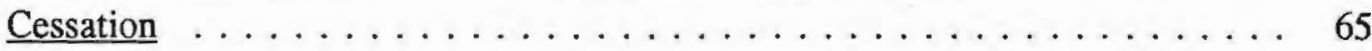

3-1: Principal component loadings for exploratory analysis of URICA-A items . . . . 106

3-2: Means, standard deviations, coefficient alphas, and scale intercorrelations for the URICA-A scales for both samples . . . . . . . . . . . . . . 107

3-3: Cross-classification of Stage and Cluster . . . . . . . . . . . . . 108

3-4: Structural Coefficients for Discriminant Function Analysis . . . . . . . . . . 109

3-5: Discriminant Function Analysis: Cross-classification matrix by jackknifed

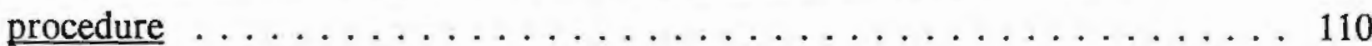

3-6: Distributions of categorical variables across Adjusted Clusters . . . . . . . 111

3-7: Means and standard deviations for continuous variables across the Adjusted

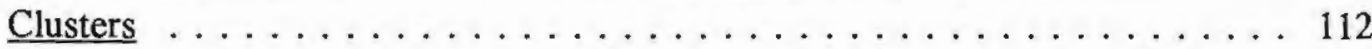

4-1: Principal component loadings for exploratory analysis of Decisional Balance items - Two component solution . . . . . . . . . . . . . . . 147

4-2: Principal component loadings for exploratory analysis of Decisional Balance items - Three component solution . . . . . . . . . . . . . . 148

4-3: Means, standard deviations, coefficient alphas and scale intercorrelations for the Decisional Balance scales for both samples . . . . . . . . . . . . 149

4-4: Correlation of Decisional Balance scales with external validity variables . . . . 150

4-5: Means and standard deviations for Decisional Balance scales by the Stages of Cessation . . . . . . . . . . . . . . . . . . . 151

4-6: Means and standard deviations for Decisional Balance scales by Adjusted 
5-1: Principal component loadings for exploratory analysis of Temptation items $\ldots . .181$

5-2: Means, standard deviations, coefficient alphas and scale intercorrelations for the Temptation full scale and subscales for both samples $\ldots \ldots \ldots \ldots 182$

5-3: Correlation of Temptation full scale and subscales with external validity variables $\ldots \ldots \ldots \ldots \ldots \ldots \ldots \ldots \ldots \ldots \ldots \ldots \ldots \ldots$

5-4: Means and standard deviations for Temptation scales by the Stages of Cessation . . . . . . . . . . . . . . . . . . . . 184

6-1: Processes of Change and Resistance definitions $\ldots \ldots \ldots \ldots \ldots \ldots \ldots 231$

6-2: Factor correlations from Correlated Processes of Change structural model . . . 232

6-3: Means, standard deviations, coefficient alphas for the Processes of Change

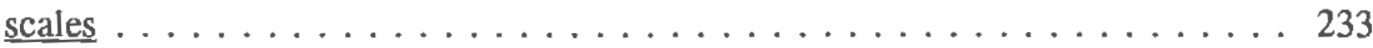

6-4: Inter-scale correlations for 10 Processes of Change $\ldots \ldots \ldots \ldots \ldots . \ldots 234$

6-5: Item factor loadings for Hierarchical Structural Model . . . . . . . . . . . 235

6-6: Exploratory component analysis loadings for reduced set of Processes of Resistance items . . . . . . . . . . . . . . . . . . . . 237

6-7: Means, standard deviations, coefficient alphas and scale intercorrelations for the Processes of Resistance scales $\ldots \ldots \ldots \ldots \ldots \ldots \ldots \ldots \ldots$

6-8: Scale correlations for the Processes of Change with the Processes of Resistance . . . . . . . . . . . . . . . . . . . . . . . . . 239

6-9: Correlations of Processes of Change and Resistance with validity variables . . . 240

6-10: Correlations of Processes of Change and Resistance with Transtheoretical

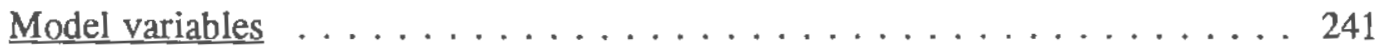

6-11: Means, standard deviations, and ANOVA results for Processes by the Stages of Cessation . . . . . . . . . . . . . . . . . . 242

6-12: Means, standard deviations, and ANOVA results for Processes by the Stages with intentional criteria for Action and Maintenance . . . . . . . . . . 243

6-13: Summary of Processes of Change and Resistance by Stage . . . . . . . 244

6-14: Means, standard deviations, and ANOVA results for Processes by gender . . . . 245 


\section{LIST OF FIGURES}

Figure Page

2-1: Distribution of Days by gender $\ldots \ldots \ldots \ldots \ldots \ldots \ldots \ldots \ldots \ldots \ldots \ldots \ldots . \ldots \ldots$

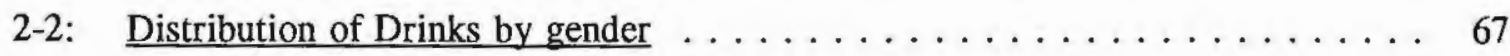

2-3: Age by Stage of Acquisition $\ldots \ldots \ldots \ldots \ldots \ldots$

2-4: Consumption variables by Stage of Acquisition . . . . . . . . . . . 69

2-5: Alcohol-Related Experiences scales by Stage of Acquisition $\ldots \ldots \ldots 70$

2-6: Psychosocial development scales by Stage of Acquisition $\ldots \ldots \ldots . \ldots 71$

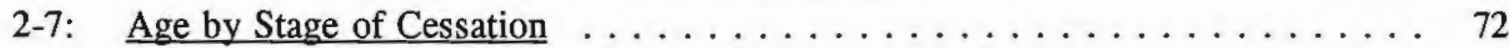

2-8: Consumption variables by Stage of Cessation . . . . . . . . . . . . 73

2-9: Alcohol-Related Experiences scales by Stage of Cessation . . . . . . . . . . 74

2-10: Psychosocial development scales by Stage of Cessation $\ldots \ldots \ldots \ldots$

3-1: Structural model for URICA-A scales . . . . . . . . . . . . . . 113

3-2: Profiles of PC-1 and PC-2 URICA-A clusters $\ldots \ldots \ldots \ldots \ldots 14$

3-3: $\quad$ Profiles of Immotive-PC and Immotive-M URICA-A clusters $\ldots \ldots \ldots 115$

3-4: $\quad$ Profiles of Contemplation and Preparation URICA-A clusters $\ldots \ldots \ldots 116$

3-5: Profiles of Action and Maintenance URICA-A clusters . . . . . . . . . 117

3-6: Group centroids for discriminant functions $\ldots \ldots \ldots \ldots \ldots \ldots$

3-7: Age by Adjusted Cluster . . . . . . . . . . . . . . . . . 119

3-8: Consumption variables by Adjusted Clusters . . . . . . . . . . . . . . 120

3-9: Alcohol-Related Experiences scales by Adjusted Cluster . . . . . . . . . . . 121

3-10: Psychosocial development scales by Adjusted Cluster . . . . . . . . . 122

4-I: Two factor structural model for Decisional Balance scales . . . . . . . . . 153

4-2: Three factor structural model for Decisional Balance Scales . . . . . . . . . . 154

4-3: Hierarchical structural model for Decisional Balance scales . . . . . . . . . . 155

4-4: Decisional Balance scales by Stage of Change . . . . . . . . . . . . . 156

4-5: Decisional Balance scales by Adjusted Cluster . . . . . . . . . . . . . 157

5-1: Correlated structural model for Temptations scales . . . . . . . . . . . 185

5-2: Hierarchical structural model for Temptations scales $\ldots \ldots \ldots \ldots$

5-3: Full Temptation scale by Stage of Cessation $\ldots \ldots \ldots \ldots$. . . . . . . 187

5-4: Temptation subscales by Stage of Cessation . . . . . . . . . . . . . . 188

6-I: Item loadings for the 10 Processes of the Correlated Structural Model . . . . . 246

6-2: Factor loadings for the Two-Factor Hierarchical Structural Model . . . . . . 249

6-3: Structural model for three Processes of Resistance scales . . . . . . . . . . 250

6-4: Structural model for two Processes of Resistance scales . . . . . . . . . . . . 251

6-5: Correlated Hierarchical Model for the Processes of Change and Resistance . . . 252 
6-6: Third-order Hierarchical Model for the Processes of Change and Resistance . . 253

6-7: Environmental Reevaluation and Consciousness Raising by Stage of Cessation . . 254

6-8: Dramatic Relief and Self Liberation by Stage of Cessation . . . . . . . . 255

6-9: Stimulus Control and Self Reevaluation by Stage of Cessation . . . . . . . 256

6-10: Reinforcement Management and Counter-Conditioning by Stage of Cessation . . 257

6-11: Helping Relationship and Self Monitoring by Stage of Cessation . . . . . 258

6-12: Repression/Denial, Reactance, and Rationalization by Stage of Cessation . . . 259

6-13: Environmental Reevaluation and Consciousness Raising by Stage with intentional criterion for Action and Maintenance $\ldots \ldots \ldots \ldots . \ldots 260$

6-14: Dramatic Relief and Self Liberation by Stage with intentional criterion for Action and Maintenance $\ldots \ldots \ldots \ldots$. . . . . . . . . . . . . . 261

6-15: Stimulus Control and Self Reevaluation by Stage with intentional criterion for

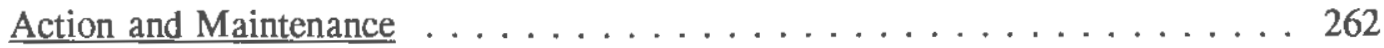

6-16: Reinforcement Management and Counter Conditioning by Stage with intentional criterion for Action and Maintenance . . . . . . . . . 263

6-17: Helping Relationship and Self Monitoring by Stage with intentional criterion for Action and Maintenance . . . . . . . . . . . . . . . 264

6-18: Repression/Denial, Reactance, and Rationalization by Stage with intentional criterion for Action and Maintenance $\ldots \ldots \ldots \ldots \ldots \ldots \ldots \ldots$

6-19: Reinforcement Management, Stimulus Control. Self Liberation, and Rationalization by Stage of Cessation . . . . . . . . . . . 266

6-20: Environmental Reevaluation, Consciousness Raising, Self Reevaluation, and Rationalization by Stage of Cessation . . . . . . . . . . . . 267

6-21: Self Monitoring. Counter Conditioning. Dramatic Relief, and Rationalization by Stage of Cessation . . . . . . . . . . . . . . . . . . 268

C-1: Decisional Balance by Stage of Change . . . . . . . . . . . . . 294

C-2: Decisional Balance scales by Adjusted Cluster . . . . . . . . . . . . 295 


\section{PART I. INTRODUCTION}

Zinberg (1981) suggested that in addition to Freud's categories of work and love, success in the areas of religion, food, and psychoactive substance use was necessary for success in life. This view suggests that the challenge of managing the use of psychoactive substances exists in everyone's life, not just in the lives of addicts who are so often focused on. Although in our society caffeine is the most widely used psychoactive substance, it is the intake of alcohol more than any other substance that the greatest number of people must learn how to control or suffer significant negative consequences.

The majority of individuals do eventually attain a controlled and relatively safe pattern of alcohol use, although a significant minority do not. Yet, even of those who do attain safe consumption patterns, most go through a period of heavy or immoderate alcohol use in adolescence or early adulthood. This period puts them at much higher risk for a set of acute negative consequences of alcohol use, such as accidental injury, scholastic failure, and social and developmental disruption. The cost of this time-limited period of excessive use can be very high and can negatively affect the rest of their lives.

Greater understanding of the development of safe consumption patterns after a period of excessive use would allow for the development of informed interventions to reduce the prevalence of acute alcohol related problems, while also promoting the development of safe alcohol consumption patterns. The present study will attempt to further this endeavor.

\section{ALCOHOL CONSUMPTION}

Most Americans progress through a period that entails some risky drinking in late adolescence or young adulthood before developing safer alcohol consumption patterns. Harford (1984) found a curvilinear pattern of drinking habits with age, with younger and older individuals tending to drink smaller amounts at home, whereas older adolescents and young adults tend to drink in public and at much higher levels. The extensive annual survey 
of a longitudinal sample, Johnston, O'Malley and Bachman (1992) found that the two week prevalence of drinking five or more drinks in a row peaks at $40 \%$ for $21-22$ year olds. By age thirty this rate was $24 \%$.

Despite the modal pattern for Americans being a period of excessive drinking followed by controlled drinking there is much variety in life-long drinking patterns. Substantial groups of individuals include life-long abstainers, those who never drink to excess, those who periodically have problems with alcohol, and those who develop into long-term alcoholic drinkers. Most surveys show that cross-sectionally about one-third of the adult population are abstaining and about one-tenth are drinking alcoholically (American Psychiatric Association, 1987). Estimates of heavy or problem drinking vary with the survey population and operational definitions. In a recent survey of 43,809 households $25 \%$ were drinking $4-13$ drinks per week and $13 \%$ were drinking 14 or more (Williams \& Debakey, 1992). Unfortunately average drinks per occasion, a more useful measure of problem drinking, was not reported.

There are large gender differences in alcohol use patterns. Women are more likely to abstain than men, those who drink are more likely to be light drinkers, and on average consume about half as much alcohol as men (Williams \& Debakey, 1992). Young women, ages 18-32, have one-half the two-week prevalence of drinking five or more drinks of young men (Johnston et al., 1992). Yet these statistics overstate the gender difference. Alcohol is known to have a greater physiological effect on women than men. Recent research has explored the mechanisms of this difference and quantified the effects (Frezza, de Padova, Pozzato, Terpin, Baraona, \& Lieber, 1990). It is estimated that the $100 \%$ greater consumption of alcohol by men represents a $38 \%$ greater physiological alcohol effect (Dawson \& Archer, 1992). 


\section{ALCOHOL RELATED PROBLEMS}

The costs to society of alcohol consumption are great. It is estimated that there are approximately 100,000 deaths annually attributable to alcohol use (Moskowitz, 1989), and that $15 \%$ of the national health care expenditure is related to alcohol consumption (Science 1987). Other costs include lost productivity of those impaired by alcohol and the untold psychological damage caused to those abusing alcohol and to those with whom they have intimate ties.

The negative consequences of alcohol use can be classified as either the result of acute or chronic processes (Babor, Kranzler, \& Lauerman, 1987). For instance, some alcohol related deaths are caused by chronic conditions such as cirrhosis of the liver and heart disease, and others by acute processes such as automobile accidents. Other acute negative consequences of alcohol include other types of accidents, violence towards others, suicide, crime and crime victimization, and sexually transmitted disease and unwanted pregnancies. Other consequences can vary from chronic to acute depending on the circumstances, including such consequences as the reduction of levels of functioning at home, work or school and the disruption of intimate relationships.

The likelihood of suffering both chronic and acute negative consequences of alcohol consumption is related to blood alcohol concentrations (BAC) (Babor et al., 1987). These problems are usually attributable to one of two alcohol related effects: the reduction in ability to function and increased disinhibition. A third not fully understood effect is changes in emotional functioning caused by alcohol consumption. Acute negative consequences are found to be more likely while BAC are rising, rather than falling (Babor et al., 1987).

In contrast to these findings that emphasize the physiological effects of alcohol are those that suggest that most of the effects of alcohol are mediated by expectancies of its effects. This research comes from both cross-cultural studies and laboratory experiments (MacAndrew \& Edgerton, 1969; Marlatt \& Rohsenow, 1980). Another factor seems to be experience with intoxication, with the inexperienced drinker suffering greater rates of negative consequences (Fillmore \& Midanik, 1984). The relative effects of psychological and 
physiological processes and their interactions on the occurrence of alcohol related problems have not been fully explored.

Although the problems suffered by alcohol dependent persons and those whom they affect are well documented, the majority of the acute problems related to alcohol use are experienced by individuals who would not meet traditional definitions of alcoholism, but rather are non-dependent individuals who are occasionally intoxicated. Although not alcoholic, these individuals have not developed a pattern of alcohol consumption that is free of risk (Fingarette, 1988).

Most surveys show that it is young men who have the highest incidence of alcohol related problems and that problem drinking can be considered "normative" for youth (Fillmore \& Midanik, 1984). Furthermore it is found that drinking problems are less correlated with each other for young men than for older men. Fillmore and Midanik (1984) suggest that for young men the negative consequences of alcohol should by considered "events" rather than signs of a condition, as might be more appropriate in older age groups. Yet these events can have lasting negative impacts.

Survey results have shown that women have much lower rates of alcohol-related problems, which parallels their lower levels of consumption. There might be additional explanations for this result. Most problem lists are dominated by observable negative behaviors such as accidents, property damage, and interpersonal violence. These behaviors are in general more common in men. As alcohol behavior is strongly affected by social mores (MacAndrew \& Edgerton, 1969), women might be less likely to act out and instead may suffer other less visible negative consequences such as depression or loneliness (Berkowitz \& Perkins, 1986). Furthermore these effects might be less likely to be attributed to alcohol intake than behaviors that have a clear association with intoxication.

Problem drinking has high rates of remission in the young, and lower rates for older adults (Fillmore \& Midanik, 1984). Again there is a gender difference, with women having lower remission rates than men (Fillmore, 1974). 


\section{COLLEGE STUDENT DRINKING AND ALCOHOL RELATED PROBLEMS}

As this study will use a sample of college students, a brief description of the use and abuse of alcohol by college students is presented below. College students differ from their same-age non-college counterparts and have been much more intensively researched.

A very high percentage of college students drink. These figures have typically been in the 80 to $96 \%$ range since the early 1970's depending on the sample and definitions of drinking (Kraft, 1988). Furthermore they drink heavily. Stevenson, Migneault, and Mitchell (1990) found that the median amount drunk by undergraduate survey respondents was 4 drinks per drinking occasion and that 6 was the median number of days per month that alcohol was consumed. Moreover $28 \%$ of the undergraduates drank 6 or more drinks per occasion, and the same percentage (28\%) drank on 9 or more days per month. Wechsler and McFadden (1979) found similar numbers of heavy drinkers.

Although college students do not have higher lifetime prevalence of alcohol use than young non-college adults, they are higher on thirty-day prevalence and on measures of heavy drinking (Johnston et al., 1992). College students have a 2-week prevalence of heavy drinking of $42.8 \%$ whereas the figure for the same age non-college young adults is $34.4 \%$. This difference has developed over the last five years, with non-college young adults drinking less heavily each of these years, whereas college students' drinking patterns have not changed appreciably in this time. This study also showed large gender differences, with the heavy drinking prevalence for men and women as $52.3 \%$ and $34.9 \%$ respectively (Johnston et al, 1992).

It is also been observed that the typical drinking pattern for college students is light or no drinking during the week, and very heavy drinking on Friday and Saturday nights and that this is the pattern that needs to be explained and taken into account when measuring college drinking (Baer, Stacy, \& Larimer, 1991). In a preliminary survey (Migneault, unpublished data), it was found that Introductory Psychology students who drink, drink on average 4-5 
drinks on Friday and Saturday, 2 drinks on Thursday, and only about 0.30 on Sunday through Wednesday.

Changes observed over the four years of college parallel the changes observed in the general population. As students progress in college they are less likely to abstain, more likely to drink smaller amounts, and to drink more frequently (Engs, 1977; Stevenson, et al., 1990). They also are more likely to drink in smaller groups or dyads, and in private settings (Harford, 1984).

College students also experience high rates of alcohol-related problems. In a nationally representative sample of college students, Engs (1977) found that $51 \%$ of students had experienced one to four alcohol-related problems in the previous year. More recently a study of students at a small private university in the northeast found that in the previous week $25 \%$ of students reported having a hangover, $7.5 \%$ had vomited, $4 \%$ had had a blackout (Meilman, Stone, Gaylor, \& Turco, 1990). Again paralleling the general population, women experience fewer problems than men (Berkowitz \& Perkins, 1986).

In summary, a period of problem drinking with associated acute risks followed by the development of lifelong controlled and safe drinking is the norm, although significant numbers either do not enter this process or do not successfully complete it. College students, the target population of this study, should provide rich data with which to explore this phenomenon. This population is on average more extreme than their same age non-college counterparts, and they experience very high rates of negative consequences. Their ages span the years of peak heavy drinking and the first years of increasing control which will eventually lead to safe drinking habits for the majority. Increased understanding of this modal or "normal" path of developing controlled or moderate drinking will eventually lead to new policies and interventions to enhance the development of safe drinking patterns and to minimize risks during this developmental period. 


\section{THE TRANSTHEORETICAL MODEL OF CHANGE}

To investigate the cessation of immoderate alcohol use and the development of controlled and moderate consumption patterns this study will use an integrative model of intentional behavior change, the Transtheoretical Model of Change. This model has received increasing attention since its inception (Prochaska, 1979). This attention has been fueled by the continuing development of the model both conceptually and empirically, and by its successful application to a very wide range of behaviors and populations. Yet the model's application to the behavior of alcohol use has been of limited scope and generalizability. Using the Transtheoretical Model to investigate the intentional efforts of college students to control their alcohol use will constitute a. new perspective on alcohol consumption, and promises to provide new understandings.

The Transtheoretical Model uses a process focus to explain behavior and behavior change. The Processes of Change are a set of overt and covert processes that people use to change their affect, thinking, relationships, or behavior. The original set of processes were delineated within an exhaustive study of 18 major schools of psychotherapy (Prochaska, 1979). This integration of developed therapeutic models provides the conceptual backbone of the model.

Since its inception, the Transtheoretical Model has been further developed, and four other constructs have been added to the Processes of Change. Each of these has received further conceptual and empirical development. In brief, the model postulates three major dimensions to the structure of change. The Processes of Change, mentioned above represent how an individual modifies his or her behavior. A second dimension, the Stages of Change, represents a motivational stance and delineates when a person uses the processes to change behavior. The third dimension, the Levels of Change, represents what the content of the change is and includes five levels: symptoms/situations, cognitions, interpersonal relationships, family and systems, and the intrapersonal (Prochaska \& DiClemente, 1984). In addition to these three dimensions the model has incorporated two other constructs taken from 
other models of behavior. Decisional Balance refers to a decision making construct developed from the conflict theory of Janis and Mann (1977) (Velicer, DiClemente, Prochaska, \& Brandenburg, 1985). Self Efficacy (or alternatively Temptation) is a measure based on the work of Bandura $(1977,1982)$ and represents another determinant of behavior (DiClemente, Prochaska, \& Gilbertini, 1985). Both of these constructs have been integrated into the model, and have been widely applied. The expected cross-sectional and Iongitudinal relationships between the Stages of Change and these constructs have been specified and empirically validated.

The Transtheoretical Model can be applied to most types of intentional behavior change. It can be conceptualized for acquisition or cessation of both positive and negative behaviors. Most empirical research has been on the cessation of negative behaviors such as cigarette use, with substantial work also being done on the acquisition of positive behaviors such as exercise. Only limited work has been completed on the acquisition of negative behaviors, and the extent of the model's explanatory power for this domain is yet to be fully determined. The cessation of positive behaviors, or one type of relapse, has not received substantial attention.

The research to date using the Transtheoretical Model across a wide range of behaviors and populations has produced a range of variations in model findings that are consistent with the conceptual structure of the model and provide useful information about the specific change process used to change the behavior in question by the population studied. These results provide a rich context in which to interpret new findings. Furthermore, research providing new deviations in model predictions, if not so extreme as to invalidate the model provides useful information about how a behavioral domain is unique from other studied domains.

All in all the Transtheoretical Model represents a broad model that allows for the understanding and integration of many aspects of behavior and behavior change and for the 
prediction of future behavior. As such it provides a new paradigm with which to investigate a phenomenon such as the development of controlled alcohol use.

\section{STUDY OVERVIEW}

The present investigation studies the application of four of the five major constructs of the Transtheoretical Model of Change: Stages of Change, Decisional Balance, Temptation, and the Processes of Change. The focus will be on using the model to study the cessation of immoderate drinking although some evidence on the validity of the Stages of Acquisition will also be presented. Additionally, in an attempt to contribute to the further development of the model a new Process of Change is investigated, as are a set of Processes of Resistance. Processes of Resistance have received only minimal attention to date (Prochaska, Norcross, Fowler, Follick, \& Abrams, 1992) and have not previously been systematically integrated into the Transtheoretical Model.

The over-arching hypothesis of this study was that the Transtheoretical Model of Change will fit the data as measured by the survey administered. This general hypothesis led to a large number of other sub-hypotheses at varying levels of specificity. The validation of the model does not rest with the support of any one hypothesis, but rather with the pattern of results being largely congruent with the set of hypotheses generated. In this way, significance testing, although important, played a somewhat less important role in this study than in more traditional research. Rather, the pattern of results, and the explanatory value of the constructs as demonstrated with methodologies such as principal component analysis, discriminant function analysis, cluster analysis, and structural modeling, along with simpler graphical analysis was heavily relied upon. The following is an explication of the hypothesized results.

1) Instrument Structure.

The four instruments developed will possess a factor structure that is consistent with the construct conceptualization and will possess strong psychometric properties. 
These include instruments to measure the Stage of Change, Decisional Balance, Temptations, and the Processes of Change.

2) Stage of Change.

a) The staging algorithm will stage all students into a Stage of Cessation or a Stage of Acquisition. Precontemplation for Cessation will have the most students. There will be larger numbers in the Contemplation, Action, and Maintenance stages for cessation in the upper classes than the under classes.

b) Cluster analysis will classify most subjects into interpretable subgroups. Stage, as determined by cluster and algorithm, will be in general agreement.

c) Except for school class and age there will not be significant relationships between stage and demographic variables.

d) Orderly relationships will be found between Stage of Change and other alcohol related variables.

3) Relationships found between Transtheoretical Model constructs will support the model.

a) Stage and Decisional Balance

i) Precontemplators will score higher, using standardized scores, on the Pros of drinking than on the Cons.

ii) Students in Action and Maintenance will score higher on the Cons of drinking than the Pros.

iii) Students in Contemplation will score about the same on the Pros and Cons of drinking.

iv) The change in the Cons of drinking between Precontemplation and Contemplation or Action (whichever is higher) will be on the order of one standard deviation. The corresponding change in the Pros of drinking will be on the order of a 0.5 standard deviation decrease. 
b) Stage and Temptation

i) The total Temptation score will start out high for Precontemplators and decrease somewhat in the early stages and more sharply in Action and Maintenance.

c) Stage and Processes of Change

i) All processes will be endorsed least by Precontemplators.

ii) Experiential processes will peak in Contemplation.

iii) Behavioral processes will peak in Action or Maintenance

d) Stage and Processes of Resistance

i) Precontemplators will use the Processes of Resistance the most, with reductions with stage progression.

This investigation is separated into five studies. The first two investigate the Stages of Change, the first focusing on an algorithmic staging procedure for both the Stages of Acquisition and the Stages of Cessation and the second on a continuous measure of Stages of Cessation. The next three studies present the development of measurement scales and the investigation of their validity for Decisional Balance, Temptations, and Processes of Change and Resistance constructs in that order. External validity evidence is presented for both alcohol related variables and for model variables that have been developed. In this way, with each study, a more complete picture of the application of the Transtheoretical Model to immoderate drinking in college is presented. 


\section{REFERENCES}

American Psychiatric Association. (1987). Diagnostic and statistical manual of mental disorders (3rd ed.). Washington, DC: American Psychiatric Association.

Babor, T. F., Kranzler, H. R., \& Lauerman, R. J. (1987). Social drinking as a health and psychosocial risk factor: Anstie's limit revisited. In M. Galanter (Ed.), Recent developments in alcoholism (pp. 373-402). New York: Plenum.

Baer, J. S., Stacy, A., \& Larimer, M. (1991). Biases in the perception of drinking norms among college students. Journal of Studies on Alcohol, $\underline{52}, 580-586$.

Bandura, A. (1977). Self-efficacy: Toward a unifying theory of behavioral change. Psychological Review, 84, 191-215.

Bandura, A. (1982). Self-efficacy: Mechanism in human agency. American Psychologist, $\underline{37}$, $122-147$.

Berkowitz, A. D. \& Perkins, H. W. (1986). Problem drinking among college students: A review of recent research. College Heaith, 35, 21-28.

Dawson, D. A. \& Archer, L. (1992). Gender differences in alcohol consumption: Effects of measurement. British Journal of the Addictions, 87, 119-123.

DiClemente, C. C., Prochaska, J. O., \& Gilbertini, M. (1985). Self-efficacy and the stages of self change of smoking. Cognitive Therapy and Research, $9,181-200$.

Engs, R. (1977). Drinking patterns and drinking problems of college students. Journal of Studies on Alcohol, 38, 2144-2156.

Fillmore, K. M. (1974). Drinking and problem drinking in early adulthood and middle age. Quarterly Journal of Studies on Alcohol, 35, 819-840.

Fillmore, K. M. \& Midanik, L. (1984). Chronicity of drinking problems among men: A Iongitudinal study. Journal of Studies on Alcohol, 45, 228-236.

Fingarette, H. (1988). Heavy Drinking: The myth of alcoholism as a disease. Berkeley, CA: University of California Press. 
Frezza, M., de Padova, C., Pozzato, G., Terpin, M., Baraona, E., \& Lieber, C. S. (1990). High blood alcohol levels in women:the role of decreased gastric alcohol dehydrogenase activity and first-pass metabolism. New England Journal of Medicine, $\underline{322}, 127-129$.

Harford, T. C. (1984). Situational factors in drinking: A developmental perspective on drinking contexts. In P. M. Miller \& T. D. Nirenberg (Eds.), Prevention of alcohol abuse (pp. 119-156). New York: Plenum Press.

Janis, I. L. \& Mann, L. (1977). Decision making: A psychological analysis of conflict. choice and commitment. New York: Free Press.

Johnston, J. D., O’Malley, P. M., \& Bachman, J. G. (1992). Smoking, drinking, and illicit drug use among American secondary school students, college students, and young adults, 1975-1991 (Vol. 2). Washington, D.C.: National Institute on Drug Abuse.

Kraft, D. P. (1988). The prevention and treatment of alcohol problems on a college campus. Journal of Alcohol and Drug Education, 34, 37-51.

MacAndrew, C. \& Edgerton, R. B. (1969). Drunken comportment: A social explanation. Chicago: Aldine.

Marlatt, G. A. \& Rohsenow, D. J. (1980). Cognitive processes in alcohol use: Expectancy and the balanced placebo design. In N. K. Mello (Ed.), Advances in substance abuse: Behavioral and biological research (pp. 159-199). Greenwich, CT: JAI Press.

Meilman, P. W., Stone, J. E., Gaylor, M. S., \& Turco, J. H. (1990). Alcohol consumption by college undergraduates: Current use and 10-year trends. Journal of Studies on Alcohol, 51, 389-395.

Moskowitz, J. M. (1989). The primary prevention of alcohol problems: A critical review of the research Literature. Journal of Studies on Alcohol, 50, 54-88.

Prochaska, J. O. (1979). Systems of psychotherapy: A transtheoretical analysis (2nd ed.). Homewood. Illinois: Dorsey Press. 
Prochaska, J. \& DiClemente, C. (1984). The transtheoretical approach: Crossing the traditional boundaries of therapy. Homewood, Illinois: Dow-Jones/Irwin.

Prochaska, J. O., Norcross, J. C., Fowler, J. L., Follick, M. J., \& Abrams, D. B. (1992). Attendance \& outcome in a worksite weight control program: Processes and stages of change as process and predictor variables. Addictive Behaviors, $\underline{17}, 35-45$.

Science. (1987). Is alcoholism treatment effective? 236, 20-22.

Stevenson, J., Migneault, J. P., \& Mitchell, R. (1990, Nov). The University of Rhode Island alcohol-drug health survey: Final report. Unpublished report.

Velicer, W. F., DiClemente, C. C., Prochaska, J. O., \& Brandenburg, N. (1985). A decisional balance measure for assessing and predicting smoking status. Journal of Personality and Social Psychology, 48, 1279-1289.

Wechsler, H. \& McFadden, M. (1979). Drinking among college students in New England. Journal of Studies on Alcohol, 40 , 969-996.

Williams, G. D. \& Debakey, S. F. (1992). Changes in levels of alcohol consumption: United States, 1983-1988. British Journal of the Addictions, 87, 643-648.

Zinberg, N. E. (1981). Alcohol addiction: Toward a more comprehensive definition. In M. H. Bean \& N. E. Zinberg (Eds.), Dynamic approaches to the understanding and treatment of alcoholism. New York: The Free Press. 


\section{PART II. STUDY 1: ALGORITHMIC STAGES OF CHANGE FOR IMMODERATE ALCOHOL USE}

\section{INTRODUCTION}

Research has shown that heavy drinking is very common on college campuses across the United States and has been since the early 1970's (Wechsler \& McFadden, 1979; Kraft, 1988). In a recent study using a large representative national sample $(\mathrm{N}=17,592)$ Wechsler, Davenport, Dowdall, Moeykens, and Castillo (1994) found that $44 \%$ of college students binge drank in the previous two weeks. This pattern of drinking has associated with it high levels of negative consequences including accidental injury, interpersonal harm, sexually transmitted disease, scholastic and occupational failure, and developmental disruptions (Berkowitz \& Perkins, 1986; Engs, 1977; Meilman, Stone, Gaylor, \& Turco, 1990; Wechsler et al. 1994). Efforts to change alcohol consumption patterns in college and reduce these negative consequences have been disappointing, and the field is open to new approaches (Kraft, 1988).

The Transtheoretical Model of Change has not been substantially applied to college drinking and represents a new approach to this behavioral domain. This model has as its organizing construct the Stage of Change. This construct is composed of a set of stages that individuals progress through when intentionally changing their behavior (Prochaska \& DiClemente, 1984). Individuals progress through the stages in an ordered, but often cyclical pattern, with repeated regressions to earlier stages being expected for many individuals before they attain long term behavior change (Prochaska \& DiClemente, 1983, 1984). The stages form a simplex pattern, with each stage more closely related to adjacent stages than to distant ones (Prochaska, DiClemente, Velicer, Ginpil, \& Norcross, 1985), and possess a stability that is between that of states and traits. 
Originally four stages were conceptualized and measured. Individuals were either in Precontemplation (having no intention to change the target behavior), in Contemplation (expressing an intention to change in the near future, usually defined as the next six months), in Action (having recently changed the behavior, usually within the last six months), or Maintenance (having made the change more than six months ago but still actively resisting relapse). Subsequently two other stages, Preparation and Termination have been conceptualized and empirically supported. Preparation can be seen as a subset of Contemplation and includes individuals who have made a strong commitment to change, usually within the next 30 days and usually have also made some attempts at behavioral change. Those in Termination have completed the change process and no longer need to expend effort to prevent relapse (Prochaska \& DiClemente, 1984).

The stage variable is the most widely used construct of the model. It is easily adaptable to a wide range of behaviors, and is usually the first Transtheoretical Model construct investigated for a behavior. It is an important variable as it integrates a number of attributes important to the change process: past behavior, present behavior, and intention towards future behavior. Furthermore it is a powerful heuristic in organizing the other constructs of the Transtheoretical Model.

The Stage of Change variable has received an extensive amount of support across many behaviors, including smoking (Prochaska \& DiClemente, 1983), sun exposure (Rossi, 1989), alcohol (DiClemente \& Hughes, 1990), psychological distress (Prochaska, Rossi, \& Wilcox, 1991), and cocaine use (Rosenbloom, 1991) among others. Stages can be conceptualized for both the cessation and acquisition of behaviors. Stage of Acquisition has been most extensively validated for the acquisition of health promoting behaviors such as regular exercise (Marcus, Rossi, Selby, Niaura, \& Abrams, 1992), and safe-sex practices (Prochaska, Redding, Harlow, Rossi, Velicer, in press). Studies of the acquisition of negative or unhealthy behaviors have been largely limited to the investigation of the acquisition of 
tobacco use in adolescents (Elder, DeMoor, Young, Wildey, Molgaard, Golbeck, Sallis, \& Stern, 1990; Stern, Prochaska, Velicer, \& Elder, 1987).

The most extensive research has been on smoking cessation. Results have shown that Stage of Change is a strong predictor of future smoking behavior, out-predicting traditional demographics variables (Prochaska et al., 1985). Smoking subjects in Preparation have twice the likelihood of having quit at a six month follow-up than Contemplators, who in turn have twice the likelihood of quitting as Precontemplators (DiClemente, Prochaska, Fairhurst, Velicer, Velasquez, \& Rossi, 1991).

The Stage of Change variable can also be used to characterize populations in relation to the behavior of interest. A population with a large number of Precontemplators tends to be static, and perhaps in need of education as to the hazards of their behavioral problems. A population with large numbers in Contemplation, Preparation, and Action is actively changing and probably experiencing high levels of relapse along with positive stage movement. A population with large numbers of Maintainers has already made significant behavioral change. This might be the result of some combination of significant effort taken (e.g. smoking) and/or ease of changing the behavior (e.g. acquisition of seat belt use).

Research to date on the Transtheoretical Model has shown that the nature of the change process varies across the stages (Prochaska \& DiClemente, 1983), suggesting that optimal helping interventions would be designed to match and foster change mechanisms that are stage appropriate. Empirical research to date has supported this contention (Prochaska, DiClemente, Velicer, \& Rossi, 1993). Furthermore this observation suggests a simple explanation for the low success and recruitment rates that many traditional behavior change interventions have, namely that Action oriented programs are inappropriate for populations with large numbers in Precontemplation or Contemplation stages. Because of these reasons, matching intervention to stage is one of the cornerstones of programs based on the Transtheoretical Model. 
The stage variable is also a useful outcome measure. Since the differences among the first three stages are intentional differences, early stage progress is not registered by most traditional behavioral outcome measures, yet it has been shown that stage is a strong predictor of future behavioral change, suggesting that positive stage movement alone might be considered a positive outcome, especially when using short term follow-up assessments.

Two methods of assessing Stage of Change have been developed. This study uses the algorithmic approach in which clear decision rules are applied to the answers to a few items which ask about past and present behavior and behavioral intention to place individuals into distinct stages. This method has been very successful in areas where there are clear criteria for the behavior and its successful cessation (e.g. smoking). This method has proven somewhat more difficult to operationalize in areas where the criteria for successful behavior change are not obvious to the subjects (e.g low fat diet), but has also proven predictive (Rossi, Rossi, Prochaska, and Velicer, 1992).

An alternative method is to use a multi-dimensional instrument that measures agreement with attitudes characterizing each of the stages of Precontemplation, Contemplation, Action, and Maintenance (see Study II). This instrument, called the University of Rhode Island Change Assessment (URICA), was initially developed as a generic instrument asking the subjects about their "problem", and was applied to psychotherapy clients (McConnaughy, DiClemente, Prochaska, \& Velicer, 1989; McConnaughy, Prochaska, \& Velicer, 1982). Others have constructed, problem specific scales to measure these attitudes (Rollnick, Heather, Gold, \& Hall, 1992). The scale method is more complex than the algorithmic, and often does not stage all subjects into clearly identifiable groups, but has the advantage of providing a greater amount of information about subjects.

\section{RESEARCH ON STAGE OF CHANGE FOR ALCOHOL USE}

There have been a number of studies to date that have investigated the Stages of Change for alcohol use in various populations. DiClemente and Hughes (1990) used the 
URICA with a population of out-patient alcoholic adults. The four scales had internal consistency coefficients of between .69 and .82 . Cluster analysis found five distinct groups, three of which closely matched the stages of Precontemplation, Contemplation, and Action. As expected in this population of drinking or very recently sober alcoholics, a Maintenance group was not found. The other two groups were characterized as Ambivalent and Uninvolved. The first of these groups can be thought of as between Precontemplation and Contemplation, and the second showed evidence of feeling defeated in their attempts to stop drinking. What is of interest about this study was the finding that less than $50 \%$ of the subjects were in the Contemplation or the Participation (Action like) sub-groups despite seeking out treatment.

Snow's research (1991) investigated a population at the other end of the change process from the above study. His study of sober alcoholics was mainly an investigation of the processes of change of the Transtheoretical Model, but he found that the Action, Maintenance, and Termination stages were meaningful groups. This study is one of the few studies to empirically investigate the Termination stage. Snow investigated this stage using two definitions: 1) having greater than five years sobriety, and 2) having full confidence in their ability to stay sober and no temptation to drink over a variety of situations. These two definitions had substantial if not full agreement. Both of these studies investigated populations for which abstinence from alcohol was the stated goal.

There have also been a number of studies examining alcohol consumption within a moderation rather than an abstinence paradigm. One study reported the results of a random phone survey that staged respondents for three drinking behaviors: averaging three or more drinks per occasion, occasionally drinking six or more drinks, and driving after drinking three or more drinks in the last hour (LaForge, Rossi, \& Migneault, 1994). It was found that this population was much further along the stages for the behavior of drinking and driving than for their average or occasional drinking patterns. 
Rollnick et al (1992) also investigated stages of change, using a URICA type scale instrument. They developed a three scale instrument specific to alcohol use using a population of heavy drinkers (a Maintenance scale was not applicable). They felt that the scale method was especially appropriate in an area with ambiguous criteria. They presented substantial data attesting to the validity of the scales, but unfortunately did not perform cluster analysis to fully investigate sub-groups within the population.

In a study conducted by this author (Migneault, 1992) the stage construct was investigated in a college population. In this study the algorithmic method was used to stage subjects into stages relative to gender specific levels of alcohol use that represented long-term health risk. These levels entailed consuming more than two drinks for women or more than three drinks for men during average drinking occasions. For both acquisition and cessation, the four originally conceptualized stages were assessed (Precontemplation, Contemplation, Action, and Maintenance). No students were found in the Contemplation for Acquisition stage, and only $2.4 \%$ of the sample was in Action for Acquisition. About half of the sample was in Precontemplation for Cessation and $18 \%$ was in Precontemplation for Acquisition. No other stage had more than $10 \%$ of the sample and $10 \%$ was not staged because of contradictory or missing item responses.

The distribution across genders was almost identical. Differences in stage distribution between the under and upper classes across the four stages of cessation approached significance $(p<.06)$. The upperclassmen were approximately twice as likely to be in the cessation stages of Contemplation, Action, and Maintenance as underclassmen.

The validity of these stages was investigated by examining the relationship of stage to 11 dependent variables hypothesized to be related to alcohol use and abuse. The stage variable explained between 42 and $49 \%$ of the variance of the dependent variable set. It was found that there were significant differences on eight of these variables, and that the patterns of differences generally supported the validity of the stages as conceptualized. 
Despite this research showing strong support for the Stages of Change as applied to a college population there were a number of problems and areas of possible improvement in the staging procedure used. First, the drinking patterns of this population are quite unstable, making the justification of using a criterion based on long-term risk suspect. Second, the serious negative consequences most directly linked to typical consumption patterns for college students are acute problems. Basing research on levels of drinking implicated in the development of chronic diseases is not fully appropriate for a population that has unstable drinking habits. Thirdly, the typical college student drinks widely varying amounts across days of the week, making the concept of average amount consumed less meaningful (Baer, Stacy, \& Larimer, 1991). Practical concerns included the fact that the items were unwieldy and led to a significant proportion of the subjects not being staged.

\section{Stage Investigation Design FOR THE PRESENT StUdy}

In this study the construct of Stage of Change for immoderate drinking was investigated by using a short item set in a discrete algorithm with a college student population. This population was chosen because of the extent of immoderate drinking and the significant level of change in drinking patterns that occurs in college.

Building on what was learned in the previous study, this study will use an algorithm that stages all respondents into a stage of acquisition or cessation, that is based on criteria defined by risk of acute consequences of alcohol consumption, is gender specific, and accounts for the variable drinking habits that characterize a college population.

There is a difficulty in picking criteria for a level of drinking that does not entail significant risk of short-term negative sequelae. Even within genders there is a high degree of variability between individuals as to their sensitivity to alcohol, depending on variables such as weight, genetic make-up, drinking history, and personality characteristics.

To investigate this issue a preliminary study was conducted. A survey instrument was administered to 223 students in Introductory Psychology. One section pilot tested six sets of 
staging items each of which was designed to stage respondents into one of ten stages of acquisition or cessation. These item sets used different alcohol consumption criteria which varied in quantity and regularity of drinking. Three sets used the criterion of usually drinking more than 4,5 , or 6 drinks at least once in a typical week, the other three sets used the criterion of occasionally drinking more than 5, 6, or 7 drinks (see Appendix A). Reasonable stage distributions were found for each criterion with the proportion of the sample in Precontemplation for Acquisition increasing and the proportion in Precontemplation for Cessation decreasing with increasing drinking criteria. For all of the staging algorithms there were no appreciable numbers of respondents in the Contemplation or Preparation stages of Acquisition.

Because of constraints on survey length only two staging item sets were included in the final questionnaire. The criterion used in one was whether in a usual week subjects typically drank four or more drinks and in the other, five or more drinks on at least one occasion. This item stem was chosen because it was felt that this would more precisely assess the modal pattern of regular weekend heavy drinking typical of college students than either the simpler "average amount drunk" or vague "occasionally drink". Although all subjects were asked both staging item sets, the five or more set was targeted at men and was chosen because this is a widely accepted definition of heavy drinking (see Johnston, O'Malley, \& Bachman, 1992). The lower criterion (four or more) was chosen as being appropriate for women to adjust for their greater sensitivity to alcohol. Staging distributions for both genders using both criteria are reported.

A set of variables external to the Transtheoretical model was examined to investigate the external validity of the Stages of Change and to help characterize them. Most importantly, valid Stages of Change should show an interpretable relationship to consumption variables and to negative sequelae of drinking. To assess the negative consequences of drinking, a measure of alcohol-related experiences was developed in this study. Means by Stage for both consumption variables and the alcohol-related experience scales should increase 
with progression through the Stages of Acquisition and decrease with progression through the Stages of Cessation.

The relationship of Stage of Change to a set of measures of psychosocial development was also assessed. The modal pattern of changes in alcohol consumption through adolescence and young adulthood is to progress from non-drinking to excessive drinking and then to reduce alcohol consumption to light or moderate levels (Harford, 1984; Johnston et al., 1992). This temporal sequence can also be conceptualized as the acquisition and cessation of immoderate drinking. This period of time is also one of psychosocial development which includes the separation from parents and family, growing importance of peer relationships, and a self-definition as an adult within society. The fact that for many individuals psychosocial development and the acquisition and eventual cessation of immoderate drinking are concurrent processes suggest the possibility of a relationship between these two progressions. In an exploratory attempt to assess this relationship, subjects were administered measures of psychosocial development and the empirical relationship to Stage of Change was examined. It was hypothesized that a positive relationship between stage progression and measures of psychosocial development would be observed. 


\section{METHOD}

\section{SUBJECTS}

Subjects were 629 students surveyed at the University of Rhode Island in the fall of 1993 and spring of 1994 between the ages of 18 and 25 years and 11 months. The majority of the subjects were recruited from undergraduate classes in the psychology department, although a variety of other classes were also sampled including physical education, pharmacology, nutrition, and sociology. A small percentage were recruited from the fraternities and the university health center.

The sample was $66.3 \%$ female, and $94 \%$ white. The distribution across the classes was $17.4 \%$ freshmen, $32.2 \%$ sophomores, $19.1 \%$ juniors, $26.2 \%$ seniors, and $5.3 \%$ either fifth-year or non-matriculated. The average age was 21.3 years. Of these students $47 \%$ were classified as in-state and $39 \%$ lived in dormitories, $10 \%$ lived in fraternities or sororities, and $51 \%$ lived off campus.

The use of alcohol in this sample was substantial. Using a criterion of at least one drinking occasion in the last 30 days, $92 \%$ were classified as active drinkers. These subjects drank on average 8.6 days a month and consumed on average 5.3 drinks per occasion. There were substantial differences in drinking by gender with drinking women averaging 7,3 days per month and 4.4 drinks per occasion. For men these figures were 11.0 days and 6.9 drinks. Drinking women reported an average maximum amount drunk on any occasion in the past month as 6.8 , and men as 12.1 . Distributions by gender for both number of drinking days in the last 30 and for average drinks per occasion are presented in Figure 2-1 and Figure $2-2$, respectively.

\section{INSTRUMENTS}

The survey administered contained 282 questions, of which four item groups are of specific interest to this study. The full survey instrument is presented in Appendix A. 
Demographic Assessment

A set of 22 items asked about basic demographic information and drinking history. Variables examined in this study include age, gender, college class living situation, number of days in the last month that alcohol was consumed (Days), the number of drinks consumed during a typical drinking occasion (Drinks), and the number of drinks consumed before subjects start to feel intoxicated (Intoxication).

\section{Stages of Change: Algorithmic Assessment}

The staging algorithm used answers from a branched set of five items. The first item asked if the subject in a typical week usually had four or more drinks on one or more occasions. If the subject answered 'Yes' they were asked two questions about the length of time they had been doing so and their intention to reduce their drinking to less than this amount. If they answered 'No' they were asked whether they intended to start drinking at this level in the next six months or in the next 30 days and, if they drank at or above this level in the past, how long ago had they stopped. A second identical set of items asked these same questions using the criterion of five or more drinks.

For each criterion subjects were classified into one of nine groups that represented the five Stages of Acquisition and the five Stages of Cessation with one group representing both the Maintenance stage of acquisition and the Precontemplation stage of cessation. The algorithm used is presented in Table 2-1. The traditional six month criterion for Maintenance was used. Most response patterns clearly determined the subject's stage, but because both acquisition and cessation stages were assessed some decision rules had to be formulated to assign some subjects to a stage. Specifically some students who met the criteria for Action for Acquisition, also indicated an intention to reduce their drinking in the next six months or next 30 days. It was decided that the Stages of Cessation would be given priority, and these subjects would be classified into either Contemplation or Preparation as appropriate. This decision was made on the strength of previous research on the Stages of Cessation. 
Furthermore, it seems reasonable that subjects have acquired the behavior if they feel that they must delay a reduction until they are ready.

Similarly, a few subjects who were in the stages of Action or Maintenance for Cessation also expressed an intention to resume heavier drinking in the next six months or the next 30 days. Again priority was given to the cessation stage on the strength of previous research. Furthermore, the item that assesses this intention would not have been asked if only Stages of Cessation were being assessed, and it was thought that this question might have been somewhat confusing to these subjects.

\section{Alcohol-Related Experiences}

A set of 31 items that assessed alcohol-related experiences was created. This item set included items that ask about a wide range of acute negative results of excessive drinking including physical effects (e.g. been sick, been hungover), emotional effects (e.g. felt guilty, had a personality change), interpersonal effects (e.g. criticized by a date, gotten into an argument or fight), and effects on school/vocational activities (e.g. had job problems, missed class). Also included were behaviors that entail significant risks (e.g. driven after drinking, had unprotected sex), and items that are recognized signs of problematic involvement with drinking (e.g. unable to stop thinking about alcohol, felt guilty about your drinking).

Items came from a number of sources. Some were taken from previous research efforts on adults in general (Babor, Kranzler, \& Lauerman, 1987; Fillmore \& Midanik, 1984) or on adolescents or college students in particular (Berkowitz \& Perkins, 1986; Engs, 1977; White \& Labouvie, 1989). In addition, two diagnostic instruments were modified and included. The first is the CAGE instrument, which is a 4-item instrument named after a key word in each item (Cutdown, Angry, Guilty, and Eye-opener; items 16, 17, 10, and 15 respectively). This instrument is used to diagnose problem or alcoholic drinking (Mayfield, McLeod, \& Hall, 1974). The second instrument from which items were taken was the AUDIT, a cross-culturally valid World Health Organization instrument designed to detect 
hazardous drinking, which is conceptualized as a type of alcohol involvement that will lead to significant problems (Saunders \& Aasland, 1987). The seven-item short form of this instrument was used (items $4,8,10,11,13,15$, and 25). Item stems and response formats for both of these instruments were modified so a consistent item presentation could be maintained. In addition, two items that assess emotional sequelae of drinking were created in an exploratory attempt to assess under-investigated problems that may be more commonly experienced by women (items 26,27 ).

Subjects were asked to circle the number of times in the last 12 months that they had had each experience related to their alcohol consumption. The following 6-point response scale was provided: $0,1,2,3-5,6-9,10$ or more times.

\section{Measures of Psychosocial Development}

Three scales measuring aspects of psychosocial development were included in a 32item set of questions. These scales are subscales of the Student Development Task and Lifestyle Inventory (SDTLI), which has received intensive development over the last 15 years (Winston \& Miller, 1987). The scales were based on the work of Chickering (1969).

The first subscale, Peer Relationships, measures the subject's ability to develop relationships that have greater openness, trust, and independence, to resist pressure to conform, and to accept differences. The second scale, Emotional Autonomy, measures the ability of subjects to be free from the need for continuous reassurance, to be prudent in risk taking, to have confidence in their decision making, and to voice dissenting opinions. The third scale, Lifestyle Planning, measures the establishment of a personal direction in one's life that incorporates values, family plans, and vocational objectives. Subjects who score high on this subscale have the capacity to follow through on commitments and can specify how currents activities relate to their goals.

In an attempt to improve the reliability of these scales the item format was changed from a True/False to a 5-point Likert scale varying from $l=$ Strongly disagree to $5=$ 
Strongly agree. This response format is generally deemed to produce superior psychometric properties (Comrey, 1988).

\section{PROCEDURE}

The survey for this study was included within a larger survey that assessed additional aspects of drinking behaviors and attitudes. Informed consent was obtained and subjects were assured of the confidentiality of their responses (See Appendix B for a copy of the consent form). Surveys were completed during class time, or subjects took the survey home and returned it. Most, although not all, of those doing the survey on their own time received a small amount of class credit for returning the survey. A few students who completed the survey at the health services center were given a pen emblazoned with the university name in exchange for completing the survey. 


\section{RESULTS}

\section{COMPARISON OF STAGING ALGORITHMS}

Using the algorithm presented in Table 2-1 each subject was staged into one of nine groups for both the lower drinking criterion of typically drinking four or more drinks at least once in a usual week and the higher criterion of five or more drinks. For each criterion less than $3 \%$ of the subjects were unstaged due to missing data. Less than $1 \%$ of subjects were classified into Contemplation for Acquisition for both criteria $(\underline{\mathbf{N}}=2$ and $\underline{\mathbf{N}}=4)$. Cell size this small renders analysis unreliable and this stage will not be further investigated. No subjects were staged into Preparation for Acquisition for either criterion. Distributions are presented in Table 2-2.

Distributions across genders differed for both criteria (4 or more drinks: $\chi^{2}(6)=26.54, \mathrm{p}<.0001 ; 5$ or more drinks: $\left.\chi^{2}(6)=43.69, \mathrm{p}<.0001\right)$. In general females are over-represented in stages entailing drinking below the criterion (Precontemplation for Acquisition and Action and Maintenance for Cessation) and under-represented in Precontemplation and Contemplation for Cessation. These distributions are also presented in Table 2-2.

Comparison of stage distributions across criteria was done separately for each gender. Kappa was used as a measure of agreement. For the female sample, stage distributions using the lower and the higher criteria had a kappa of .69 , and for men, .78 . These numbers represent high agreement. Subjects' cross-classification frequencies by gender are presented in Table 2-3.

Of the 603 subjects with complete staging data, 130 subjects $(22 \%)$ were staged differently using the two criteria. Of these about half $(\underline{N}=63,10 \%$ of the total sample) had acquired the behavior of drinking 4 or more drinks at least once a week but had not fully done so for 5 or more drinks (in the acquisition stages of Precontemplation or Action). A similar number $(\underline{\hat{N}}=57,9.5 \%)$ were in a more advanced stage of cessation for the higher 
criterion level than the lower. For example, there were 13 subjects who were in Contemplation for Cessation for the higher criterion while in Precontemplation for Cessation for the lower. A few subjects $(\mathrm{N}=7,1.2 \%)$ had contradictory staging across the two criteria. For example one subject was classified in the cessation stage of Maintenance for the lower criterion and Action for the higher criterion.

The pattern of stage cross-classification by criteria was very similar for both genders. Overall the major difference between the genders was that a somewhat higher percentage of women changed stage with the different criteria than men ( $25 \%$ vs $19 \%$ ) suggesting that the difference between the criteria is more significant for women. Also a higher percentage of men had contradictory staging than women $(2.0 \%$ vs $.8 \%)$.

A final stage classification was accomplished by using the lower criterion for women and the higher one for men and is presented in Table 2-4. Using these gender specific criteria reduces distribution difference between the genders compared to using the same criterion for all subjects. Nevertheless these differences remain significant $\left(\chi^{2}(6)=15.37, \mathrm{p}<.05\right)$, The pattern of differences is similar to those found for each criterion as described above (women over-represented in light drinking stages) with the exception that women are proportionally over-represented in the Action for Acquisition stage as compared to men (6.7\% of women vs $3.9 \%$ of men).

As acquisition and cessation are conceptualized as independent processes, further analysis will be conducted for the stages of acquisition and the stages of cessation separately. This entails conceptualizing one group of subjects as both the Maintenance for Acquisition stage and the Precontemplation for Cessation stage.

\section{EXTERNAL VALIDITY}

The relationship of the algorithmic stages to a set of variables presumed to be related to alcohol-related behavior was examined. Differences across the Stages of Change help characterize the stages and if consistent with theory, provide evidence of the validity of this 
way of measuring this construct and of the applicability of the Transtheoretical Model of Change to college drinking.

Validating variables used in this study can be conceptualized into four categories. The first, demographic variables include gender, class, living situation, and age. The second is alcohol consumption variables. These include number of drinking days in the last month (Days), number of drinks usually consumed when drinking (Drinks) and the number of drinks consumed when subject first starts to feel intoxicated (Intox). There were two other survey items similar to the intoxication item that asked about number of drinks it takes to start to feel the effects of alcohol and the number to be drunk. Analysis showed these three variables to be highly correlated and to possess a nearly identical relationship to stage of change. Furthermore combining all three as a scale did not appreciably add to the results. Therefore, only the results for the intoxication item are reported.

The third category contains three scales measuring types of alcohol-related experiences. The development of these three scales from a 31-item questionnaire is reported below. In the fourth category are three scales measuring elements of psychosocial development that were taken from the literature and were described in the methods section. In this sample, the coefficient alphas for these three scales were .68 for Peer Relations, .59 for Emotional Autonomy, and .76 for Lifestyle Planning.

The demographic variables were examined using chi-square analysis for categorical variables and analysis of variance for age. Each of the other three sets of variables were first entered into a MANOVA to control for family-wise error, and then if appropriate, follow-up ANOVA and Tukey tests were conducted. These analyses were conducted separately for the stages of acquisition and the stages of cessation. Before these analyses are reported the development of the Alcohol-Related Experiences instrument is presented. 


\section{Development of the Alcohol-Related Experiences instrument}

Exploratory component analysis of the item set was conducted using principal components analysis (PCA). Cases were deleted if they had more than $10 \%$ missing data across the 31 items. Also subjects who answered "0" to all 31 questions were deleted. Although these answers are valid, they do not assist in the investigation of the underlying structure of item set of alcohol-related problem and will inflate the inter-item correlations (Jackson, 1970, Velicer, DiClemente, Rossi, \& Prochaska, 1990). The final sample consisted of 569 subjects or $90.5 \%$ of the original sample.

A PCA was conducted on the 31 by 31 matrix of intercorrelations created using pairwise deletion. The number of components to retain was determined by comparing the results of the scree procedure (Cattell, 1966), minimum average partial procedure (Velicer, 1976), and parallel analysis (Horn, 1968; Lautenschlager, 1989). The scree procedures suggested retaining four components and the other two procedures indicated three as being the correct number of components to retain. Both the three and four component solutions were evaluated. The four component solution included a component which, although interpretable as representing risk taking, was composed of only three high-loading items and explained only $3.7 \%$ of the total variance. Furthermore, Monte Carlo studies have indicated that the minimum average partial and parallel analysis are more accurate indicators of the number of components to retain (Zwick \& Velicer, 1986). This fourth component could be the basis of further scale development as these results suggest that the generation of additional items might result in a well defined component measuring alcohol related risk taking. The three component solution was selected for further interpretation.

Both varimax and oblique rotations were performed. The oblique rotation was chosen as more interpretable. Inter-component correlations were moderate and varied from .37 to .43. Of the 31 items, eight were deleted because of complex or low loadings, and a final PCA was performed on the remaining 23 items. The first component was interpreted as measuring the problems associated with excessive drinking and was labeled Excess, was 
composed of ten items, and had a coefficient alpha of .90 . The second component was composed of seven items, was interpreted as measuring internal distress caused by drinking and was labeled Distress. It had a coefficient alpha of .85. The third component was composed of six items and was interpreted as measuring consequences of alcohol consumption that are associated with established problem drinking. It had an alpha of .80 and was labeled Problem. Together these three scales explained $51.4 \%$ of the variance in the reduced item set. Items and their component loadings are presented in Table 2-5.

Scale scores were completed by taking the mean of items responses for each scale. Cases with more than two items missing were deleted. Scale means, standard deviations, coefficient alphas, and interscale correlations are presented in Table 2-6.

The relation of these scales to three other variables was assessed to provide some construct validity evidence. First, correlations between the scales and two consumption variables, Days and Drinks, were calculated. Results show that the Excess scale is highly correlated with the consumption variables (Days: $r=.64$; Drinks: $r=.62$ ), supporting its interpretation as being related to excessive drinking. The other two scales had moderate correlations with both variables ranging from .36 to .42 . Also, t-tests were conducted to assess the effect of gender on scale scores. It was found that there was a significant effect of gender on the Excess and Problem scales, with men scoring significantly higher on the former two scales. The t-test on the Distress scale was not significant. This supports the hypothesis that women are less likely to 'act out' when drinking, but rather experience psychological effects as do men.

\section{Stages of Acquisition}

The staging algorithm used in this study classified significant numbers of subjects into only three of the five possible Stages of Acquisition: Precontemplation, Action, and Maintenance. These stages were investigated by examining difference across stages on 
demographic variables, variables related to alcohol consumption, and a set of developmental variables hypothesized to be related to the stage variable.

Demographics. Analysis using the chi square statistic was conducted on the relationship between stage and gender, college class, and living situation. Distributions of the Stages of Acquisition in total and across these variables are presented in Table 2-7.

Results show that Stage of Acquisition and gender were significantly related $\left(\chi^{2}(2)=9.27, \mathrm{p}<.05\right)$. Women were over-represented in the Precontemplation and Action stages while a much higher percentage of men were in Maintenance, having fully acquired this behavior. This result suggests that a greater proportion of men have fully acquired the habit of immoderate drinking, while the women in this college-aged sample are nearly twice as likely to be in the process of acquiring the habit (in Action).

Stage of Acquisition and living situation were also highly related $\left(\chi^{2}(6)=51.35\right.$, $\underline{p}<.001)$. Because of the small number of subjects who own their own home $(\underline{n}=4)$ this category was not included in this analysis. Comparing living situations in pairs showed that subjects living in dormitories and with their parents were similar as were those living in fraternities or sororities and those living off-campus. Students living in the first two situations were less progressed along the Stages of Acquisition with higher percentages in Precontemplation and lower percentages in Maintenance. Those living in dormitories were also over-represented in the Action stage suggesting that this is the living situation where the most new acquisition occurs. An examination of differences between residents of sororities and fratemities showed no significant differences, but this finding might be a result of the small number of subjects in these groups.

Stage distribution was also related to class $\left(\chi^{2}(6)=33.37, \mathrm{p}<.001\right)$. The few fifth year or non-matriculated students in the sample were not included in this analysis. All pairwise comparisons of class were significant except between sophomores and juniors and between juniors and seniors. In general there was a tendency for progression through the 
stages of acquisition as grade level increased. For example, freshman were over-represented in Precontemplation and Action and highly under-represented in Maintenance, while seniors were highly under-represented in Precontemplation and highly over-represented in Maintenance.

Differences between the mean age of subjects across the Stages of Acquisition were investigated and proved to be significant $\left(\underline{F}(2,403)=6.38, \underline{\mathrm{p}}<.01, \omega^{2}=.03\right)$. Follow-up Tukey tests showed that students in the Action stage were significantly younger than either the Precontemplation or Maintenance stages. This result suggests both that most new acquisition of immoderate drinking occurs early in college, which coincides with the finding that $53 \%$ of student in Action for Acquisition are freshman. The older age of Precontemplators suggests that progression is not just a developmental process, but that some students never progress to immoderate drinking. See Figure 2-3 for a graphic representation of stage means by age. See Table 2-8 for means and standard deviations by Stage of Acquisition for age and all other continuous variables reported in this section.

Alcohol consumption. The three alcohol consumption variables, Days, Drinks, and Intox were entered into a MANOVA which proved significant $(\Lambda(6,734)=.55, \underline{\mathrm{p}}<.0001)$ accounting for $45 \%$ of the variance. Follow-up ANOVAs were all significant (Days: $\left(\underline{\mathrm{F}}(2,405)=135.25, \mathrm{p}<.0001, \omega^{2}=.40\right)$; Drinks: $\left(\underline{\mathrm{F}}(2,405)=128.52, \underline{\mathrm{p}}<.0001, \omega^{2}=.39\right)$; Intox: $\left(\mathrm{F}(2,372)=39.00, \mathrm{Q}<.0001, \omega^{2}=.16\right)$. Follow-up Tukey tests showed that with progression from Precontemplation to Action and from Action to Maintenance there is a significant increase for all three variables. The patterns of means are presented in Figure 2-4.

The increase in number of drinks with progression through the Stages of Acquisition is clearly expected since the stage definition is dependent on amount drunk. The steeper increase in number of drinking days is less dependent on stage definition, with those in Maintenance drinking on average three times more often than the stage definition requires. 
The combination of changes in these two variables probably explains the linear increase in tolerance across the stage as evidenced by the variable Intox.

Alcohol-Related Experiences. The relationship between the Stages of Acquisition and the Alcohol-Related Experiences scales was also examined using analysis of variance techniques. The MANOVA was significant $(\Lambda(6,794)=.48, \mathrm{p}<.0001)$ accounting for $52 \%$ of the variance. Follow-up ANOVA's, and pair-wise Tukey tests were conducted. Results for the Excess and the Distress scale were similar. Both ANOVA's were significant (Excess: $\left(\underline{\mathrm{F}}(2,399)=214.63, \mathrm{~g}<.0001, \omega^{2}=.52\right)$; Distress: $\left.\left(\underline{\mathrm{F}}(2,399)=56.19, \mathrm{q}<.0001, \omega^{2}=.22\right)\right)$ and Tukey tests suggest that each stage is significantly different from the other stages, with increasing scale scores with stage progression. Analysis of the Problem scale was also significant $\left(\underline{\mathrm{F}}(2,399)=25.38, \mathrm{~g}<.0001, \omega^{2}=.11\right)$ and follow-up Tukey tests showed that subjects in Maintenance scored significantly higher on this scale than did those in Precontemplation or Action. Scores for all three scales were converted to T-scores $\underline{M}=50$, $\underline{S D}=10$ ) and are presented by stage in Figure 2-5.

These results of the analysis of the Alcohol-Related Experience scales suggest that those who are further along in acquisition experience more negative sequelae due to their alcohol consumption, supporting the contention that stage membership has meaningful consequences. Furthermore the significant increase in the Problem scale only occurring between the Action and Maintenance stages is consistent with the conception of this scale as measuring experiences secondary to more severe chronic drinking problems.

Psychosocial development scales. The MANOVA investigating the three developmental scales was significant $(\Lambda(6,792)=.94, \underline{\mathrm{p}}<.001)$ but only accounted for $6 \%$ of the variance. Follow-up one-way ANOVA's and Tukey tests were performed. Analysis of the Peer Relationship scale showed no significant differences by stage. The differences across the Stages of Acquisition for the Emotional Autonomy scale approached significance 
$\left.\underline{F(2,399)}=2.90, p=.056, \omega^{2}=.01\right)$, with follow-up Tukey tests suggesting that

Precontemplators score higher on this scale than those in Action. Differences on the Lifestyle Planning scale were significant across Stage $\left(\mathrm{F}(2,399)=8.96, \underline{\mathrm{g}}<.001, \omega^{2}=.04\right)$. Based on

Tukey tests subjects in the Precontemplation stage scored higher than the subjects in either the Action or Maintenance stages. T-scores by Stage for all three developmental scales are present in Figure 2-6.

Overall the relationship observed between Stages of Acquisition and the three measures of psychosocial development was weak and inconsistent. Certainly there was no evidence for the hypothesis of increasing psychosocial development with stage progression for these Stages of Acquisition. The flat pattern of Peer Relationship means across the Stages is surprising considering the strong relationship supported in the research literature between heavy drinking and peer influences (Baer et al,, 1991; Jessor, 1987). The low internal reliability for this scale might have contributed to this finding. The pattern of means of the Emotional Autonomy scale across the Stages of Acquisition parallels that of age across these Stages and is probably an artifact of this relationship. The relationship between Lifestyle Planning and Stage suggests a positive relationship between the decision not to drink immoderately in a heavy drinking environment and increased planning of ones future, but the effect size of this finding was small.

\section{Stages of Cessation}

The staging algorithm used in this study classified subjects into five Stages of Cessation: Precontemplation, Contemplation, Preparation, Action, and Maintenance. These stages were investigated by examining difference on the same set of demographic, alcoholrelated, and developmental variables that were investigated for the Stages of Acquisition. Stage of Cessation distributions both in total and across the categorical variables of gender, living situations, and class are presented in Table 2-9. 
Demographic variables. $\quad$ Results show that Stage of Cessation and gender were significantly related $\left(\chi^{2}(4)=10.09, \underline{\mathrm{p}}<.05\right)$. Women were under-represented in Precontemplation and over-represented in Action and Maintenance, suggesting that a higher proportion of women who had had a period of immoderate drinking had reduced their drinking to moderate levels.

Stage of Cessation was not significantly related to either living situation or class. As a follow-up, students were separated into underclassmen (freshmen and sophomores) and upperclassmen (juniors and seniors). Chi square analysis reveals a significant association $\left(\chi^{2}(4)=10.29, \mathrm{p}<.05\right)$. The underclassmen were over-represented in Precontemplation and Preparation and under-represented in Contemplation and Maintenance. A context for understanding this complicated pattern of results can be created by initially combining similar stages. Combining the Contemplation and Preparation stages shows no differences between under and upperclassmen, suggesting that both groups have a similar proportion considering changing their drinking habits. Understanding why more underclassmen are planning more immediate change awaits further research. Although in both groups most subjects are presently drinking immoderately (combining the first three stages), this proportion is lower in upperclassmen, suggesting a net stage progression over time in college.

A significant relationship between Stage of Cessation and subject age was found $\left(\underline{F}(4,419)=9.92, \underline{\mathrm{p}}<.0001, \omega^{2}=.08\right)$, and follow-up Tukey tests showed that students in the Maintenance Stage were older than those in the other four stages. The age to Stage relationship is presented graphically in Figure 2-7. (See Table 2-10 for means and standard deviations by Stage of Cessation for all continuous variables reported in this section.) The lack of a clear linear relationship between the earlier Stages and age, despite the significantly older age of Maintainers suggests that younger students are making change attempts (preparing and acting) but are experiencing high rates of relapse. The demonstration of this hypothesis awaits retrospective or longitudinal research. 
Alcohol consumption variables. The Stages of Cessation showed a strong relationship to the alcohol consumption variables. The result of the MANOVA was $(\Lambda(12,1095.6)=.64$ $\mathrm{p}<.0001$ ) indicating that the stage variable accounts for $36 \%$ of the variance. All three follow-up ANOVA's were significant (Days: $\left(\underline{\mathbb{F}}(4,420)=40.67, \underline{\underline{p}}<.0001, \omega^{2}=.27\right.$ ); Drinks: $\left(\underline{\mathrm{F}}(4,419)=30.87, \underline{\mathrm{p}}<.0001, \omega^{2}=.22\right) ;$ Intox $\left.:\left(\underline{\mathrm{F}}(4,418)=11.82, \mathrm{p}<.0001, \omega^{2}=.17\right)\right)$. Followup tests on the variable Days showed that subjects in Precontemplation, Contemplation, and Preparation drank on significantly more days in the last month than those in Action and Maintenance. Follow-up Tukey tests for the variable Drinks showed that Precontemplators and Contemplators consume more alcohol on average than those in Action or Maintenance, and that those in Preparation and Action consume more than those in Maintenance. Tukey tests on the variable Intox showed that subjects in Precontemplation started to become intoxicated at higher levels than those in Action or Maintenance, as did those in Contemplation and Preparation when compared to Maintenance. Means by Stage are presented in Figure 2-8. As can be seen in this figure there is an approximate linear relation between Stage of Cessation and these variables. As would be expected for these consumption related variables, the largest change occurs between the Preparation and Action Stages, the behavioral transition in stage progression.

Alcohol-Related Experiences. The relationship between Stage of Cessation and the three alcohol-related experiences scales proved to be significant $(\Lambda(12,1087.7)=.72, \mathrm{p}<.0001)$ accounting for $28 \%$ of the variance. All three follow-up ANOVA's were significant (Excess $\left(\underline{\mathrm{F}}(4,413)=35.55, \underline{\mathrm{p}}<.0001, \omega^{2}=.25\right) ;$ Distress: $\left(\underline{\mathrm{F}}(4,413)=13.09, \mathrm{p}<.0001, \omega^{2}=.11\right) ;$ Problem: $\left.\left(\underline{\mathrm{F}}(4,413)=6.88, \mathrm{Q}<.0001, \omega^{2}=.06\right)\right)$. T-scores by Stage are presented graphically in Figure 2-9. Follow-up Tukey tests showed that subjects in the Precontemplation, Contemplation, and Preparation stages reported significantly higher scores on the Excess scale than those in the Action and Maintenance Stages. Tukey tests also revealed that Precontemplators and Contemplators report significantly higher scores on the Problem scale 
than do those in Maintenance. Both of these scales exhibit an approximate linear relation to Stage, especially between Contemplation and Maintenance. A somewhat different pattern is observed for the Distress scale. Tukey tests showed that those in Precontemplation, Contemplation, and Preparation had higher frequency of distress due to their drinking than those in Maintenance, as did those in Preparation when compared to subjects in Action, but of additional interest is the peak at Preparation. Although non-significant, this is suggestive that students' motivation to stop drinking immoderately is related to felt distress about their drinking. This is similar to the relation between the Cons of a behavior and Stage of Change found in other behavioral domains (Prochaska, Velicer, Rossi, Goldstein, Marcus, Rakowski et al., 1994).

Psychosocial development scales. The MANOVA conducted on the three developmental scales was also significant $(\Lambda(12,1082.4)=.94, p<.05)$, but explained only $6 \%$ of the variance. Follow-up ANOVA's were not significant for either the Peer Relationship or Emotional Autonomy scales but was for the Life Planning scale $(\underline{F}(4,411)=3.14, \underline{\mathrm{g}}<.05$, $\omega^{2}=.02$ ). Followaup Tukey tests only suggest that Maintainers have a more developed life plan than Contemplators. T-score means by Stage are presented in Figure 2-10. This significant result might be explained by the older age and greater likelihood that Maintainers are seniors who must plan for their future. More important is the overall lack of either significant results or clear trends, not supporting the hypothesized positive relationship between Stage and psychosocial development. 


\section{DISCUSSION}

These results support the validity of the Stages of Change for college students as determined by the algorithm used in this study. The results will be discussed first with focus on the criteria chosen, then the stage distributions observed, and finally the external validity evidence investigated.

\section{CRITERIA FOR STAGING FOR IMMODERATE ALCOHOL USE}

The staging algorithm was designed to assess the behavioral and intentional relationship of subjects to a pattern of drinking that entails significantly increased risk of suffering negative experiences in a short to moderate time period. This pattern of drinking is defined by two characteristics: frequency of drinking and the quantity drunk. The frequency chosen for the stage criterion was at least once a week for most weeks. This was chosen as it reflects the typical college drinking pattern of drinking heavily on at least one weekend day (Baer et al., 1991). It was felt that assessing the more common 'average amount drunk' would underestimate the risk incurred by many students. It could be argued that less frequent excessive drinking also presents significant risk, such as once or twice a month, but for research purposes these distinctions are probably relatively inconsequential, with only a few subjects changing stage with small adjustments to the frequency aspect of the criterion.

The more important decision was the quantity to use for the criteria. A gender specific set of criteria was chosen to partially offset the differences between genders in the metabolism of ethanol. Using gender specific criteria produced a more similar stage distribution although the gender differences remained significant.

Less clear than the relation between gender and blood alcohol level (BAL) is the relation of BAL to the incurred risk of the negative sequelae most often experienced by college students and how this relationship is modified by gender. The results do show that the criteria used in the present study separated subjects into two groups with very different 
frequencies of negative consequences, arguably the most important outcome variable. Those who are above the criterion score from 2.7 to 4.4 times higher on the Alcohol-Related Experiences scales than those below criteria. These large group differences suggest that the criteria used are effective for defining groups.

Although using gender specific criteria is an improvement over non-specific criteria, the relationship between consumption and short term risk is altered by many other variables than gender. One promising development is the advent of computer-based expert system delivery modalities that could allow for more individualized determination of criteria by, in theory, taking into account variables such as weight, tolerance, family history, personality variables, and past history with alcohol related problems.

\section{STAGE DISTRIBUTIONS}

Since this sample is not representative, generalizations of the observed stage distributions to the college population must be tentative, although the large sample size increases the confidence that these results would not diverge greatly from population values. Overall the stage distributions obtained by this algorithm classified approximately one third of all subjects into Precontemplation for Cessation, one fourth into Precontemplation for Acquisition, and about one eighth into Maintenance for Cessation. No other stage had more than $10 \%$ of respondents. All told, $55 \%$ are drinking above criteria, which is consistent with the well documented heavy drinking occurring on American college campuses (Wechsler et al., 1994). Less than $2 \%$ of subjects were unclassified for either gender.

Compared to previous research on college alcohol use (Migneault, Stevenson, \& Velicer, 1994), the stage distribution found represents a smaller proportion in Precontemplation for Cessation ( $36.5 \%$ vs $57.1 \%$ ) and greater proportions in all other stages. This difference could be an artifact of sample differences, but is more likely to be a result of the present study using higher levels of consumption as stage criteria. With higher consumption levels there are more students who have never drunk at that level, fewer who are 
drinking at or above without ambivalence, and a greater proportion who have reduced their drinking to below the criteria.

\section{Stages of Cessation}

The main focus of this study was on the Stages of Cessation. The algorithm used was successful in staging substantial numbers of students into all the Stages of Cessation. Of those students who are or have been drinking above criteria, slightly over half are in Precontemplation, $14 \%$ are in Contemplation, 5\% are in Preparation, the smallest stage, $10 \%$ are in Action, and $20 \%$ are in Maintenance.

One interesting aspect of this distribution is that about $30 \%$ of those who have drunk immoderately are in an active Stage of Change, yet there are fairly low numbers of students in Maintenance. This suggests that students do not act on their intentions or if they do, do not successfully maintain their behavior change, which is consistent with longitudinal research showing that most successful cessation only happens after a cyclical pattern of stage transitions. This agrees with previous research for heavy drinking (Fillmore \& Midanik, 1984) and for adolescents and smoking (Pallonen, Murray, Schmid, Pirie \& Luepker, 1990). This high level of naturally occurring change or intention to change contradicts the common perception of college campuses as environments of intractable, unambivalent drinking (Baer et al., 1991). The failure of most intervention programs to reduce drinking is likely due, not to the fact that everyone is a Precontemplator, but to the fact that interventions typically do not take full advantage of naturally occurring change efforts, are action oriented, are often abstinence based, and do not help college students overcome the difficulty they have in maintaining reduced drinking.

\section{Stages of Acquisition}

The most important finding about the Stages of Acquisition is the failure of the algorithm to stage subjects into Contemplation or Preparation. The algorithm only separates 
subjects who have never drunk immoderately from those who have done so for either a short (Action) or longer (Maintenance) period of time. Since arguably, the main strength of the staging paradigm is finding meaningful distinctions between subjects who are similar on target behaviors, not finding these early stages is a serious weakness in this method of staging.

There are three possible reasons for this result. Either these distinct stages do not exist for this behavior and population, subjects pass through these stages too quickly and are therefore unlikely to be found in cross-sectional data, or the algorithm used was not effective in distinguishing the early Stages of Acquisition. The age profile of these stages does lend some support to the first option suggesting that all but a few young students have either acquired immoderate drinking already or will remain in Precontemplation for Acquisition.

Previous research does not provide clear evidence to chose among these alternatives. Most research on the Stages of Acquisition has largely focused on the acquisition of healthy behaviors such as exercise or safe sex, or on complicated combinations of acquisition and cessation such as moving to low-fat diets. Although this research has clearly validated the stages of Contemplation and Preparation, this change process is distinctly different from the acquisition of unhealthy behaviors.

Previous research into the acquisition of unhealthy behaviors includes acquisition of cigarette smoking in high school and previous research by this author into acquisition of alcohol use in adolescents and college students. For smoking, researchers have used both methods of staging subjects. Research with a URICA type instrument found only three scales, Precontemplation, Decision Making, and Maintenance, and five interpretable clusters (Stern et al., 1987). Two of the clusters were labeled Contemplation and Decision Making and included $4.4 \%$ and $15.8 \%$ of subjects respectively. The Decision Making cluster is hypothesized to be a cluster between Contemplation and Action, and entails some behavioral experimentation. Only a little validity evidence was presented, leaving these clusters largely unexplored. More recent research using an algorithmic method to stage vocational high 
school students on smoking acquisition found small numbers of students in Contemplation and Preparation (2.3\% and $3.5 \%$ of all students, respectively) (unpublished data).

Previous research into acquisition of drinking has used an algorithm with vocational high school students (Migneault, Pallonen, \& Velicer, 1994) and college students (Migneault, Stevenson, \& Velicer, 1994). The first of these found $1.7 \%$ of all respondents in Contemplation and Preparation of Acquisition combined. No appreciable numbers of college students were found in Contemplation (a separate Preparation stage was not assessed).

These results suggest that although a continuous measure might be able to identify larger Contemplation and Preparation stages for acquisition, the algorithmic method does not. This suggests a basic difference between this type of behavior change and other forms for which the algorithmic method is effective.

This difference can be explored theoretically. Acquisition of unhealthy behaviors usually involves the development of a habit that is reinforcing but is opposed by selfpreservative concetns, internal moral strictures, and/or external social influences. This alignment of influences is largely reversed for the cessation of unhealthy behaviors.

For example, for a smoker to positively endorse an item asking if they are planning to quit in six months is to express their agreement with the moral, safe, and societally approved position, which are the forces pushing behavior change. If questioned, these Contemplators are likely to say that they need time to prepare in some manner to confront their habit, expressing the other side of their ambivalence, namely the felt strength of their habit.

In contrast, for non-smokers to say they are planning to smoke in six months is a rejection of the influences that are inhibiting behavior change and to state an intention that is in the same direction as the reinforcing aspects of the habit. Therefore the statement that they plan to begin is not a reflection of an internal state of ambivalence, but of resolution. In other words, barring other barriers to action (e.g. unavailability of the substance), there is little reason for this person, once he has developed and recognized his intention to act, to wait six months to begin. 
This analysis suggests not that a period of ambivalence is absent in the acquisition of unhealthy behaviors, but that the items of the algorithm are not effective indicators of this stage. Items that assess not the intention to change behavior, but a questioning or weakening of the prohibitionary forces are more likely to clearly illuminate a Contemplation stage. It is unclear at this point whether this can be done using one item in an algorithm. A more promising route is using a URICA type scale instrument designed to expressly assess these attitudes. Unfortunately this is a more complicated and costly method of stage measurement.

Similarly, the existence and measurement of a Preparation stage based on subjects expressing an immediate plan to change their behavior is unlikely for the acquisition of unhealthy behaviors. Previous research (Stern et al., 1987) suggests that more likely is a stage between Contemplation and Action that is characterized by behavioral experimentation. Trying out the behavior can be conceptualized as an attempt to test the inhibitory influences on their behavior, such as seeing if the behavior in question results in injury, addiction, feelings of guilt or shame, or interpersonal rejection. A new scale instrument could include items to assess such experimental behavior.

\section{EXTERNAL VALIDITY}

This research provided strong evidence of the validity of the Stages of Change as measured by the algorithm. These results also help characterize the Stages of Change. Additional validity evidence using other Transtheoretical Model constructs will be investigated in Studies III, IV, and V. The establishment of predictive validity of these stages, as has been established in other behavioral domains such as smoking cessation will require further research. Statistically significant results are important in establishing validity, but also clear patterns across the Stages of Change add to our confidence in the validity of the stages as measured in this study and will be included in the discussion.

A set of three scales was developed in this study to provide a reliable measure of an important outcome variable. The scales of the Alcohol-Related Experiences instrument assess 
the risks inherent in immoderate drinking. Although problem lists have been commonly used in previous research, the present instrument provides a parsimonious set of three scales, that group related risk together. Furthermore the Distress scale assesses a dimension of risks that is under-investigated, but likely important, namely the emotional effects of alcohol use. This dimension is likely to be especially important to the investigation of immoderate drinking by women as they often score lower on more traditional problem lists.

\section{Stages of Cessation}

As this study aimed to measure the Stages of Change for immoderate alcohol use, which is conceptualized as use of alcohol that entails the increased risk of short-term negative sequelae, the strongest validity evidence is provided by the Alcohol-Related Experiences scales. The pattern of results are similar across the three scales. The slight but consistent rise in these scales between Precontemplation and Contemplation might be due to an increased recognition by Contemplators of the problems that alcohol is causing. As mentioned in the results section, this seems likely to be the reason for the peak on the Distress scale in Preparation, which is a scale likely to be more susceptible to the effects of intentional set. The results show that Precontemplators are over twice as high on the Excess and Distress scales as Maintainers, and over 4 times as high on the Problem scale. Overall these results demonstrate a strong relationship between the Stages of Cessation and the Alcohol-Related Experience scales.

The alcohol consumption variables also show a strong relationship to stage. Since these variables are more proximal to the stage definitions, the clear relationships observed are both more expected and more necessary to the validity of the stages.

There were only a few significant differences on the demographic variables. Women were more stage advanced than men, as were upperclassmen when compared to underclassmen. The gender differences are typical. Of health related behaviors previously studied, only in exercise does a higher proportion of men have a healthier lifestyle. The 
differences between upper and underclassmen reflects the general tendency for college students to "age" out of immoderate drinking patterns.

There was a general lack of significant findings for the psychosocial development scales across the Stage of Cessation. This does not seriously challenge the validity of these stages as examining these variables was exploratory in nature, but significant results would have added to the characterization of the stages. In addition to methodological explanations for these non-significant findings, it might be that high levels of relapse lead to stage members being more heterogeneous on variables such as measures of psychosocial maturity. Students who are progressing through the stages for first time might be less "mature" than those who are doing so after a number of failures even if in an earlier stage.

Further exploration of the relationship of stages to psychosocial development might find more significant resuits, but it is likely that a more fine grained analysis will be necessary. Understanding how these and other personality variables interact with the Stages of Change would usefully link model research to other psychological research efforts and would allow for the exploration of how personality modifies the process of behavior change as described in the Transtheoretical Model.

\section{Stages of Acquisition}

The external validity evidence clearly separated Precontemplation from the stages of Action and Maintenance. These differences are as expected in examining groups distinguished clearly on the amount that they drank. On average Precontemplators drank 7.3 drinks in a month, whereas those in Action drank 49.8 drinks, and those in Maintenance drank 80.1 drinks per month (These figures were calculated by multiplying Days by Drinks). Besides drinking much less, Precontemplators were more likely to be living with their parents, and had scored much lower than the other stages on the Alcohol-Related Experiences scales. 
Of greater interest perhaps is the differences between those in the Action and Maintenance stages of acquisition. Differences in demographic variables suggest that new acquisition in college is most likely to occur to those in their freshman year, who live in dormitories and who are female. This group is also about halfway between Precontemplators and Maintainers on the Alcohol-Related Experiences scales. Although these variables only incompletely characterize the possible differences between the Action and Maintenance groups, they do suggest that these groups have significant differences, and that interventions to reduce their drinking should take these differences into consideration. Certainly the lower level of drinking in Action suggests that one component of an intervention with the Action group could be prevention of additional increases in their drinking, along with attempts to encourage a reduction of their present drinking. Only more intensive research will determine on what important treatment related variables they differ, and how stage matched treatment would be designed.

In this study the criterion of six months for the acquisition of drinking was used. This figure has been used with great success in other behavioral domains, and was originally arrived at by careful research on the cessation of smoking. To the extent that the acquisition of unhealthy behavior is a novel area of inquiry, it is unclear the six month criterion is appropriate. This question would be most appropriately answered with longitudinal data that assesses the stability of immoderate drinking after varying lengths of time. In the absence of such extensive data, the clear differences between the Action and Maintenance groups on the validity variables are strong evidence that six months is an effective demarcation.

\section{SUMMARY}

The algorithm used in the present study assigned students into five Stages of Cessation and initial results attest to their validity. This staging method is easily administered, is easily analyzed, and results in a very small number of unstaged subjects. Furthermore, if only the Stages of Cessation are to be assessed, the item set can be reduced to four items. 
The algorithm was not effective in measuring Stages of Acquisition. It is hypothesized that a different approach is needed to assess states of ambivalence in the acquisition of unhealthy behaviors and that a scale measure might more effectively accomplish this. 


\section{REFERENCES}

Babor, T. F., Kranzler, H. R., \& Lauerman, R. J. (1987). Social Drinking as a Health and Psychosocial Risk Factor: Anstie’s Limit revisited. In M. Galanter (Ed.), Recent Developments in alcoholism (pp. 373-402). New York: Plenum.

Baer, J. S., Stacy, A., \& Larimer, M. (1991). Biases in the perception of drinking norms among college students. Journal of Studies on Alcohol, $\underline{52}, 580-586$.

Berkowitz, A. D. \& Perkins, H. W. (1986). Problem drinking among college students: A review of recent research. College Health, $\underline{35}, 21-28$.

Cattell, R, B. (1966). The Scree test for the number of factors. Multivariate Behavioral Research, 1 , 245-276.

Chickering, A. W. (1969). Education and Identity. San Francisco: Josset-Bass.

Comrey, A. L. (1988). Factor-analytic methods of scale development in personality and clinical psychology. Journal of Consulting and Clinical Psychology, 56, 754-761.

DiClemente, C. C. \& Hughes, S. O. (1990). Stages of change profiles in outpatient alcoholism treatment. Journal of Substance Abuse, 2 , 217-235.

DiClemente, C. C., Prochaska, J. O., Fairhurst, S. K., Velicer, W. F., Velasquez, M. M., \& Rossi, J. S. (1991). The process of smoking cessation: An analysis of precontemplation, contemplation and preparation stages of change. Journal of Consulting and Clinical Psychology, 59, 295-304.

DiClemente, C. C., Prochaska, J. O., \& Gilbertini, M. (1985). Self-efficacy and the stages of self change of smoking. Cognitive Therapy and Research, 9, 181-200.

Elder, J. P., De Moor, C., Young, R. L., Wildey, C. A., Molgaard, C. A., Golbeck, A. L., Sallis, J. F., \& Stern, R. A. (1990). Stages of adolescent tobacco-use acquisition. Addictive Behaviors, $15,449-454$.

Engs, R. (1977). Drinking patterns and drinking problems of college students. Journal of Studies on Alcohol, 38, 2144-2156. 
Fillmore, K. M. \& Midanik, L. (1984). Chronicity of drinking problems among men: A longitudinal study. Journal of Studies on Alcohol, 45, 228-236.

Harford, T. C. (1984). Situational factors in drinking: A developmental perspective on drinking contexts. In P. M. Miller \& T. D. Nirenberg (Eds.), Prevention of alcohol abuse (pp. 119-156). New York: Plenum Press.

Horn, J. L. (1965), A rationale and test for the number of factors in factor analysis. Psychometrika, 30, 179-185.

Jackson, D. N. (1970). A sequential system for personality scale development. In Spielberger (Ed.), Current topics in clinical and community psychology (Vol. 2, pp. 61-96). New York: Academic Press.

Jessor, R. (1987). Problem-behavior theory, psychosocial development, and adolescent problem drinking. British Journal of the Addictions, $\underline{82}, 331-342$.

Johnston, J. D., O'Malley, P. M., \& Bachman, J. G. (1992). Smoking, drinking, and illicit drug use among American secondary school students, college students, and young adults, 1975-1991 (Vol. 2). Washington, D.C.: National Institute on Drug Abuse.

Kraft, D. P. (1988). The prevention and treatment of alcohol problems on a college campus. Journal of Alcohol and Drug Education, 34, 37-51.

Laforge, R. G., Rossi, J. S., \& Migneault, J. P. (1994, April). Predicting intention to change high risk drinking practices. Poster presented at the 15 th annual conference of the Society of Behavioral Medicine, Boston, MA.

Lautenschlager, G. J. (1989). A comparison of alternatives to conducting Monte carlo analyses for determining parallel analysis criteria. Multivariate Behavioral Research, $\underline{24}, 365-395$.

Marcus, B. H., Rossi, J. S., Selby, V. C., Niaura, R. S., \& Abrams, D. B. (1992). The stages and processes of exercise adoption and maintenance. Health Psychology, 11, 386-395. 
McConnaughy, E. A., DiClemente, C. C., Prochaska, J. O., \& Velicer, W. F. (1989). Stages of change in psychotherapy: A follow-up report. Psychotherapy, 26, 494-503. McConnaughy, E. A., Prochaska, J. O., \& Velicer, W. F. (1982). Stages of change in psychotherapy: Measurement and sample profiles. Psychotherapy: Theory, Research and Practice, 20, 368-375.

Mayfield, D., McLeod, G., \& Hall, P. (1974). The CAGE questionnaire: Validation of a new alcoholism screening instrument. American Journal of Psychiatry, 131, 1121-1123.

Meilman, P. W., Stone, J. E., Gaylor, M. S., \& Turco, J. H. (1990). Alcohol consumption by college undergraduates: Current use and 10-year trends. Journal of Studies on Alcohol, 51, 389-395.

Migneault, J. P., Pallonen, U. E., \& Velicer, W. F. (1994). Stage and decisional balance for adolescent alcohol use. Paper submitted for presentation at the 15 th annual meeting of the Society of Behavioral Medicine, Boston, MA.

Migneault, J. P., Stevenson, J. F., \& Velicer, W. F. (1994, April). A validation study of the stages of change for risky drinking in college. Poster presented at the 15 th annual meeting of the Society of Behavioral Medicine in Boston, MA.

Migneault, J. P. (1992). A validation study of stages of change for risky drinking. Unpublished master's thesis, University of Rhode Island, Kingston, RI.

Pallonen, U. E., Murray, D. M., Schmid, L., Pirie, P., \& Luepker, R. (1990). Patterns of self-initiated smoking cessation among young adults. Health Psychology, 9 , 418-426.

Prochaska, J. O. \& DiClemente, C. C. (1983). Stages and processes of self-change of smoking: Toward an integrative model of change. Journal of Consulting and Clinical Psychology, 51, 390-395.

Prochaska, J. \& DiClemente, C. (1984). The transtheoretical approach: Crossing the traditional boundaries of therapy. Homewood, Illinois: Dow-Jones/Irwin. 
Prochaska, J. O., DiClemente, C. C., Velicer, W. F., Ginpil, S., \& Norcross, J. C. (1985). Predicting change in smoking status for self-changers. Addictive Behaviors, $\underline{10}, 395-$ 406.

Prochaska, J. O., DiClemente, C. C., Velicer, W. F., \& Rossi, J. S. (1993). Standardized, individualized, interactive and personalized self-help programs for stages of smoking cessation. Health Psychology, 12, 399-405.

Prochaska, J. O., Redding, C. A., Harlow, L. L., Rossi, J. S., \& Velicer, W. F. (in press). The transtheoretical model of change and HIV prevention. Health Education Quarterly.

Prochaska, J. O., Rossi, J. S., \& Wilcox, N. S. (1991). Change processes and psychotherapy outcomes in integrative case research. Journal of Psychotherapy Integration, 1(2), 103-119.

Prochaska, J. O., Velicer, W. F., Rossi, J. S., Goldstein, M. G., Marcus, B. H., Rakowski, W., Fiore, C., Harlow, L. L., Redding, C. A., Rosenbloom, D., \& Rossi, S. R. (1994). Stages of change and decisional balance for twelve problem behaviors. Health Psychology, 13, 37-46.

Rollnick, S., Heather, N., Gold, R., \& Hall, W. (1992). Development of a short 'readiness to change' questionnaire for use in brief, opportunistic interventions among excessive drinkers. British Journal of the Addictions, $\underline{87}, 743-754$.

Rosenbloom, D. (1991). A transtheoretical analysis of change among cocaine users. Unpublished doctoral dissertation, University of Rhode Island, Kingston, RI. Rossi, J. S. (1989). Exploring behavioral approaches to UV risk reduction.

Rossi, S. R., Rossi, J. S., Prochaska, J. O., \& Velicer, W. F. (1992). Application of the Transtheoretical Model of Behavior Change to Dietary Fat, (Abstract). International Journal of Psychology, 27, 628. 
Saunders, J. B. \& Aasland, O. G. (1987). WHO collaborative project on the identification and treatment of persons with harmful alcohol consumption: Report on Phase I: The development of a screening device (Vol. WHO Offset Publication 86.3). Geneva: World Health Organization.

Snow, M. G. (1991). A transtheoretical analysis of strategies in the recovery process from alcohol problems. Unpublished doctoral dissertation, University of Rhode Island, Kingston, RI.

Stern, R. A., Prochaska, J. O., Velicer, W. F., \& Elder, J. P. (1987). Stages of adolescent cigarette smoking acquisition: Measurement and sample profiles. Addictive Behaviors, $\underline{12}, 319-329$.

Velicer, W. F. (1976). Determining the number of components from the matrix of partial correlations. Psychometrika, $\underline{41}, 321-327$.

Velicer, W. F., DiClemente, C. C., Rossi, J. S., \& Prochaska, J.O. (1990). Relapse situations and self-efficacy: An integrative model. Addictive Behaviors, 15, 271-283.

Wechsler, H., Davenport, A., Dowdall, G., Moeykens, B., \& Castillo, S. (1994). Health and behavioral consequences of binge drinking in college. Journal of the American Medical Association, 272, 1672-1677.

Wechsler, H. \& McFadden, M. (1979). Drinking among college students in New England. Journal of Studies on Alcohol, 40, 969-996.

White, H. R. \& Labouvie, E. W. (1989). Towards the assessment of adolescent problem drinking. Journal of Studies on Alcohol, 50, 30-37.

Winston, R. B. \& Miller, T. K. (1987). Student developmental task and lifestyle inventory manual. Athens, GA: Student Development Associates.

Zwick, W. R. \& Velicer, W. F. (1986). Comparison of five rules for determining the number of components to retain. Psychological Bulletin, $99_{\text {sy }}$ 432-442. 


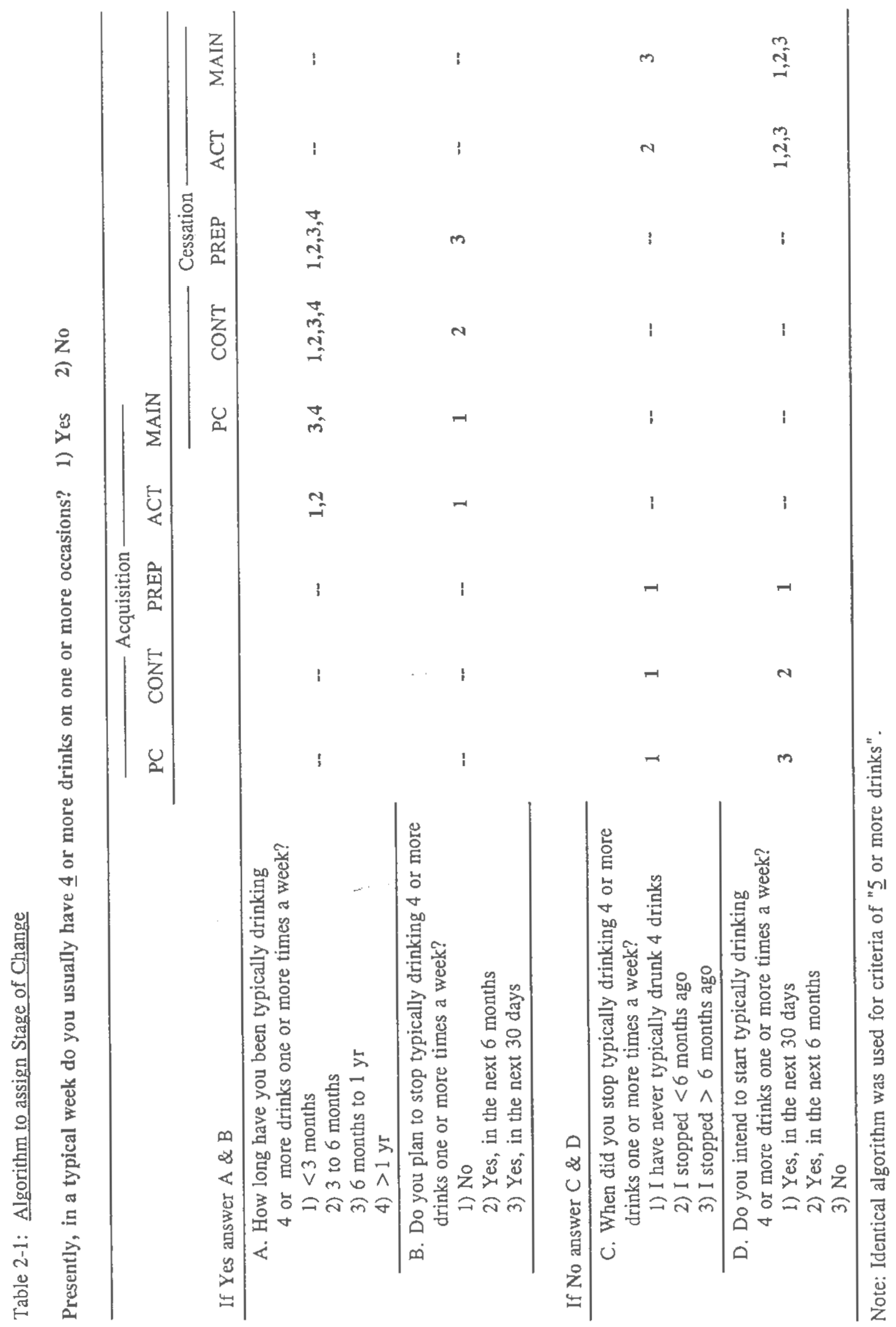


Table 2-2: Stage distributions by criteria

\begin{tabular}{|c|c|c|c|c|c|c|}
\hline \multirow[b]{3}{*}{ STAGE } & \multicolumn{6}{|c|}{4 or more drinks } \\
\hline & \multicolumn{2}{|c|}{ Total } & \multicolumn{2}{|c|}{ Women } & \multicolumn{2}{|c|}{ Men } \\
\hline & $\mathrm{N}$ & $\%$ & N & $\%$ & $\mathrm{~N}$ & $\%$ \\
\hline Precontemplation-Acquisition & 134 & 21.8 & 107 & 26.4 & 27 & 12.9 \\
\hline Action-Acquisition & 42 & 6.8 & 27 & 6.7 & 15 & 7.2 \\
\hline $\begin{array}{l}\text { Maintenance-Acquisition/ } \\
\text { Precontemplation }\end{array}$ & 234 & 38.1 & 130 & 32.1 & 104 & 49.8 \\
\hline Contemplation & 59 & 9.6 & 36 & 8.9 & 23 & 11.0 \\
\hline Preparation & 19 & 3.1 & 13 & 3.2 & 6 & 2.9 \\
\hline Action & 45 & 7.3 & 34 & 8.4 & 11 & 5.3 \\
\hline Maintenance & 81 & 13.2 & 58 & 14.3 & 23 & 11.0 \\
\hline
\end{tabular}

* Male/Female Differences Significant: $\chi^{2}(6)=26.54, p<.0001$

\begin{tabular}{|c|c|c|c|c|c|c|}
\hline \multirow[b]{3}{*}{ STAGE } & \multicolumn{6}{|c|}{5 or more drinks } \\
\hline & \multicolumn{2}{|c|}{ Total } & \multicolumn{2}{|c|}{ Women } & \multicolumn{2}{|c|}{ Men } \\
\hline & $\mathrm{N}$ & $\%$ & $\mathrm{~N}$ & $\%$ & $\mathrm{~N}$ & $\%$ \\
\hline Precontemplation-Acquisition & 195 & 32.1 & 152 & 37.9 & 43 & 20.9 \\
\hline Action-Acquisition & 25 & 4.1 & 17 & 4.2 & 8 & 3.9 \\
\hline $\begin{array}{l}\text { Maintenance-Acquisition/ } \\
\text { Precontemplation }\end{array}$ & 188 & 31.0 & 95 & 23.7 & 93 & 45.1 \\
\hline Contemplation & 52 & 8.6 & 29 & 7.2 & 23 & 11.2 \\
\hline Preparation & 16 & 2.6 & 8 & 2.0 & 8 & 3.9 \\
\hline Action & 47 & 7.7 & 38 & 9.5 & 9 & 4.4 \\
\hline Maintenance & 84 & 13.8 & 62 & 15.5 & 22 & 10.7 \\
\hline
\end{tabular}

* Male/Female Differences Significant: $\chi^{2}(6)=43.69, p<.0001$ 
Table 2-3: Cross-classification of stage using different consumption criteria by gender

\begin{tabular}{|c|c|c|c|c|c|c|c|}
\hline \multicolumn{8}{|c|}{ WOMEN } \\
\hline \multirow{2}{*}{$\begin{array}{l}\text { Staging criterion: } \\
4 \text { or more drinks }\end{array}$} & \multicolumn{7}{|c|}{ Staging criterion: 5 or more drinks } \\
\hline & 1 & 2 & 3 & 4 & 5 & 6 & 7 \\
\hline 1. Precontemplation-Acquisition & 105 & & & & & 1 & \\
\hline 2. Action-Acquisition & 8 & 14 & 1 & 1 & & 2 & \\
\hline $\begin{array}{l}3 \text { Maintenance-Acquisition/ } \\
\text { Precontemplation }\end{array}$ & 13 & 3 & 92 & 8 & & 4 & 8 \\
\hline 4. Contemplation & 3 & & & 20 & 2 & 6 & 5 \\
\hline 5. Preparation & 1 & & 1 & & 6 & 2 & 2 \\
\hline 6. Action & 6 & & & & & 23 & 5 \\
\hline 7. Maintenance & 16 & & & & & & 42 \\
\hline
\end{tabular}

\section{MEN}

\begin{tabular}{|c|c|c|c|c|c|c|c|}
\hline \multirow{2}{*}{$\begin{array}{l}\text { Staging criterion: } \\
4 \text { or more drinks }\end{array}$} & \multicolumn{7}{|c|}{ Staging criterion: 5 or more drinks } \\
\hline & 1 & 2 & 3 & 4 & 5 & 6 & 7 \\
\hline 1. Precontemplation-Acquisition & 26 & & 1 & & & & \\
\hline 2. Action-Acquisition & 2 & 8 & & 2 & 1 & & \\
\hline $\begin{array}{l}\text { 3. Maintenance-Acquisition/ } \\
\text { Precontemplation }\end{array}$ & 4 & & 90 & 5 & & 1 & \\
\hline 4. Contemplation & 5 & & 1 & 15 & 2 & & \\
\hline 5. Preparation & 1 & & & & 5 & & \\
\hline 6. Action & 2 & & & 1 & & 7 & 1 \\
\hline 7. Maintenance & 2 & & & & & 1 & 20 \\
\hline
\end{tabular}


Table 2-4: Final gender-specific stage distributions

\begin{tabular}{|c|c|c|c|c|c|c|}
\hline \multirow[b]{3}{*}{ STAGE } & \multicolumn{6}{|c|}{ Stage by gender specific criteria } \\
\hline & \multicolumn{2}{|c|}{ Total } & \multicolumn{2}{|c|}{ Women } & \multicolumn{2}{|c|}{ Men } \\
\hline & $\mathrm{N}$ & $\%$ & $\mathrm{~N}$ & $\%$ & $N$ & $\%$ \\
\hline Precontemplation-Acquisition & 150 & 24.5 & 107 & 26.4 & 43 & 20.9 \\
\hline Action-Acquisition & 35 & 5.7 & 27 & 6.7 & 8 & 3.9 \\
\hline $\begin{array}{l}\text { Maintenance-Acquisition/ } \\
\text { Precontemplation }\end{array}$ & 223 & 36.5 & 130 & 32.1 & 93 & 45.1 \\
\hline Contemplation & 59 & 9.7 & 36 & 8.9 & 23 & 11.2 \\
\hline Preparation & 21 & 3.4 & 13 & 3.2 & 8 & 3.9 \\
\hline Action & 43 & 7.0 & 34 & 8.4 & 9 & 4.4 \\
\hline Maintenance & 80 & 13.1 & 58 & 14.3 & 22 & 10.7 \\
\hline
\end{tabular}

* Male/Female differences significant: $\chi^{2}(6)=15.37, p<.05$ 
Table 2-5: Component loadings for Alcohol Related Experiences scales

Item stem: How many times in the last 12 months have you had the following experiences related to your alcohol consumption?

\section{Scale 1: Excess}

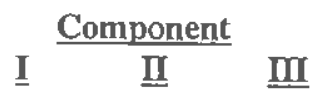

1. Been hungover $\quad .79$

2. Drank to excess $\quad .71$

3. Ridden with someone who was driving after drinking $\quad .68$

4. Gotten sick or vomited $\quad .68$

5. Missed class or had other academic problems $\quad .67$

6. Could not remember some amount of time $\quad .65$

7. Driven after drinking $\quad .64$

8. Passed out $\quad .64$

9. Gotten into an argument or fight $\quad .52$

10. Had unprotected sex $\quad .49$

\section{Scale 2: Distress}

1. Felt low, blue or bummed out the next day $\quad .79$

2. Felt guilty about your drinking $\quad .74$

3. Been emotionally upset $\quad .69$

4. Had a personality change $\quad .66$

5. Made an effort to cut down $\quad .62$

6. Hurt your health $\quad .50$

7. Been unable to stop drinking once you began .41

Scale 3: Problem

1. Needed a first drink in the morning $\quad .71$

2. Been angry at someone's suggestion that you cut down

3. Had job problems $\quad 66$

4. Been criticized by a date $\quad .64$

5. Gotten into trouble with school authorities, parents, or the law

6. Been injured or injured someone else $\quad .54$ 
Table 2-6: Means, standard deviations, coefficient alphas and scale intercorrelations for the Alcohol-Related Experiences Scales

\begin{tabular}{lcccccc}
\hline & & & & \multicolumn{3}{c}{ Correlations } \\
\cline { 5 - 7 } SCALE & Mean & SD & Alpha & Excess & Distress & Problem \\
\hline Excess & 1.87 & 1.17 & .88 & - & & \\
Distress & 1.12 & 1.06 & .84 & .44 & - & \\
Problem & .35 & 0.63 & .80 & .37 & .37 & -- \\
\hline
\end{tabular}

Note: All correlations significant at $\mathrm{p}<.01$ 
Table 2-7: Distributions of categorical variables across the Stages of Acquisition

\begin{tabular}{|c|c|c|c|c|c|c|}
\hline \multirow[b]{2}{*}{ STAGE } & \multicolumn{2}{|c|}{ Total } & \multicolumn{2}{|c|}{ Women } & \multicolumn{2}{|c|}{ Men } \\
\hline & $\mathbf{N}$ & $\%$ & $\mathrm{~N}$ & $\%$ & $\mathrm{~N}$ & $\%$ \\
\hline Precontemplation & 150 & 36.8 & 107 & 40.5 & 43 & 29.9 \\
\hline Action & 35 & 8.6 & 27 & 10.2 & 8 & 5.6 \\
\hline Maintenance & 223 & 54.6 & 130 & 49.2 & 93 & 64.6 \\
\hline
\end{tabular}

Note: Male/Female differences significant: $\chi^{2}(2)=9.27, \mathrm{p}<.05$

Living Situation

\begin{tabular}{|c|c|c|c|c|c|c|c|c|}
\hline \multirow[b]{2}{*}{ STAGE } & \multicolumn{2}{|c|}{ With Parents } & \multicolumn{2}{|c|}{ Dormitory } & \multicolumn{2}{|c|}{ Greek } & \multicolumn{2}{|c|}{ Off Campus } \\
\hline & $\mathrm{N}$ & $\%$ & $\mathrm{~N}$ & $\%$ & $\mathrm{~N}$ & $\%$ & $\mathrm{~N}$ & $\%$ \\
\hline Precontemplation & 36 & 56.5 & 75 & 43.1 & 8 & 18.2 & 28 & 23.0 \\
\hline Action & 4 & 6.3 & 25 & 14.4 & 2 & 4.6 & 4 & 3.3 \\
\hline Maintenance & 24 & 37.5 & 74 & 42.5 & 34 & 77.3 & 90 & 73.8 \\
\hline
\end{tabular}

Note: Differences across living situations are significant: $\chi^{2}(6)=51.34, p<.001$

\begin{tabular}{|c|c|c|c|c|c|c|c|c|}
\hline \multirow[b]{3}{*}{ STAGE } & \multicolumn{8}{|c|}{ Class } \\
\hline & \multicolumn{2}{|c|}{ Freshman } & \multicolumn{2}{|c|}{ Sophomore } & \multicolumn{2}{|c|}{ Junior } & \multicolumn{2}{|c|}{ Sentor } \\
\hline & $\mathrm{N}$ & $\%$ & $\mathrm{~N}$ & $\%$ & $\mathrm{~N}$ & $\%$ & $\mathrm{~N}$ & $\%$ \\
\hline Precontemplation & 32 & 39.5 & 59 & 41.0 & 30 & 40.0 & 23 & 25.8 \\
\hline Action & 18 & 22.2 & 9 & 6.3 & 3 & 4.0 & 4 & 4.5 \\
\hline Maintenance & 31 & 38.3 & 76 & 52.8 & 42 & 56.0 & 62 & 69.7 \\
\hline
\end{tabular}

Note: Differences across classes are significant: $\chi^{2}(6)=33.4, p<.001$ 
Table 2-8: Means and standard deviations for continuous variables across the Stages of Acquisition

\begin{tabular}{|c|c|c|c|c|c|c|c|c|}
\hline \multirow[b]{3}{*}{ STAGE } & & & \multicolumn{6}{|c|}{ Consumption Variables } \\
\hline & \multicolumn{2}{|c|}{ AGE } & \multicolumn{2}{|c|}{ DAYS } & \multicolumn{2}{|c|}{ DRINKS } & \multicolumn{2}{|c|}{ INTOX } \\
\hline & Mean & SD & Mean & SD & Mean & SD & Mean & $\mathrm{SD}$ \\
\hline Precontemplation & 20.33 & 1.65 & 3.14 & 3.83 & 2.31 & 1.93 & 3.99 & 1.98 \\
\hline Action & 19.52 & 1.10 & 9.03 & 4.42 & 5.51 & 1.96 & 5.11 & 1.72 \\
\hline Maintenance & 20.48 & 1.43 & 11.78 & 5.70 & 6.80 & 3.14 & 6.72 & 2.41 \\
\hline
\end{tabular}

\begin{tabular}{|c|c|c|c|c|c|c|}
\hline \multirow[b]{3}{*}{ STAGE } & \multicolumn{6}{|c|}{ Alcohol Related Experiences } \\
\hline & \multicolumn{2}{|c|}{ EXCESS } & \multicolumn{2}{|c|}{ DISTRESS } & \multicolumn{2}{|c|}{ PROBLEM } \\
\hline & Mean & SD & Mean & $\mathrm{SD}$ & Mean & $\mathrm{SD}$ \\
\hline Precontemplation & .54 & .62 & .36 & .51 & .07 & .20 \\
\hline Action & 1.86 & .91 & .98 & .94 & .23 & .40 \\
\hline Maintenance & 2.49 & 1.02 & 1.41 & 1.12 & .50 & .74 \\
\hline
\end{tabular}

\begin{tabular}{|c|c|c|c|c|c|c|}
\hline \multirow[b]{3}{*}{ STAGE } & \multicolumn{6}{|c|}{ Psychosocial Development } \\
\hline & \multicolumn{2}{|c|}{$\begin{array}{c}\text { PEER } \\
\text { RELATIONS }\end{array}$} & \multicolumn{2}{|c|}{$\begin{array}{l}\text { EMOTIONAL } \\
\text { AUTONOMY }\end{array}$} & \multicolumn{2}{|c|}{$\begin{array}{l}\text { LIFESTYLE } \\
\text { PLANNING }\end{array}$} \\
\hline & Meán & SD & Mean & $\mathrm{SD}$ & Mean & SD \\
\hline Precontemplation & 3.35 & .55 & 3.14 & .64 & 3.41 & .65 \\
\hline Action & 3.31 & .57 & 2.95 & .72 & 3.09 & .65 \\
\hline Maintenance & 3.36 & .51 & 3.21 & .57 & 3.14 & .63 \\
\hline
\end{tabular}


Table 2-9: Distributions of categorical variables across Stage of Cessation

\begin{tabular}{|c|c|c|c|c|c|c|}
\hline \multirow[b]{2}{*}{ STAGE } & \multicolumn{2}{|c|}{ Total } & \multicolumn{2}{|c|}{ Women } & \multicolumn{2}{|c|}{ Men } \\
\hline & $\mathrm{N}$ & $\%$ & $N$ & $\%$ & N & $\%$ \\
\hline Precontemplation & 223 & 52.4 & 130 & 48.0 & 93 & 60.0 \\
\hline Contemplation & 59 & 13.8 & 36 & 13.3 & 23 & 14.8 \\
\hline Preparation & 21 & 4.9 & 13 & 4.8 & 8 & 5.2 \\
\hline Action & 43 & 10.1 & 34 & 12.6 & 9 & 5.8 \\
\hline Maintenance & 80 & 18.8 & 58 & 21.4 & 22 & 14.2 \\
\hline
\end{tabular}

Note: Male/Female differences significant: $\chi^{2}(4)=10.09, p<.05$

\begin{tabular}{|c|c|c|c|c|c|c|c|c|}
\hline \multirow[b]{3}{*}{ STAGE } & \multicolumn{8}{|c|}{ Living Situation } \\
\hline & \multicolumn{2}{|c|}{ Dormitory } & \multicolumn{2}{|c|}{ Greek } & \multicolumn{2}{|c|}{ Off Campus } & \multicolumn{2}{|c|}{ With Parents } \\
\hline & $\mathrm{N}$ & $\%$ & $\mathrm{~N}$ & $\%$ & $\mathrm{~N}$ & $\%$ & $\mathbf{N}$ & $\%$ \\
\hline Precontemplation & 74 & 50.0 & 34 & 60.7 & 90 & 52.9 & 24 & 53.3 \\
\hline Contemplation & 18 & 12.2 & 11 & 19.6 & 26 & 15.3 & 3 & 6.8 \\
\hline Preparation & 11 & 7.4 & 2 & 3.6 & 6 & 3.5 & 1 & 2.2 \\
\hline Action & 19 & 12.8 & 2 & 3.6 & 14 & 8.2 & 7 & 15.6 \\
\hline Maintenance & 26 & 17.6 & 7 & 12.5 & 34 & 20.0 & 10 & 22.2 \\
\hline
\end{tabular}

Note: Differences across living situations are non-significant

\begin{tabular}{|c|c|c|c|c|c|c|c|c|}
\hline \multirow[b]{3}{*}{ STAGE } & \multicolumn{8}{|c|}{ Class } \\
\hline & \multicolumn{2}{|c|}{ Freshman } & \multicolumn{2}{|c|}{ Sophomore } & \multicolumn{2}{|c|}{ Junior } & \multicolumn{2}{|c|}{ Senior } \\
\hline & $N$ & $\%$ & $N$ & $\%$ & $\mathrm{~N}$ & $\%$ & $\mathrm{~N}$ & $\%$ \\
\hline Precontemplation & 31 & 54.4 & 76 & 58.5 & 42 & 52.5 & 62 & 46.3 \\
\hline Contemplation & 7 & 12.3 & 14 & 10.8 & 10 & 12.5 & 25 & 18.7 \\
\hline Preparation & 5 & 8.8 & 8 & 6.2 & 2 & 2.5 & 5 & 3.7 \\
\hline Action & 9 & 15.8 & 12 & 9.2 & 10 & 17.5 & 11 & 8.2 \\
\hline Maintenance & 5 & 8.8 & 20 & 15.4 & 16 & 20.0 & 31 & 23.1 \\
\hline
\end{tabular}

Note: Differences across classes are non-significant 
Table 2-10: Means and standard deviations for continuous variables across the Stages of Cessation

\begin{tabular}{|c|c|c|c|c|c|c|c|c|}
\hline \multirow[b]{3}{*}{ STAGE } & \multirow{2}{*}{\multicolumn{2}{|c|}{ AGE }} & \multicolumn{6}{|c|}{ Consumption Variables } \\
\hline & & & \multicolumn{2}{|c|}{ DAYS } & \multicolumn{2}{|c|}{ DRINKS } & \multicolumn{2}{|c|}{ INTOX } \\
\hline & Mean & $\overline{\mathrm{SD}}$ & Mean & $\mathrm{SD}$ & Mean & $\overline{S D}$ & Mean & $\mathrm{SD}$ \\
\hline Precontemplation & 20.48 & 1.43 & 11.78 & 5.70 & 6.80 & 3.13 & 6.23 & $2 . \overline{41}$ \\
\hline Contemplation & 20.68 & 1.15 & 11.10 & 5.97 & 6.48 & 3.33 & 5.97 & 2.02 \\
\hline Preparation & 20.19 & 1.46 & 10.52 & 6.37 & 6.05 & 2.54 & 6.52 & 2.25 \\
\hline Action & 20.30 & 1.52 & 5.07 & 3.02 & 4.45 & 1.99 & 5.05 & 2.05 \\
\hline Maintenance & 21.64 & 1.98 & 4.22 & 3.45 & 2.91 & 1.70 & 4.39 & 1.81 \\
\hline
\end{tabular}

\begin{tabular}{|c|c|c|c|c|c|c|}
\hline \multirow[b]{3}{*}{ STAGE } & \multicolumn{6}{|c|}{ Alcohol Related Experiences } \\
\hline & \multicolumn{2}{|c|}{ EXCESS } & \multicolumn{2}{|c|}{ DISTRESS } & \multicolumn{2}{|c|}{ PROBLEM } \\
\hline & Mean & $\mathrm{SD}$ & Mean & $S D$ & Mean & $\mathrm{SD}$ \\
\hline Precontemplation & 2.49 & 1.02 & 1.41 & 1.12 & .50 & .74 \\
\hline Contemplation & 2.59 & 1.01 & 1.61 & 1.17 & .60 & .83 \\
\hline Preparation & 2.34 & .87 & 1.96 & 1.26 & .48 & .62 \\
\hline Action & 1.57 & .98 & 1.12 & .92 & .22 & .31 \\
\hline Maintenance & 1.11 & .86 & .59 & .77 & .12 & .42 \\
\hline
\end{tabular}

\begin{tabular}{|c|c|c|c|c|c|c|}
\hline \multirow[b]{3}{*}{ STAGE } & \multicolumn{6}{|c|}{ Psychosocial Maturity } \\
\hline & \multicolumn{2}{|c|}{$\begin{array}{c}\text { PEER } \\
\text { RELATIONS }\end{array}$} & \multicolumn{2}{|c|}{$\begin{array}{l}\text { EMOTIONAL } \\
\text { AUTONOMY }\end{array}$} & \multicolumn{2}{|c|}{$\begin{array}{l}\text { LIFESTYLE } \\
\text { PLANNING }\end{array}$} \\
\hline & Mean & $\mathrm{SD}$ & Mean & SD & Mean & SD \\
\hline Precontemplation & 3.36 & .51 & 3.21 & .57 & 3.14 & .63 \\
\hline Contemplation & 3.33 & .56 & 3.19 & .59 & 3.00 & .60 \\
\hline Preparation & 3.36 & .48 & 2.98 & .63 & 3.15 & .80 \\
\hline Action & 3.27 & .53 & 3.07 & .53 & 3.29 & .56 \\
\hline Maintenance & 3.46 & .50 & 3.29 & .62 & 3.36 & .76 \\
\hline
\end{tabular}


Figure 2-1: Distribution of Days by gender

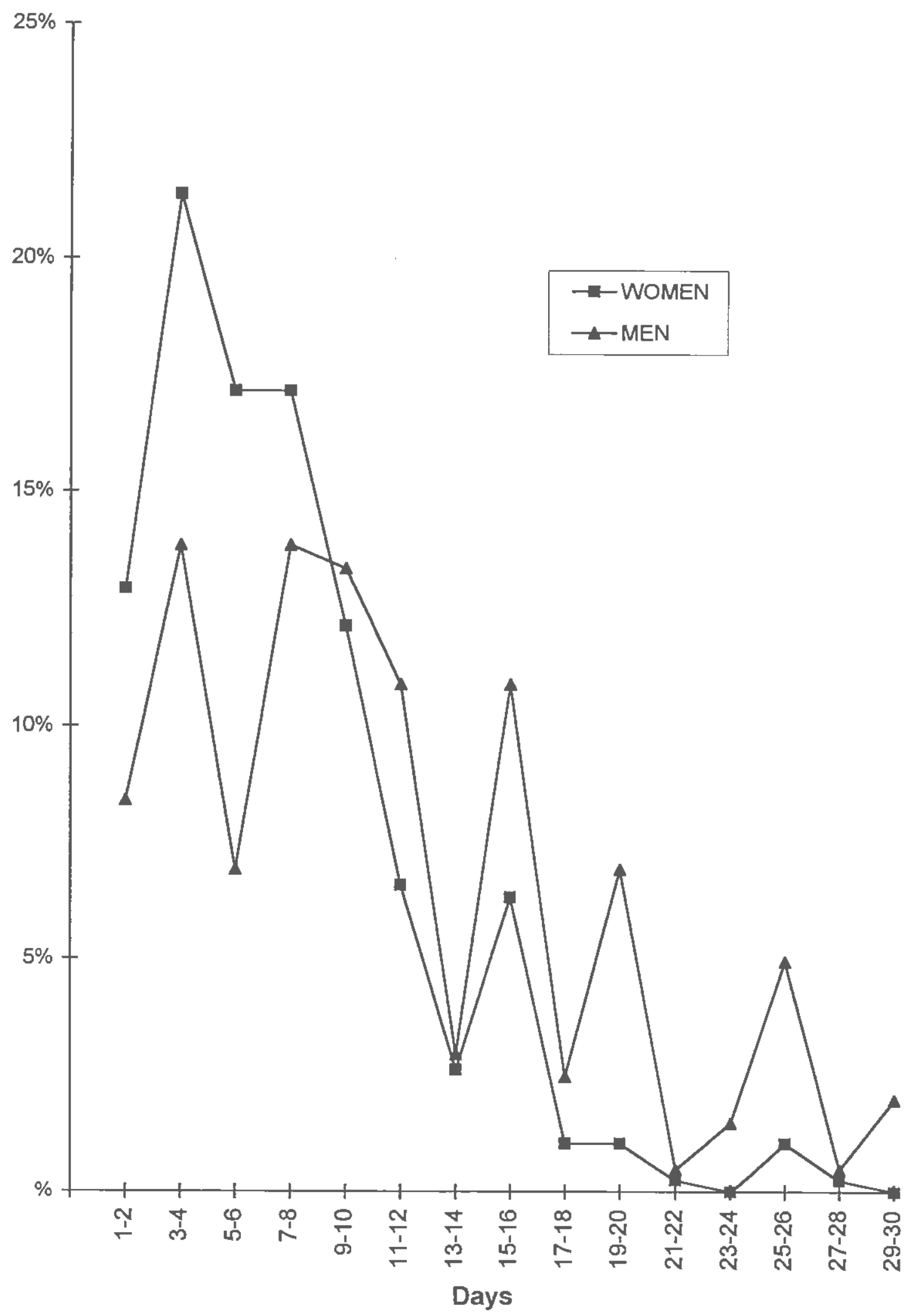


Figure 2-2: Distribution of Drinks by gender

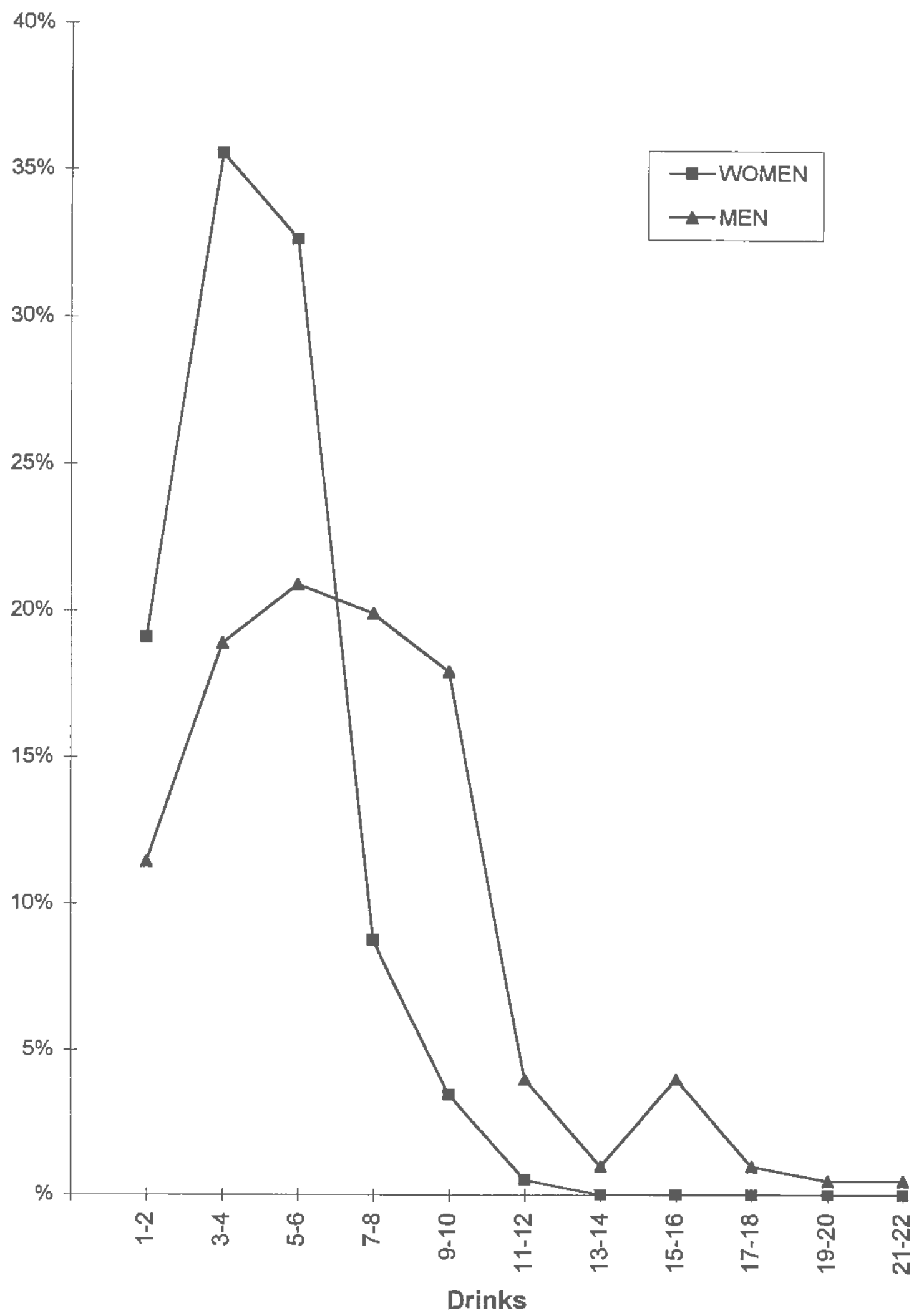


Figure 2-3: Age by Stage of Acquisition

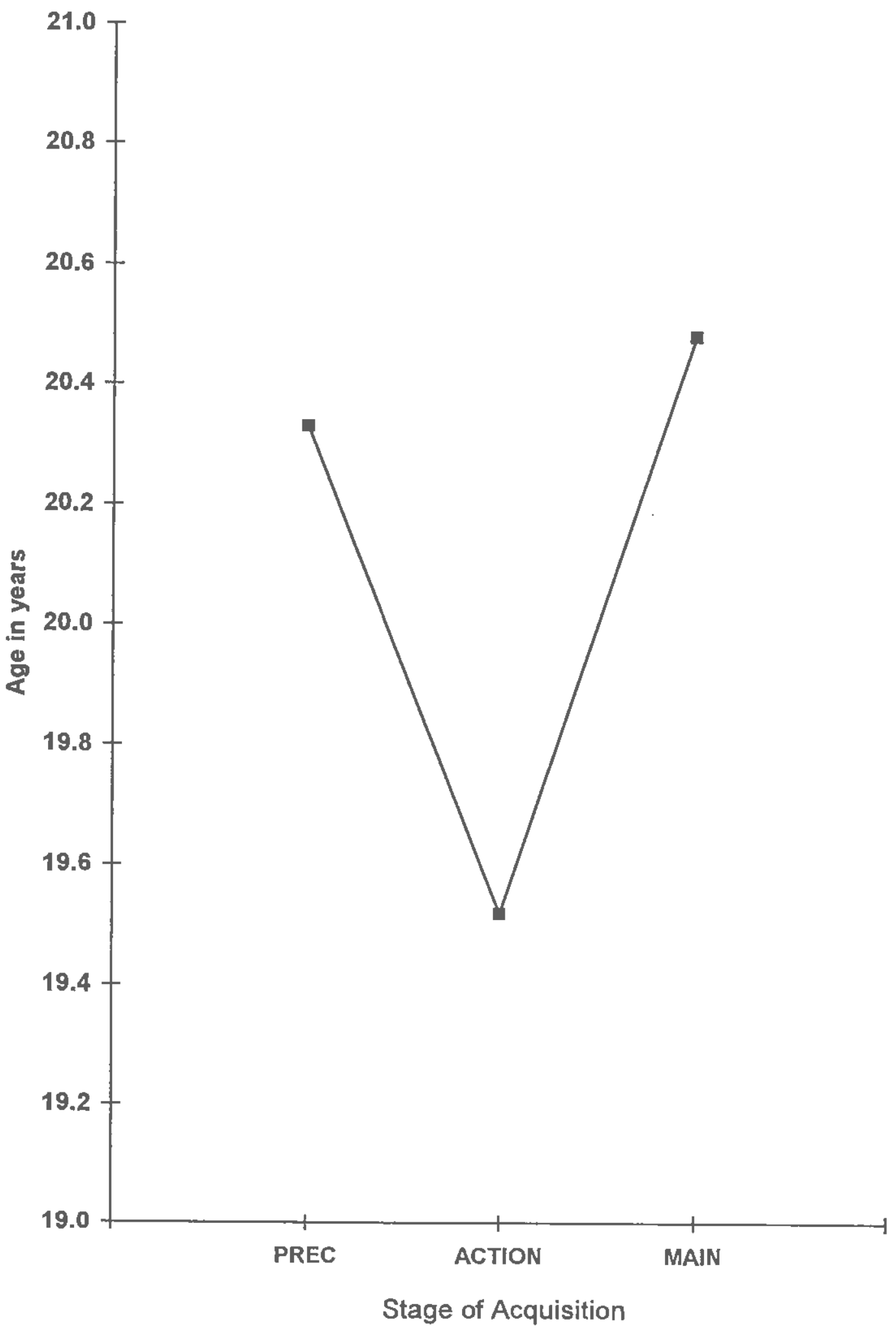


Figure 2-4: Consumption variables by Stage of Acquisition

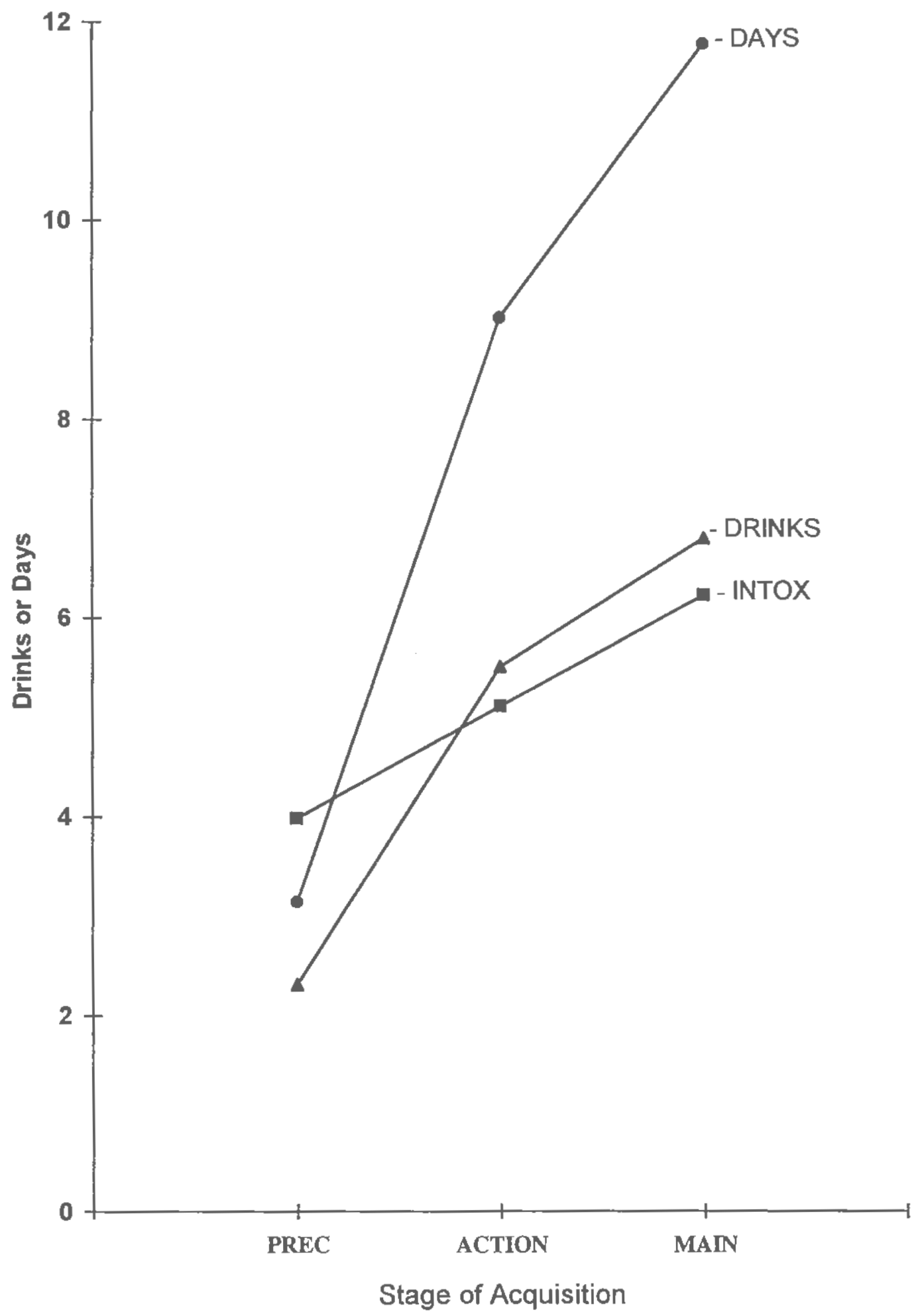


Figure 2-5: Alcohol-Related Experiences scales by Stage of Acquisition

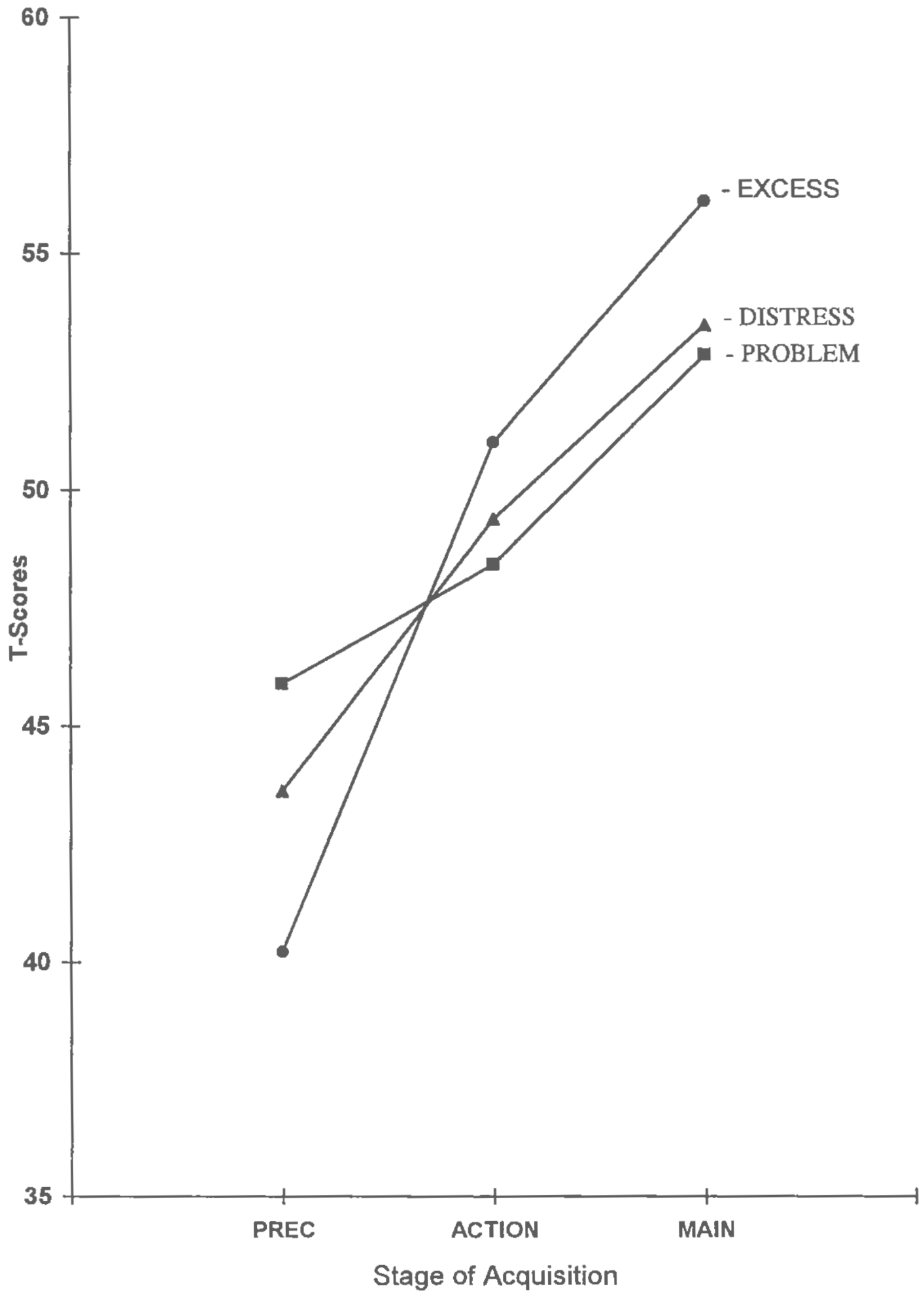


Figure 2-6: Psychosocial development scales by Stage of Acquisition

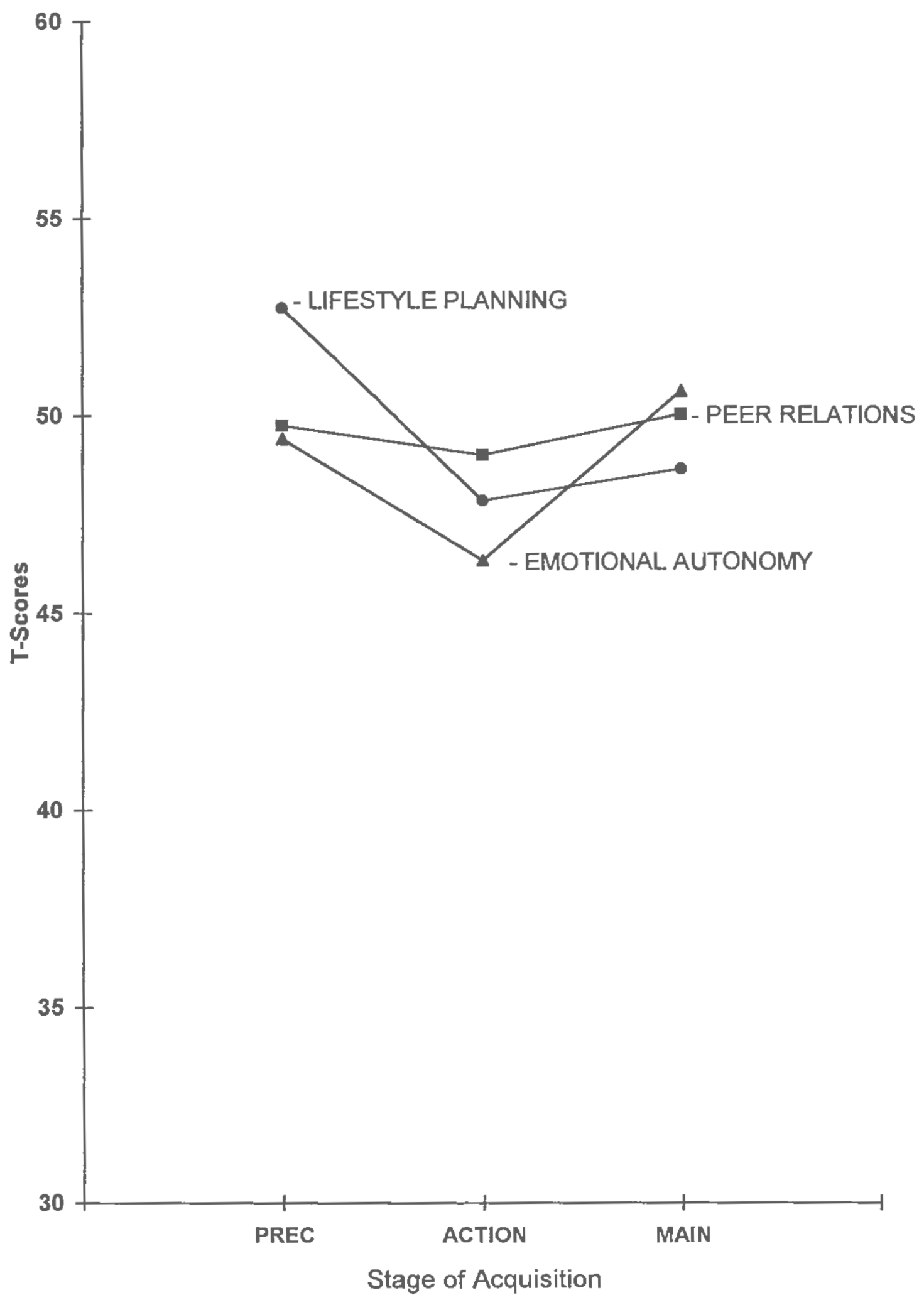


Figure 2-7: Age by Stage of Cessation

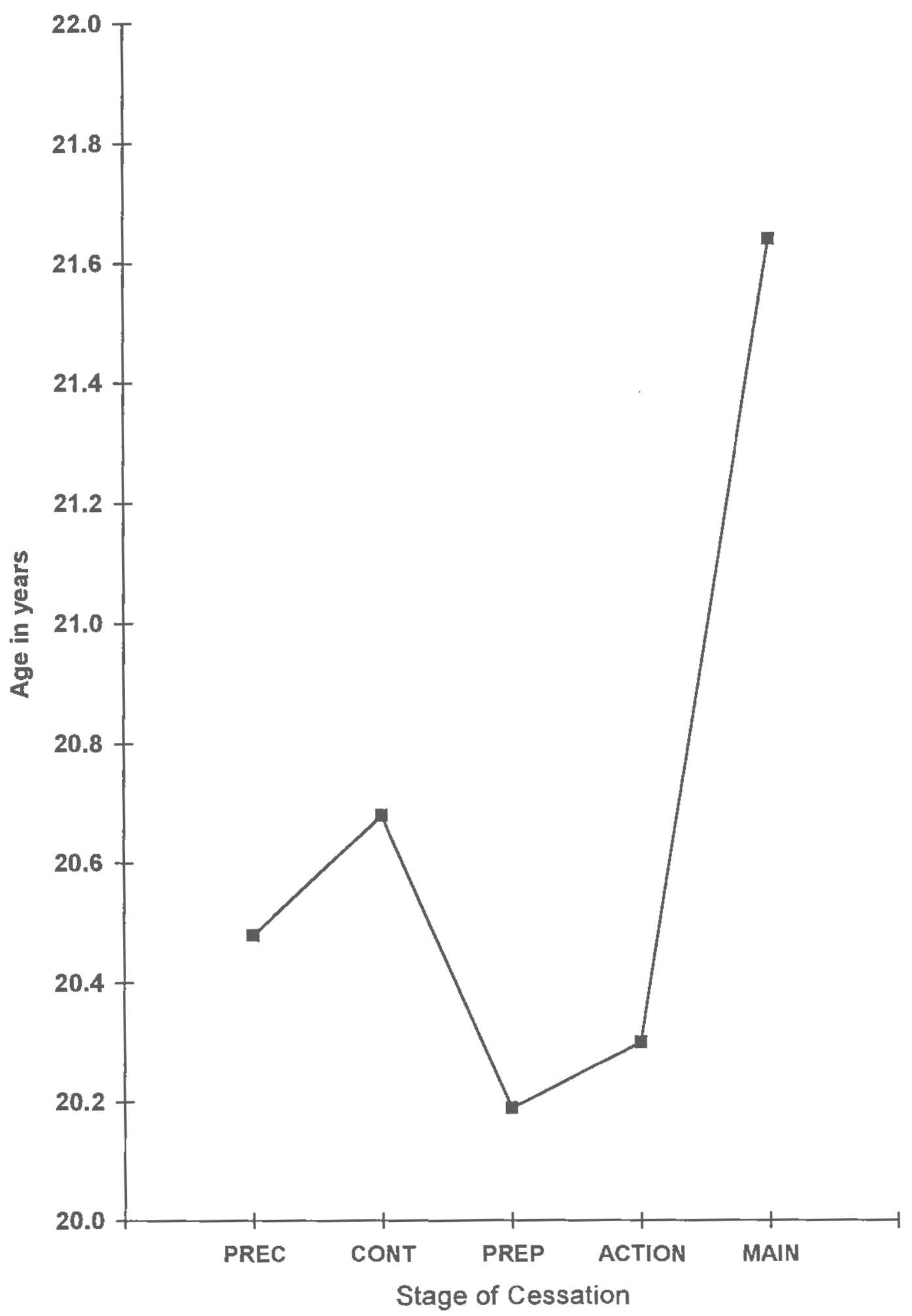


Figure 2-8: Consumption variables by Stage of Cessation

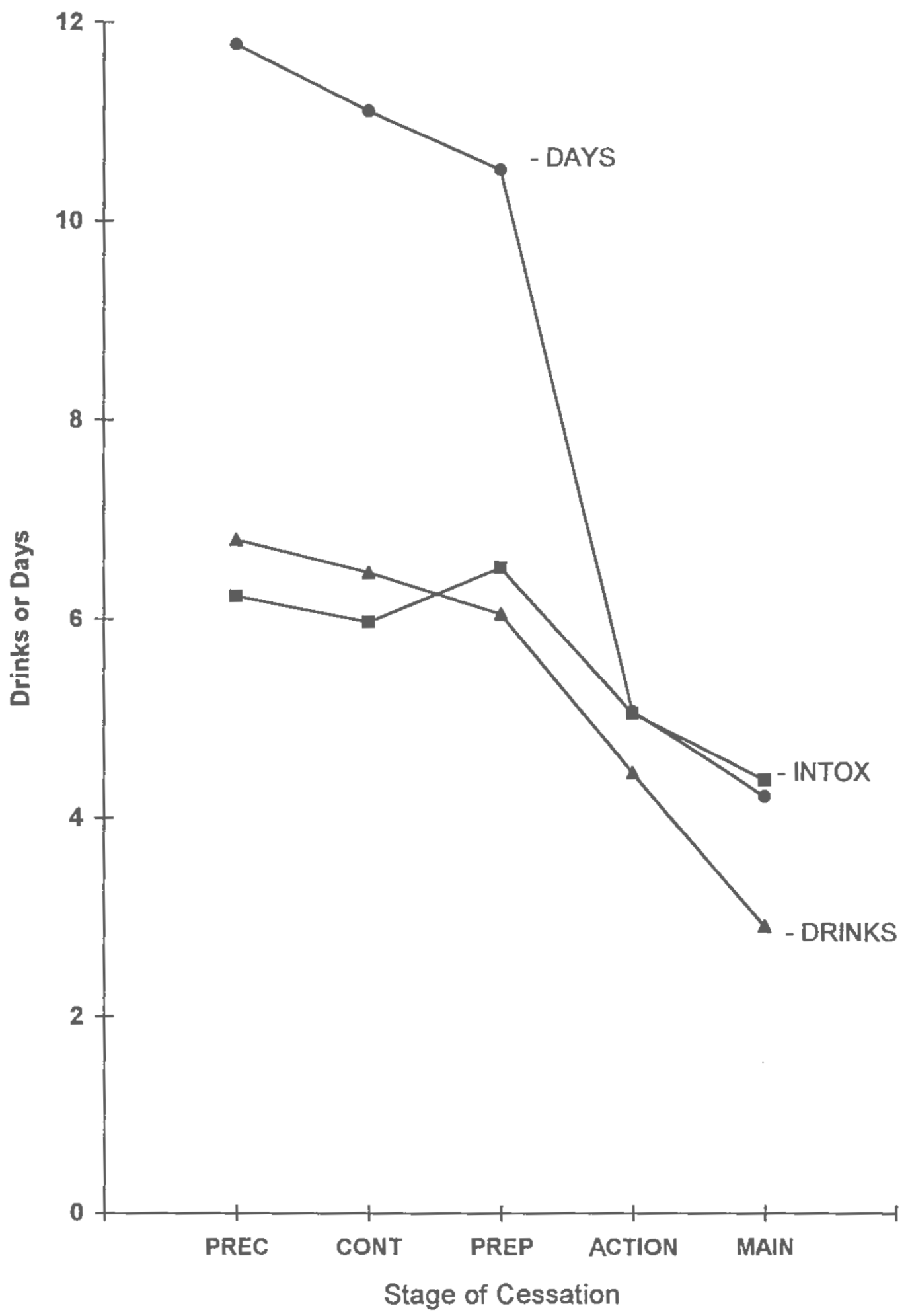


Figure 2-9: Alcohol-Related Experiences scales by Stage of Cessation

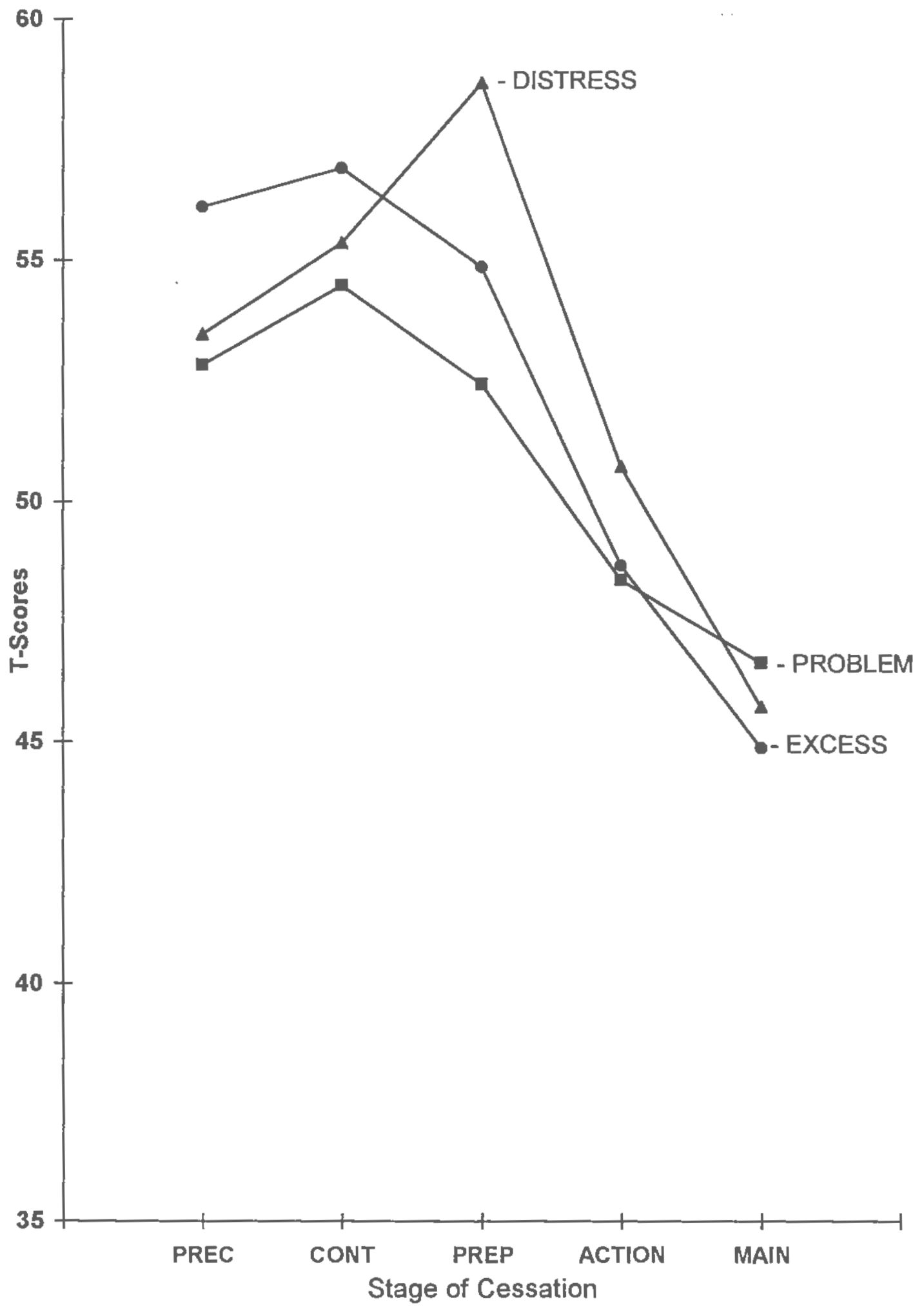


Figure 2-10: Psychosocial development scales by Stage of Cessation

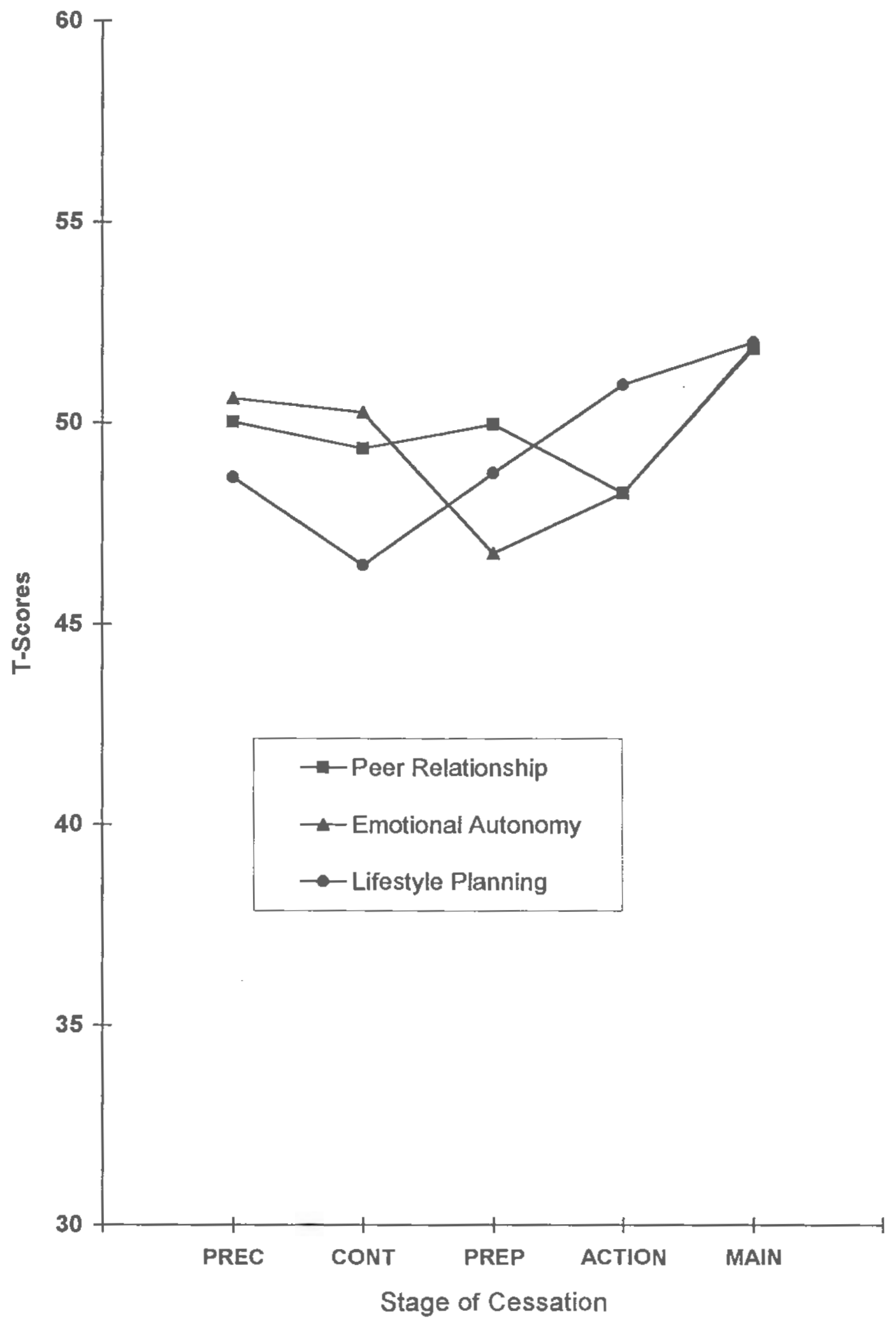




\section{PART III, STUDY 2: URICA-A SCALE INSTRUMENT FOR MEASURING STAGE OF CHANGE FOR IMMODERATE ALCOHOL USE}

\section{INTRODUCTION}

Alcohol consumption by college students is a very complex behavior involving almost all students, a wide set of drinking patterns, and widely varying attitudes. It is a phenomenon likely to provide different information with different measurement approaches to even the same theoretical construct. Stages of Change is a variable of the Transtheoretical Model (Prochaska \& DiClemente, 1983) that attempts to separate students into meaningful subgroups and has been measured using two different approaches. Most research into the Stages of Change has used an algorithmic method of staging subjects in which the responses to a small set of items are used with a clear set of decision rules to assign subjects to stage (see Study I for the investigation of the algorithmic method). An alternative method is to use a multidimensional instrument that measures agreement with attitudes characterizing the Stages of Change. Cluster analysis is then used to group the subjects on the basis of their profiles across the scales. This analytic approach often results in a larger number of groups than found using the algorithmic method, and since the number of clusters is not predetermined, it is more exploratory. It also provides a greater amount of information about subjects by assessing their stage related attitudes on a number of scales.

It is thought that using a continuous measure has particular advantages in areas where there is not a consensus on criteria for healthy behavior. Alcohol consumption clearly is such a behavior. Experts argue as to what consumption patterns are harmful (see Peele, 1993) and certainly there are widely divergent views within a sample of college students. This is in contrast to a behavior like cigarette smoking, where most, if not all, agree that any regular smoking is a health hazard. With alcohol consumption there are health benefits and arguably social benefits that compete with the health and emotional detriments and risks. The 
continuous method of stage assessment can assess attitudinal sets consistent with stage membership with items that are less tied to specific behavioral criteria, whereas the algorithmic method depends on being able to determine and communicate criteria for healthy behavior.

The first scale staging instrument, called the University of Rhode Island Change Assessment (URICA), was initially developed as a generic instrument which asked subjects about their "problem", and was applied to psychotherapy clients (McConnaughy, DiClemente, Prochaska, \& Velicer, 1989; McConnaughy, Prochaska, \& Velicer, 1982). Others have constructed problem specific scales to measure these attitudes (Reed, 1993; Rollnick, Heather, Gold, \& Hall, 1992). The URICA has four scales labeled Precontemplation, Contemplation, Action, and Maintenance.

The set of cluster profiles found across varying behaviors has shown reasonable consistency (Blais \& Rossi, 1992). In addition to clusters for the five primary stages, clusters labeled Ambivalent, Immotive, and Uninvolved have been consistently found. Others have combined clusters that seemed to be stage subgroups resulting in five groups corresponding to the frve primary stages (Tsoh, 1993). This allows for a more direct comparison with the algorithmic method of stage classification.

There have been two studies to date that have investigated the Stages of Change for afcohol use using continuous URICA instruments. They have both provided strong validity evidence for the Stages of Change for the behavior of alcohol use. DiClemente and Hughes (1990) used the original URICA instrument with a population of out-patient alcoholic adults. The four scales had internal consistency coefficients of between .69 and .82 . Cluster analysis found five distinct groups, three of which closely matched the stages of Precontemplation, Contemplation, and Action. As expected in this population of drinking or very recently sober alcoholics, a Maintenance group was not found. The other two groups were characterized as Ambivalent and Uninvolved. The first of these groups can be thought of as between Precontemplation and Contemplation, and the second showed evidence of feeling defeated in 
their attempts to stop drinking. What is of interest about this study was the finding that less than $50 \%$ of the subjects were in the Contemplation or the Participation (Action like) subgroups despite being in treatment.

Rollnick et al (1992) also investigated Stages of Change using a URICA type scale instrument. They developed a three scale instrument specific to alcohol use using a population of heavy drinkers (a Maintenance scale was not applicable). They felt that the scale method was especially appropriate in an area with ambiguous criteria. They presented substantial data attesting to the validity of the scales, but unfortunately did not perform cluster analysis to fully investigate the population.

This study developed a multi-dimensional scale instrument to measure attitudes associated with Stage of Change for the process of cessation of immoderate alcohol use. This instrument, the University of Rhode Island Change Assessment for Alcohol (URICA-A), as opposed to the original URICA (McConnaughy et al., 1982), is specific to immoderate alcohol use. The resultant scales were used in a cluster analysis to examine what naturally occurring groups exist in relation to stage attitudes. These clusters were then compared to the stage groups found using the algorithmic method and then were combined in five groups corresponding to the five primary stages. External validity evidence is also presented. The use of the URICA-A promises to increase our understanding of the Stages of Change for the complex behavior of immoderate alcohol use by college students. 


\section{METHOD}

SUBJECTS

Subjects were 629 students surveyed at the University of Rhode Island in the fall of 1993 and spring of 1994 between the ages of 18 and 25 years and 11 months. Most of the subjects were recruited from undergraduate classes in the psychology department, although a variety of other classes were also sampled including physical education, pharmacology, nutrition, and sociology. A small percentage was recruited from the fraternities and the university health center.

The sample was $66.3 \%$ female, and $94 \%$ white. The distribution across the classes was $17.4 \%$ freshmen, $32.2 \%$ sophomores, $19.1 \%$ juniors, $26.2 \%$ seniors, and $5.3 \%$ either fifth-year or non-matriculated. The average age was 21.3 years. Of these students $47 \%$ were classified as in-state and $39 \%$ lived in dormitories, $10 \%$ lived in fraternities or sororities, and $51 \%$ lived off campus.

The use of alcohol in this sample was substantial. Using a criterion of at least one drinking occasion in the last 30 days, $92 \%$ were classified as active drinkers. These subjects drank on average 8.6 days a month and consumed on average 5.3 drinks per occasion. There were substantial differences in drinking by gender with drinking women averaging 7.3 days per month and 4.4 drinks per occasion. For men these figures were 11.0 days and 6.9 drinks. Drinking women reported an average maximum amount drunk on any occasion in the past month as 6.8 , and men as 12.1 .

\section{INSTRUMENTS}

The survey administered contained 282 questions, of which 5 item groups are of specific interest to this study. The full survey instrument is presented in Appendix A. 


\section{Demographic Assessment}

A set of 22 items asked about basic demographic information and drinking history. Variables examined in this study include age, gender, college class, living situation, number of days in the last month that alcohol was consumed (Days), the number of drinks consumed during a typical drinking occasion (Drinks), and the number of drinks consumed before subjects start to feel intoxicated (Intoxication).

\section{Stages of Change: Continuous_Measure}

The development of the University of Rhode Island Change Assessment for Alcohol instrument (URICA-A) followed the sequential rational method of scale development described by Jackson $(1970,1971)$ and elaborated on by Comrey (1988). An initial pool of 65 items were generated, as were stage definitions that described characteristic attitudes associated with each of the five stages that were hypothesized. Although previous research on the Stages of Cessation had only found four components representing the four stages of Precontemplation, Contemplation, Action, and Maintenance (DiClemente \& Hughes, 1990; McConnaughy et al., 1983), it was thought that as more recent research had clearly validated the stage of Preparation, an attempt was made to develop a scale to measure the attitudes of this stage. It was hypothesized that this scale would correlate highly with Contemplation.

Three expert judges categorized each item into one of five groups representing the five Stages of Change. Items that were judged by at least two judges to measure the hypothesized stage were reviewed. Of these a final set of 38 items was chosen as fully covering the five Stages of Change (7-8 items per stage). Subjects were asked to rate their agreement with the items on a 5-point likert scale ranging from (1) Strongly disagree to (5) Strongly Agree. 
Stages of Change: Algorithmic Assessment

The staging algorithm used answers from a branched set of five items to classify subjects into one of nine groups that represented the five Stages of Acquisition and the five Stages of Cessation with one group representing both the Maintenance stage of acquisition and the Precontemplation stage of cessation. The algorithm used was developed and investigated in Study I and used criteria of usually drinking four or more drinks at least once in a typical week for women and five or more drinks for men. The development and investigation of this staging method are presented in Study I.

\section{Alcohol Related Experiences}

Three scales, developed in Study I, that measure types of negative alcohol related experiences were included as a central validity variable. The scales are composed of 23 items developed from an original set of 31 items and are labeled Excess, Distress, and Problem. The three scales were developed using principal component analysis with oblique rotation and have moderate inter-scale correlations ranging from .37 to .44 and high internal consistencies as measured by Cronbach's Alpha ranging from .80 to .88 . The Excess scale is hypothesized to measure problems associated with short-term excessive or binge drinking. The Distress scale measures experiences of emotional or introspective distress, and the Problem scale measures consequences of alcohol consumption that are associated with long term problem drinking.

Subjects were asked to circle the number of times in the last 12 months that they had had each experience related to their alcohol consumption. The following 6-point response scale was provided: $0,1,2,3-5,6-9,10$ or more times.

\section{Measures of Psychosocial Development}

Three scales measuring aspects of psychosocial development were included in a 32 item set of questions. These scales are subscales of the Student Development Task and 
Lifestyle Inventory (SDTLI), which has received intensive development over the last 15 years (Winston \& Miller, 1987). The scales were based on the work of Chickering (1969).

The first subscale, Peer Relationships, measures the subjects' ability to develop relationships that have greater openness, trust, and independence, to resist pressure to conform, and to accept differences. The second scale, Emotional Autonomy, measures the ability of subjects to be free from the need for continuous reassurance, to be prudent in risk taking, to have confidence in their decision making, and to voice dissenting opinions. The third scale, Lifestyle Planning, measures the establishment of a personal direction in one's life that incorporates values, family plans, and vocational objectives. Subjects who score high on this subscale have the capacity to follow through on commitments and can specify how current activities relate to their goals.

In an attempt to improve the reliability of these scales the item fomat was changed from a True/False to a 5-point Likert scale varying from $I=$ Strongly disagree to $5=$ Strongly agree. This response format is generally deemed to produce superior psychometric properties (Comrey, 1988). Internal consistency as measured by Cronbach's coefficient alpha was .68 for Peer Relationships, .59 for Emotional Autonomy, and .76 for Lifestyle Planning.

\section{PROCEDURE}

The survey for this study was included within a larger survey that assessed additional aspects of drinking behaviors and attitudes. Informed consent was obtained and subjects were assured of the confidentiality of their responses (See Appendix B for a copy of the consent form). Surveys were completed during class time, or subjects took the survey home and returned it. Most, although not all, of those doing the survey on their own time received a small amount of class credit for returning the survey. A few students who completed the survey at the health services center were given a pen emblazoned with the university name in exchange for completing the survey. 


\section{RESULTS}

\section{DeVelopment of a SCALE MEASURe}

\section{Exploratory Component Analysis}

Cases which had missing data on more than three of the 38 URICA-A items were eliminated from the analysis leaving $98 \%$ of the sample $(\underline{N}=616)$. Eliminating subjects in the Stages of Acquisition was considered and rejected as the URICA-A is envisioned as an instrument useful in staging students independent of algorithmic stages. The sample was then randomly divided into two subsamples. An exploratory component analysis was conducted on the 38 by 38 matrix of item intercorrelations generated from Sample 1 using pair-wise deletion $(\underline{\mathrm{N}}=294$ ). The number of components to retain was determined by comparing the results of three procedures that have been shown to be valid predictors of the correct dimensionality of an item set (Zwick \& Velicer, 1986). The scree procedure (Catell, 1966) suggested two or five components; minimum average partial procedure (Velicer, 1976), and parallel analysis (Horn, 1968; Lautenschlager, 1989) indicated four components. Oblique rotations extracting both four and five component solutions were performed and evaluated. The fifth component accounted for only $3.7 \%$ of the unrotated variance and was composed of only two items. The four factor solution was chosen for interpretation and further analysis.

A number of items loaded complexly on two of the components. Complete elimination of these complex items would have left one component with only three high loading items. After examination of component content, it was decided to delete this component by deleting the three items that uniquely defined this minor component. In future research, additional items may be generated which could result in a well identified component. In the present analysis, the component was too poorly defined and tentative for inclusion.

The final solution consisted of 21 items and three components, interpreted as representing three stage related attitudes. The results of this analysis were interpreted as three 
scales measuring aspects of the Stages of Change. The first component, Precontemplation was composed of four Precontemplation items which all suggested acceptance of heavy drinking. Its internal consistency as measured by Cronbach's Coefficient Alpha for Sample 1 was .71.

The second component was composed of 12 items including six which were hypothesized to measure Contemplation, five hypothesized Preparation items, and one Action item. The Action item could be interpreted as consistent with the Contemplation and Preparation items and was not eliminated from the exploratory analysis. This component, labeled Contemplation, had an alpha of .86 for sample 1 .

The third component, labeled Maintenance, was composed of four Maintenance items and one Action item. All these items suggested a past reduction in drinking. This component had a coefficient alpha of .79. These three components explained $47.7 \%$ of the unrotated variance of the reduced set of 21 items. Table 3-1 presents the final item set with component loadings.

\section{Confirmatory Factor Analysis}

Confirmatory factor analysis using EQS structural modeling program (Bentler, 1989) was performed using the hold out sample $(\mathrm{N}=322)$. Three models were run. The null model which posits 21 independent variables is not expected to fit the data but generates a set of statistics with which the other models can be compared. The second model was a three uncorrelated factors model and the third was a three correlated factors model.

A set of fit indices including Chi square statistic, Comparative Fit Index (CFI), NonNormed Fit Index (NNFI), Root Mean Square (RMS), and Incremental Fit Index, type 2 (IFI2) were calculated to assess model fit. Since these indices all produced a similar pattern of results, only the IFI2 is reported here. These fit indices indicated that the correlated three factor model was superior to the others. However, model fit was less than ideal (IFI2 $=.87$ ) 
suggesting that model improvement was possible. A step-wise model modification procedure was undertaken using the correlated-factor model.

A number of considerations were assessed in making model modifications, including factor loadings, residuals, and the LM statistic (Bentler, 1989). The LM statistic indicates the amount of model improvement that would occur if a non-estimated path were allowed to be non-zero. This can be used as an indicator of item complexity. Because of the low number of items in the Precontemplation and Maintenance scales, items from these scales were preserved if possible. Since it is not fully predictable how the model parameters will react to model modifications, only one change was made at a time, and then the model was reassessed.

This procedure resulted in the removal of four items from the Contemplation scale and substantial model fit improvement (IFI2 =90). Figure $3-1$ presents final item set, loadings, and correlations of the structural model. Scale scores were calculated by taking the unweighted mean of items for subjects who answered at least $50 \%$ of the items of the scale. Table 3-2 provides means, standard deviations, and coefficient alphas of the final scales for both samples.

\section{Cluster Analysis}

In order to determine whether the URICA-A scales are useful in classifying subjects into cohesive and meaningful subgroups a cluster analysis was performed. This approach has been used in a number of previous studies for the behaviors of smoking acquisition (Stern, Prochaska, Velicer, \& Elder, 1987), exercise (Reed, 1993) and alcohol use (DiClemente \& Hughes, 1990). Comparison to previous results will assist in the interpretation of cluster solutions.

Since the relationship between these scales and the algorithmic stages are one of the main focuses of this investigation, the sample was limited to those who are presently or have previously been drinking above criterion for a sample of 454 subjects. (This does include 
those in the Action for Acquisition stage $(\mathrm{N}=35)$ ). Scale scores were converted into $\mathrm{T}$-scores $(\underline{M}=50, \underline{S D}=10)$ to equate the weight of each scale score in the cluster analysis.

Ward's method of agglomerative hierarchical clustering was used on a Euclidian distance matrix for the 454 subjects. This method has been demonstrated to have performed better than other methods of clustering (Milligan, 1980; Milligan \& Cooper, 1987). There is no highly reliable and agreed upon statistical method for determining the correct number of clusters to retain. In this study two statistical indices were assessed, the Cubic Clustering Criterion (Searle, 1983) and the Pseudo F-Test (Calinski and Harabasz, 1974), but the heaviest weight in deciding on a cluster solution was given to the interpretability of the cluster profiles. Given the consistency of the findings across a number of behaviors, this method has a sound theoretical basis (see Blais \& Rossi, 1992).

The two statistical indices of number of clusters to interpret did not clearly indicate a solution, but both suggested that the correct solution was in the seven to ten cluster range. Solutions providing three to twelve clusters were created and interpreted. The seven or eight cluster solutions proved to be most interpretable. Although the eight cluster solution included two variations of a cluster found to be unitary in earlier work, these variations were thought to be important. The additional clusters identified in the nine to twelve cluster solutions proved to be minor variations of the clusters they split off from. The eight cluster solution was chosen for further analysis.

Profiles for the eight clusters are presented graphically in Figures 3-2 to 3-5 and are described below.

Precontemplation-1 $(\mathrm{N}=50)$ - Subjects classified into this cluster are characterized by above average endorsement of the Precontemplation scale, and below average endorsement of the Contemplation and Maintenance scales. These subjects feel their heavy drinking is acceptable and are not considering changing it. 
$\underline{\text { Precontemplation-2 }}(\underline{N}=115)$ - Subjects classified into this cluster are characterized by above average endorsement of the Precontemplation scale, average endorsement of the Contemplation scale, and somewhat below average endorsement of the Maintenance scales. Although these subjects feel that their heavy drinking is acceptable they are more willing to consider the need to change it than those in Precontemplation-1.

$\underline{\text { Immotive-PC }}(\underline{\mathrm{N}}=51)$ - There were 2 immotive clusters, so called because they scored highest on the scale for the two stable stages, Precontemplation and Maintenance. Subjects classified into this first cluster possess substantially above average scores on Precontemplation, substantially below average scores on Contemplation, and average scores on Maintenance. These subjects both feel that their heavy drinking is quite acceptable, but that they are fairly confident that a previous reduction in their drinking makes their drinking patterns acceptable. Given these attitudes it is not surprising they have the lowest score on the Contemplation scale of all the clusters, with no intention to reduce their drinking. It is hypothesized that these subjects might be similar to subjects found for the behaviors of low-fat diets, exercise, and safe-sex who were labeled Pseudo-Maintainers (Redding, 1993; Reed, 1993; Rossi, 1993).

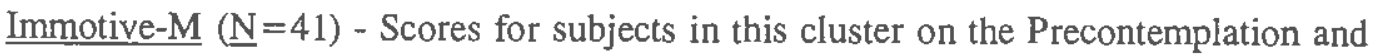
Maintenance scales were reversed from the previous cluster, with Maintenance being above average and Precontemplation about average. Similar to the Immotive-PC cluster, the Contemplation score was below average. These subjects think that they have no reason to change their behavior believing they have already done so, and show moderate acceptance of their own heavy drinking.

Contemplation $(\underline{N}=68)$ - Subjects classified into this cluster are characterized by above average endorsement of the Contemplation scale, and below average endorsement of the 
Precontemplation and Maintenance scales. These subjects feel their drinking habits need to change.

Preparation $(\underline{N}=48)$ - Subjects classified into this cluster are characterized by substantially above average endorsement of the Contemplation scale, and average endorsement of the Precontemplation and Maintenance scales. As can be seen in Figure 3-4 the profiles for the Contemplation and Preparation clusters have the same shape but differ in height. Because of the higher Maintenance and Contemplation scores this group is hypothesized to be a Preparation cluster.

Action $(\underline{N}=54)$ - Subjects in this stage had well above average scores on the Maintenance scale, above average scores on the Contemplation scale, and substantially below average scores on the Precontemplation scale. These subjects think they have made substantial changes but need to make further reductions in their drinking.

Maintenance $(\underline{N}=27)$ - Subjects in this stage had well above average scores on the Maintenance scale (equal to the Action cluster), below average scores on the Contemplation scale, and the lowest scores of any cluster on the Precontemplation scale. These subjects think they have made substantial changes and do not feel they need to make further reductions in their drinking. Furthermore they have a very negative view of heavy drinking behaviors.

\section{RELATIONSHIP BETWEEN ALGORITHMIC STAGES AND URICA-A CLUSTERS}

\section{Cross-classification}

To investigate the relationship of the URICA-A to the discrete staging algorithm two analyses were conducted. The first is an investigation of the cross-classification between the algorithmic stages and the clusters described above. The second is a discriminant function analysis with the URICA-A scales as predictors of stage membership. 
Table 3-3 presents the percentages of each cluster as classified into each stage. For simplicity, subjects in Action for Acquisition were combined with those in Precontemplation. The distributions of these stages across the clusters were very similar. By examining these data agreement can be gauged and the disagreements described both quantitatively and qualitatively. For clarity, percentages greater than $20 \%$ are in bold and the greatest row percentage is underlined.

Both the Precontemplation-1 and Precontemplation-2 cluster had similar crossclassification patterns with a large majority of cases in Precontemplation (86\% and $82 \%$ respectively) and most misclassifications in Contemplation ( $8 \%$ and $10 \%$ ). This pattern supports the interpretation of these clusters as representing types of Precontemplators, but does not give any clear indication as to the differences between these groups.

The majority of the Immotive-PC cluster was also classified as Precontemplators by the discrete algorithm (73\%). What differs with this group is that the majority of misclassified cases are in Maintenance $(12 \%)$. The pattern for Immotive-M was quite different with only $24 \%$ in Precontemplation, and the majority classified as either in Action (27\%) or Maintenance (39\%). This result suggests that this group is not a type of Precontemplation but a late stage group. It also supports the use of an eight-cluster solution, as these two Immotive groups were the last to separate.

The majority of subjects in the Contemplation cluster were classified as Precontemplators by the algorithm (57\%), with the second largest group classified as Contemplation (19\%). This suggests that either this cluster is an advanced type of Precontemplation, or that the algorithm over-includes subjects into Precontemplation, by far the largest algorithmic stage.

The Preparation cluster was fairly evenly spread across the stages of Precontemplation, Contemplation, and Preparation (35\%, 27\%, and $21 \%$ respectively). Although this group is more advanced on average than the Contemplation cluster its characteristics are still unclear. 
The Action cluster's largest stage classification was into Maintenance $(47 \%)$, with substantial amounts in Precontemplation (22\%) and Action (13\%). This is clearly a more advanced cluster, lending some support to its interpretation as an Action cluster, especially when compared to the last cluster.

A majority of subjects classified into the Maintenance cluster were also classified into the Maintenance stage (67\%), with most misclassification happening in the Action stage (15\%) supporting the interpretation of this clusters.

\section{Discriminant Function Analysis}

Discriminant function analysis was undertaken to further investigate the relationship between the URICA-A scales and the algorithmic stages, and in particular to assess the ability of the URICA-A instrument to predict algorithmic stage membership. Subjects classified into the algorithmic Stages of Cessation were used. Six cases were removed because of missing data leaving a final sample size of 420 .

Three discriminant functions were calculated with a significant Wilk's Lambda $(\Lambda=.51)$ and combined $\chi^{2}(12)=278.32, \mathrm{Q}<.0001$. The first function had a canonical correlation of .64 and explained $83 \%$ of the variance. After removal of the first function there was still significant discriminating power with Wilk's Lambda $(\Lambda=.87)$ and $\chi^{2}(6)=55.80$, $\mathrm{p}<.0001$. The second function had a canonical correlation of .35 and accounted for $16 \%$ of the variance. After the removal of the second function the third function did not reach significance.

An ordered structure matrix of the pooled within-groups correlations between the discriminant variables and the discriminant function is presented in Table 3-4. Using a criterion of $\geq .30$ as a cutoff for interpretation, loadings suggest that Function 1 has a strong positive association with the Maintenance scale and a moderate negative association with the Precontemplation scale. This function measures attitudes consistent with the giving up and disapproval of heavy drinking. The second function is very strongly associated with the 
Contemplation scale score, and has a small negative association with the Precontemplation score. It represents the intention to change disapproved heavy drinking.

Group centroids are plotted in Figure 3-6. As can be seen all five stages are ordered and separated by Function 1 with the largest separation occurring between Action and Maintenance. Function 2 separates stages that are not considering future behavior change (Precontemplation, Action, and Maintenance) from those that are planning change (Contemplation and Preparation). Together these two discriminant functions clearly separate the five groups.

A jackknifed classification procedure was used to classify the subjects used in this analysis into one of the five stages of change, resulting in 213 or $50.7 \%$ of the subjects being correctly classified into their algorithmic stage. This is approximately two and a half times the $20 \%$ who would be correctly classified by chance alone. Cross-classification results are presented in Table 3-5. Most misclassified subjects were placed into adjacent stages. An exception to this pattern was that $24 \%$ of those in Action were classified into Precontemplation.

\section{DEVELoPMENT OF ADJUSTED Clusters}

To conceptualize the clusters as either Stages of Change or subgroups of these stages allows for two types of analysis. One is when each cluster is analyzed as a separate and meaningful group. The other is to combine subtypes into five groups representing the five primary stages of change. The latter approach simplifies the analysis and allows for the comparison of results with other research using the five primary stages (see Tsoh, 1993 for an example of this approach). This approach will be used in assessing the external validity of the clusters.

The set of clusters was altered in three ways. First the three clusters, Precontemplation-1, Precontemplation-2, and Immotive-PC were combined into a larger Precontemplation cluster. This left six clusters, five of which were confidently interpreted as 
relating to the five Stages of Cessation. The sixth group, the Immotive-M cluster, was not clearly related to a primary stage and was not included in further statistical analysis although it was included in figures for comparisons to the other groups. This reduced the clusters to a set directiy comparable to the algorithmic Stages of Change allowing for judgments as to which is a superior method of classifying subjects. A final adjustment was made by eliminating subjects whose stage as determined be the algorithmic measure was more than one stage away from the presumed cluster stage. For example 21 of the 216 subjects in the combined Precontemplation cluster who were staged algorithmically into Preparation, Action, or Maintenance were removed. This created groups which were less likely to contain significant numbers of misclassifications. A total of 76 of 413 subjects (18.4\%) were eliminated in this process, including a low of $9.7 \%$ of the three Precontemplation clusters to a high of $43.8 \%$ of the Preparation cluster. These final groups will be called Adjusted Clusters.

\section{EXTERNAL VALIDITY}

Validating variables used in this study can be conceptualized into four categories: demographic variables; alcohol consumption variables, negative consequences of drinking as measured by the Alcohol-Related Experiences and psychosocial development variables. Differences among Adjusted Clusters on these variables provide validation of their usefulness in investigating immoderate alcohol use and help characterize the clusters. Three of the demographic variables are categorical and are analyzed using the chi-square statistic. The remaining validity variables are continuous and are analyzed using analysis of variance techniques.

Demographics. The demographic variables included gender, living situation, class, and age. Results show that Stage of Cessation as measured by the Adjusted Clusters and gender were significantly related $\left(\chi^{2}(4)=16.72, \mathrm{Q}<.01\right)$. Women were under-represented in 
Precontemplation and over-represented in Action and Maintenance, suggesting that a higher proportion of women who had had a period of immoderate drinking had reduced their drinking to moderate levels. Distributions of subjects by Adjusted Cluster across the three categorical variables are presented in Table 3-6.

The Adjusted Clusters were not significantly related to either living situation or class. As a follow-up, students were separated into underclassmen (freshmen and sophomores) and upper classmen (juniors and seniors). Chi square analysis revealed an association at the $\mathrm{p}<.10$ level $\left(\chi^{2}(4)=8.50, \mathrm{p}<.10\right)$. The underclassmen were over-represented in Precontemplation and under-represented in Preparation, Action, and Maintenance suggesting that upperclassmen are more advanced in the Stages of Cessation for immoderate drinking.

A significant relationship between Adjusted Clusters membership and subject age was found $\left(\underline{F}(4,330)=7.10, \underline{\mathrm{p}}<.0001, \omega^{2}=.07\right)$, and follow-up Tukey tests showed that students in the Action cluster were older than those in the Precontemplation or Contemplation clusters and those in Maintenance were older than those in Precontemplation. The age to stage relationship is presented graphically in Figure 3-7. As can be seen, there is a clear relationship between age and stage, with about a year increase in age between the first two stages and the last two, with the Preparation cluster in between. The Immotive-M cluster is closest in age to the Maintenance group. See Table 3-7 for means and standard deviations by Adjusted Cluster for age and all other continuous variables reported in this section.

Alcohol consumption variables. The Adjusted Clusters showed a strong relationship to the alcohol consumption variables. The result of the MANOVA was $(\Lambda(12,862.8)=.70$ $\mathrm{Q}<.0001$ ) indicating that the stage variable accounts for $30 \%$ of the variance. All three follow-up ANOVA's were significant (Days: $\left(\underline{F}(4,331)=24.02, \underline{\mathrm{p}}<.0001, \omega^{2}=.22\right)$; Drinks: $\left(\underline{F}(4,331)=23.50, \underline{\mathrm{g}}<.0001, \omega^{2}=.21\right)$; Intox: $\left.\left(\underline{\mathrm{F}}(4,330)=8.96, \underline{\mathrm{p}}<.0001, \omega^{2}=.09\right)\right)$. Followup Tukey tests on these three variables showed that subjects in Precontemplation, Contemplation, and Preparation drank on significantly more days in the last month, consumed 
more drinks, and felt that it took more alcohol before they started to feel intoxicated than those in Action and Maintenance. Means by Adjusted Cluster are presented in Figure 3-8. As can be seen in this figure, there is a threefold decrease in both the number of drinks and the frequency of drinking occasions between Precontemplation and Maintenance. Moreover, those in Precontemplation and Contemplation had nearly identical values on all three of these variables. Those in Preparation drank on fewer days, but consumed equal amounts per occasion suggesting frequency of immoderate drinking might be the first aspect that is modified. As would be expected for these consumption related variables, the largest change occurred between the Preparation and Action stages, the behavioral transition in stage progression. Those in Maintenance drank as often but consumed less than those in Action although this difference was not significant. Another pattern of interest is that there was a crossover between the Intoxication and Drinks variables between Preparation and Action, indicating that on average those in the first three stages were drinking enough to begin to feel intoxicated, whereas those in the last two stages drank less than this amount. The ImmotiveM cluster scored between the Preparation and Action stages on all three of these variables.

Alcohol-Related Experiences. The relationship between Stage of Cessation as measured by the Adjusted Clusters and the three Alcohol-Related Experiences scales proved to be significant $(\Lambda(12,860.2)=.70, \mathrm{p}<.0001)$ accounting for $30 \%$ of the variance. All three follow-up ANOVA's were significant (Excess $\left(\underline{F}(4,327)=20.65, \mathrm{p}<.0001, \omega^{2}=.19\right)$; Distress: $\left(\underline{\mathrm{F}}(4,327)=11.58, \mathrm{q}<.0001, \omega^{2}=.11\right)$; Problem: $(\underline{F}(4,327)=4.43, \mathrm{p}<.01$, $\left.\omega^{2}=.04\right)$ ). T-scores by stage are presented graphically in Figure 3-9. Follow-up Tukey test showed that subjects in Precontemplation, Contemplation, or Preparation had higher scores on the Excess scale than those in Action and Maintenance. On the Distress scale those in Contemplation and Preparation scored higher than those in Precontemplation, Action, or Maintenance. Also those in Precontemplation scored higher than those in Maintenance. There were fewer significant pair-wise differences on the Problem scale, and this may be a 
result of its restricted range. Subjects in Contemplation scored significantly higher than those in Action or Maintenance and those in Preparation scored higher than those in Maintenance. The rise in scores from Precontemplation to Contemplation and Preparation on the Distress and Problem scales is of interest. It is unclear whether this curvilinear pattern is because those who are considering or planning change do so because they can not "handle their liquor" and experience more negative sequelae of their drinking or that they are more willing to recognize their negative alcohol-related experiences, referred to in the literature on alcoholism treatment as the breakdown of denial. Support for the latter hypothesis can be seen in the fact that this curvilinear pattern was most pronounced on the Distress scale, which is the most subjective of the three scales, and that this pattern was not substantial on the Excess scale, which is the most concrete and least socially disapproved. The Immotive-M cluster scored at about the level of Maintainers for both the Problem and Distress scales, but between Preparation and Action on the Excess scale.

Psychosocial development scales. The MANOVA conducted on the three developmental scales was also significant $(\Lambda(12,854.9)=.93, \mathrm{p}<.05)$, but explained only $7 \%$ of the variance. Follow-up ANOVA's were not significant for either the Peer Relationship or Emotional Autonomy scales but was for the Life Planning scale $(\mathbf{F}(4,325)=5.24, \underline{\mathrm{p}}<.001$, $\omega^{2}=.05$ ). Follow-up Tukey tests only suggest that those in Action have a more developed life plan than Precontemplators, Contemplators, or Preparers. T-scores by stage are presented in Figure 3-10. This significant result might be explained by the older age of those in Action. Alternatively it might reflect a general underlying attitude of initiating positive changes and planning for the future. The lack of clear differences on the other scales, and their nearly flat trends suggests that there is not a clear relationship between Stage of Cessation and psychosocial development as hypothesized. Interestingly, although the Immotive-M cluster scored about the same as Maintainers on the Life Planning scale, its scores on the other two scales were substantially lower than any other group, suggesting this group, despite being 
somewhat future focused, is more dependent on others emotionally and less capable of resisting peer-pressure. 


\section{DISCUSSION}

This study developed a scale instrument, the URICA-A, that demonstrated usefulness in classifying college students into groups representing the Stages of Change for the cessation of immoderate alcohol use. Differences among these stage groups on a number of variables conformed to expectations and supported the use of the URICA-A and the Transtheoretical Model in understanding this behavior. Aspects of this study are discussed in more detail below.

\section{THE URICA-A}

The URICA-A in its final form performed well in generating a set of clusters useful in investigating immoderate alcohol consumption. An attempt was made in its development to measure five attitudinal sets, one for each of the hypothesized Stages of Change. This had not been successfully done in previous research, but this ambitious goal was attempted because of the increasing confidence in the Preparation stage as well as a clearer understanding of its attributes. Unfortunately only three dimensions were measured, Precontemplation, Contemplation, and Maintenance.

An Action subscale has been consistently found in other areas of research on the cessation of behaviors including alcohol use (DiClemente \& Hughes, 1990; Rollnick et al., 1992). There are two explanations of this finding. First, the original item set might not have adequately tapped this dimension. Some traditional items were not used because of the effort to measure a Preparation scale and restrictions on the number of items that could be included. Adding these, and perhaps other new items might result in a well identified component.

Second, is the possibility that this attitudinal dimension does not exist for this population and behavior, but that those in Action possess a distinctive combination of attitudes. Results from the cluster analysis suggest that this is a combination of disapproval of heavy drinking, a commitment to reduce their present drinking, along with a history of 
heavier drinking that they feel they have stopped. Also, for this behavior meaningful change entails reducing, not eliminating alcohol consumption. There is no clear behavioral marker to define action, or around which attitudes can coalesce.

The lack of separate Contemplation and Preparation subscales is less surprising. Preparation is often thought of as a sub-stage of Contemplation with mostly overlapping attitudes. The items created to measure Preparation attempted to assess the attitudes and behaviors that characterize this stage such as the solidification of commitment to change, active and concrete planning, and the beginning of behavior change. These items largely loaded with the more traditional Contemplation items, with about half the final scale items being originally hypothesized to be Preparation items. Given the nature of the stages, principal component analysis might not be the best method for developing these scales, but a hierarchical structural model, with the Contemplation and Preparation latent variables loading on a second order latent variable might better capture the stage dimensionality. Furthermore it might be difficult to measure such a stage within a population that has very low numbers of Preparers (3.4\% in this sample). Efforts to further develop the URICA-A should focus on developing an Action scale as the first priority, improving the psychometrics of the Precontemplation scale as a second, and possibly including an additional effort to develop a separate Preparation scale.

\section{The Clusters AND ADJusted Clusters}

The URICA-A was used in a cluster analysis to assess the possible usefulness of this analytic approach. The results suggest that this is a very promising route of investigation. In the present study eight interpretable clusters were found, demonstrating a more complex classification than was produced with the algorithmic staging method. Whether subjects are more reliably classified and whether these added group distinctions are predictive, useful for model building or intervention design, awaits further research. 
The relation of each cluster to the Stages of Change can be examined by both the cluster scale profile and the cross-classification with the algorithmic stages. These results supported the existence of three types of Precontemplators labeled Precontemplation-1, Precontemplation-2, and Immotive-PC. These clusters had nearly identical distributions across the algorithmic stages, but their scores differed on the Contemplation and Maintenance URICA-A scales. Subtypes within stages have been demonstrated using measures of Decisional Balance and Temptation (Velicer, Hughes, Fava, Prochaska, \& DiClemente, in press) and are reasonable to expect. Further research into the differences between these groups, especially since Precontemplators comprise the largest group, would be worthwhile.

The cross-classification with algorithmic stages clearly supports the labeling of the Maintenance cluster, and provides support, although somewhat less strongly, for the naming of the Contemplation, Preparation, and Action clusters. The nature of the last cluster, Immotive-M, is more in question. This cluster is of interest because clusters having similar profiles have not been found in previous research. This cluster separated last in the cluster analysis from the other Immotive cluster, yet has a very different cross-classification pattern with the algorithmic Stages of Change. This cross-classification pattern suggests that this cluster is more closely related to the Action or Maintenance stages. It is possible that this is a group of Pseudo-Maintainers, as found in other behavioral domains (Redding, 1993; Reed, 1993). This view is supported by the fact that they are drinking less than average, but still more than those in Action or Maintenance, and that they are still experiencing significant amounts of negative consequences of their drinking.

An additional hypothesis is that members of the Immotive-M cluster reduced their drinking without much intention, difficulty, or conscious effort and haves not developed a negative view of heavy drinking. It is likely that for many college students, drinking patterns are heavily influence by environmental variables, especially interpersonal ones. This hypothesis is supported by findings in Study III that show that members of this cluster rate the Cons of alcohol consumption as low as Precontemplators and much lower than late stage 
subjects. Furthermore their low scores on the Peer Relationships and Emotional Autonomy scales suggest that this group is strongly affected by their interpersonal environment. Full understanding of this cluster awaits further research.

The results of this study also clearly support the validity of the Adjusted Clusters as a method of measuring Stages of Change for the cessation of immoderate alcohol use. The strongest result found in this study is the clear relationship of the Adjusted Clusters to the Alcohol-Related Experiences scales. These resuits support a clear relation between stage membership and the negative sequelae of drinking. If these results hold up in longitudinal studies, moving from early stages to Action or Maintenance clearly reduces ones risk of being harmed by alcohol consumption. For the majority of college students it is short term risks that are most problematic, although the results of the Problem scale also suggest that for some students a reduction in risk associated with chronic use is also achieved.

The difference between a cluster analytic approach and an algorithmic approach to classification of subjects is in the relative importance given to attitudes and behaviors.

Whereas items of the staging algorithm assess either behavior or intentions linked to a specific behavior, the items of a URICA instrument assess attitudes more loosely associated with behavior. The divergence of attitudes and behavior is an important area of investigation and probably varies across behaviors. One could hypothesize that the more clearly a behavioral criterion is understood and accepted by the study population, the fewer discrepancies there will be between clusters and algorithmic stages. For example, in areas like exercise (Reed, 1993), safe-sex (Redding, 1993), and low-fat diets (Rossi, 1993) a consistent finding is of a Pseudo-maintainer group. The Pseudo-maintainer feels he is maintaining satisfactory behavior change while he is actually still behaving in unhealthy ways. It is unlikely such a group would be found for smoking, where abstinence is clearly recognized as the only healthy option. From a health perspective Precontemplators and Pseudo-maintainers are alike - both need to change their behavior (although the behavior of Pseudo-maintainers is probably closer 
to healthy standards). In contrast, in designing interventions, the attitudinal differences of these two groups would likely be important to consider.

The Adjusted Clusters can be compared to the algorithmic Stages using external validity evidence. On the consumption variables, the algorithmic Stages explain a somewhat greater proportion of the variance as measured by the MANOVA ( $36 \%$ to $30 \%$ ) or by $\omega^{2}$ for each variable. The largest difference was on the Intoxication variable (17\% to $9 \%$ ). This is not a surprising result considering that the items for the algorithmic staging method asked explicitly about consumption. Both measures of stage explain similar amounts of variance on the Alcohol-Related Experiences scales (algorithmic Stages: 28\%, Adjusted Clusters: 30\%). The largest differences in the pattern of means by stage for these variables suggest that the Preparation Adjusted Cluster drinks slightly more, but on few days and experiences somewhat higher levels of negative consequences than the same algorithmic Stage. Also the Action and Maintenance Adjusted Cluster are lighter drinkers and the Maintenance Adjusted Cluster experiences fewer negative consequences.

Overall this comparison does not suggest a clear superiority of one method of measurement over the other. If the Adjusted Chusters are to outperform the algorithmic Stages it will be on other measures more closely linked to attitudes about drinking. These differences, although not outcome variables, might be more important to the change process and in designing effective interventions. The Transtheoretical Model of Change provides a number of variables that have been shown to be important in facilitating behavior change. Study III compares these staging methods for the Decisional Balance construct.

In summary, the preceding analysis explored the development of the URICA-A and its use in classifying subjects in Stages of Change as delineated by the Transtheoretical Model of Change. Results suggest that this method of subject classification is valid and useful in investigating the behavior of immoderate alcohol use. This method shows special promise for a behavior where there are not clear behavioral criteria for healthy choices. 
The eight cluster solution, although not investigated in depth in this study, suggests that there might be meaningful distinctions between subjects not captured by the Stage of Change construct. This has been demonstrated in other research (DiClemente \& Hughes, 1990; Velicer et al., in press). The important question of whether these groups have predictive utility is unclear and awaits further research.

The cluster analytic method has the disadvantage of being a more complicated and costly method than the algorithmic method in terms of length of the instrument, the extensive analysis, and the sample size required. Furthermore, the results obtained in this project suggest that additional development needs to be done on the URICA-A scales which are the basis of the clusters. Specifically, further developmental work aimed at increasing the reliability of the Precontemplation scale and at measuring the attitudes associated with Action, is envisioned. 


\section{REFERENCES}

Bentler, P. M. (1989). EQS:Structural equations program manual. Los Angeles, California: BMDP Statistical Software.

Blais, L. M. \& Rossi, J. S. (1992). Stages of change: Comparing clusters across four health behaviors. Paper submitted to the 100th convention of the American Psychological Association in Washington, DC.

Calinski, T. \& Harabasz, J. (1974). A dendrite method for cluster analysis. Communications in Statistics, 3 , 1-27.

Cattell, R. B. (1966). The Scree test for the number of factors. Multivariate Behavioral Research, 1, 245-276.

Chickering, A. W. (1969). Education and identity. San Francisco: Josset-Bass.

Comrey, A. L. (1988). Factor-analytic methods of scale development in personality and clinical psychology. Journal of Consulting and Clinical Psychology, 56, 754-761.

DiClemente, C. C. \& Hughes, S. O. (1990). Stages of change profiles in outpatient alcoholism treatment. Journal of Substance Abuse, $2,217-235$.

Horn, J. L. (1965). A rationale and test for the number of factors in factor analysis. Psychometrika, 30, 179-185.

Jackson, D. N. (1970). A sequential system for personality scale development. In Spielberger (Ed.), Current topics in clinical and community psychology (Vol. 2, pp. 61-96). New York: Academic Press.

Jackson, D. N. (1971). The dynamics of structured personality tests: 1971. Psychological Review, 78, 229-248.

Lautenschlager, G. J. (1989). A comparison of alternatives to conducting Monte Carlo analyses for determining parallel analysis criteria. Multivariate Behavioral Research, 24, 365-395. 
McConnaughy, E. A., DiClemente, C. C., Prochaska, J. O., \& Velicer, W. F. (1989).

Stages of change in psychotherapy: A follow-up report. Psychotherapy, 26, 494-503.

McConnaughy, E. A., Prochaska, J. O., \& Velicer, W. F. (1982). Stages of change in

psychotherapy: Measurement and sample profiles. Psychotherapy: Theory, Research and Practice, 20 , 368-375.

Milligan, G. W. (1980). An examination of the effect of six types of error perturbation on fifteen clustering algorithms. Psychometrika, $\underline{45}, 325-342$.

Milligan, G. W. \& Cooper, M. C. (1985). An examination of procedures for determining the number of clusters in a data set. Psychometrika, 50, 159-179.

Peele, S. (1993). The conflict between public health goals and the temperance mentality. American Joumal of Public Health, $83,805-810$.

Prochaska, J. O. \& DiClemente, C. C. (1983). Stages and processes of self-change of smoking: Toward an integrative model of change. Journal of Consulting and Clinical Psychology, 51, 390-395.

Redding, C. A. (1993). The transtheoretical model applied to safer sex behavior among university students: A cross-sectional investigation. Unpublished doctoral dissertation, University of Rhode Island, Kingston, RI.

Reed, G. R. (1993). The transtheoretical model and exercise behavior: A comparison of five staging methods. Unpublished master's thesis, University of Rhode Island, Kingston, RI.

Rollnick, S., Heather, N., Gold, R., \& Hall, W. (1992). Development of a short 'readiness to change' questionnaire for use in brief, opportunistic interventions among excessive drinkers. British Journal of the Addictions, $87,743-754$.

Rossi, S. R. (1993). Application of the Transtheoretical Model of behavior change to dietary fat reduction in a naturalistic population. Unpublished doctoral dissertation, University of Rhode Island, Kingston, RI. 
Searle, W. S. (1983). Cubic clustering criterion (Tech. Rep. A-108). Cary, NC: SAS Institute.

Stern, R. A., Prochaska, J. O., Velicer, W. F., \& Elder, J. P. (1987). Stages of adolescent cigarette smoking acquisition: Measurement and sample profiles. Addictive Behaviors, $\underline{12}, 319-329$.

Tsoh, J. Y. (1993). Motivation and stages of change among drug addicts in drug abuse treatment programs. Unpublished master's thesis, University of Rhode Island, Kingston, RI.

Velicer, W. F. (1976). Determining the number of components from the matrix of partial correlations. Psychometrika, 41, 321-327.

Velicer, W. F., Hughes, S. L., Fava, J. L., Prochaska, J. O., \& DiClemente, C. C. (submitted for review). An empirical typology of subjects within stage of change.

Winston, R. B. \& Miller, T. K. (1987). Student developmental task and lifestyle inventory manual. Athens, GA: Student Development Associates.

Zwick, W. R. \& Velicer, W. F. (1986). Comparison of five rules for determining the number of components to retain. Psychological Bulletin, 99 , 432-442. 
Table 3-1: Principal component loadings for exploratory analysis of URICA-A items

Component

\section{Scale 1: Precontemplation}

1 - As long as I do not drive getting drunk is all right.

2 - Having 4, 5, or more drinks at a time is OK for me.

3 - I think it is OK for me to drink a lot. .69

4 - If I am careful getting a little drunk causes no problems for me. .66

\section{Scale 2: Contemplation}

1 - It is clear to me that I must reduce my drinking.

2 - Although I have reduced my drinking already I must reduce it more. $\quad .70$

3 - I am building up my confidence to cut my drinking down soon . $\quad .67$

4 - My drinking bothers me more than it used to. $\quad .66$

5 - I am starting to realize that my drinking causes me difficulties.

6 - Some things in my life would be better if I drank less.

7 - I have a definite plan of action to reduce my drinking soon, $\quad .61$

8 - I am making a firm commitment to myself to stop drinking heavily very soon. $\quad .61$

9 - I am at the stage where I should think about drinking less alcohol. $\quad .60$

10 - I have started to wonder if my drinking is good for me.

11 - Although it is good that I am no longer drinking a lot it still feels strange .53 sometimes.

12 - Although I still drink a lot it is not as much fun as it used be.

\section{Scale 3: Maintenance}

1 - I do not feel a pull to drink like I used to.

2 - My old heavy drinking habits are fading in the past.

3 - I am not as tempted as I used to be to drink a lot.

4 - I am satisfied with my recent reduction in my drinking.

5 - 1 no longer think of myself as a heavy drinker. 
Table 3-2: Means, standard deviations, coefficient alphas, and scale intercorrelations for the URICA-A scales for both samples

Sample $1(\mathrm{~N}=294)$

\begin{tabular}{lcccccc}
\hline & & & & \multicolumn{3}{c}{ Correlations } \\
\cline { 6 - 7 } SCALE & Mean & SD & Alpha & PC & CONT & MAIN \\
\hline Precontemplation & 2.98 & .92 & .71 & -- & \\
Contemplation & 2.52 & .74 & .82 & $-.21^{*}$ & -- & \\
Maintenance & 3.40 & .87 & .79 & $-.27^{*}$ & .08 & - \\
\hline
\end{tabular}

* Correlation significant at $\mathrm{p}<.05$

Sample $2(\mathrm{~N}=322)$

\begin{tabular}{lcccccc}
\hline & & & & \multicolumn{3}{c}{ Correlations } \\
\cline { 6 - 7 } SCALE & Mean & SD & Alpha & PC & CONT & MAIN \\
\hline Precontemplation & 2.99 & .89 & .69 & -- & \\
Contemplation & 2.50 & .76 & .83 & $-.13^{*}$ & -- & \\
Maintenance & 3.37 & .85 & .77 & $-.11^{*}$ & $.12^{*}$ & - \\
\hline
\end{tabular}

* Correlation significant at $\mathrm{p}<.05$ 
Table 3-3: Cross-classification of Stage and Cluster

\begin{tabular}{|c|c|c|c|c|c|c|}
\hline \multirow[b]{2}{*}{ Cluster } & \multirow[b]{2}{*}{$\mathbf{N}$} & \multicolumn{5}{|c|}{ Percent of cluster in algorithmic stage } \\
\hline & & $\mathrm{PC}^{*}$ & Cont & Prep & Action & Maint \\
\hline PC-1 & 50 & $\underline{86}$ & 8 & 2 & 2 & 2 \\
\hline $\mathrm{PC}-2$ & 115 & $\underline{82}$ & 10 & 0 & 6 & 1 \\
\hline Immotive-PC & 51 & $\underline{73}$ & 8 & 0 & 8 & 12 \\
\hline Immotive-M & 41 & 24 & 10 & 0 & 27 & $\underline{39}$ \\
\hline Contemplation & 68 & $\underline{57}$ & 19 & 6 & 6 & 12 \\
\hline Preparation & 48 & $\underline{35}$ & 27 & 21 & 8 & 8 \\
\hline Action & 54 & 22 & 9 & 9 & 13 & $\underline{46}$ \\
\hline Maintenance & 27 & 7 & 7 & 4 & 15 & 67 \\
\hline
\end{tabular}

* Precontemplation group includes subjects from the Action for Acquisition stage ( $n=35$ )

Note: Percentages above $20 \%$ are in bold.

Highest percentage in each row is underlined. 
Table 3-4: Structural Coefficients for Discriminant Function Analysis

\begin{tabular}{cccc}
\hline URICA-A scales & Function 1 & & Function 2 \\
\cline { 3 - 4 } Precontemplation & -.603 & -.354 \\
Contemplation & .016 & .998 \\
Maintenance & .837 & .005 \\
\hline
\end{tabular}


Table 3-5: Discriminant Function Analysis: Cross-classification matrix by jackknifed procedure

Percentage classified into stage groups

\begin{tabular}{cccccc}
\cline { 2 - 5 } Algorithmic Stage & PC & CONT & PREP & ACTION & MAINT \\
\hline Precontemplation & 58 & 17 & 7 & 12 & 6 \\
Contemplation & 28 & 24 & 31 & 9 & 9 \\
Preparation & 5 & 28 & 52 & 5 & 10 \\
Action & & & & & \\
& 24 & 10 & 5 & 31 & \\
Maintenance & 5 & 2 & 8 & 25 & \\
\hline
\end{tabular}

Note: Highest percentage in each row is in bold. 
Table 3-6: Distributions of categorical variables across Adjusted Clusters

\begin{tabular}{|c|c|c|c|c|c|c|}
\hline \multirow{2}{*}{$\begin{array}{l}\text { ADJUSTED } \\
\text { CLUSTER }\end{array}$} & \multicolumn{2}{|c|}{ Total } & \multicolumn{2}{|c|}{ Women } & \multicolumn{2}{|c|}{ Men } \\
\hline & $\mathrm{N}$ & $\%$ & $\mathrm{~N}$ & $\%$ & $\mathrm{~N}$ & $\%$ \\
\hline Precontemplation & 195 & 57.9 & 111 & 52.1 & 84 & 67.7 \\
\hline Contemplation & 56 & 16.6 & 41 & 19.2 & 15 & 12.1 \\
\hline Preparation & 27 & 8.0 & 13 & 6.1 & 14 & 11.3 \\
\hline Action & 37 & 11.0 & 30 & 14.1 & 7 & 5.6 \\
\hline Maintenance & 22 & 6.5 & 18 & 8.4 & 4 & 3.2 \\
\hline
\end{tabular}

Note: Male/Female differences are significant: $\chi^{2}(4)=16.72, p<.01$

\begin{tabular}{|c|c|c|c|c|c|c|c|c|}
\hline \multirow{3}{*}{$\begin{array}{c}\text { ADJUSTED } \\
\text { CLUSTER }\end{array}$} & \multicolumn{8}{|c|}{ Living Situation } \\
\hline & \multicolumn{2}{|c|}{ Dormitory } & \multicolumn{2}{|c|}{ Greek } & \multicolumn{2}{|c|}{ Off Campus } & \multicolumn{2}{|c|}{ With Parents } \\
\hline & $\mathrm{N}$ & $\%$ & $\mathrm{~N}$ & $\%$ & $\mathrm{~N}$ & $\%$ & $\mathrm{~N}$ & $\%$ \\
\hline Precontemplation & 84 & 60.9 & 28 & 60.9 & 64 & 57.7 & 18 & 47.4 \\
\hline Contemplation & 25 & 18.1 & 10 & 21.7 & 15 & 13.5 & 6 & 15.8 \\
\hline Preparation & 6 & 4.4 & 4 & 8.7 & 12 & 10.8 & 4 & 10.5 \\
\hline Action & 16 & 11.6 & 4 & 8.7 & 8 & 7.2 & 7 & 18.4 \\
\hline Maintenance & 7 & 5.1 & 0 & 0.0 & 12 & 10.8 & 3 & 7.9 \\
\hline
\end{tabular}

Note: Differences across living situations are non-significant

\begin{tabular}{|c|c|c|c|c|c|c|c|c|}
\hline \multirow{3}{*}{$\begin{array}{c}\text { ADJUSTED } \\
\text { CLUSTER }\end{array}$} & \multicolumn{8}{|c|}{ Class } \\
\hline & \multicolumn{2}{|c|}{ Freshman } & \multicolumn{2}{|c|}{ Sophomore } & \multicolumn{2}{|c|}{ Junior } & \multicolumn{2}{|c|}{ Senior } \\
\hline & $\mathrm{N}$ & $\%$ & $\mathrm{~N}$ & $\%$ & $\mathrm{~N}$ & $\%$ & $\mathrm{~N}$ & $\%$ \\
\hline Precontemplation & 39 & 65.0 & 72 & 62.1 & 33 & 55.0 & 41 & 47.7 \\
\hline Contemplation & 12 & 20.0 & 19 & 16.4 & 10 & 16.7 & 15 & 17.4 \\
\hline Preparation & 3 & 5.0 & 6 & 5.2 & 6 & 10.0 & 10 & 11.6 \\
\hline Action & 4 & 6.6 & 12 & 11.2 & 7 & 11.7 & 11 & 12.8 \\
\hline Maintenance & 2 & 3.3 & 6 & 5.2 & 4 & 6.7 & 9 & 10.5 \\
\hline
\end{tabular}

Note: Differences across classes are non-significant 
Table 3-7: Means and standard deviations for continuous variables across the Adjusted Clusters

\begin{tabular}{|c|c|c|c|c|c|c|c|c|}
\hline \multirow{3}{*}{$\begin{array}{c}\text { ADJUSTED } \\
\text { CLUSTER }\end{array}$} & \multirow{2}{*}{\multicolumn{2}{|c|}{ AGE }} & \multicolumn{6}{|c|}{ Consumption Variables } \\
\hline & & & \multicolumn{2}{|c|}{ DAYS } & \multicolumn{2}{|c|}{ DRINKS } & \multicolumn{2}{|c|}{ INTOX } \\
\hline & Mean & $S D$ & Mean & $\mathrm{SD}$ & Mean & $\mathrm{SD}$ & Mean & $\mathrm{SD}$ \\
\hline Precontemplation & 20.19 & 1.36 & 11.77 & 5.68 & 6.88 & 2.90 & 6.21 & 2.34 \\
\hline Contemplation & 20.21 & 1.37 & 11.34 & 6.35 & 6.48 & 3.71 & 5.91 & 2.04 \\
\hline Preparation & 21.00 & 1.48 & 8.82 & 5.53 & 6.52 & 2.36 & 6.04 & 2.07 \\
\hline Action & 21.34 & 2.15 & 4.00 & 2.90 & 3.86 & 1.82 & 4.44 & 2.05 \\
\hline Maintenance & 21.25 & 2.14 & 3.91 & 4.51 & 1.86 & 1.24 & 3.95 & 1.36 \\
\hline
\end{tabular}

\begin{tabular}{|c|c|c|c|c|c|c|}
\hline \multirow{3}{*}{$\begin{array}{c}\text { ADJUSTED } \\
\text { CLUSTER }\end{array}$} & \multicolumn{6}{|c|}{ Alcohol Related Experiences } \\
\hline & \multicolumn{2}{|c|}{ EXCESS } & \multicolumn{2}{|c|}{ DISTRESS } & \multicolumn{2}{|c|}{ PROBLEM } \\
\hline & Mean & $\mathrm{SD}$ & Mean & $\mathrm{SD}$ & Mean & SD \\
\hline Precontemplation & 2.44 & 1.02 & 1.24 & 1.00 & .43 & .61 \\
\hline Contemplation & 2.52 & 1.09 & 1.93 & 1.38 & .62 & .81 \\
\hline Preparation & 2.51 & 1.08 & 2.13 & 1.12 & .62 & .80 \\
\hline Action & 1.42 & .96 & 1.15 & .96 & .22 & .62 \\
\hline Maintenance & 0.72 & .75 & .54 & .68 & .06 & .13 \\
\hline
\end{tabular}

\begin{tabular}{|c|c|c|c|c|c|c|}
\hline \multirow{3}{*}{$\begin{array}{c}\text { ADJUSTED } \\
\text { CLUSTER }\end{array}$} & \multicolumn{6}{|c|}{ Psychosocial Maturity } \\
\hline & \multicolumn{2}{|c|}{$\begin{array}{c}\text { PEER } \\
\text { RELATIONS }\end{array}$} & \multicolumn{2}{|c|}{$\begin{array}{l}\text { EMOTIONAL } \\
\text { AUTONOMY }\end{array}$} & \multicolumn{2}{|c|}{$\begin{array}{l}\text { LIFESTYLE } \\
\text { PLANNING }\end{array}$} \\
\hline & Mean & $\mathrm{SD}$ & Mean & $\mathrm{SD}$ & Mean & SD \\
\hline Precontemplation & 2.67 & .56 & 2.84 & .60 & 3.12 & .60 \\
\hline Contemplation & 2.69 & .66 & 2.88 & .66 & 3.00 & .71 \\
\hline Preparation & 2.82 & .52 & 2.90 & .50 & 3.00 & .56 \\
\hline Action & 2.68 & .43 & 2.86 & .60 & 3.59 & .80 \\
\hline Maintenance & 2.63 & .52 & 2.84 & .52 & 3.23 & .63 \\
\hline
\end{tabular}


Figure 3-1:_Structural model for URICA-A scales

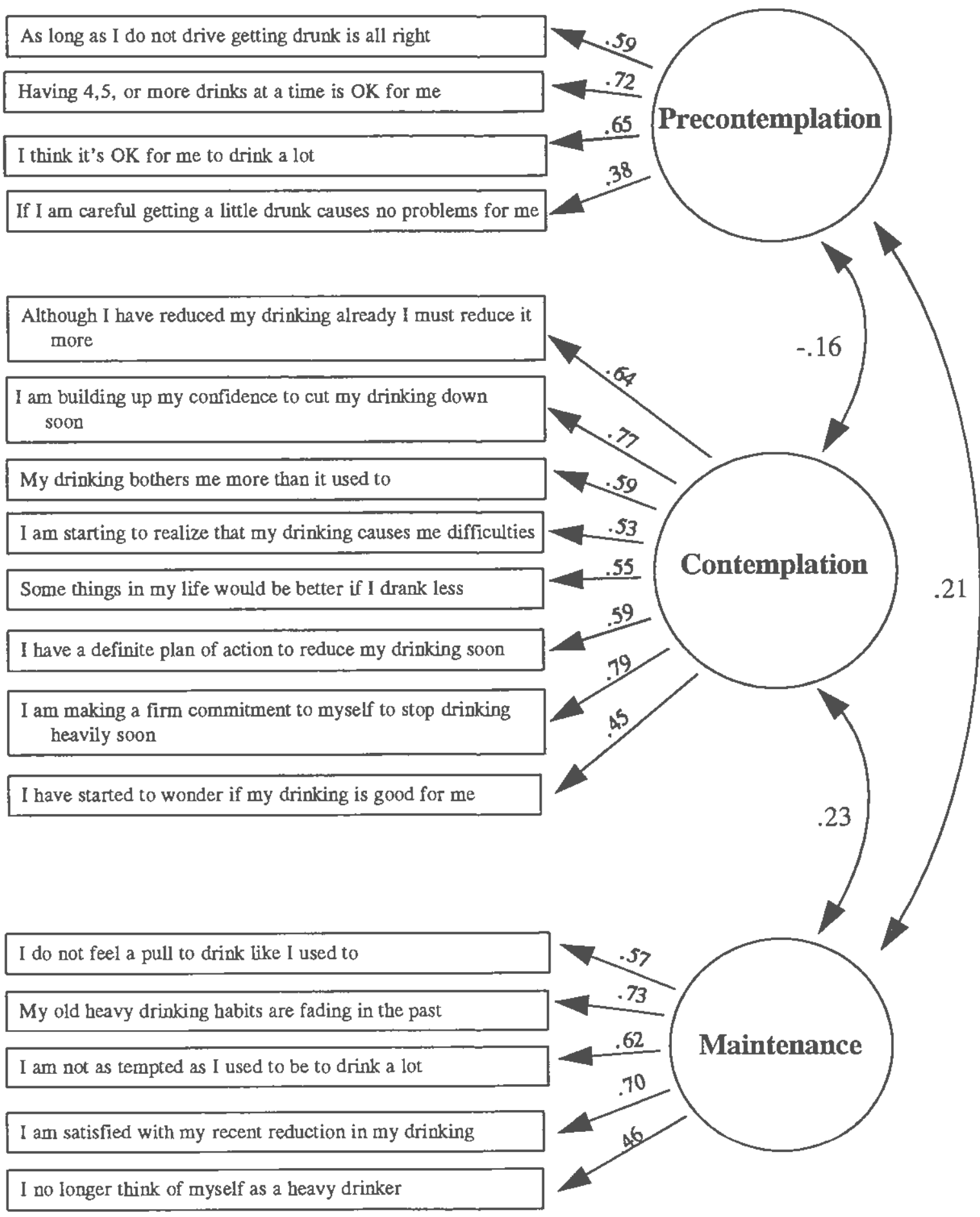


Figure 3-2: Profiles of PC-1 and PC-2 URICA-A clusters

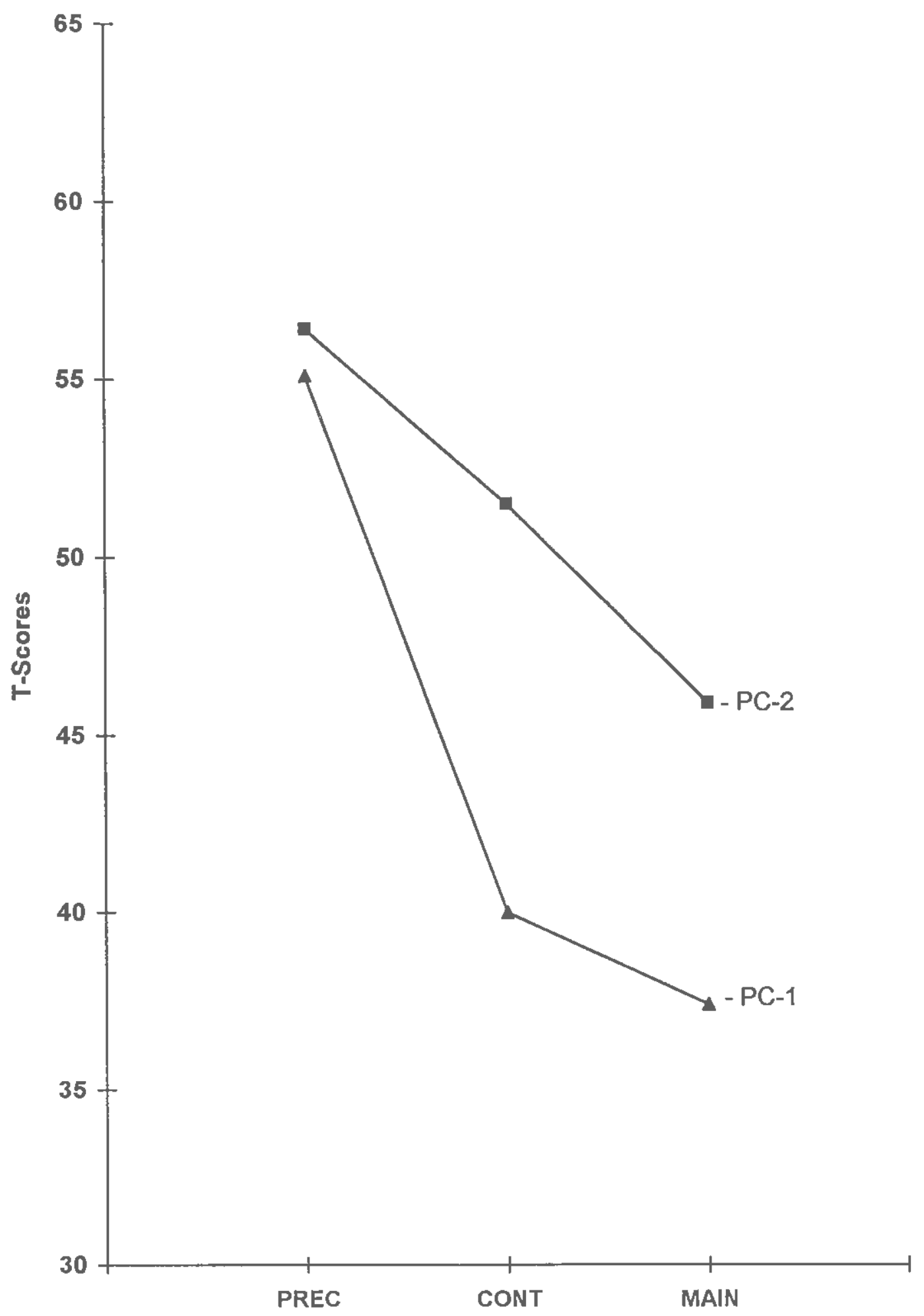


Figure 3-3: Profiles of Immotive-PC and Immotive-M URICA-A clusters

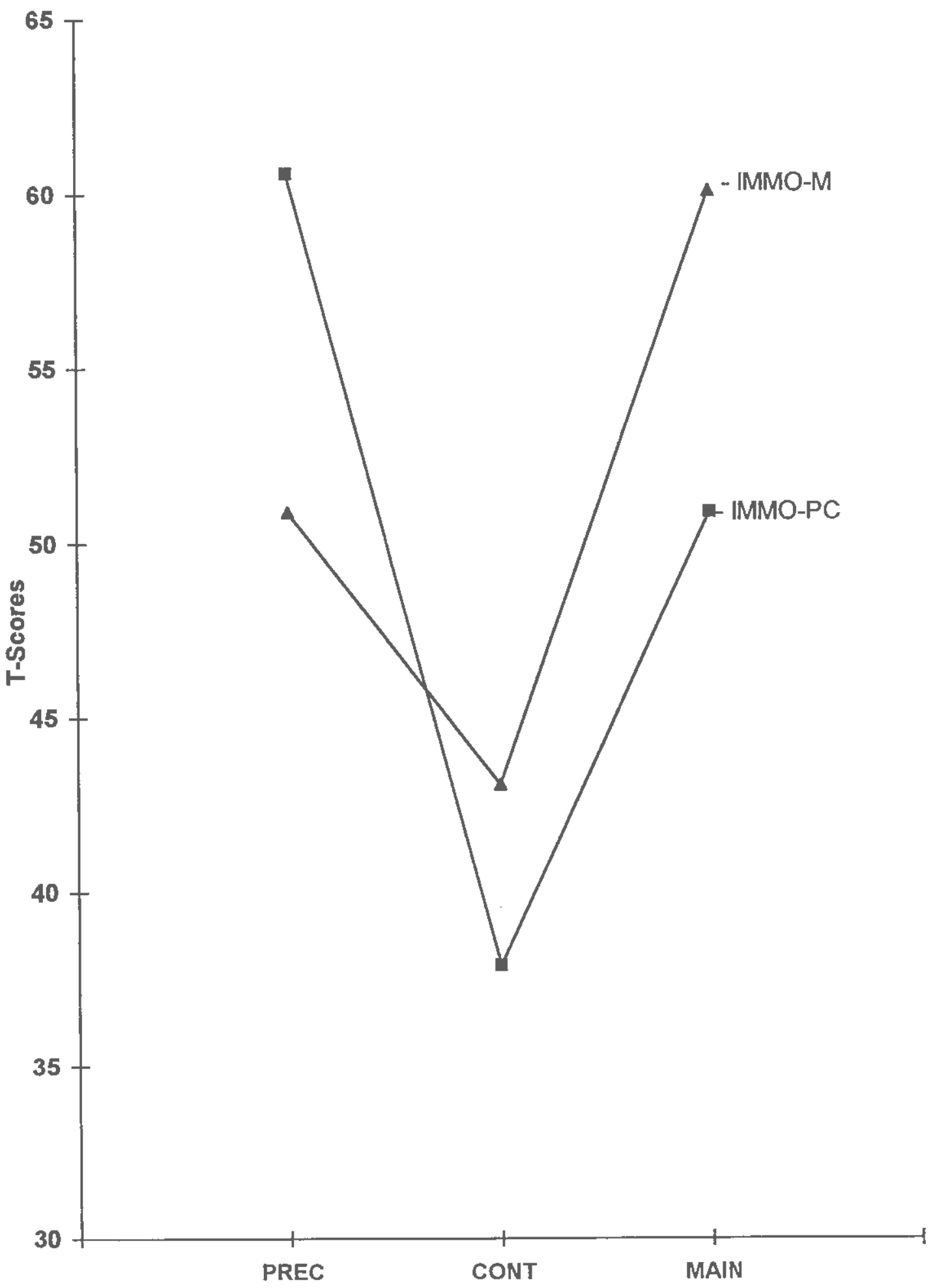


Figure 3-4: Profiles of Contemplation and Preparation URICA-A clusters

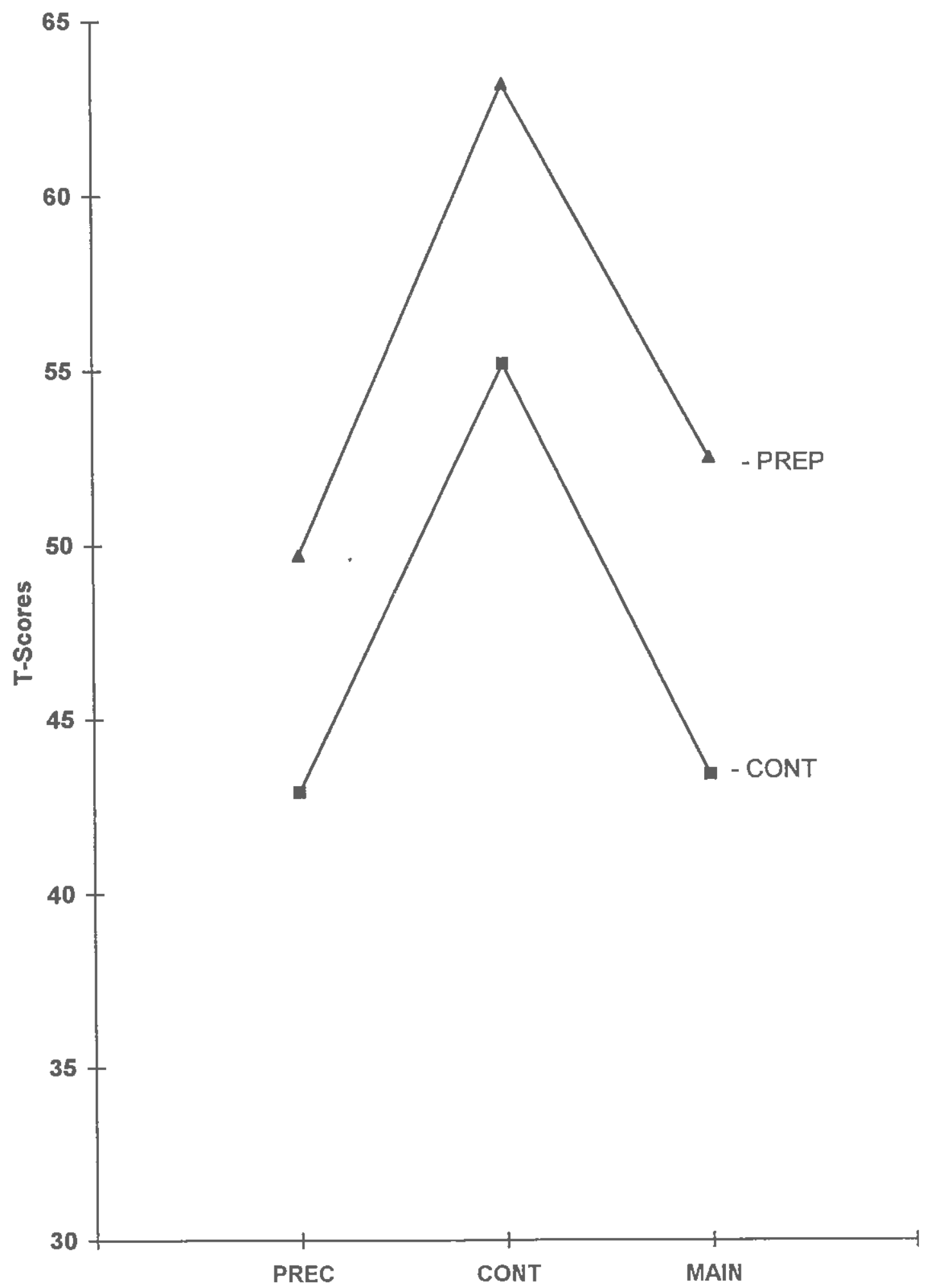


Figure 3-5: Profiles of Action and Maintenance URICA-A clusters

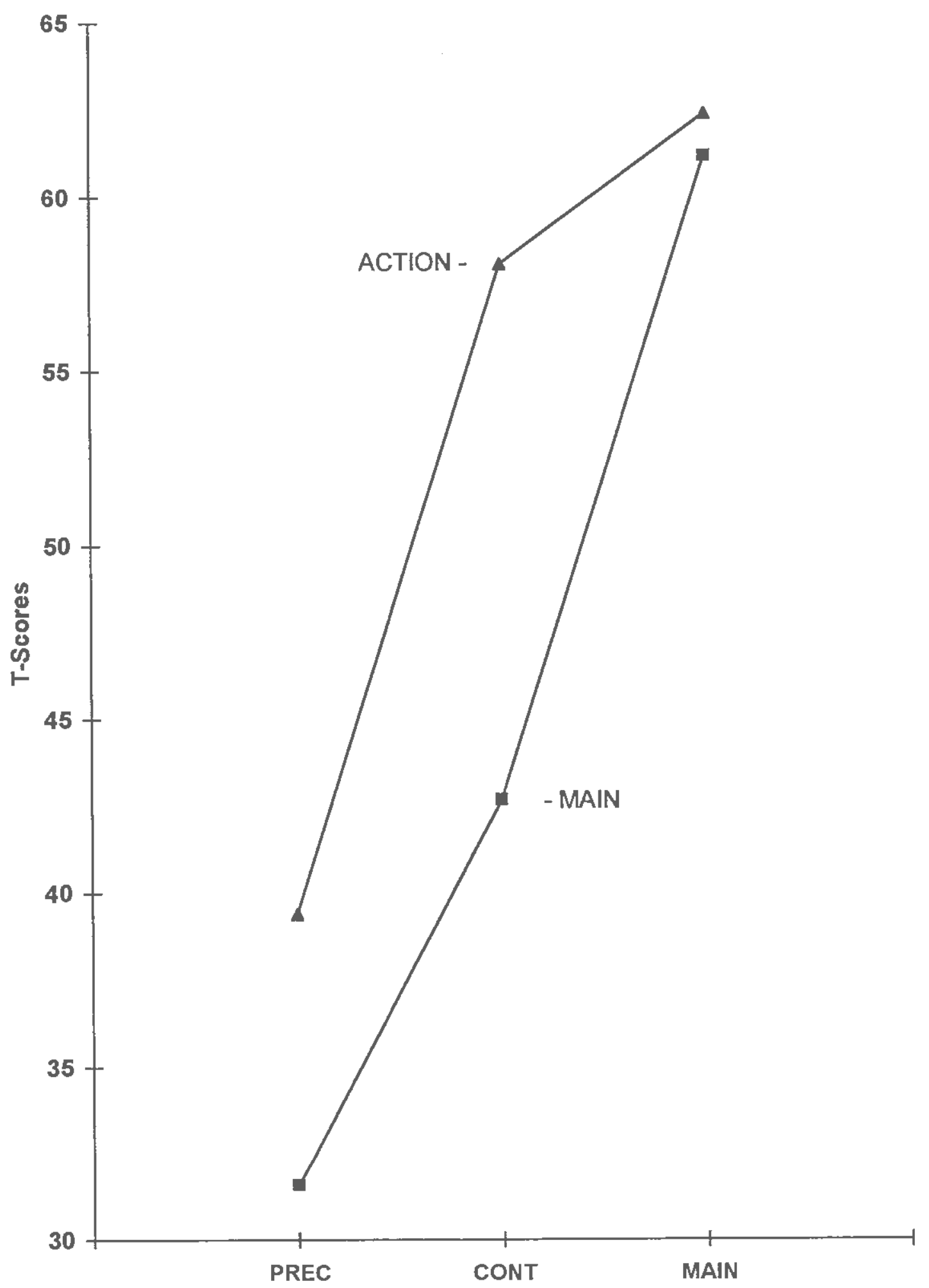




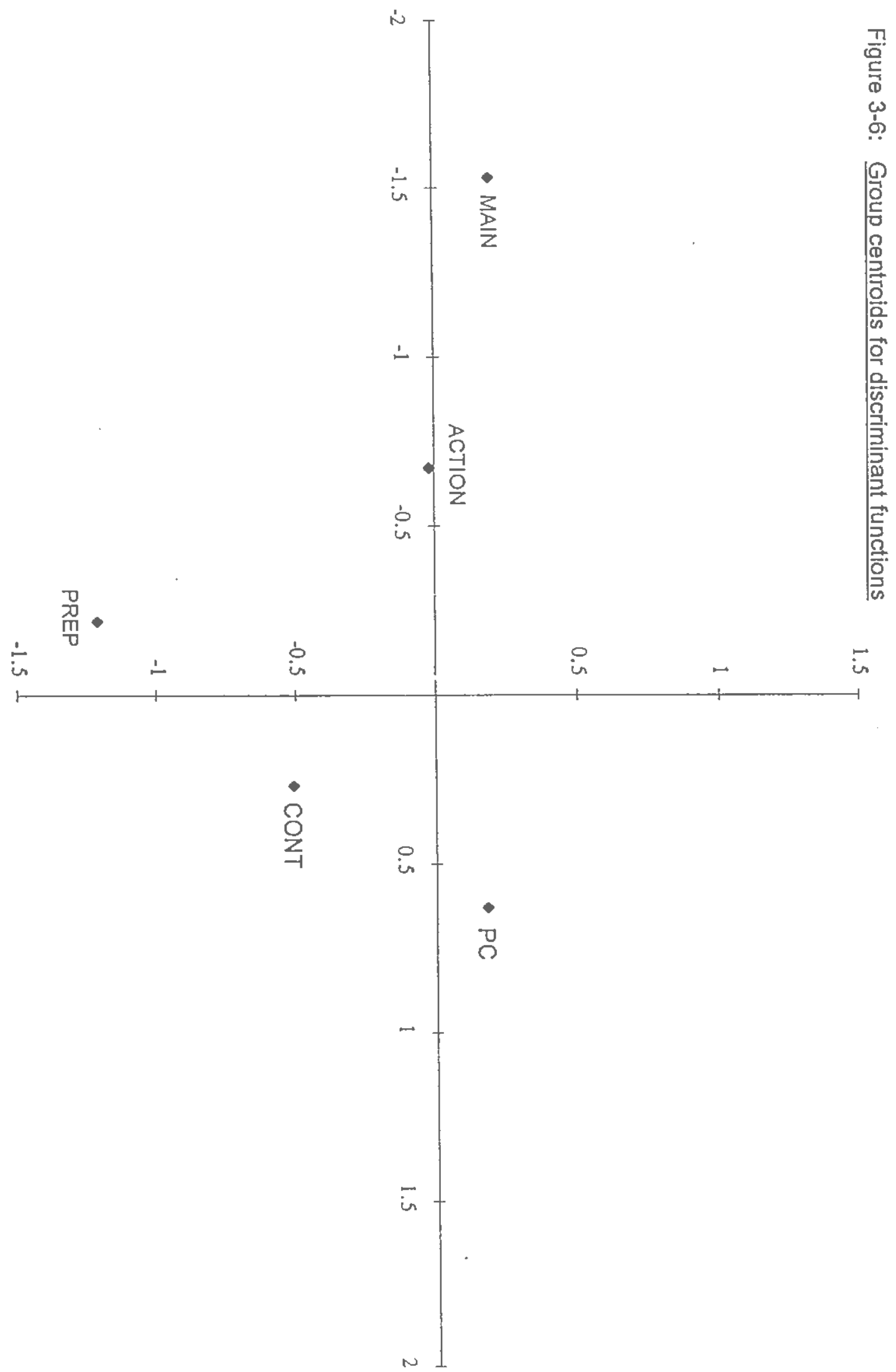


Figure 3-7 : Age by Adjusted Cluster

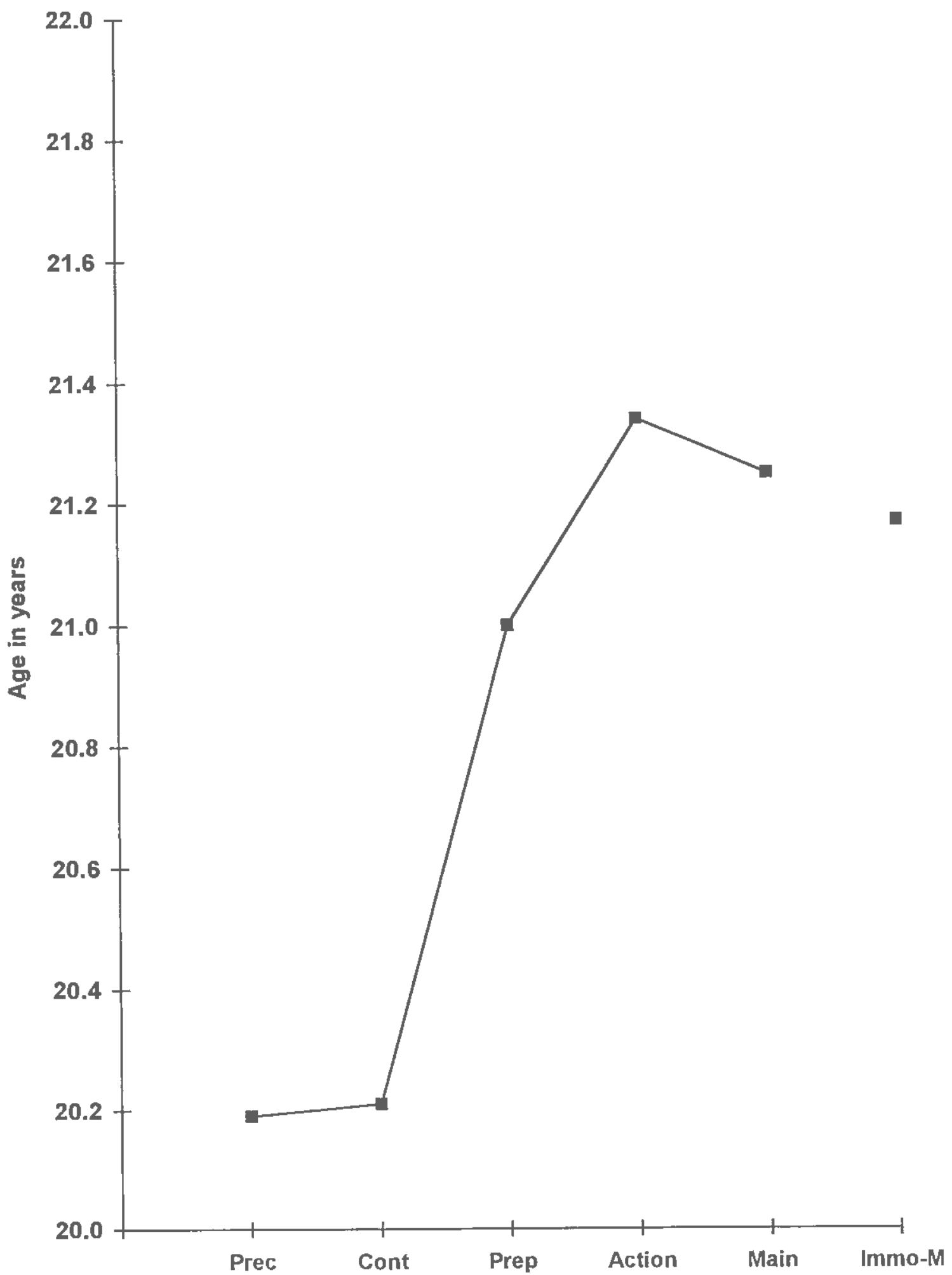


Figure 3-8 : Consumption variables by Adjusted Clusters

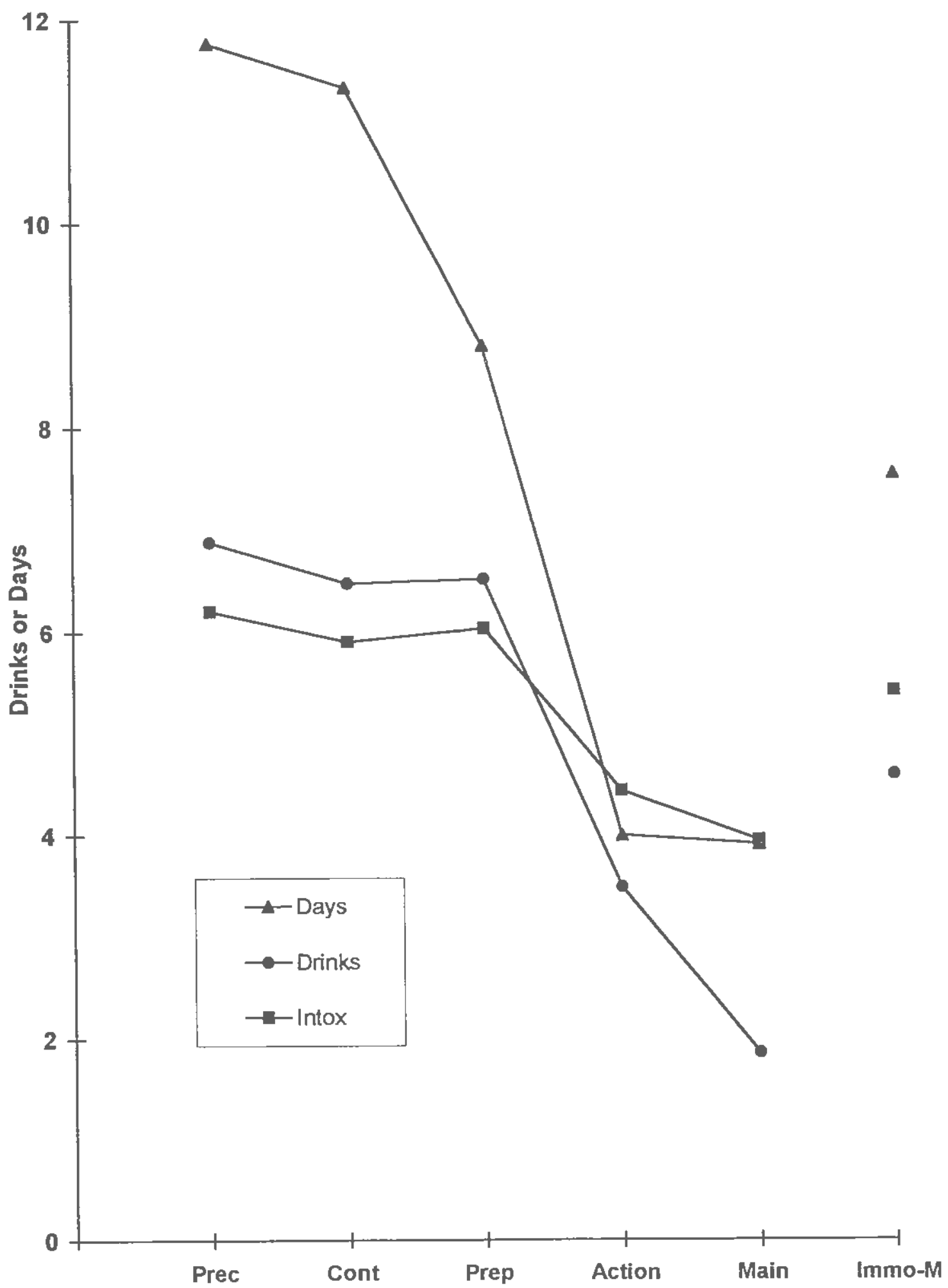


Figure 3-9: Alcohol-Related Experiences scales by Adjusted Cluster

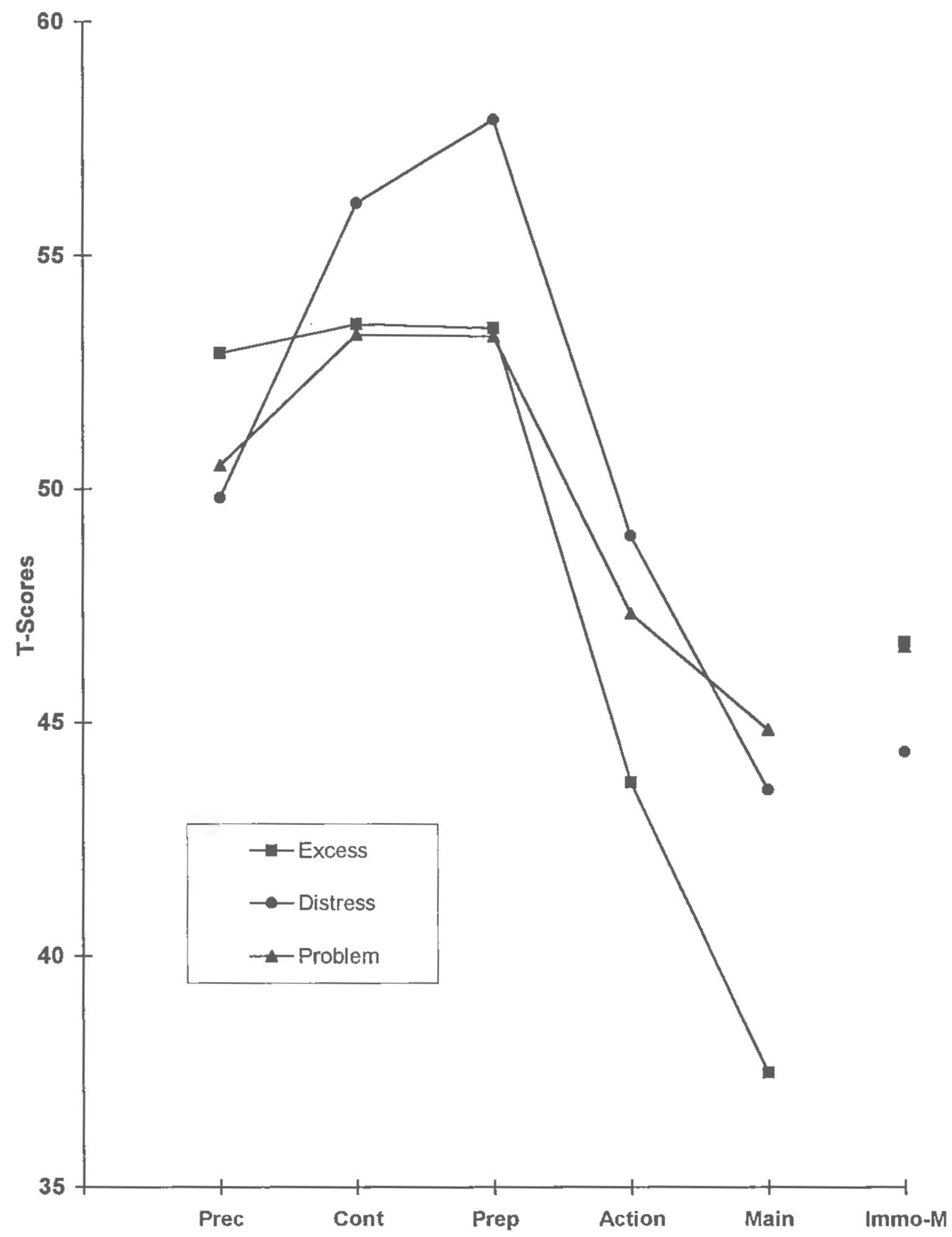


Figure 3-10 : Psychosocial development scales by Adjusted Cluster

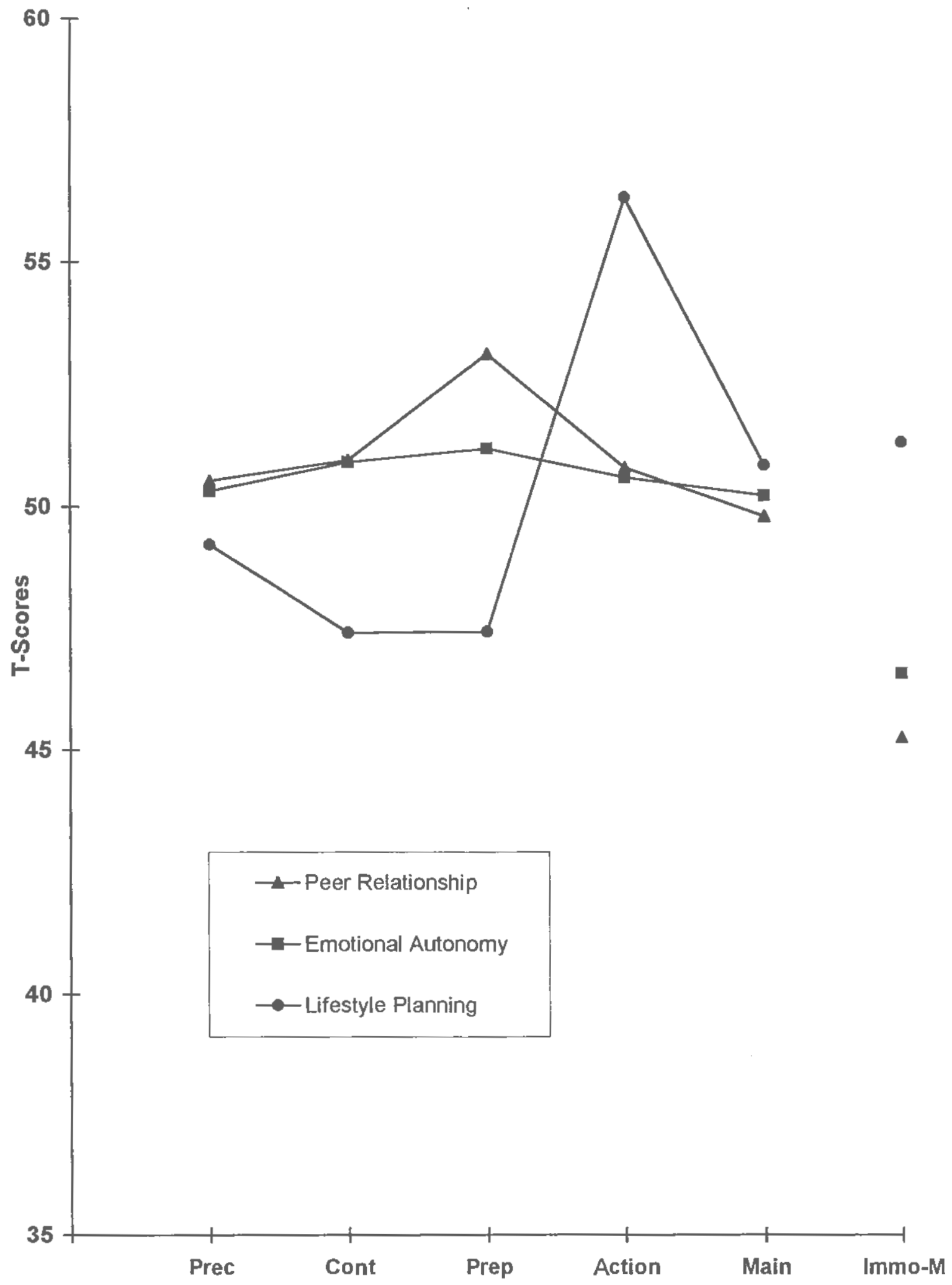




\section{PART IV. STUDY 3: DECISIONAL,BALANCE FOR ALCOHOL USE}

\section{INTRODUCTION}

The prevalence of alcohol use and its associated problems on college campuses in the United States are well documented. Most students drink (Kraft, 1988) and many experience negative consequences to their drinking. In a recent study of one northeastern college it was found that in the previous week $25 \%$ of students reported having a hangover, $7.5 \%$ had vomited, and $4 \%$ had a blackout (Meilman, Stone, Gaylor, \& Turco, 1990). Although there are many factors that influence the likelihood that a student will suffer a negative consequence of her drinking including her tolerance to alcohol, her tendency to take risks, her expectancies of the effects of alcohol, the nature of her peer group, and perhaps, also her luck, at a more primary level the risk of negative consequences is largely determined by the decision to drink at all, and if so how much to drink. Yet, the consideration of this decision making process has received relatively little research attention.

One model of behavior change that contains a decision making construct is the Transtheoretical Model of Change (TTM). This model has gained wide acceptance as a useful model for the investigation of behavior change and has been applied to many behaviors. One of the five constructs of this model is Decisional Balance, which was developed from Janis and Mann's $(1968,1977)$ conflict theory of decision making and was initially investigated empirically within the Transtheoretical Model with smokers (Velicer, DiClemente, Prochaska, \& Brandenburg, 1985).

Janis and Mann's theory posited that an individual's decision to behave resulted from an assessment of the losses and gains associated with the behavior in question. They conceptualized four categories of losses and gains that individuals evaluate: 1) utilitarian losses or gains for oneself; 2) utilitarian losses and gains for others; 3) self-approval or selfdisapproval; 4) approval or disapproval from significant others. Research on Decisional 
Balance within the context of the TTM has typically attempted to measure all eight of these categories (gains and losses for each category), but has consistently found just two orthogonal factors: the Pros and Cons. One exception has been Redding's work on safe-sex practices (Redding, 1993). This research found four categories with both the Cons and Pros composed of two sub-scales representing consequences to self and to others. It is not surprising that this result was found for a behavior that so clearly involves another.

Another construct of the TTM is the Stages of Change. This construct is fundamental to the model as it is most closely related to behavior change and provides a heuristic structure to investigate the other constructs of the model, including Decisional Balance. There are five primary stages: Precontemplation (not considering changing the target behavior),

Contemplation (intending to change in the next six months), Preparation (planning change in the next 30 days), Action (having changed in the last six months), and Maintenance (having changed the behavior more than six months ago). The stages can be conceptualized for both the acquisition or cessation of behaviors. ${ }^{1}$ These stages have been validated in many different behavioral domains, and where longitudinal research has been done, have been highly predictive of future behavior change (Prochaska, DiClemente, Velicer, Ginpil, \& Norcross, 1985).

It has been found that Decisional Balance has a very consistent qualitative and quantitative relationship to the Stages of Change. Across 12 problem behaviors it has been found that the standardized values of these two scales tend to cross-over before action is taken (Prochaska, Velicer, Rossi, Goldstein, Marcus, Rakowski et al., 1994). Also two quantitative relationships have been identified, named the strong and weak principles (Prochaska, 1994). The strong principle states that movement from Precontemplation to Action is accompanied by a one standard deviation increase in the Pros of changing the behavior (or Cons of the behavior itself). The weak principle states that a corresponding stage movement is

\footnotetext{
${ }^{1}$ - In this study unless otherwise specified 'Stage' refers to the Stages of Cessation
} 
accompanied by a one-half standard deviation decrease in the Cons of changing the behavior (or Pros of the behavior). Such a quantitative relationship is rarely found in psychology.

This construct has proven to be most predictive of early stage movement (DiClemente, Prochaska, Velicer, Velasquez, \& Rossi, 1991). Having accurate measures of Decisional Balance is likely to be useful in designing new interventions that target early stage subjects. This is especially important for immoderate alcohol use in college, as the majority of college students are in the stages of Precontemplation or Contemplation (Migneault, Stevenson \& Velicer, 1994; or see Study I of this dissertation).

There has been limited research on this construct for behaviors involving alcohol use. In one survey, 220 vocational students completed a set of 37 items representing the Pros and Cons of excessive aicohol use. Principal components analysis found, as in other research, only two factors, the Pros and Cons of alcohol use. The relationship of these variables to the Stages of Change, as predicted, conformed to previous research (Migneault, Pallonen, \& Velicer, 1994). Another study developed a Decisional Balance instrument on a population of inpatient alcohol patients in a VA hospital (King \& DiClemente, personal communication, March, 1993). Again, two orthogonal factors were found, the Pros and Cons of Drinking. These scales were meaningfully related to both TTM constructs and other drinking related variables.

There is another body of research that is not based on the TTM but is related to the Decisional Balance construct. Brown and colleagues have worked on measuring the alcohol expectancies that many laboratory studies have shown to mediate the effects of alcohol (Marlatt \& Rohsenow, 1980). They developed very large item sets of possible positive effects of drinking on anyone and used a yes/no answer format (Brown, Goldman, Inn, \& Anderson, 1980). Although this somewhat less than optimal instrument development method led to long scales, some of which had low face validity, and no assessment of negative expectancies, the scales have provided many positive research results. The instrument has six scales labeled general positive effect, enhances social and physical experiences, enhances sexual 
performance, increases power and aggression, increases social assertiveness, and reduces tension. They have found that positive expectancies predict level of drinking, and that patterns across the scales predict type of drinking (Brown, 1985). For example, those who strongly believe that drinking enhances social pleasure were more likely to be social drinkers, whereas those who believed most strongly that it reduced tension were more likely to be problem drinkers.

Although this research seems to be assessing a general attitude rather than a personal assessment of the consequences of one's own drinking, the fact that these scales have been powerful predictors of other important variables, lends support to the utility of this type of investigation. It is of additional interest that separate factors were found in a domain that seems closely related to the Pros of alcohol use, whereas this variable has been consistently uni-dimensional across many other behavioral domains.

The purpose of the present study is to develop a psychometrically sound and valid Decisional Balance instrument for drinking that is applicable to college populations. Such an instrument will be useful in the investigation of college alcohol drinking patterns and the naturally occurring change in these patterns and to help design programs to move students to less risky levels of alcohol consumption. 


\section{METHOD}

\section{SUBJECTS}

Subjects were 629 students surveyed at the University of Rhode Island in the fall of 1993 and spring of 1994 between the ages of 18 and 25 years and 11 months. Most of the subjects were recruited from undergraduate classes in the psychology department, although a variety of other classes were also sampled including physical education, pharmacology, mutrition, and sociology. A small percentage was recruited from the fraternities and the university health center.

The sample was $66.3 \%$ female, and $94 \%$ white. The distribution across the classes was $17.4 \%$ freshmen, $32.2 \%$ sophomores, $19.1 \%$ juniors, $26.2 \%$ seniors, and $5.3 \%$ either fifth-year or non-matriculated. The average age was 21.3 years. Of these students $47 \%$ were classified as in-state and $39 \%$ lived in dormitories, $10 \%$ lived in fraternities or sororities, and $51 \%$ lived off campus.

The use of alcohol in this sample was substantial. Using a criterion of at least one drinking occasion in the last 30 days, $92 \%$ were classified as active drinkers. These subjects drank on average 8.6 days a month and consumed on average 5.3 drinks per occasion. There were substantial differences in drinking by gender with drinking women averaging 7.3 days per month and 4.4 drinks per occasion. For men these figures were 11.0 days and 6.9 drinks. Drinking women reported an average maximum amount drunk on any occasion in the past month as 6.8 , and men as 12.1 .

\section{INSTRUMENTS}

The survey administered contained 282 questions, including, of specific interest to this study, the following item sets: The full survey instrument is presented in Appendix A. 


\section{Demographics}

A set of 22 items asked about basic demographic information and drinking history. Variables examined in this study included age, gender, number of days in the last month that alcohol was consumed (Days), the number of drinks consumed during a typical drinking occasion (Drinks), and the number of drinks consumed before subjects start to feel intoxicated (Intoxication).

\section{Decisional Balance Item Set}

A set of 25 items hypothesized to be measures of either the Pros or the Cons of drinking was the focus of this investigation. A large initial pool of items was generated from a number of sources including adapting items from the Alcohol Expectancies Questionnaire (Brown et al., 1980), from an instrument developed by King and DiClemente (personal communication, March, 1993), and a Decisional Balance instrument developed on a sample of Vocational High School students (Migneault et al., 1994). Additional items were developed by this author to fully assess the possible range of considerations of college students. Of these items 66 were pilot tested on a sample of 223 college students in a preliminary study. A principal components analysis was done to assess structure and component loadings. On the basis of these data, 25 items were chosen for a final item set. Subjects were asked to rank the importance of each item in their decisions about how much they drank or whether to drink at all using a 5-point Likert scale with $I=$ Not at all important and $5=$ Extremely important.

\section{Stages of Change}

Algorithmic assessment. The staging algorithm used answers from a branched set of five items to classify subjects into one of nine groups that represented the five Stages of Acquisition and the five Stages of Cessation with one group representing both the Maintenance Stage of Acquisition and the Precontemplation Stage of Cessation. In the present study only the Stages of Cessation were investigated $(\mathrm{N}=426)$. The algorithm used was 
developed and investigated in Study I and used a criterion of usually drinking 4 or more drinks at least once in a typical week for women and 5 or more for men. The development and investigation of this staging method is presented in Study I.

Continuous measure. Three scales which compose the 21-item University of Rhode Island Change Assessment for Alcohol instrument (URICA-A) were used to assign students to one of five Stages of Cessation. These scales included a Precontemplation scale which measures acceptance of heavy drinking, a Contemplation scale which measures ambivalence about one's drinking habits and plans to change them, and a Maintenance scale which measures attitudes consistent with having reduced one's drinking and maintained them at more moderate levels. Scale alphas are .70 for the Precontemplation scale, .83 for the Contemplation scale, and .78 for the Maintenance scale.

Scale scores were entered into a cluster analysis and an eight cluster solution was chosen as being most useful. A combination of cluster membership and algorithmic stage was used to assign subjects to a Stage of Cessation $(\mathrm{N}=337)$. These groups were labeled Adjusted Clusters. Details of their development and investigation are presented in Study II.

\section{Alcohol-Related Experiences}

Three scales, developed in Study I, that measure types of negative alcohol related experiences were included as a central validity variable. The scales are composed of 23 items developed from an original set of 31 items and are labeled Excess, Distress, and Problem. The three scales have moderate inter-scale correlations ranging from .37 and .44 and high internal consistencies as measured by Cronbach's Alpha ranging from .80 to .88. The Excess scale is hypothesized to measure problems associated with short-term excessive or binge drinking. The Distress scale measures experiences of emotional or introspective distress, and the Problem scale measures consequences of alcohol consumption that are associated with long term problem drinking. 
Subjects were asked to circle the number of times in the last 12 months that they had had each experience related to their alcohol consumption. The following 6-point response scale was provided: $0,1,2,3-5,6-9,10$ or more times.

\section{PROCEDURE}

The survey for this study was included within a larger survey that assessed additional aspects of drinking behaviors and attitudes. Informed consent was obtained and subjects were assured of the confidentiality of their responses (See Appendix B for a copy of the consent form). Surveys were completed during class time, or subjects took the survey home and returned it. Most, although not all, of those doing the survey on their own time received a small amount of class credit for completing the survey. A few students who completed the survey at the health services center were given a pen emblazoned with the university name in exchange for completing the survey. 


\section{RESULTS}

\section{EXPLORATORY COMPONENT ANALYSIS}

Subjects who had missing data on more than 6 of the 25 Decisional Balance items were eliminated from the analysis leaving $96.5 \%$ of the sample $(\underline{N}=607)$. The sample was then randomly split into two subsamples. An exploratory principal components analysis (PCA) was conducted on the $25 \times 25$ matrix of item intercorrelations generated from sample 1 using pair-wise deletion $(\underline{N}=302)$. The number of components to retain was determined by comparing the results of three procedures that have been shown to be valid predictors of the correct dimensionality of an item set (Zwick \& Velicer, 1986). The Scree procedure (Catell, 1966) and the Minimum Average Partial (Velicer, 1976) suggested three components whereas Parallel Analysis (Horn 1968, Allen \& Hubbard, 1986) indicated that two was the correct number of components to extract. Both the two and three component solutions were investigated.

The two component solution clearly represented the Pros and Cons of alcohol use. Both orthogonal (varimax) and oblique (direct quartimin) rotations were performed. These solutions produced virtually identical results and the varimax solution was chosen for further analysis. One item was eliminated because it loaded on both components. Four other items were eliminated because of relatively low loadings and to shorten the final scale to reduce subject response burden. This resulted in a 20 -item scale, with 10 items in each subscale. The two components explained $53.4 \%$ of the variance of the reduced item set. Cronbach's Coefficient Alpha for sample 1 was .93 for the Pros subscale and .86 for the Cons subscale. Items with component loadings are presented in Table 4-1.

In the three component solution the Cons separated into two components. These two components were moderately correlated in an oblique rotation $(r=.26)$. The larger of the two Cons components was composed of five items measuring the importance of the risk of incurring harm due to alcohol consumption, such as hurting someone else, having legal 
troubles, or becoming addicted. The one item that does not ask explicitly about future risk is "Drinking is bad for my health", which implies the risk of illness or disability. The other Cons component was composed of three high loading items which measure the importance of actual negative emotional reactions to alcohol consumption such as feeling out of control, feeling down, and having lower self regard as a result of drinking. The remaining four items were complex across the two Cons scales.

It is unclear from this item set and the loading pattern whether subjects distinguished these components more on the basis of risk of negative effects versus actual negative effects or on the dimension of emotional distress versus other types of more practical harm. Future research that includes items that have other combinations of these attributes could help solve this question. Items such as "Drinking costs money" (actual and practical) or "drinking might give me emotional problems" (potential and emotional) could be investigated. The two Cons scales were label Cons-P for potential/practical and Cons-A for actual/affectual to capture both distinguishing dimensions.

The Pros scale was reduced to the same 10 items used in the two component solution and the four complex Con items were deleted resulting in an 18-item instrument. These three components explained $61.3 \%$ of the variance of this item set. Items with component loadings for the three component solution are presented in Table 4-2. For Sample 1 Cronbach's Coefficient Alpha was .84 for the Cons-P subscale and .62 for the Cons-A subscale.

\section{CONFIRMATORY FACTOR ANALYSIS}

To further evaluate both solutions confirmatory factor analysis using EQS structural modeling program (Bentler, 1989) was performed using the hold out sample $(\underline{N}=305)$. Six models were initially run. For both solutions, three models were run: a null model, a correlated factor model, and a model without correlations between the Pros and the Cons (the three factor model included a correlation between the two Cons factors). The null model posits the items function as independent variables and is not expected to fit the data but 
generates a set of statistics with which the other models can be compared. A set of fit indices including Comparative Fit Index (CFI), Non-Normed Fit Index (NNFI), Root Mean Square (RMS), and Incremental Fit Index, type 2 (IFI2) were calculated to assess model fit. Since the CFI, NNFI and IFI2 produced a similar pattern of results, only the IFI2 and RMS are reported here.

The two factor correlated and uncorrelated models had identical values for the IFI2 to two decimal places $(\mathrm{IFI} 2=.89)$, but the RMS for the correlated model was superior $(\mathrm{RMS}=.047$ versus .068 ). Furthermore the difference between the Chi-square statistics for these models was significant $\left(\chi^{2}(1)=5.65, p<.05\right)$, suggesting that the model improvement gain with the correlated model was significant. The correlation between the two factors was small $(r=.19)$ suggesting that the Pros and Cons are Iargely, but not fully, independent. The model is presented in Figure 4-1.

The three factor solution produced similar results. The correlated model fit the data better than the partially correlated model (Correlated model: IFI2 $=.92$, RMS $=.046$; Partially correlated modeI: IFI2 $=.91, \mathrm{RMS}=.072$ ). The chi-squared difference was significant $\left(\chi^{2}(1)=11.25, \mathrm{~g}<.001\right)$ These results suggests that the correlated model is superior in terms of model fit. This model is presented in Figure 4-2.

Additionally a hierarchical model was fit to the data. In this model both the Cons-P and the Cons $\sim \mathrm{A}$ were loaded on a higher order Cons factor which was allowed to correlate with the Pros factor. This model had identical model fit and item loading pattern as the fully correlated three factor model suggesting that it is essentially another way of conceptualizing the relationship between the factors. This model is presented in Figure 4-3.

Although both correlated models had good model fit, the three factor solution was chosen as the basis for investigating external validity evidence. The Pros scale is identical in both solutions and the 10 -item Cons scale correlates .92 with the Cons-P scale, so little information is lost with this scale. (Appendix C presents external validity evidence for the 10-item Cons scale.) The investigation of the Cons-A scale will provide additional 
information as to the nature of the Cons of immoderate drinking for college students. Scale scores were calculated for each subject who answered to at least $50 \%$ of the scale items by taking the unweighted mean of item responses. Means, standard deviations, alphas and scale intercorrelations for both samples are presented in Table 4-3.

\section{EXTERNAL VALIDITY}

The relationship of the three Decisional Balance scales to several variables selected to provide external validity were investigated. The variables examined included age and gender, three alcohol consumption variables, three scales measuring negative alcohol-related experiences, and two measures of the Stage of Change construct of the Transtheoretical Model for immoderate alcohol use. Pearson-r correlations were calculated for the continuous variables and analysis of variance techniques were used for the categorical variables of gender and Stage of Change. Correlations are presented in Table 4-4 and will be discussed first.

All three Decisional Balance scales had moderate negative correlations with age. This suggests that as students progress through college the Pros and Cons of drinking become less important to them. This might represent a reduction in the salience of the Pros and Cons as students settle into a more stable and comfortable pattern of drinking or alternatively a movement from an extremity to centrality response bias.

The correlations of the Decisional Balance scales to the three consumption variables, Days, Drinks, and Intoxication, exhibited a consistent pattern of positive correlations ranging from .38 to .15 with the Pros scale and small negative correlations with the two Cons scales, ranging from -.11 to -.03 . These results suggest that those who value the positive aspects of drinking are more likely to drink more and have higher tolerance to alcohol than those who did not. Additionally this relationship is stronger than the inverse, namely the negative relationship between drinking and the evaluation of two types of Cons of drinking, which suggests that the Pros are more central to decisions about alcohol consumption. 
The Pros are positively related to all three Alcohol-Related Experiences scales, with especially strong correlations with the Excess and Distress scales $(r=.42$ and $r=.43$, respectively). This suggests that those who value the advantages of drinking are likely to experience the negative aspects of drinking. This relationship is probably mediated by positive association between the Pros and levels of consumption as delineated above and suggests that the Pros of drinking outweigh the actual experience of negative consequences of drinking in decision making.

The Cons-P scale had smaller negative correlations with these three scales. The correlation with the Excess scale was the largest at -.13 and suggests that those who recognize the risks inherent in excessive episodes of drinking experience fewer consequences of excessive drinking. This is a small effect and might be somewhat mediated be the negative relationship between the Cons-P and consumption.

The Cons-A had a different relationship with Alcohol-Related Experience scales than the Cons-P. This scale has a non-significant correlation with the Excess scale, a moderate positive correlation with the Distress scale $(r=.25)$, and a small positive correlation with the Problem scale $(r=.11)$. These positive correlations suggest that those who suffer these types of experiences evaluate the present emotional consequences of drinking as important in their decision making about their drinking.

\section{Relationship of Decisional Balance with the Stages of Cessation}

The relationship between gender, Stage of Change, and the Decisional Balance scales were investigated. Two measures of Stage of Change, called Algorithmic Stage and Adjusted Clusters were used in separate analysis. The analysis using the algorithmic measure is reported first.

Algorithmic Stage by gender ANOVAs were run for all three scales. In none of these analyses was gender or the Stage by gender interaction a significant effect. The Stage effect for the Pros was significant $\left(\underline{\mathrm{F}}(4,409)=14.96, \underline{\mathrm{p}}<.0001, \omega^{2}=.12\right)$. Follow-up Tukey tests 
showed that subjects in the Precontemplation stage evaluated the Pros of drinking significantiy more positively than those in Action or Maintenance, as did those in Contemplation and Preparation as compared to those in Maintenance. The stage effect for the Cons-P scale was not significant $(\mathrm{F}(4,408)=.60, \mathrm{p}>.05)$ but was significant for the Cons-A scale $\left(\mathrm{F}(4,409)=3.60, \mathrm{p}<.0 .01, \omega^{2}=.024\right)$. Follow-up Tukey tests showed that Precontemplators scored significantly lower on the Cons-A than either Preparers or Maintainers. Scale means and standard deviations by Stage are presented in Table 4-5. Additionally, scale scores for these three scales were converted to $T$-scores $(\underline{M}=50, \underline{S D}=10)$ and presented by Stage in Figure 4-4.

From Figure 4-4 the generally linearly decreasing trend in the Pros can be seen, as can the generally flat pattern for the Cons-P, with some elevation of the value for Maintainers. The pattern for the Cons-A scale shows a high elevation at Preparation and slightly lower one at Maintenance. The unusual aspect of this pattern is the low score for those in Action. Although previous research has found that in cessation the Cons decrease in the later stages, this trend is usually not as pronounced and continues into Maintenance (Prochaska et al., 1994). To further investigate this phenomenon, subjects who were also in a cluster that was not clearly associated with a primary stage were removed from the Action group. The stage means were recalculated for both Cons scales and plotted in Figure 4-4. This increases the scale mean for the Action group and smooths the pattern across the stages.

An important aspect of Figure 4-4 is the position of the crossover between the Pros and each of the Cons. This crossover can be considered the point that the balance of the Pros and Cons changes and begins to favor a decision to change the behavior. For both Con scales the crossover is between the Contemplation and Action stages. This is consistent with what has been found in previous research (Prochaska et al., 1994). Previous research has also discovered quantitative relationships between Decisional Balance scales and Stage of Change (Prochaska, 1994). These relationships predict a maximum $10 \mathrm{~T}$-point change in the Cons across the stages and a 5 T-point change in the Pros. It was found instead that the Pros 
changed 9.2 T-points and the Cons-A changed 6.7, approximately the reverse of what would be predicted. This suggests that the Pros are more related to stage progression than are the Cons for this behavior. Also these data suggest that the experienced emotional sequelae of drinking are more related to stage status than is the assessment of risk incurred by drinking immoderately.

Adjusted Cluster by gender ANOVAs were also conducted. Similarly in these analyses the main effect of gender and the interaction proved to be non-significant. The main effect of Adjusted Cluster was significant for the Pros scale $(\underline{F}(4,324)=9.31, p<.0001$, $\omega^{2}=.092$ ). Follow-up Tukey tests showed that those in Precontemplation and Contemplation valued the Pros of drinking significantly more than those in Action or Maintenance, as did those in Preparation compared to Maintenance.

The main effect of both of the Cons scales were significant (Cons-P: $(\underline{F}(4,323)=5.33$, $\mathrm{p}<.0 .001, \omega=.062)$; Cons-A: $\left.\left(\mathrm{F}(4,324)=16.63, \mathrm{p}<.0001, \omega^{2}=.16\right)\right)$. Follow-up Tukey tests showed that Precontemplators rate the Cons-P as significantly less important than those in Action or Maintenance. On the Cons-A scale those in Precontemplation scored lower than all four other stages. Also, those in Contemplation scored lower than those in Action. Means and standard deviations for the three scales by Adjusted Cluster are presented in Table 4-6.

Figure 4-5 shows that, as T-Scores, the values of the Pros and Cons crossover close to Contemplation for both scales, which is consistent with previous research. Furthermore, the Cons-A scale peaks at Action in stark contrast to the pattern across the Algorithmic Stages. The Cons-A exhibited a difference of $11.8 \mathrm{~T}$-points, close to the predicted value. The Cons-P only showed a 6.7 T-points difference, somewhat less than predicted. On the other hand the Pros showed a 9.5 T-points difference, almost twice predicted, added evidence that the Pros might be a more important variable in college drinking than it has been for other cessation behaviors. 
An set of additional data points was included in these Figure 4-5. The values for the Decisional Balance scales were plotted for the Immotive-M cluster. This cluster did not clearly correspond to one of the five Stages of Change, and had a scale profile characterized by an average score on the Precontemplation scale, a low score on the Contemplation scale, and a high scores of the Maintenance scale (see Study II for details). This group's values on the Decisional Balance scales suggest it is not a subgroup of one of the other stages.

Although its Pros value is similar to late stage subjects, its scores on the Cons-P and Cons-A scales are even lower than Precontemplators. One hypothesis is that this cluster represents a group of students who have moderated their drinking as a consequence of changing environmental factors with little intentional effort. Decisional Balance scales might have little salience for those who have not consciously struggled with their drinking habits. 


\section{DISCUSSION}

These results support the use of the Decisional Balance for College Drinking instrument to measure the Pros and Cons for this population and show that this construct of the Transtheoretical Model is applicable to immoderate college drinking largely as hypothesized. The results suggest that the scales are psychometrically sound with good confirmatory model fit indices and internal reliability, and high item saturation. The internal reliability of the Cons-A scale could be improved by the generation of additional items.

These results also suggest that college students recognize two classes of negative results of drinking: those associated with emotional sequelae of drinking and those associated with the risk of more concrete or practical harm. This is a departure from most previous research which has found a uni-dimensional Cons component. This dichotomy has some resemblance to the utilitarian/approval distinction of Janis and Mann's theory, although the items that load on the Cons-A scale do not obviously measure disapproval.

The decision of whether to pursue the two or three factor model was not strongly indicated by the empirical findings. The improvement in model fit of the three factor model was minimal, and it could be argued that it was insufficient to support a model that departs from the large body of previous research compiled on this construct, albeit with other behaviors. However, it was decided to pursue the three factor model because it was felt that little information was lost since the Cons-P and full Cons scale correlate so highly and it allowed an exploratory investigation of the Cons-A scale. Furthermore, as suggested by the Hierarchical Model, the three factor model does not conflict with a two dimensional Decisional Balance model, but is an elaboration of it. The differences between these two scales on external validity variables supports the utility of the three factor model. Further research will be needed to decide which model replicates and is most useful for this behavioral domain. 
This research also provided strong external validity evidence for the Decisional Balance scales for college drinking. The scales have strong correlations with a number of validating variables. This was especially true for the Pros scale which had high correlations with the consumption variables and all three Alcohol-Related Experience scales. This is the first piece of evidence that the Pros are more strongly related to college drinking than are the Cons which had weaker correlations to all the validity scales.

Of special interest to this investigation are the relationships of these scales to the Alcohol-Related Experience scales since it is these experiences that represent the main reasons that immoderate drinking is immoderate (i.e. unhealthy, unwise). Although it might be hypothesized that the experience of problems from drinking would lead to a lower endorsement of the Pros of drinking, the correlation observed is positive. This suggests the opposite direction of causation, namely that higher Pros lead to higher levels of drinking problems, and this relationship is probably mediated by consumption levels. It also suggests that if experiencing the problems of drinking leads to a lowering of the Pros, it does not do so immediately, but only after a delay.

The relationship of the Cons scales to the Alcohol-Related Experience scales is more complicated. The Excess scale's small negative correlation with the Cons-P scale is likely mediated somewhat by consumption levels. Those who do take the risks of immoderate drinking seriously (have high Cons-P scores) are likely to act in ways to avoid these consequences of drinking. Why this relationship is so attenuated for the Distress and Problem scales might be related to the nature of these scales. The lower endorsement of these scales might reflect that these experiences occur only to more vulnerable persons. Alternatively, perhaps both the weakness of these correlations and the positive correlations between the Cons-A and the Distress and Problem scales could be explained by a reversal in the direction of causation. It might be that the experience of these types of negative sequelae of drinking leads to an increase in the appreciation of the Cons of drinking. The direction of causation 
can not be determined from correlational data and confirmation of these hypotheses will await longitudinal research.

The relationship between the Decisional Balance scales and the Stages of Change show a general pattern that is as expected, with decreasing Pros and increasing Cons with stage progression with both methods of classifying Stage of Change. These data also allow a comparison of two different staging techniques, an important model construct. The hypothesis that the Adjusted Clusters represent purer stage groups is supported. The Decisional Balance patterns of the Decisional Balance scales for the Adjusted Clusters are smoother, closer to predictions and this classification explains greater amounts of the variance for the Cons-P and Cons-A scales than do the Algorithmic Stages. The disadvantages, as discussed in Study II, are the number of subjects who are unstaged, and the more difficult task of assigning a Stage of Change.

The anomalous reduction in the Cons-A scale for the Action algorithmic stage is of interest. It can be hypothesized that some students stop drinking immoderately without much effort because their drinking habits are in flux, with the process of acquiring the habit alternating or co-occurring with the process of giving it up, and with environmental factors playing a large role. It might be that using a behavioral criterion for Action includes some who stopped drinking immoderately because of a 'failure' to acquire the habit (are acquisition relapsers) and therefore do not have the predicted attitudinal set. The Adjusted Clusters avoid this problem by using attitudinal measures to classify subjects. This hypothesis is similar to the explanation of the URICA-A scales profile of the Immotive-M group (see Study II), and $26 \%$ of the subjects in the algorithmic Action stage are cross-classified into this cluster. Removal of these subjects smooths the graphs and lends support to this hypothesis. Additionally the co-occurrence of acquisition and cessation might also explain the stronger than predicted relationship of the Pros to Stage, which can be conceptualized as the Cons of drinking acquisition. 
It is also of interest that the Cons-P scale has weaker external validity evidence than the Cons-A. The Cons-P is closer to measuring the salience of the major acute risks of immoderate drinking, and this finding suggests that students' assessments of risk have less impact on them than does their actual experience. This would be consistent with the common observation that adolescents and young adults have a different relationship with risk than do older adults and are seen as being more likely to be risk seeking and risk ignoring.

These findings suggest that interventions designed to change college students" assessments of the Pros and the Cons might be effective in promoting positive stage change. Furthermore, these data, if found to be robust, could form the basis of stage matched interventions. It is thought that matching interventions shows more promise than the generally unsuccessful whole group interventions that have been traditionally attempted. It has been shown with smokers that an intervention matched by Stage and intervening on using other Transtheoretical Model constructs including Decisional Balance is more effective than other approaches (Prochaska, DiClemente, Velicer, \& Rossi, 1993).

The patterns across the Adjusted Clusters suggest that early stage movement is more related to the Cons scales, whereas late stage movement shows large changes in both the Cons and Pros scores. The strong relation of the Pros to Stage argues for approaches that acknowledge how students use alcohol to make their lives better and help them find other ways of accomplishing the same results without immoderate drinking. The reduction in the Pros between Action and Maintenance suggests that this approach might be especially useful in helping students maintain reduced drinking, which has been a common failure of interventions with college students. Intervening on the Cons-A might be easier and more effective than on the Cons-P variable. The risks assessed in the Cons-P are well known and often included in the content of the usual drug education programs whereas college students might be relatively unaware of the more emotional effects of drinking. Simple education might be effective. 
This study was an initial attempt to construct scales that accurately measure the Decisional Balance construct for drinking in college and provide basic validity evidence for this instrument. The success of this endeavor, along with the questions that these results raise, supports the importance of further research on the Decisional Balance construct. Besides additional work on the development of a fuller Cons-A scale as has been previously suggested, further research should include replication of these results on more representative samples across a range of colleges. Longitudinal research would help answer a number of questions including whether the patterns seen cross-sectionally across Stage actually represent how students change as they progress through stages. Although this has been generally found with other behaviors, it needs to be demonstrated for this complicated behavior and dynamic population. Such research could also provide evidence for the predictive utility of these scales. Lastly, intervention research that attempts to change Decisional Balance attitudes would help determine the utility of the Decisional Balance construct for reducing the harm that immoderate drinking causes in college populations. 


\section{REFERENCES}

Allen, S. J. \& Hubbard, R. (1986). Regression equations for the latent roots of random data correlation matrices with unities on the diagonal. Multivariate Behavioral Research, $21,393-398$.

Bentler, P. M. (1989). EQS:Structural equations program manual. Los Angeles, California: BMDP Statistical Software.

Brown, S. A. (1985). Expectancies versus background in the prediction of college drinking patterns. Journal of Consulting and Clinical Psychology, 53, 123-130.

Brown, S. A., Goldman, M. S., Inn, A., \& Anderson, L. R. (1980). Expectations of reinforcement from alcohol: Their domain and relation to drinking patterns. Journal of Consulting and Clinical Psychology, 48, 419-426.

Cattell, R. B. (1966). The Scree test for the number of factors. Multivariate Behavioral Research, 1, 245-276.

DiClemente, C. C., Prochaska, J. O., Fairhurst, S. K., Velicer, W. F., Velasquez, M. M., \& Rossi, J. S. (1991). The process of smoking cessation: An analysis of precontemplation, contemplation and preparation stages of change. Journal of Consulting and Clinical Psychology, 59, 295-304.

Horn, J. L. (1965). A rationale and test for the number of factors in factor analysis. Psychometrika, $\underline{30}, 179-185$.

Janis, I. L. \& Mann, L. (1968). A conflict-theory approach to attitude change and decision making. In A. Greenwald, T. Brook, \& T. Ostrom (Eds.), Psychological foundation of attitudes (pp. 327-360). New York: Academic Press.

Janis, I. L. \& Mann, L. (1977). Decision making: A psychological analysis of conflict. choice and commitment. New York: Free Press.

Kraft, D. P. (1988). The prevention and treatment of alcohol problems on a college campus. Journal of Alcohol and Drug Education, 34, 37-51. 
Marlatt, G. A. \& Rohsenow, D. J. (1980). Cognitive processes in alcohol use: Expectancy and the balanced placebo design. In N. K. Mello (Ed.), Advances in substance abuse: Behavioral and biological research (pp, 159-199). Greenwich, CT: JAI Press.

Meilman, P. W., Stone, J. E., Gaylor, M. S., \& Turco, J. H. (1990). Alcohol consumption by college undergraduates: Current use and 10-year trends. Journal of Studies on Alcohol, 51, 389-395.

Migneault, J. P., Pallonen, U. E., \& Velicer, W. F. (1994, April). Stage and decisional balance for adolescent alcohol use. Poster submitted for presentation at the 15 th annual meeting of the Society of Behavioral Medicine, Boston, MA.

Migneault, J. P., Stevenson, J. F., \& Velicer, W. F. (1994, April). A validation study of the stages of change for risky drinking in college. Paper presented at the 15 th annual meeting of the Society of Behavioral Medicine in Boston, MA.

Prochaska, J. O. (1994). Strong and Weak Principles for Progressing from Precontemplation to Action Based on Twelve Problem Behaviors. Health Psychology, 13, 47-51.

Prochaska, J. O., DiClemente, C. C., Velicer, W. F., Ginpil, S., \& Norcross, J. C. (1985). Predicting change in smoking status for self-changers. Addictive Behaviors, $10,395-$ 406.

Prochaska, J. O., DiClemente, C. C., Velicer, W. F., \& Rossi, J. S. (1993). Standardized, individualized, interactive and personalized self-help programs for stages of smoking cessation. Health Psychology, 12, 399-405.

Prochaska, J. O., Velicer, W. F., Rossi, J. S., Goldstein, M. G., Marcus, B. H., Rakowski, W., Fiore, C., Harlow, L. L., Redding, C. A., Rosenbloom, D., \& Rossi, S. R. (1994). Stages of change and decisional balance for twelve problem behaviors. Health Psychology, $\underline{13}, 39-46$

Redding, C. A. (1993). The transtheoretical model applied to safer sex behavior among university students: A cross-sectional investigation. Unpublished doctoral dissertation, University of Rhode Island, Kingston, RI. 
Velicer, W. F. (1976). Determining the number of components from the matrix of partial correlations. Psychometrika, 41, 321-327.

Velicer, W. F., DiClemente, C. C., Prochaska, J. O., \& Brandenburg, N. (1985). A decisional balance measure for assessing and predicting smoking status. Journal of Personality and Social Psychology, 48, 1279-1289.

Zwick, W. R. \& Velicer, W. F. (1986). Comparison of five rules for determining the number of components to retain. Psychological Bulletin, $\underline{99}, 432-442$. 
Table 4-1: Principal component loadings for exploratory analysis of Decisional Balance items - Two component solution

Scale 1: $\underline{\text { Pros }}$

1 - I am more self confident when I drink.

2 - Drinking gives me more courage.

3 - I can talk with someone I am attracted to better after a few drinks.

4 - Drinking makes me more relaxed and less tense.

5 - I feel happier when I drink.

6 - Drinking helps keep my mind off problems.

7 - Drinking helps me have fun with friends.

8 - Drinking makes me feel more independent.

9 - Drinking gives me a thrilling high feeling.

10 - When I drink my body feels better.

\section{Component}

I $\underline{\text { II }}$

.82

.81

.81

.80

.80

.79

.79

.74

.72

.69

\section{Scale 2: Cons}

1 - Drinking could kill me. $\quad .78$

2 - Drinking could get me addicted to alcohol. $\quad .75$

3 - Drinking could land me in trouble with the law.

4 - Drinking is bad for my health. $\quad .70$

5 - Drinking causes problems with others.

6 - I am setting a bad example for others with my drinking.

7 - I might end up hurting somebody.

8 - After a few drinks it is easier for others to take advantage of me. $\quad .59$

9 - I do not do as well at school because of my drinking.

10 - I do not like myself as much when I drink. 
Table 4-2: Principal component loadings for exploratory analysis of Decisional Balance items -

\section{Three component solution}

Scale 1: $\underline{\text { Pros }}$

1 - I can talk with someone I am attracted to better after a few drinks.

2 - I am more self confident when I drink.

3 - Drinking helps me have fun with friends.

4 - I feel happier when I drink.

5 - Drinking makes me more relaxed and less tense.

6 - Drinking gives me more courage.

7 - Drinking helps keep my mind off problems.

8 - Drinking gives me a thrilling high feeling.

9 - Drinking makes me feel more independent.

10 - When I drink my body feels better.
Component

I II III

.87

.84

.82

.82

.80

.79

.78

.70

.70

.63

\section{Scale 2: Cons-P}

1 - Drinking could kill me.

2 - Drinking could land me in trouble with the law.

3 - I might end up hurting somebody.

4 - Drinking is bad for my heaith.

5 - Drinking could get me addicted to alcohol.

\section{Scale 3: Cons-A}

1 - I do not like myself as much when I drink.

2 - Drinking makes me feel out of control.

3 - After drinking I often wake up feeling down. 
Table 4-3: Means, standard deviations, coefficient alphas and scale intercorrelations for the Decisional Balance scales for both samples

Sample $1(\mathrm{~N}=302)$

\begin{tabular}{lccccccc}
\hline & & & \multicolumn{5}{c}{ Correlations } \\
\cline { 7 - 8 } SCALE & Mean & SD & Alpha & Pros & Cons & Cons-P & Cons-A \\
\hline Pros & 2.76 & .91 & .93 & -- & & & \\
Cons & 3.08 & .94 & .86 & -.02 & -- & & \\
Cons-P & 3.45 & 1.11 & .84 & -.11 & $.91^{*}$ & -- & \\
Cons-A & 2.56 & 1.01 & .62 & $.18^{*}$ & $.57^{*}$ & $.36^{*}$ & -- \\
\hline
\end{tabular}

Sample $2(\mathrm{~N}=305)$

\begin{tabular}{lccccccc}
\hline & & & \multicolumn{4}{c}{ Correlations } \\
\cline { 6 - 7 } SCALE & Mean & SD & Alpha & Pros & Cons & Cons-P & Cons-A \\
\hline Pros & 2.83 & .87 & .91 & -- & & \\
Cons & 3.13 & 1.00 & .88 & $.20^{*}$ & -- & \\
Cons-P & 3.49 & 1.15 & .84 & $-.16^{*}$ & $.92^{*}$ & -- & \\
Cons-A & 2.66 & 1.09 & .72 & $.24^{*}$ & $.70^{*}$ & $.50^{*}$ & - \\
\hline
\end{tabular}

Note: * Correlation significant at $\mathrm{p}<.05$

Pros: 10-item scale common to the two and three factor solutions

Cons: 10 -item scale from two factor solution

Cons-P: 5-item Potential/Practical scale from three factor solution

Cons-A: 3-item Actual/Affectual scale from three factor solution 
Table 4-4: Correlation of Decisional Balance scales with extemal validity variables

\begin{tabular}{lccc}
\hline & Pros & Cons-P & Cons-A \\
\hline Age & $-.14^{*}$ & $-.12^{*}$ & $-.08^{*}$ \\
\hline
\end{tabular}

Alcohol Consumption

$\begin{array}{lccc}\text { DRINKS } & .38^{*} & -.10^{*} & -.03 \\ \text { DAYS } & .34^{*} & -.11^{*} & -.08 * \\ \text { INTOX } & .15 * & -.03 & -.07\end{array}$

Alcohol Related Experiences

$\begin{array}{lccc}\text { EXCESS } & .42^{*} & -.13^{*} & -.01 \\ \text { DISTRESS } & .43^{*} & -.02 & .25^{*} \\ \text { PROBLEM } & .20^{*} & -.07 & .11^{*}\end{array}$

Note: * Correlation significant at $\mathrm{p}<.05$ 
Table 4-5: Means and standard deviations for Decisional Balance scales by the Stages of Cessation

\begin{tabular}{|c|c|c|c|c|c|c|}
\hline \multirow[b]{3}{*}{ STAGE } & \multicolumn{6}{|c|}{ DECISIONAL BALANCE SCALES } \\
\hline & \multicolumn{2}{|c|}{ PROS } & \multicolumn{2}{|c|}{ Cons-P } & \multicolumn{2}{|c|}{ Cons-A } \\
\hline & Mean & $\mathrm{SD}$ & Mean & SD & Mean & SD \\
\hline Precontemplation & 3.16 & .69 & 3.37 & 1.06 & 2.54 & .94 \\
\hline Contemplation & 3.02 & .75 & 3.39 & 1.07 & 2.60 & .94 \\
\hline Preparation & 3.06 & .82 & 3.46 & .97 & 3.23 & .79 \\
\hline Action & 2.75 & .85 & 3.30 & 1.27 & 2.64 & 1.15 \\
\hline Maintenance & 2.42 & .87 & 3.60 & 1.18 & 2.91 & 1.22 \\
\hline
\end{tabular}


Table 4-6: Means and standard deviations for Decisional Balance scales by Adjusted Cluster

\begin{tabular}{|c|c|c|c|c|c|c|}
\hline \multirow[b]{3}{*}{ STAGE } & \multicolumn{6}{|c|}{ DECISIONAL BALANCE SCALES } \\
\hline & \multicolumn{2}{|c|}{ PROS } & \multicolumn{2}{|c|}{ Cons-P } & \multicolumn{2}{|c|}{ Cons-A } \\
\hline & Mean & $\mathrm{SD}$ & Mean & SD & Мean & SD \\
\hline Precontemplation & 3.19 & .73 & 3.25 & 1.05 & 2.35 & .88 \\
\hline Contemplation & 3.18 & .84 & 3.55 & 1.04 & 2.85 & 1.05 \\
\hline Preparation & 3.07 & .67 & 3.64 & 1.08 & 2.95 & .75 \\
\hline Action & 2.53 & .92 & 3.91 & 1.04 & 3.57 & 1.04 \\
\hline Maintenance & 2.42 & .90 & 3.99 & .87 & 3.24 & 1.12 \\
\hline
\end{tabular}


Figure 4-1: Two factor structural model for Decisional Balance scales

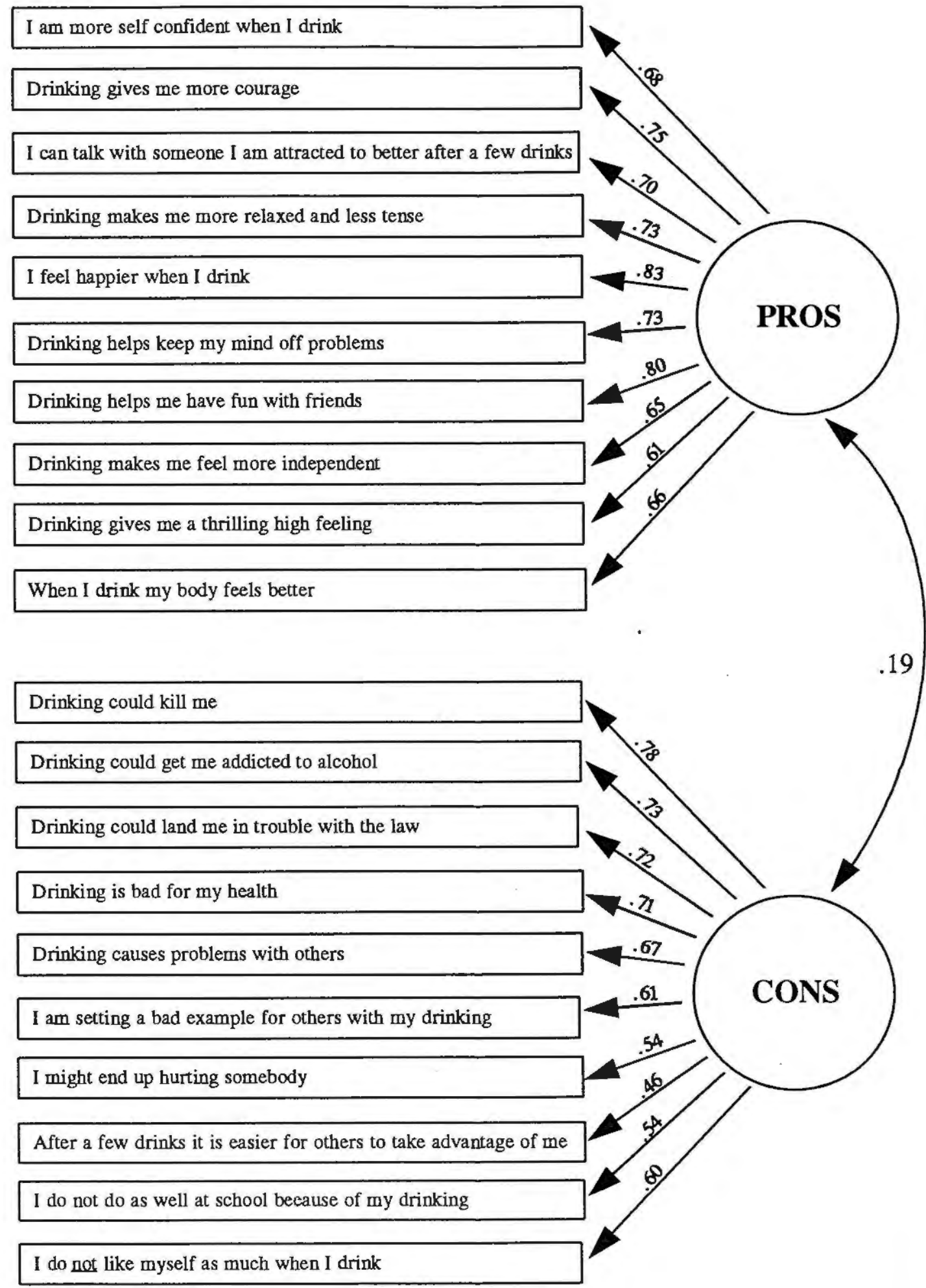


Figure 4-2: Three factor structural model for Decisional Balance scales

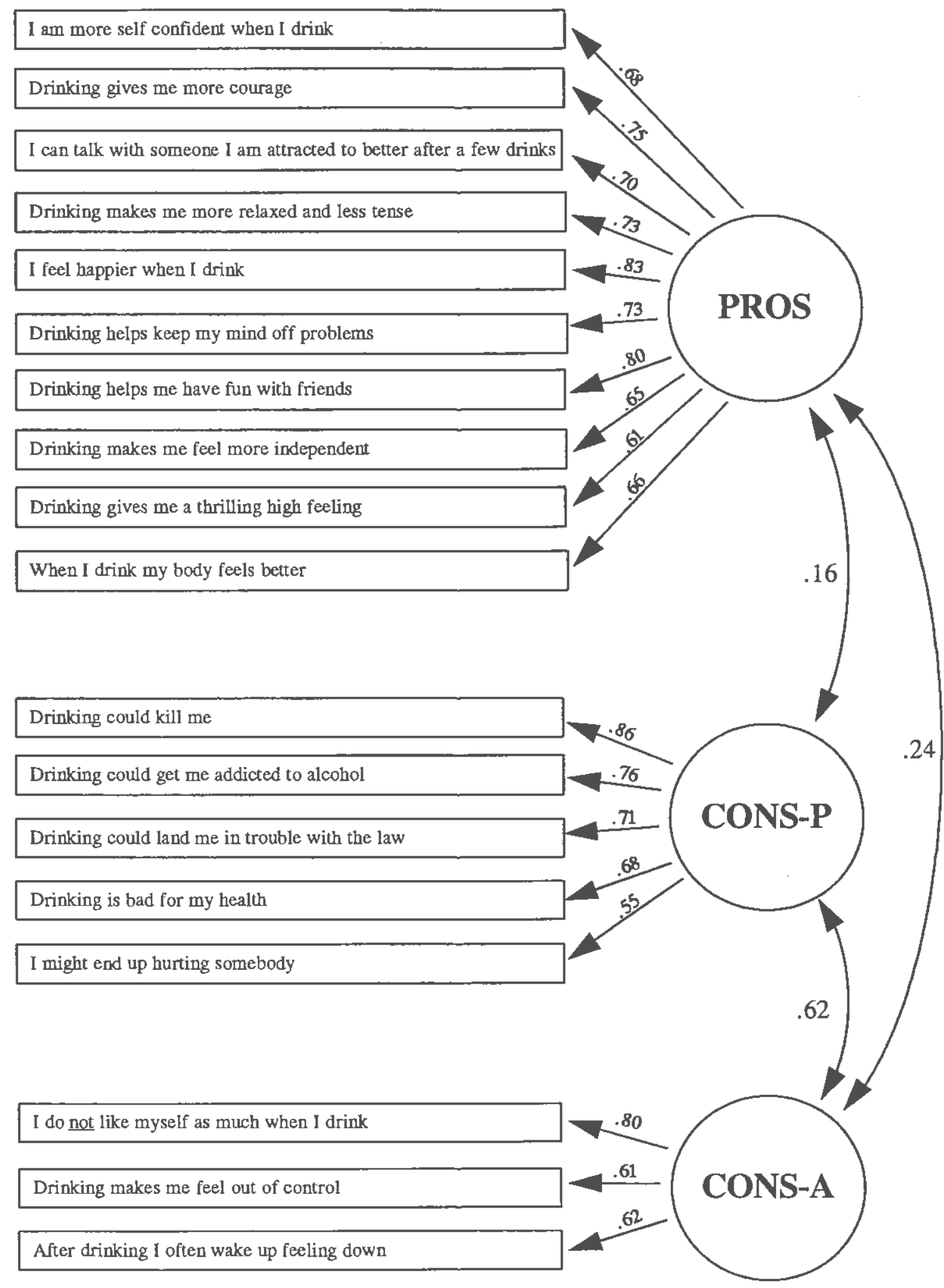


Figure 4-3: Hierarchical structural model for Decisional Balance scales

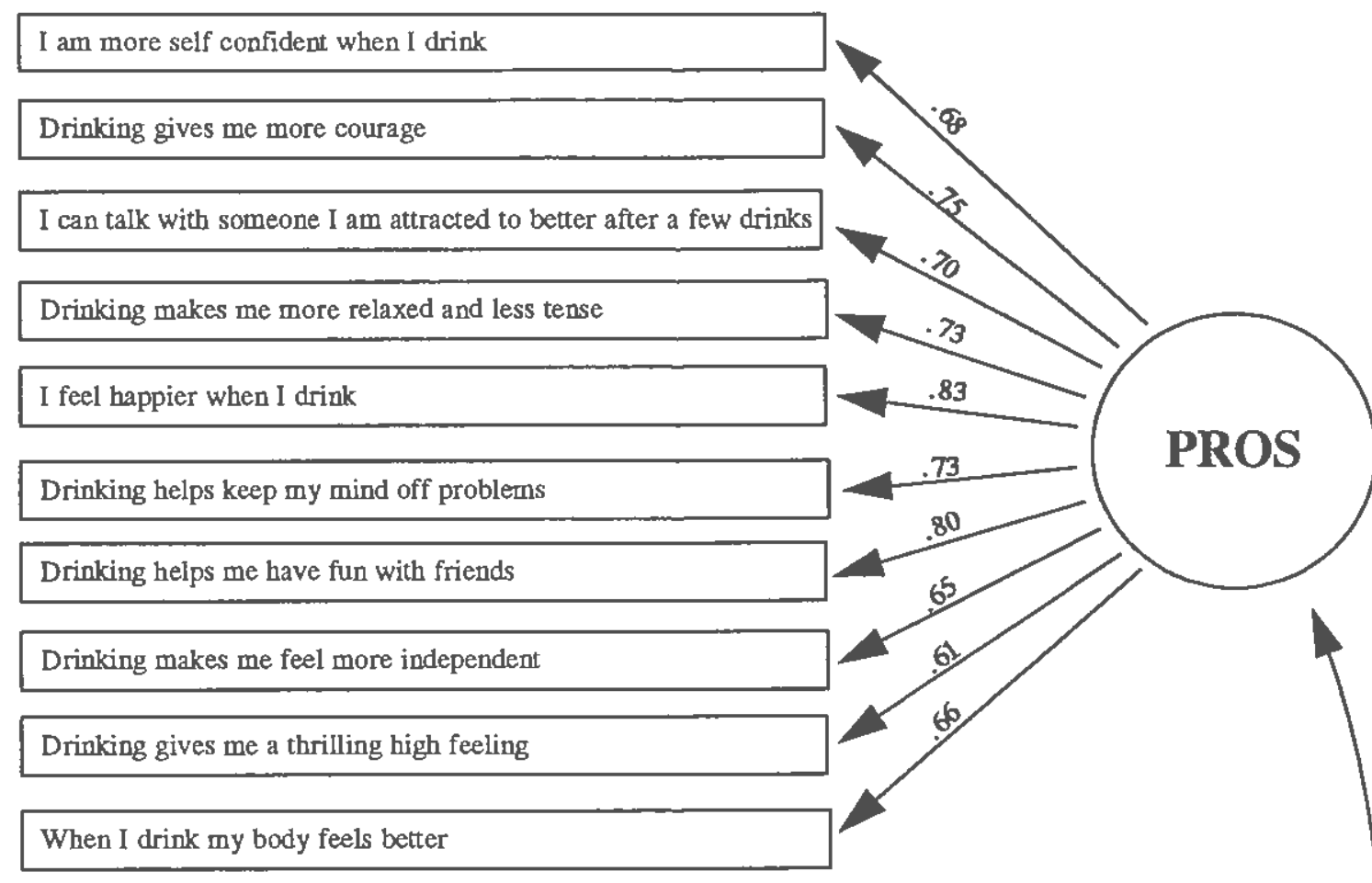

Drinking could kill me

Drinking could get me addicted to alcohol

Drinking could land me in trouble with the law

Drinking is bad for my health

I might end up hurting somebody
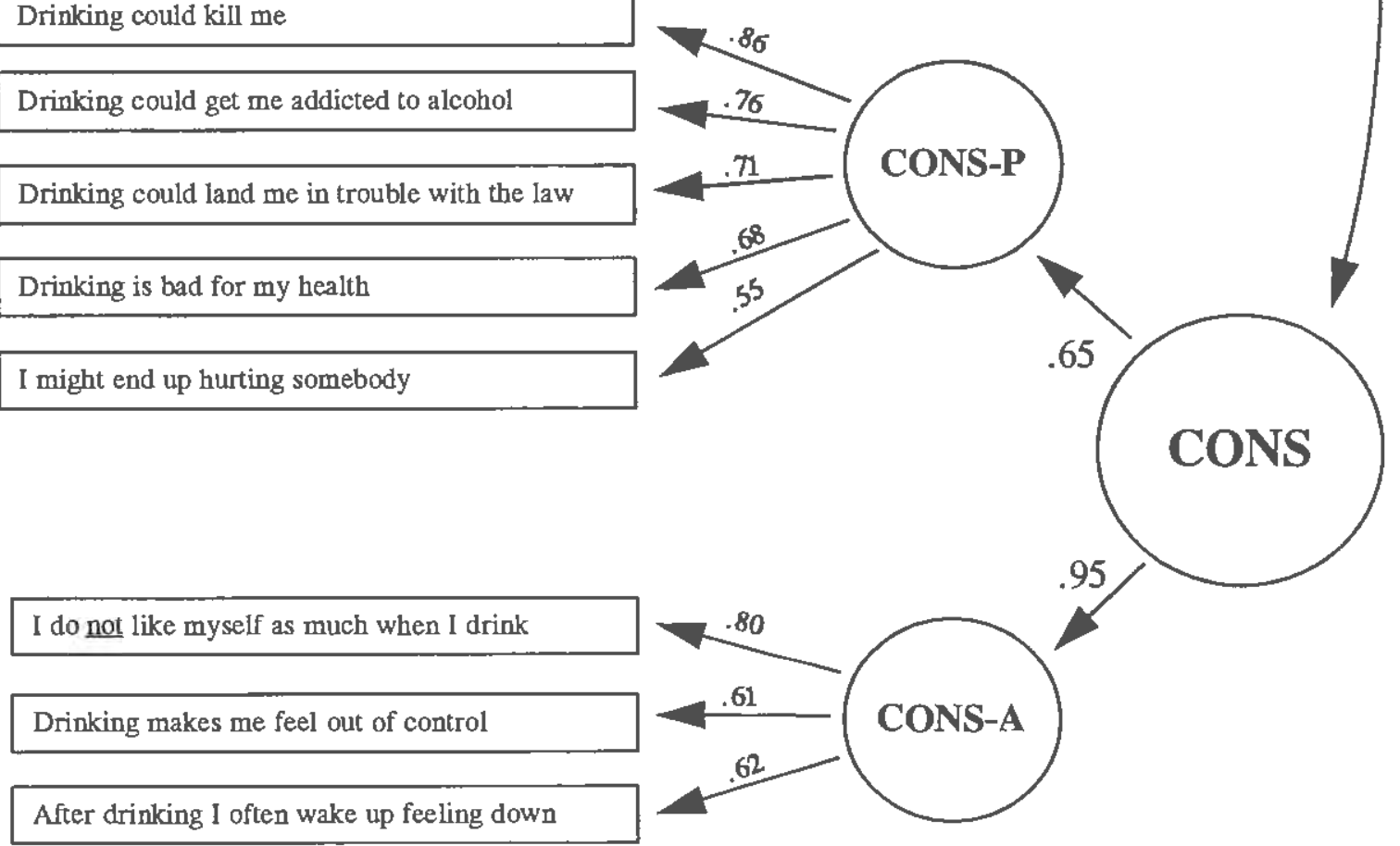
Figure 4-4: Decisional Balance scales by Stage of Change

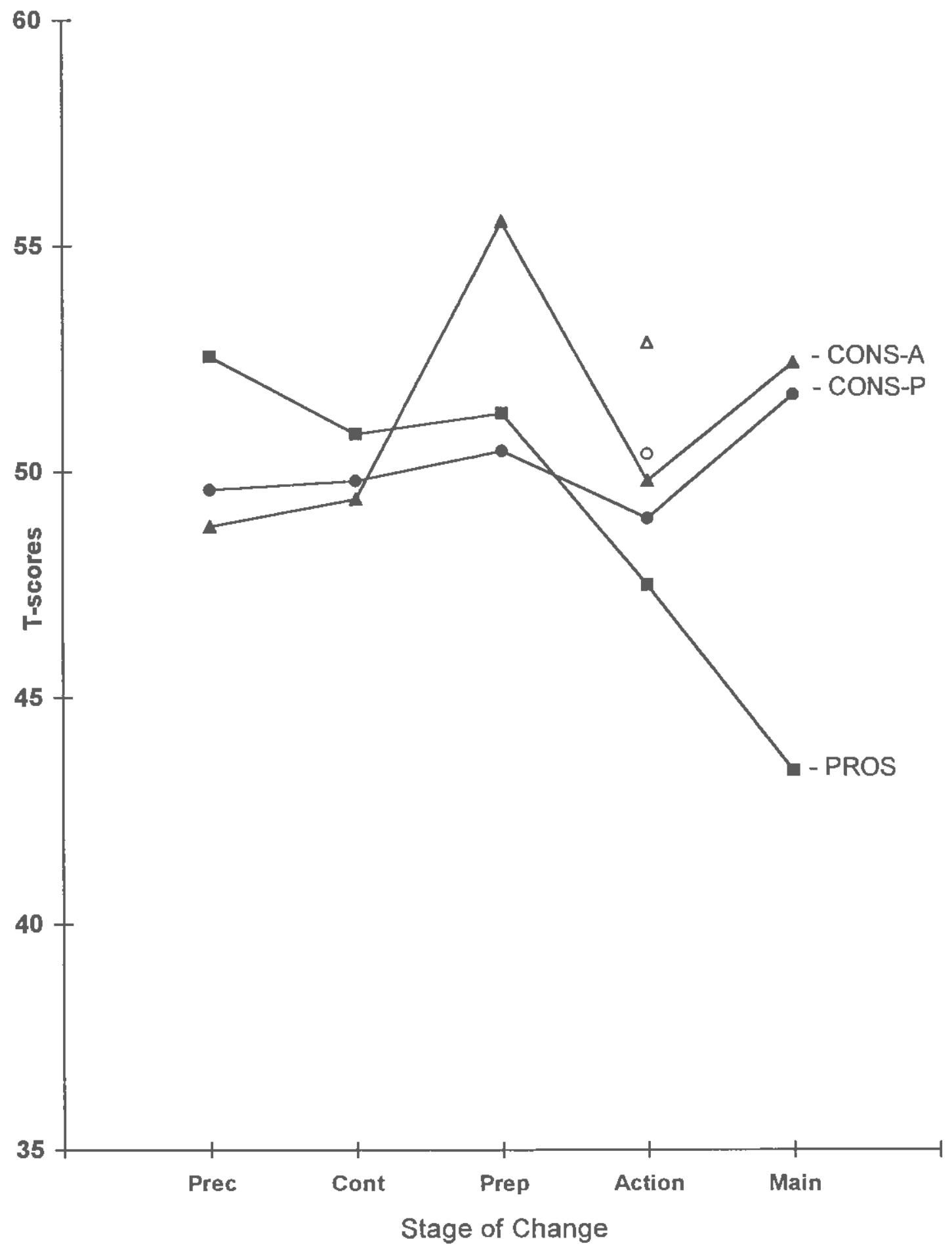

Note: Unconnected data points at Action are means with Immotive-M subjects removed 
Figure 4-5: Decisional Balance scales by Adiusted Cluster

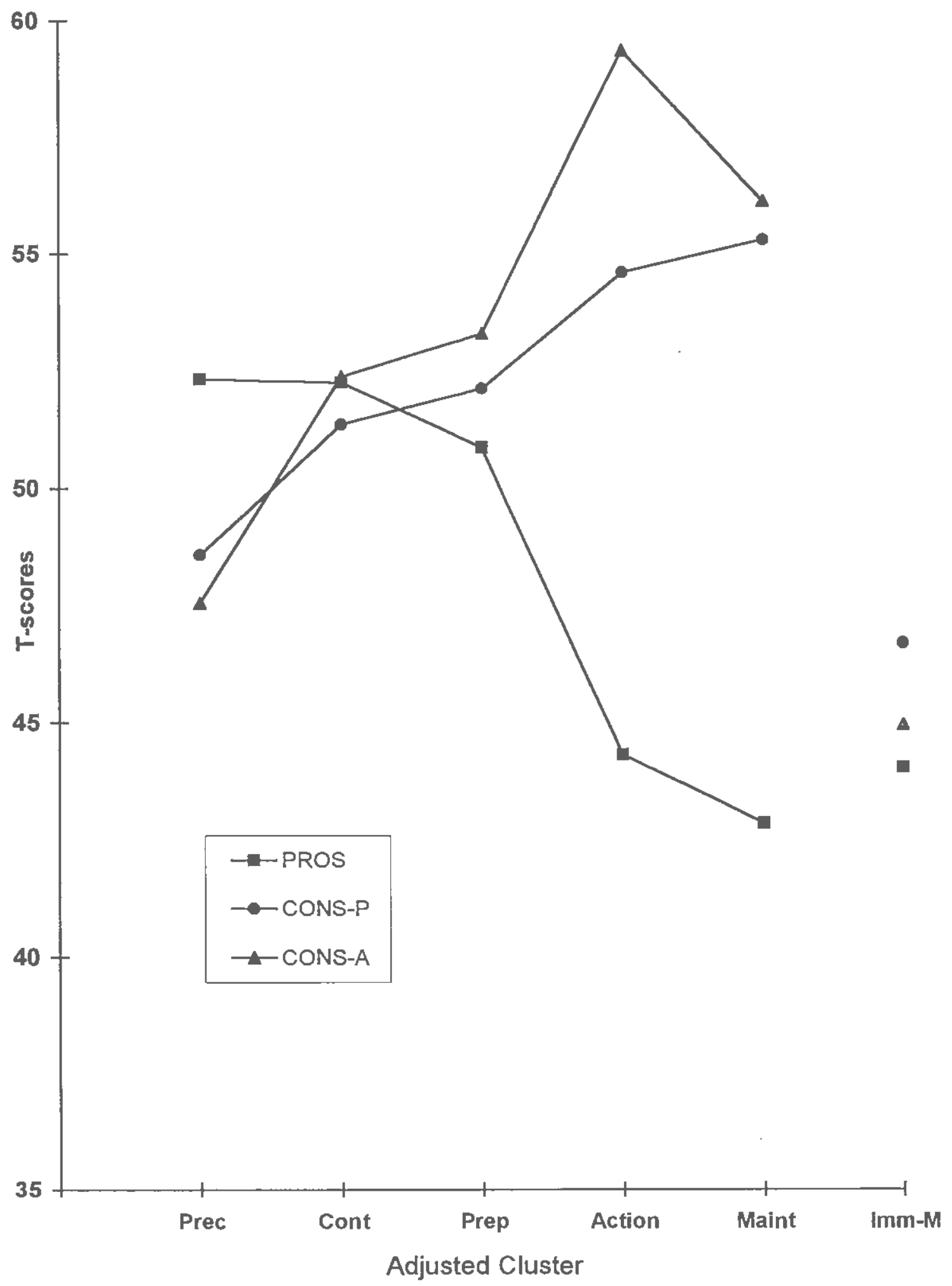


PART V. STUDY 4: TEMPTATIONS FOR IMMODERATE ALCOHOL USE

\section{INTRODUCTION}

A recent study observed that nearly half of a large national sample of students $(\mathrm{N}=17,592)$ were binge drinkers and that these students experienced high rates of drinkingrelated problems (Wechsler, Davenport, Dowdall, Moeykens, \& Castillo, 1994). The fact that students drink, despite the possible consequences, consequences that are continually presented in drug education programs, suggests that drinking habits are maintained by strong

forces. Yet from another point of view most college students do change their drinking habits, if not during college shortly thereafter, suggesting that these forces change or can be overcome.

Social Learning Theory posits both capabilities to change behavior and the forces that maintain them (Abrams \& Niaura, 1987; Perry, Baranowski, \& Parcel, 1990). A central tenet is Bandura's theory of Self-Efficacy (Bandura, 1977) which states that an individual's behavior will be strongly influenced by their perceptions of how capable they are of a specified behavior. This theory has received strong empirical support (Bandura, 1982).

Also integrated into Social Learning Theory is the notion of cue strength or temptation. This is the felt urge to engage in a behavior when exposed to certain environmental or internal stimuli. It is theorized that temptation is a function of a number of processes including classical and operant conditioning, and cognitive variables such as learning and expectancies. This complex theory suggests that the balance of self-efficacy and temptation will be highly predictive of behavior. Furthermore one's assessment of selfefficacy will be heavily influenced by one's assessment of the strength of the urges to not behave in an efficacious manner.

The Transtheoretical Model of Change is a multidimensional model of change that integrates a number of constructs including self-efficacy (DiClemente, 1986). Researchers 
using this model have developed instruments measuring self-efficacy for a wide range of behaviors (Clark, Abrams, Niaura, Eaton, \& Rossi, 1991; Marcus, Selby, Niaura, and Rossi, 1992; Prochaska, Harlow, Redding, Snow, Rossi, Velicer, et al., 1990; Redding 1993; Snow, 1991; Velicer, DiClemente, Rossi, \& Prochaska, 1990). These have taken two forms.

Confidence scales that assess the confidence or self-efficacy that a person has in not engaging in a certain behavior given specific situations or experiences and Temptation scales that assess the strength of temptation to engage in the behavior given the same set of situations. As predicted by theory these scales have been highly negatively correlated, and it has been seen only necessary to measure one aspect. For the behavior of substance use it is thought that the temptation items are more easily responded to by subjects (Velicer et al., 1990).

The Temptation instruments that have been developed have been behavior specific with items designed to tap situations that are tempting for a behavior and at times a specific population. Furthermore most of these instruments are composed of correlated subscales. These scales vary in both item content and the nature of their subscales. For adult smokers these subscales have been labeled Habit Strength, Negative Affect, and Positive/Social. In a study of vocational students who smoke, Pallonen found evidence of additional subscales of Loss of Control and Peer Pressure (unpublished data). Examples of other subscales include Partner Pressure and Substance Use for safe sex practices (Redding, 1993), and Food Availability, Social Pressure, and Physical Discomfort for weight control (Clark et al., 1991). Despite these differences in instrument content the relationships of these Temptations scales to outcome and other model variables have been very consistent.

These subscales have been observed as highly correlated yet distinct in recent studies. It has been found that hierarchical models that include a higher order Temptation factor on which the subscales load have explanatory and heuristic value. This allows for a calculation of an overall Temptation score as well as subscales scores.

This model can be seen as an integration of two models (Velicer et al., 1990). Bandura's self-efficacy model has been operationalized as one underlying construct that is 
operable across situations. Others have suggested that temptation is situation specific. An example of this type of model for alcohol use is the Inventory of Drinking Situations (IDS) (Cannon, Leeka, Patterson, \& Baker, 1990). This scale was based on Marlatt and Gordon's $(1980,1985)$ rational taxonomy of eight relapse determinants including negative emotional states, negative physical states, positive emotional states, testing personal control, urges and temptations, interpersonal conflict, social pressure, and positive social interactions. Recent analyses have found only three separate scales named negative affective states, positive affective states and social cues, and attempts to test one's control (Cannon, et al., 1990). The population studied were alcoholics at a veterans hospital. The hierarchical model developed within the context of the Transtheoretical Model suggests that there is validity in both the onefactor model and the multiple situations model.

Another construct of the Transtheoretical Model is the Stages of Change. This construct provides an organizing structure to investigate the other constructs of the model, including Temptations. There are five primary stages: Precontemplation (not considering changing the target behavior), Contemplation (intending to change in the next six months), Preparation (planning change in the next 30 days), Action (having changed in the last six months), and Maintenance (having changed the behavior more than six months ago). These stages have been validated in many different behavioral domains, and where longitudinal research has been done, have been highly predictive of future behavior change (Prochaska, DiClemente, Velicer, Ginpil, \& Norcross, 1985).

It has been found that Temptation scales have a consistent relationship with Stage. Temptation is high in the early stages and decreases in Action and Maintenance. Confidence forms a mirror image with Temptation (DiClemente, Prochaska, \& Gilbertini, 1985). It has also been suggested that the Temptation subscales are differentiated by the middle stages of change, more so than those in the end stages. Precontemplators tend to be highly tempted across situations and those in Maintenance tend to have low temptation in all situations. (Velicer et al., 1990). 
Previous attempts to apply the Self Efficacy construct of the Transtheoretical Model to alcohol consumption have studied populations of adult alcoholic drinkers. Snow (1991) studying sober alcoholics developed scales that were originally hypothesized to measure five subscales in both the Confidence and Temptation formats. These five included the three found with smokers and two subscales taken from the work of Marlatt and Gordon (1985) called testing personal control and situational cues. These last two were expected to be especially salient to a sample of late stage subjects dealing with relapse. This hypothesis was not supported, as one global factor was found in the data for both the Confidence and the Temptation instruments. Snow hypothesized that this result might be a result of the population that he studied as it was dominated by individuals in Maintenance. This group, as stated above, tends to exhibit an extremity response that is probably a valid reflection of their felt self-efficacy and would lead to the uni-dimensional result found.

The purpose of the present study is to extend the use of the Self-Efficacy construct of the Transtheoretical Model to immoderate alcohol use by college students by first developing a psychometrically sound Temptation for Immoderate Drinking instrument that is applicable to college populations. External validity evidence will then be examined as will the relationships of this variable to other Transtheoretical Model variables to provide evidence for the applicability of this model to this behavioral domain. 


\section{METHOD}

\section{SUBJECTS}

Subjects were 629 students surveyed at the University of Rhode Island in the fall of 1993 and spring of 1994 between the ages of 18 and 25 years and 11 months. Most of the subjects were recruited from undergraduate classes in the psychology department, although a variety of other classes were also sampled including physical education, pharmacology, nutrition, and sociology. A small percentage was recruited from the fraternities and the university health center.

The sample was $66.3 \%$ female, and $94 \%$ white. The distribution across the classes was $17.4 \%$ freshmen, $32.2 \%$ sophomores, $19.1 \%$ juniors, $26.2 \%$ seniors, and $5.3 \%$ either fifth-year or non-matriculated. The average age was 21.3 years. Of these students $47 \%$ were classified as in-state and $39 \%$ lived in dormitories, $10 \%$ lived in fraternities or sororities, and $51 \%$ lived off campus.

The use of alcohol in this sample was substantial. Using a criterion of at least one drinking occasion in the last 30 days, $92 \%$ were classified as active drinkers. These subjects drank on average 8.6 days a month and consumed on average 5.3 drinks per occasion. There were substantial differences in drinking by gender with drinking women averaging 7.3 days per month and 4.4 drinks per occasion. For men these figures were 11.0 days and 6.9 drinks. Drinking women reported an average maximum amount drunk on any occasion in the past month as 6.8 , and men as 12.1 .

\section{INSTRUMENTS}

The survey administered contained 282 questions, including, of specific interest to this study, the following item sets. The full survey instrument is presented in Appendix A. 


\section{Demographics}

A set of 22 items asked about basic demographic information and drinking history. Variables examined in this study include age, gender, number of days in the last month that alcohol was consumed (Days), the number of drinks consumed during a typical drinking occasion (Drinks), and the number of drinks consumed before subjects start to feel intoxicated (Intoxication).

\section{$\underline{\text { Temptation Item Set }}$}

A set of 24 items hypothesized to measure temptations to drink excessively across three situations was included in the survey. A preliminary study investigated 40 items that were either adapted from an item set developed by Snow (1991) or created using undergraduate informants as an aid. These 40 items were hypothesized to measure five correlated sub-scales: Negative Affect, Positive/Social, Situational Cues, Habit Strength, and Peer Pressure. Principal component analysis suggested that only three, Positive/Social, Negative Affect, and Peer Pressure were meaningful to this population. Items that exhibited good psychometrics were taken from this item set. New items hypothesized to measure other aspects of these three components were also generated. Eight items were chosen as measures of each of these three subscales for inclusion in the present study. Subjects were asked to rate the temptation they would feel to drink more than they should in each of the 24 situations using a 5-point Likert scale with $I=$ Not at all tempted and $5=$ Extremely tempted.

\section{Stages of Change}

Algorithmic method. A staging algorithm used answers from a branched set of five items to classify subjects into one of nine groups that represented the five Stages of Acquisition and the five Stages of Cessation with one group representing both the Maintenance stage of acquisition and the Precontemplation stage of cessation. In the present study only the Stages of Cessation were investigated $(\mathrm{N}=426)$. The algorithm used was 
developed and investigated in Study I and used a criterion of usually drinking 4 or more drinks at least once in a typical week for women and 5 or more for men. The development and investigation of this staging method is presented in Study $\mathrm{I}$.

Continuous measure. Three scales which compose the 21-item University of Rhode Island Change Assessment for Alcohol instrument (URICA-A) were used to measure stage related attitudes. These scales included a Precontemplation scale which measures acceptance of heavy drinking, a Contemplation scale which measures ambivalence about one's drinking habits and plans to change them, and a Maintenance scale which measures attitudes consistent with having reduced one's drinking and maintained them at more moderate levels. Scale alphas are .70 for the Precontemplation scale, .83 for the Contemplation scale, and .78 for the Maintenance scale,

\section{Decisional Balance Item Set}

A set of 18 items that measure the Decisional Balance construct of the Transtheoretical Model of Change was included. These items compose three scales. The Pros scale measures the importance of 10 benefits of drinking in subjects' decisions about alcohol consumption. There were two Cons scales, Cons-P and Cons-A. The Cons-P is a 5item scale that measures the importance of the risks of concrete or practical consequences of drinking in their decisions. The Cons-A is a 3-item scale which assess the importance of the emotional effects of drinking. These scales have internal consistencies as measured by Cronbach's Alpha of $.92, .84$ and .68 , respectively.

\section{Alcohol-Related Experiences}

Three scales, developed in Study I, that measure types of negative alcohol related experiences were included as a central validity variable. The scales are composed of 23 items developed from an original set of 31 items and are labeled Excess, Distress, and Problem. The three scales have moderate inter-scale correlations ranging from .37 and .44 and high 
internal consistencies as measured by Cronbach's Alpha ranging from .80 to .88 . The Excess scale is hypothesized to measure problems associated with short-term excessive or binge drinking. The Distress scale measures experiences of emotional or introspective distress, and the Problem scale measures consequences of alcohol consumption that are associated with long term problem drinking.

Subjects were asked to circle the number of times in the last 12 months that they had had each experience related to their alcohol consumption. The following 6-point response scale was provided: $0,1,2,3-5,6-9,10$ or more times.

\section{PROCEDURE}

The items used in this study were included within a larger survey that assessed additional aspects of drinking behaviors and attitudes. Informed consent was obtained and subjects were assured of the confidentiality of their responses (See Appendix B for a copy of the consent form). Surveys were completed during class time, or subjects took the survey home and returned it. Most, although not all, of those doing the survey on their own time received a small amount of class credit for completing the survey. A few students who completed the survey at the health services center were given a pen emblazoned with the university name in exchange for completing the survey, 


\section{RESULTS}

\section{EXPLORATORY COMPONENT ANALYSIS}

Subjects were eliminated from the analysis for two reasons. First, nine subjects who had missing data on more than two of the 24 items were eliminated. Second, 15 subjects who showed no variability in their answer sets were also eliminated. This response pattern, although perhaps valid, does not help in the exploration of the underlying structure of the item set and can inflate inter-item correlations (Jackson, 1970; Velicer et al., 1990). The final sample consisted of 598 subjects or $95.1 \%$ of the original sample. The sample was then randomly split into two subsamples.

An exploratory principal components analysis (PCA) was conducted on the $24 \times 24$ matrix of item intercorrelations from sample 1 generated using pair-wise deletion $(\underline{N}=295)$. The number of components to retain was determined by comparing the results of three procedures that have been shown to be valid predictors of the correct dimensionality of an item set (Zwick \& Velicer, 1986). Parallel Analysis (Horn 1968; Allen \& Hubbard, 1986) suggested three components, whereas the Minimum Average Partial (Velicer, 1976) suggested four components and the Scree procedure (Cattell, 1966) suggested extracting either one or four components. Both the three and four component solutions were investigated.

For both solutions orthogonal (varimax) and oblique (direct quartimin) rotations were performed. The oblique rotations were chosen as more interpretable. Items which had low loadings on all the components or high loading on more than one component were eliminated. The resulting three component item set and loading pattern was identical to the items and loading pattern for the three largest components of the four component solution. Since all four components had at least four high loading items and were interpretable the four component solution was chosen for further investigation. Items and factor loadings are presented in Tabie 5-1. 
The first component, Peer Pressure, consisted of all eight items hypothesized to measure the temptation to drink brought on by encouragement of others. It had a Cronbach's Coefficient Alpha for Sample 1 of .92. The second component consisted of five of the original eight items hypothesized to measure temptation to drink elicited by negative emotions. It was labeled Negative Affect and had an alpha of .91. The third component consisted of four of the original eight items hypothesized to measure temptation to drink brought on by pleasant emotions or by positive social situations. This scale was labeled Positive/Social and had an alpha of .88 . These three components closely matched the three hypothesized temptation constructs.

The fourth component was composed of three items hypothesized to measure negative affective situations and one measuring social temptations. All four of these items seem to be measuring social uncomfortableness, inhibition, or anxiety. The item hypothesized to measure a social situation asks about the temptation associated with "being with someone you are attracted to". These results suggest that this item taps the uncomfortableness that this situation entails for college students rather than more pleasant aspects. This scale was labeled Social Anxiety and had a coefficient alpha of .87 .

The four component solution suggests that college students are tempted to drink differentially by the negative affects elicited by intrapersonal distress vs. interpersonal uncomfortableness. This study provided no evidence of a similar differentiation between intrapersonal vs. interpersonal positive affects. Further scale development should attempt to more systematically generate items to measure temptation due to social anxiety to further explore the breadth of this construct.

The final item set contained 21 items across the four subscales. The inter-component correlations were moderate and ranged from .30 to .47 . The four components accounted for $66.2 \%$ of the variance in the original item set. 


\section{CONFIRMATORY FACTOR ANALYSIS}

Confirmatory factor analysis using EQS structural modeling program (Bentler, 1989) was performed using the hold out sample $(\underline{N}=303)$. Four models were run. The Null model posits 21 independent variables and is not expected to fit the data but generates a set of statistics which are used in the generation of model fit indices. The second, a Single Factor Model, had all 21 items loading on one general Temptation factor. The third was a Fully Correlated Model which had four intercorrelated factors corresponding to the four components found in the exploratory analysis. The fourth model was a Hierarchical Model, consisting of the four factors loading on a higher order general Temptation factor. A set of fit indices including Chi-square statistic, Comparative Fit Index (CFI), Non-Normed Fit Index (NNFI), Root Mean Square (RMS), and Incremental Fit Index, type 2 (IFI2) were calculated to assess model fit. Since the CFI, NNFI and IFI2 produced a similar pattern of results, only the IFI2 and the RMS are reported here.

The Single Factor Model exhibited poor model fit (IFI2 $=.65$, RMS $=.078$ ). Both the Correlated and Hierarchical Models fit the data well with the Correlated Model showing slightly better fit indices. The IFI2 was .90 and the RMS was .042 for the Correlated Model and .89 and .048 , respectively, for the Hierarchical Model. The RMS values are well below the generally accepted .06 criteria for a well fitting model whereas the values for the IFI2 are at the generally accepted value of .90 . It is also of note that models with long measurement scales, such as the Peer Pressure scale, generally have lower fit indices because of the large number of paths that are constrained to zero. The Correlated and Hierarchical models are presented in Figures 5-1 and 5-2.

Choosing between models with small statistical differences is best done on theoretical and heuristic grounds. The Hierarchical Model is preferred because it integrates two well researched conceptual models, the unitary self-efficacy model (Bandura, 1977, 1982) and the multiple tempting situations model (Marlatt \& Gordon, 1985). Furthermore it is the model 
that has proven useful in other behavioral domains (Clark et al., 1991; Marcus, et al., 1992; Redding, 1993; Velicer et al., 1990).

Item loadings were high: all loadings were above .64 with a mean of .78 . The primary factor loadings on the second-order general Temptation factor were also high ranging from .60 to .87 . Scale scores for each subscale and the overall Temptations scale were calculated for each subject who answered to at least $50 \%$ of the scale items by taking the unweighted mean of item responses. Scale means, standard deviations, alphas, and scale intercorrelations for both samples are presented in Table 5-2.

\section{EXTERNAL VALIDITY}

To assess external validity of the Temptation scales their empirical relationship to a number of variables was examined. Pearson correlations were calculated for the full Temptation scale and the four subscales with age, three alcohol consumption variables, three scales measuring alcohol-related experiences, and the three scales composing the Decisional Balance construct of the Transtheoretical Model. In addition, analysis of variance techniques were used to investigate the relationship of the Temptation scales to both gender and Stage of Change. This set of variables provides information on the relationship of the Temptation scales to an important demographic variable for a still maturing population (age), three variables that are measures of the behavior being examined, three measures of the risks associated with this behavior, and a set of variables (Stage and Decisional Balance scales) that will assess the validity of the application of the Transtheoretical model to this behavioral domain.

The only significant correlation with age was a small negative correlation with the Negative Affect subscale (see Table 5-3 for all reported correlations). This result suggests that there is some tendency for older students to be less tempted to drink when they are experiencing unpleasant emotional states. 
In contrast the correlations between the alcohol consumption variables and the Temptation scales were generally moderate to large in magnitude, and all were significant at the .01 level. The full Temptation scale correlated from .24 to .51 with these three variables. The correlations with the four subscales ranged from .12 to .48 . These alcohol consumption variables were more strongly associated with the Peer Pressure and Positive/Social subscales, and least strongly with the Negative Affect subscale. This pattern suggests that consumption of alcohol for college students is most related to interpersonal influences. It is also worth noting that the magnitude of the correlations with the Intox variable is about half the magnitude of the other two consumption variables. This result might be due to the smaller range of this variable or the fact that this variable is more indirectly related to consumption than the other two variables.

The correlations of the three Alcohol-Related Experiences scales with the full Temptation scale were moderate to large, ranging from .33 to .59 , suggesting a clear association between temptation to drink across situations and the experience of negative sequelae of drinking. The pattern of correlations for the subscales showed that the Excess scale was most related to with the Peer Pressure and Positive/Social scales, and reflects the social nature of binge drinking in college.

For the other two Alcohol-Related Experiences scales, Distress and Problem, the correlations were nearly equal across the Temptation subscales with the Positive/Social scale having the lowest correlations, suggesting that these two scales are relatively more related to negative emotional states, both intra- and interpersonal than the Excess scale. The relatively lower correlations for the Problem scale might be an artifact of this scale's restricted range.

The correlations between the Temptation scales and the Decisional Balance scales are also presented in Table 5-3. The correlations with the Pros are very high: .75 with the full Temptation scale and ranging from .56 to .73 for the four subscales. These results suggest that those who are most likely to evaluate the Pros of drinking as important are also those 
who are most likely to be tempted to drink in all four situations assessed in this study, and especially in anxiety producing social situations.

The results for the Con scales were quite different, with no significant correlations found with the Cons-P scale. The Cons-A scale had a positive correlations with the Temptation scale and three of the subscales ranging from .11 to .24 . The correlation with the Positive/Social subscale was near zero. This is consistent with the nature of these scales, suggesting that those who experience pleasure in the positive, social aspects of drinking are not likely to experience negative emotional aspects of drinking as important.

The relationship between the Temptation scales and the Stage of Change variable was also examined. The full Temptation scale and the four sub-scales were entered into a twoway Stage by gender MANOVA. The main effect of Stage proved significant $(\Lambda(20,13344.18)=.792, \mathrm{p}<.0001)$, explaining $21 \%$ of the variance. The main effect of gender and the interaction of Stage and gender did not prove significant (Gender:

$(\Lambda(5,405)=.998, \mathrm{p}>.05)$; Stage by gender $(\Lambda(20,1344.18)=.955, \mathrm{p}>.05))$. Follow-up oneway ANOVAs were done on all five scales across the Stages of Change, and all were highly significant (Temptation: $\left(\mathrm{F}(4,414)=22.05, \mathrm{p}<.0001, \omega^{2}=.17\right)$; Peer Pressure: $\left(\mathrm{F}(4,414)=18.87, \mathrm{~g}<.0001, \omega^{2}=.14\right) ;$ Negative Affect: $\left(\mathrm{F}(4,414)=6.70, \underline{\mathrm{g}}<.0001, \omega^{2}=.05\right)$; Positive/Social: $\left(\mathrm{F}(4,414)=17.77, \mathrm{p}<.0001, \omega^{2}=.14\right)$; Social Anxiety: $(\underline{F}(4,414)=14.93$, $\left.\mathrm{g}<.0001, \omega^{2}=.12\right)$ ). Follow-up Tukey tests were then conducted to determine which pairwise comparisons were significant. Means, standard deviations, and results of the Tukey tests are reported in Table 5-4. Scores were also converted to T-scores $(\underline{\mathrm{M}}=50, \underline{\mathrm{SD}}=10)$ and graphed in Figure 5-3 and 5-4. Patterns in these results are discussed below.

The full Temptation scale continuously decreases with progression across the Stages of Change, with the largest decreases occurring between Preparation and Action and between Action and Maintenance. This result suggests that the level of Temptation is highly affected by length of time one has maintained a moderate drinking pattern. This pattern matches what 
was found for individuals progressing through the Stages of Change for smoking cessation (DiClemente et al., 1985).

In general the patterns for the subscales parallel the overall Temptation scale with a few notable exceptions. The two sub-scales measuring negative emotional states, Negative Affect and Social Anxiety both increase rather than decrease between Contemplation and Preparation. Although these are not significant differences they do suggest that those in Preparation might be more aware of being tempted to drink by negative emotional states than those in Contemplation. The other scale that diverges from the overall pattern is that Peer Pressure does not appreciable decrease between Preparation and Action as the other scale. This result suggest that the pressure from others to drink is not strongly affected by the behavior change, at least initially, or even that college students' susceptibility to peer pressure is increased shortly after reducing their drinking. This result might explain the high levels of relapse often reported in college populations. These patterns would have to be replicated before firm conclusions are drawn. 


\section{DISCUSSION}

The present study supports the use of the Temptations for College Drinking instrument to measure and investigate how students experience urges to drink immoderately. This instrument is composed of four correlated subscales organized in a hierarchical structure with one higher order Temptation factor. All four subscales and the general Temptation scale have excellent psychometric properties with good confimmatory model fit indicators, high item loadings, and excellent internal reliabilities. The results provide strong evidence that these scales are meaningfully related to immoderate drinking and its consequences. The findings also are consistent with predictions based on the Transtheoretical Model of Change and provide support for the application of this model to the behavior of immoderate drinking in a college population.

The four subscales instrument provides interesting information about college drinking. The Temptation instruments developed across many behaviors have shown consistent patterns of results, but the content and number of subscales of these instruments have been adapted to each behavioral domain. The four subscales found in this study, Peer Pressure, Negative Affect, Positive/Social, and Social Anxiety represent a unique set of subscales. Negative Affect and Positive/Social factors have been consistently found in studies of smokers. These studies also found a subscale labeled Habit Strength, which was investigated in the preliminary study, but did not produce an independent subscale, suggesting that drinking for college students is not substantially mediated by physiological processes.

Three of the scales, Positive/Social, Peer Pressure and Social Anxiety suggest the extent to which this behavior is mediated interpersonally. Peer Pressure has not been measured for adult smokers, but a similar subscale, labeled Partner Pressure, has been found in the clearly interpersonal behavior of safe sex practices (Redding, 1993). The importance of peer pressure in the social behavior of adolescents and young adults is widely acknowledged (see Jessor, 1987). 
The fourth scale, Social Anxiety measures an interpersonal cue which has not been previously articulated in the Transtheoretical Model based research into the Temptation construct, but has often been referred to in the literature on alcohol use (Foxcroft \& Lowe, 1993; Golding, Burnam, Benjamin, \& Wells, 1991). The extraction of this scale was surprising, as it was not found in the preliminary study and this type of situation was not hypothesized to separate from other negative affectual situations. Further work including the generation of new items to test the breadth of this construct and replicating a four subscale instrument would more firmly establish this dimension of the Temptation measure.

These subscales are strongly correlated with each other. Structural Modeling techniques show that these correlations can be orgamized as factors loading on a higher order general Temptation factor. This allows for both the generation of an overall Temptation score and separate subscale scores. These results integrate two separate models of behavior. The higher order factor suggests an underlying mechanism related to temptation to drink immoderately consistent with the self-efficacy model proposed by Bandura $(1977,1982)$. The separation of the four subscales also suggests that there is another phenomenon occurring in which students differentially respond to different situations, supporting research suggesting that individuals have meaningful differences in how they use alcohol and their response to internal and external cues (e.g. Cannon et al., 1990; Marlatt \& Gordon, 1985).

External validity evidence was very strong for these scales, demonstrating both their strong relation to outcome variables of alcohol consumption and alcohol-related negative consequences and to the predicted relationship with Stage of Change, the organizing construct of the Transtheoretical Model of Change.

The relationship between the Temptation scales and the Pros scale of the Decisional Balance scale proved to be very strong. One conceptualization of this relationship is that the Pros represent the reasons that the subjects are tempted. For example, the Pros tap reasons for drinking such as "feeling happier", "being more relaxed and less tense", and having "fun with friends" which seem likely to be reasons that students are tempted by negative affects, 
social anxiety, and in positive social situations, respectively. Further investigation is needed to determine the extent to which these two scales have unique predictive and explanatory utility.

The observed correlations with the Cons scales are also of interest. The lack of a significant relationship between a measure of the salience of the risks incurred by drinking and the temptation to drink suggests that interventions which focus on educating students as to the risks of drinking is unlikely to have a large affect on their temptation levels and their consumption patterns. The positive relationship between the salience of the negative emotional effects of drinking and being tempted to drink, especially when experiencing negative affects, suggests a positive feedback process that has been described as the basis for addictions. Namely, it is hypothesized that for some, their drinking makes them feel bad, which makes them want to drink. Alternatively, this relationship might reflect a general level of self-awareness where awareness of one's felt temptation to drink would be accompanied with awareness of its emotional effects.

These results have clear clinical implications. Clinicians should be aware that students' temptation to drink is elicited by social pleasures, pressures, and anxieties as well as internal negative emotional states. Students differentiate among these types of tempting situations and it would likely be useful if clinicians also did so and if they had effective ways of measuring these dimensions of temptation. Additionally, group interventions should be designed to intervene on all four types of temptations. Given the strong relationship to consumption patterns and alcohol-related experiences, the moderating of levels of temptations with a college population is likely to translate into reduced incidence of immoderate drinking and its consequences.

These results also suggest that temptation to drink is especially important in the prevention of relapse as evidenced by the Iarge late stage differences in levels of temptation. Most program interventions in college fail because of student relapse, and focusing on helping 
students continue to decrease their level of temptation even after months of successful behavior change might be an important intervention strategy.

Individualized, stage based interventions based on the Transtheoretical Model have been rigorously tested and proven to be more effective that other methods of promoting smoking cessation in adults (Prochaska, DiClemente, Velicer, \& Rossi, 1993; Velicer, Prochaska, Bellis, Rossi, \& Fava, 1993). A similar intervention for college drinking can be envisioned in which students would be assessed on a number of Transtheoretical variables including Stage of Change and Temptations. Students would then be given the individual feedback deemed most useful to them. The present study provides an instrument to assess the Temptations in such a system.

Finally, the limitations of this research should be noted along with recommendations for future research. First, the sample used was not representative although it is hoped that because of its large size the results would approximate population values for this university. Additionally, the sample was taken from one college, and drinking habits vary greatly across colleges (Wechsler et al., 1994). These results are likely to be applicable at least to other state universities, where drinking is allowed on campus and heavy drinking is common. These attributes describe many colleges and argue for the relevance of these findings. Secondly, this research is cross-sectional, and Iongitudinal study would determine if the patterns seen across Stages of Change would replicate longitudinally, although this has been the case with investigations of the Temptations for smokers (Prochaska, Velicer, Guadagnoli, Rossi, \& DiClemente, 1991). Longitudinal research would also allow for a greater understanding of the predictive utility of these scales. Lastly, intervention research would help determine how responsive or intractable the temptations to drink are to change attempts and how changing students' temptations to drink affects drinking habits and their consequences. 


\section{REFERENCES}

Allen, S. J. \& Hubbard, R. (1986). Regression equations for the latent roots of random data correlation matrices with unities on the diagonal. Multivariate Behavioral Research, $\underline{21}, 393-398$.

Bandura, A. (1977). Self-efficacy: Toward a unifying theory of behavioral change. Psychological Review, 84, 191-215.

Bandura, A. (1982). Self-efficacy: Mechanism in human agency. American Psychologist, 37, 122-147.

Bentler, P. M. (1989). EQS:Structural equations program manual. Los Angeles, California: BMDP Statistical Software.

Cannon, D. S., Leeka, J. K., Patterson, E. T., \& Baker, T. B. (1990). Principal component analysis of the inventory of drinking situations: Empirical categories of drinking by alcoholics. Addictive Behaviors, 15, 265-269.

Cattell, R. B. (1966). The Scree test for the number of factors. Multivariate Behavioral Research, 1, 245-276.

Clark, M. M., Abrams, D. B., Niaura, R. S., Eaton, C. A., \& Rossi, J. S. (1991). Selfefficacy in weight management. Journal of Consulting and Clinical Psychology, 59, 739-744.

DiClemente, C. C. (1986). Self-efficacy and the addictive behaviors. Journal of Social and Clinical Psychology, 4, 302-315.

DiClemente, C. C., Prochaska, J. O., \& Gilbertini, M. (1985). Self-efficacy and the stages of self change of smoking. Cognitive Therapy and Research, $\underline{9}, 181-200$.

Foxcroft, D. R. \& Lowe, G. (1993). Self-attributions for alcohol use in older teenagers. Addiction Research, 1 , 1-19. 
Golding, J, M., Burnam, M. A., Benjamin, B., \& Wells, K. B. (1991, August). Reasons for drinking and risk for alcohol abuse or dependence. Poster presented at the annual meeting of the American Psychological Association, San Francisco, CA.

Horn, J. L. (1965). A rationale and test for the number of factors in factor analysis.

Psychometrika, $\underline{30}, 179-185$.

Jackson, D. N. (1970). A sequential system for personality scale development. In Spielberger (Ed.), Current topics in clinical and community psychology (Vol. 2, pp. 61-96). New York: Academic Press.

Jessor, R. (1987). Problem-behavior theory, psychosocial development, and adolescent problem drinking. British Journal of the Addictions, 2 2, 331-342.

Marcus, B. H., Selby, V. C., Niaura, R. S., \& Rossi, J. S. (1992). Self-efficacy and the stages of exercise behavior change. Research Quarterly for exercise and sport, $\underline{63}, 60-$ 66.

Marlatt, G. A. \& Gordon, J. R. (1980). Determinants of relapse: Implication for the maintenance of behavior change. In P. Davidson \& S. Davidson (Eds.), Behavioral medicine: Changing health lifestyles (pp. 410-452). New York: Brunner/Mazel.

Marlatt, G. A. \& Gordon, J. R. (1985). Relapse prevention: Maintenance strategies in the treatment of addictive behaviors. New York: Guilford Press.

Perry, C. L., Baranowski, T., \& Parcel, G. S. (1990). How individuals, environments, and health behavior interact: Social learning theory. In K. Glanz, F. M. Lewis, \& B. K. Rimer (Eds.), Health behavior and health education: Theory, research, and practice (pp. 161-186). San Francisco: Jossey-Bass Inc.

Prochaska, J. O., DiClemente, C. C., Velicer, W. F., Ginpil, S., \& Norcross, J. C. (1985). Predicting change in smoking status for self-changers. Addictive Behaviors, $\underline{10}, 395-$ 406. 
Prochaska, J. O., DiClemente, C. C., Velicer, W. F., \& Rossi, J. S. (1993). Standardized, individualized, interactive and personalized self-help programs for stages of smoking cessation. Health Psychology, 12, 399-405.

Prochaska, J. O., Velicer, W. F., Guadagnoli, E., Rossi, J. S., \& DiClemente, C. C. (1991). Patterns of change: Dynamic typology applied to smoking cessation. Multivariate Behavioral Research, 26, 83-107.

Prochaska, J. O., Harlow, L. L., Redding, C. A., Snow, M. G., Rossi, J. S., \& Velicer, W. F. (1990). Stages of Change, Self Efficacy, and Decisional Balance of condom use in a high HIV risk sample. Technical report to the Centers for Disease Control, contract grant $\# 0-4115-002$.

Redding, C. A. (1993). The transtheoretical model applied to safer sex behavior among university students: A cross-sectional investigation. Unpublished doctoral dissertation, University of Rhode Island, Kingston, RI.

Snow, M. G. (199I). A transtheoretical analysis of strategies in the recovery process from alcohol problems. Unpublished doctoral dissertation, University of Rhode Island, Kingston, R.I.

Velicer, W. F. (1976). Determining the number of components from the matrix of partial correlations. Psychometrika, $\underline{41}, 321-327$.

Velicer, W. F., DiClemente, C. C., Rossi, J. S., \& Prochaska, J. O. (1990). Relapse situations and self-efficacy: An integrative model. Addictive Behaviors, 15, 271-283.

Velicer, W. F., Prochaska, J. O., Bellis, J. M., Rossi, J. S., Fava, J. L., \& Steiger, J. H. (1993). An expert system intervention for smoking cessation. Journal of the Applied

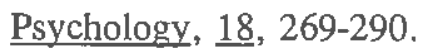

Wechsler, H., Davenport, A., Dowdall, G., Moeykens, B., \& Castillo, S. (1994). Health and behavioral consequences of binge drinking in college. Journal of the American Medical Association, 272, 1672-1677. 
Zwick, W. R. \& Velicer, W. F. (1986). Comparison of five rules for determining the number of components to retain. Psychological Bulletin, 99, 432-442. 
Table 5-1: Principal component loadings for exploratory analysis of Temptation items

$\begin{array}{ll}\text { Scale 1: Peer Pressure } & \text { I } \\ \text { 1 - When other people encourage me to have a drink, } & .82 \\ \text { 2 - When my friends push me to keep up with their drinking. } & .81 \\ \text { 3 - If I go to a party where there is a lot of drinking. } & .76 \\ \text { 4 - When I feel like keeping up with my friends' drinking. } & .76 \\ \text { 5 - When I am with others who are focusing on drinking. } & .74 \\ \text { 6 - When I am with others who are drinking a lot. } & .72 \\ \text { 7 - When there are drinking games going on. } & .69 \\ \text { 8 - When I am offered a drink by someone. } & .56\end{array}$

\section{Scale 2: Negative Affect}

1 - When things are not going my way and I am frustrated, $\quad .89$

2 - When I am feeling depressed. $\quad .85$

3 - When I am very anxious and stressed. $\quad .83$

4 - When I have my feelings hurt. $\quad .82$

5 - When I am feeling angry. $\quad .80$

\section{Scale 3: Positive/Social}

1 - When things are going really well for me. $\quad .84$

2 - When I am really happy. $\quad .84$

3 - When I am excited. $\quad .69$

4 - When I am having fun with friends.

\section{Scale 4: Social Anxiety}

1 - When I am nervous about being socially outgoing. $\quad .78$

2 - When I am with someone I am attracted to. $\quad .72$

3 - When I am feeling shy $\quad .70$

4 - When I am anxious about sex. 
Table 5-2: Means, standard deviations, coefficient alphas and scale intercorrelations for the Temptation full scale and subscales for both samples

Sample $1(\mathrm{~N}=302)$

\begin{tabular}{|c|c|c|c|c|c|c|c|}
\hline \multirow[b]{2}{*}{ SCALE } & \multirow[b]{2}{*}{ Mean } & \multirow[b]{2}{*}{ SD } & \multirow[b]{2}{*}{ Alpha } & \multicolumn{4}{|c|}{ Correlations } \\
\hline & & & & 1 & 2 & 3 & 4 \\
\hline 1 - Temptation Scale & 2.71 & .89 & .95 & & & & \\
\hline 2 - Peer Pressure & 2.94 & 1.01 & .92 & .89 & & & \\
\hline 3 - Negative Affect & 2.41 & 1.10 & .92 & .77 & .51 & & \\
\hline 4 - Positive/Social & 2.90 & 1.10 & .88 & .79 & .65 & .44 & \\
\hline 5 - Social Anxiety & 2.46 & 1.10 & .86 & .84 & .63 & .62 & .60 \\
\hline
\end{tabular}

Sample 2 (N=305)

\begin{tabular}{cccccccc}
\hline & & & & \multicolumn{4}{c}{ Correlations } \\
\cline { 6 - 8 } SCALE & Mean & SD & Alpha & 1 & 2 & 3 & 4 \\
\hline 1 - Temptation Scale & 2.76 & .85 & .95 & & & \\
2 - Peer Pressure & 2.99 & .98 & .93 & .90 & & \\
3 - Negative Affect & 2.49 & 1.09 & .92 & .74 & .48 & \\
4 - Positive/Social & 2.91 & 1.01 & .88 & .80 & .70 & .40 \\
$\mathbf{5}$ - Social Anxiety & 2.49 & 1.03 & .85 & .84 & .67 & .59 & .61 \\
\hline
\end{tabular}

* All correlations are significant at $\mathrm{p}<.001$ 
Table 5-3: Correlation of Temptation full scale and subscales with external validity variables

\begin{tabular}{lccccc}
\hline & Temptation & $\begin{array}{c}\text { Peer } \\
\text { Pressure }\end{array}$ & $\begin{array}{c}\text { Negative } \\
\text { Affect }\end{array}$ & $\begin{array}{c}\text { Positive/ } \\
\text { Social }\end{array}$ & $\begin{array}{c}\text { Social } \\
\text { Anxiety }\end{array}$ \\
\hline Age & -.05 & -.04 & -.10 & .00 & -.01 \\
\hline
\end{tabular}

Alcohol Consumption

$\begin{array}{llllll}\text { DRINKS } & .49 & .47 & .29 & .47 & .38 \\ \text { DAYS } & .51 & .48 & .31 & .48 & .42 \\ \text { INTOX } & .24 & .23 & .12 & .26 & .15\end{array}$

Alcohol Related Experiences

$\begin{array}{lrrrrr}\text { EXCESS } & .59 & .56 & .36 & .53 & .47 \\ \text { DISTRESS } & .52 & .43 & .45 & .36 & .47 \\ \text { PROBLEM } & .33 & .30 & .27 & .20 & .28\end{array}$

Decisional Balance

\begin{tabular}{lccccc}
\hline PROS & .75 & .60 & .56 & .60 & .73 \\
CONS-P & .02 & -.02 & .06 & -.06 & -.06 \\
CONS-A & .16 & .11 & .24 & .02 & .17 \\
\hline
\end{tabular}

Note: All correlations greater than or equal to .10 are significant at $\mathrm{Q}<.05$ 
Table 5-4: Means and standard deviations for Temptation scales by the Stages of Cessation

\begin{tabular}{|c|c|c|c|c|c|c|}
\hline & & $\mathbf{P C}$ & CONT & PREP & ACTION & MAIN \\
\hline \multicolumn{7}{|l|}{ I. Second-order Scale } \\
\hline \multirow[t]{2}{*}{ Temptation Scale } & Mean & 3.19 & 3.07 & 3.01 & 2.74 & 2.35 \\
\hline & SD & .65 & .62 & .68 & .71 & .77 \\
\hline \multicolumn{7}{|l|}{ II. First-order Subscale } \\
\hline \multirow[t]{2}{*}{ Peer Pressure } & Mean & 3.42 & 3.37 & 3.13 & 3.12 & 2.52 \\
\hline & SD & .79 & .74 & .69 & .84 & .90 \\
\hline \multirow[t]{2}{*}{ Negative Affect } & Mean & 2.85 & 2.59 & 2.80 & 2.48 & 2.19 \\
\hline & SD & 1.04 & .87 & 1.06 & 1.05 & 1.00 \\
\hline \multirow[t]{2}{*}{ Positive/Social } & Mean & 3.42 & 3.30 & 3.16 & 2.73 & 2.54 \\
\hline & $S D$ & .82 & .70 & .95 & .93 & 1.05 \\
\hline \multirow[t]{2}{*}{ Social Anxiety } & Mean & 2.92 & 2.82 & 2.88 & 2.34 & 2.01 \\
\hline & SD & .96 & .92 & .94 & .92 & 1.02 \\
\hline
\end{tabular}


Figure 5-1: Correlated structural model for Temptations scales

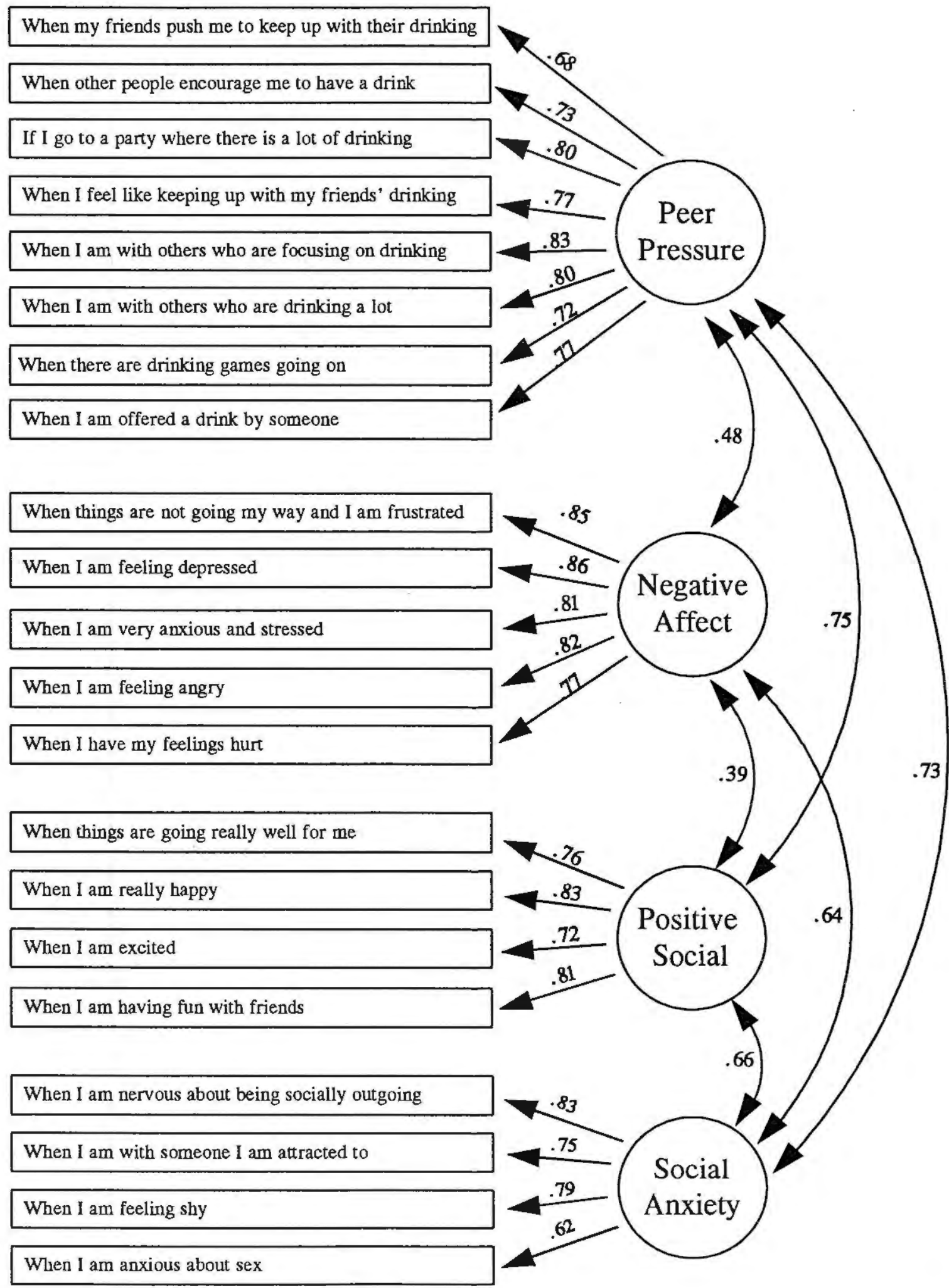


Figure 5-2: Hierarchical structural model for Temptations scales

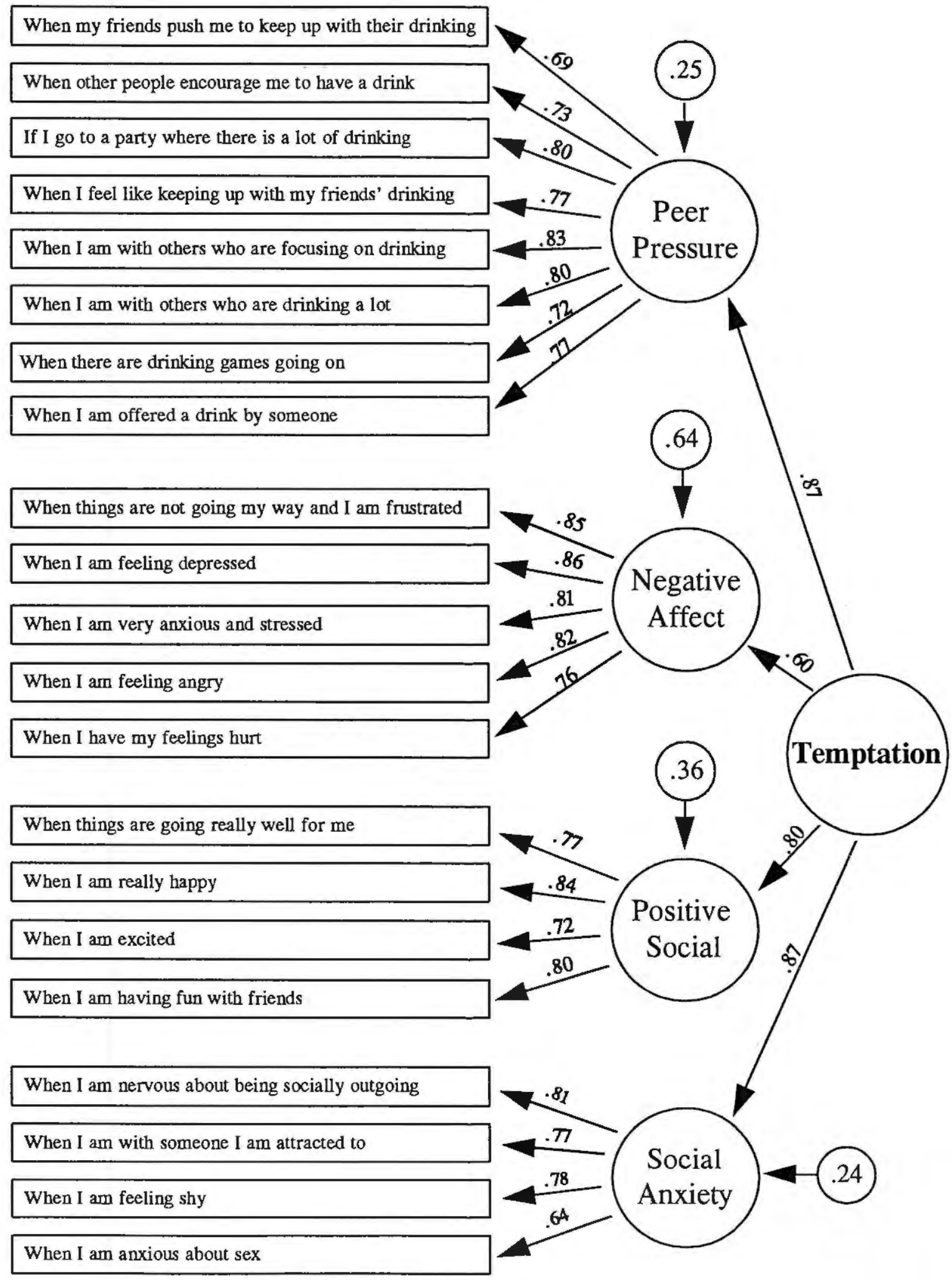


Figure 5-3: Full Temptation scale by Stage of Cessation

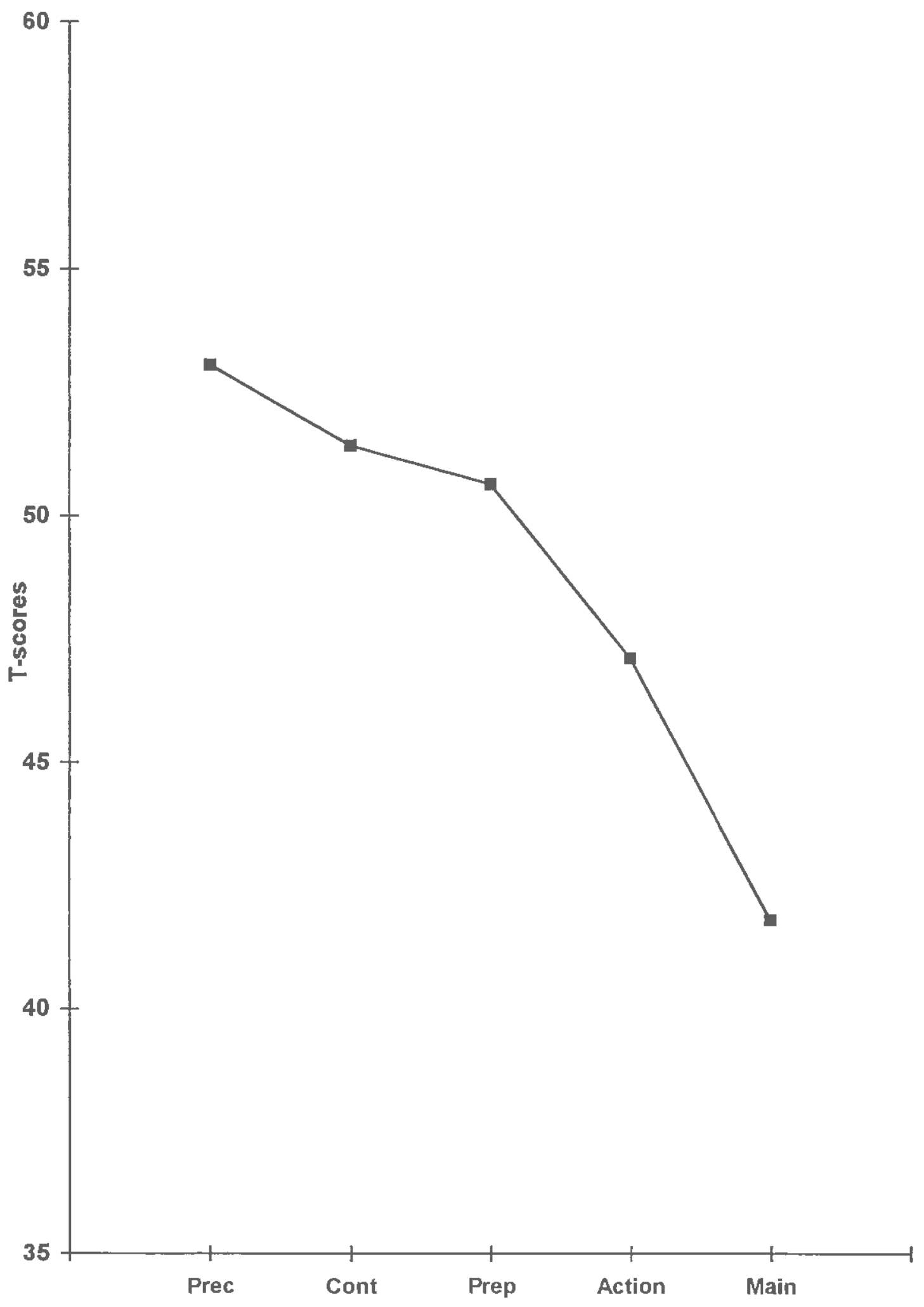


Figure 5-4: Temptation subscales by Stage of Cessation

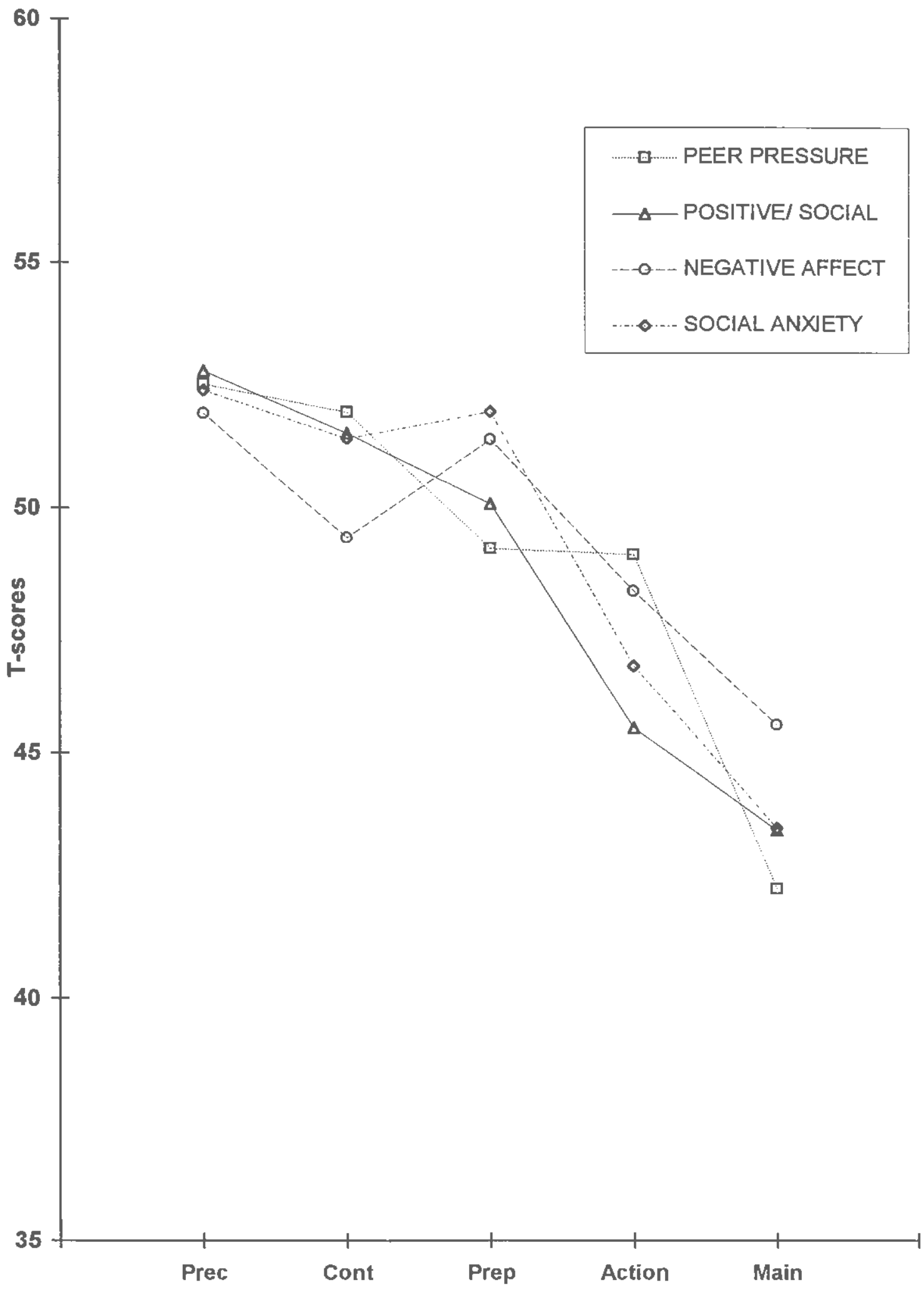




\section{PART VI. STUDY 5: PROCESSES OF CHANGE AND RESISTANCE FOR \\ IMMODERATE ALCOHOL USE}

\section{INTRODUCTION}

Immoderate drinking on college campuses is endemic (Kraft, 1988; Wechsler, Davenport, Dowdall, Moeykens, \& Castillo, 1994) and has been a recognized health concern for decades. Despite years of efforts, structured interventions have generally not had meaningful long-term results. It is also known that students often moderate their drinking habits on their own, either during college or shortly thereafter (Harford, 1984). In a survey at the same university as the present study was conducted, Stevenson, Mitchell and Migneault (1990) found that freshmen drank on the average of 5.2 drinks per occasion, whereas seniors drank only 3.4 drinks per occasion. These figures suggest that there is a naturally occurring change process resulting in significant moderation of college students' drinking over time.

The Transtheoretical Model of Change is a model that has paid special attention to natural change processes for a variety of behaviors, yet this model has not been substantially applied to college drinking behaviors. This model has its origins in a theoretical integration of the processes that are mobilized by the major psychotherapeutic modalities. These processes, called the Processes of Change, describe a set of overt and covert activities that are used to intentionally change a behavior. There were originally thirteen hypothesized processes (Prochaska, 1979). Initial research on self changing smokers found ten separate and measurable processes (Prochaska \& DiClemente, 1983). These ten were later found to possess a hierarchical structure, separating into five experiential and five behavioral processes (Prochaska, Velicer, DiClemente, \& Fava, 1988). The five experiential processes are:

Consciousness Raising, Dramatic Relief, Environmental Re-evaluation, Social Liberation, and Self Reevaluation. The five behavioral processes are: Counter Conditioning, Stimulus 
Control, Reinforcement Management, Helping Relationship, and Self Liberation. Although this model has developed to include other constructs, the Processes of Change remain central.

Research on the Processes of Change across a wide range of behaviors has lent strong support to the validity and usefulness of this construct. Similar sets of processes have been found in exercise, cocaine use, therapy changers, weight change, and safe sex behavior (Marcus, Rossi, Selby, Niaura, \& Abrams, 1992; Prochaska \& DiClemente, 1985; Redding, 1993; Rossi \& Rossi, 1993; Rosenbloom, 1991; Rossi, 1992).

Another construct of the Transtheoretical model is the Stages of Change. This construct provides an organizing structure to investigate the other constructs of the model, including processes. There are five primary stages: Precontemplation (not considering changing the target behavior), Contemplation (intending to change in the next six months), Preparation (planning change in the next 30 days), Action (having changed in the last six months), and Maintenance (having changed the behavior more than six months ago). These stages have been validated in many different behavioral domains, and where longitudinal research has been done, have been highly predictive of future behavior change (Prochaska, Velicer, Guadagnoli, Rossi, \& DiClemente, 1991).

The Processes of Change have been found to possess an orderly relationship to the Stages of Change. In a longitudinal study of smokers it was found that the experiential processes were used more heavily in the initial stages and the behavioral processes were used more in the later stages (Prochaska et al., 1991). More specifically it has been found that Precontemplators use the processes the least, that Contemplators use Consciousness Raising and Dramatic Relief the most, Self Reevaluation is used as one moves from Contemplation to Preparation, and Self Liberation is a process used as individuals move from Preparation to Action. The behavioral processes are used more in Action and Maintenance. Furthermore it has been shown that not only are processes maximized at appropriate stages by successfuI changers but that those who use processes that are not stage appropriate are more likely to relapse. Those individuals in Action and Maintenance who use high levels of Self 
Reevaluation are more at risk of relapse. This stage-process topology allows for the construction of individualized treatment interventions that have been proven to be superior to non-staged matched interventions (Prochaska, DiClemente, Velicer, \& Rossi, 1993).

Although the initial research on the Processes of Change was conducted with abstinence based criteria (e.g. smoking) there is ample evidence to suggest that the processes will be useful in examining the effort to limit alcohol intake. For example, in the area of dietary fat intake where the criteria is also control of consumption, the processes have been validated (Rossi \& Rossi, 1993). Other results show that light smokers use many of the processes of change to a greater degree than heavy smokers (Rossi, Prochaska, \& DiClemente, 1988).

The only published research to date measuring the Processes of Change for alcohol use is the research on sober alcoholics (Prochaska, Rossi, \& Snow, 1992). This abstinence based research found eight processes. Five of these were from the original ten hypothesized (Stimulus Control, Helping Relationship, Consciousness Raising, Dramatic Relief, Social Liberation), two were combinations of two processes (Contingency Management and Counter Conditioning, Self and Environmental Reevaluation), and the other was a process, called Substance Use, that was hypothesized to be the process of substituting other substances for alcohol. Although measured, Substance Use did not prove to be useful in this investigation. The only process not measured was Self Liberation. The finding of a smaller set of processes in this study might represent methodological issues such as moderate sample size or the restrictive sampling of stages. Alternatively it might represent a slightly different change process for alcoholics remaining abstinent than found for other behaviors.

Besides the research using the Transtheoretical Model there has only been limited investigation into the processes used by individuals successful in their attempts to control use of addictive substances. In the 1960's Zinberg and colleagues started to investigate the controversial phenomenon of controlled illicit substance abuse (Zinberg \& Fraser, 1979; Zinberg, Harding, \& Winkeller, 1977). They found that those individuals who maintained 
control over the use of these substances tended to use externally-based control processes, especially social ritual and social sanctions, which they often developed in small subgroups. These investigators felt that this analysis could be extended to legal substances such as alcohol, and pointed out the myriad of social sanctions and rituals surrounding alcohol that serve the purpose of promoting and yet restricting use. This hypothesis has not been investigated empirically.

More recently, Werch (1990) has investigated the strategies that undergraduates use to intentionally limit their consumption of alcohol. He found seven strategies that were used. Of these seven, four seem to be tapping the processes of Counter Conditioning, Reinforcement Management, and Stimulus Control. Others seem to be measuring specific tactics (e.g. "I eat before drinking") whose relationship to the Processes of Change of the Transtheoretical Model is unclear. This research was based on self-help manuals designed to help establish controlled drinking, and therefore was predisposed to find processes used predominately in the Action stage.

Werch found that these strategies significantly predicted the outcome variables of quantity, frequency, alcohol related problems, and drinking and driving. Interestingly, he also found that the level of overall use of strategies generally had a curvilinear relation to his outcome variables, with use of strategies generally going up between low and moderate outcome variable levels, and going back down with heavy use. This pattern contrasts with the more linear relationship that Rossi et al. (1988) found for cigarette smoking and suggests that moderate drinkers are using strategies to either maintain or change their drinking behaviors, whereas light and heavy drinkers expend less effort maintaining their alcohol consumption patterns.

The above research clearly suggests that the Processes of Change should be a useful construct with which to investigate problematic or immoderate vs. controlled or moderate alcohol use. Although there are ten processes that have been most clearly supported, two additional Processes of Change were investigated in this study. This allows for both the 
validation of the original model and for its continued development. The first of these is Interpersonal Systems Control. This process is theoretically related to Stimulus Control, as it is the control of interpersonal cues to the behavior in question. This process has been investigated for other behaviors with mixed results, at times being found as an independent scale and at other times merging with Stimulus Control. It was hypothesized that it would be relevant for such an interpersonal activity as college drinking.

The second additional Process of Change has not been previously investigated within the context of the Transtheoretical Model of Change. This process, labeled Self Monitoring, is based on the interventions designed by Miller (1987) to help excessive drinkers develop controlled drinking habits. This process entails the conscious effort to increase one's awareness of aspects of one's consumption of alcohol and the internal experiences that are associated with this consumption. It is hypothesized that this information is then used to more fully control drinking behavior. Definitions for all the processes investigated in this study are presented in Table 6-1.

This study also attempted a systematic investigation of a set of processes that college students use to resist changing immoderate drinking habits. These processes, labeled Processes of Resistance, are conceived as activities, largely intrapsychic in nature, which are used to resist pressures to change a behavior. In one published study constructs that were conceived of as ineffective defensive coping mechanisms were investigated but not systematically integrated into the model (Prochaska, Norcross, Fowler, Follick, \& Abrams, 1992). In the present study three Processes of Resistance were hypothesized to be meaningfully related to immoderate alcohol use. Two of these processes are taken from psychodynamic defense mechanism theory (see Vaillant, 1977) and are labeled Repression/Denial and Rationalization. The third process, Reactance, is taken from research on adolescent rebellion and has received some support as a mechanism leading to increased substance use in certain situations (Engs \& Hanson, 1989). 
This study assesses the applicability of the Processes of Change, a central construct of the Transtheoretical Model of Change to the behavioral domain of immoderate alcohol use in a college population. It will also systematically investigate an extension of the process construct to include the Processes of Resistance. These results will have important implications for the development of Transtheoretical Model based interventions for college drinking. Such interventions have been very successful in other behavioral domains, and hold much promise for college drinking. 


\section{METHOD}

\section{SUBJECTS}

Subjects were 629 students surveyed at the University of Rhode Island in the fall of 1993 and spring of 1994 between the ages of 18 and 25 years and 11 months. Most of the subjects were recruited from undergraduate classes in the psychology department, although a variety of other classes were also sampled including physical education, pharmacology, nutrition, and sociology. A smail percentage was recruited from the fraternities and the university health center.

The sample was $66.3 \%$ female, and $94 \%$ white. The distribution across the classes was $17.4 \%$ freshmen, $32.2 \%$ sophomores, $19.1 \%$ juniors, $26.2 \%$ seniors, and $5.3 \%$ either fifth-year or non-matriculated. The average age was 21.3 years. Of these students $47 \%$ were classified as in-state and $39 \%$ lived in dormitories, $10 \%$ lived in fraternities or sororities, and $51 \%$ lived off campus.

The use of alcohol in this sample was substantial. Using a criterion of at least one drinking occasion in the last 30 days, $92 \%$ were classified as active drinkers. These subjects drank on average 8.6 days a month and consumed on average 5.3 drinks per occasion. There were substantial differences in drinking by gender with drinking women averaging 7.3 days per month and 4.4 drinks per occasion. For men these figures were 11.0 days and 6.9 drinks. Drinking women reported an average maximum amount drunk on any occasion in the past month as 6.8 , and men as 12.1 .

\section{INSTRUMENTS}

The survey administered contained 282 questions, including, of specific interest to this study, the following item sets. The full survey instrument is presented in Appendix A. 


\section{Demographics}

A set of 22 items asked about basic demographic information and drinking history. Variables examined in this study include age, gender, college class, living situation, number of days in the last month that alcohol was consumed (Days), the number of drinks consumed during a typical drinking occasion (Drinks), and the number of drinks consumed before subjects start to feel intoxicated (Intoxication).

\section{Processes of Change and Resistance Item Set}

A set of 96 items hypothesized to measure the 12 Processes of Change and 3 Processes of Resistance was included in the survey. Eleven of the Processes of Change had been previously conceptualized and investigated within the context of the Transtheoretical Model. One Process of Change, Self Monitoring, and the three Processes of Resistance had not. Definitions for these processes were taken from other literature. Between eight and twelve items were generated for each process by adapting items from other behavioral domains such as smoking (Prochaska et al., 1988), or from surveys on alcohol use with other populations (Snow, 1991), or by creating items to measure aspects of the proposed processes that were deemed especially relevant to a college population. Definitions of each of the processes are presented in Table 6-1.

Three expert judges assigned each item to one of the thirteen hypothesized processes. Items that were judged by at least two judges to measure the hypothesized stage were reviewed. Of these, 96 were chosen to measure the thirteen processes (5-8 items per process). Subjects were asked to rate the frequency with which they engaged in or experienced each item in the last month using a 5-point Likert scale with $I=N e v e r$ and $5=$ Repeatedly. 


\section{Stages of Change}

Algorithmic method. A staging algorithm used answers from a branched set of five items to classify subjects into one of nine groups that represented the five Stages of Acquisition and the five Stages of Cessation with one group representing both the Maintenance stage of acquisition and the Precontemplation stage of cessation. In the present study only the stages of cessation were investigated $(\mathrm{N}=426)$. The algorithm used was developed and investigated in Study I and used a criterion of usually drinking 4 or more drinks at least once in a typical week for women and 5 or more for men. The development and investigation of this staging method is presented in Study I.

Continuous measure. Three scales which compose the 21-item University of Rhode Island Change Assessment for Alcohol instrument (URICA-A) were used to measure stage related attitudes. These scales included a Precontemplation scale which measures acceptance of heavy drinking, a Contemplation scale which measures ambivalence about one's drinking habits and plans to change them, and a Maintenance scale which measures attitudes consistent with having reduced one's drinking and maintained them at more moderate levels. Scale alphas are .70 for the Precontemplation scale, .83 for the Contemplation scale, and .78 for the Maintenance scale.

\section{Decisional Balance Scales}

A set of 18 items that measure the Decisional Balance construct of the Transtheoretical Model of Change was included. These items compose three scales. The Pros scale measures the importance of 10 benefits of drinking in subjects' decisions about alcohol consumption. There were two Cons scales, Cons $=\mathrm{P}$ and Cons- $\mathrm{A}$. The Cons-P is a 5-item scale that measures the importance of the risks of concrete or practical consequences of drinking in their decisions. The Cons- $\mathrm{A}$ is a 3-item scale which assesses the importance of the emotional effects of drinking. These scales have internal consistencies as measured by Cronbach's Alpha of $.92, .84$ and .68 , respectively. 


\section{Temptation Scale}

A 21-item scale that measures temptations to drink excessively in response to a variety of situational cues was included in the survey. The overall scale is composed of four correlated subscales that are labeled Peer Pressure, Negative Affect, Positive/Social, and Social Anxiety, but an overall score measuring general temptation to drink excessively was used in this study. This scale's internal consistency as measured by Cronbach's Coefficient Alpha is 95. See Study IV for the development of this instrument.

\section{Alcohol-Related Experiences}

Three scales, developed in Study I, that measure types of negative alcohol related experiences were inciuded as a central validity variable. The scales are composed of 23 items developed from an original set of 31 items and are labeled Excess, Distress, and Problem. The three scales have moderate inter-scale correlations ranging from .37 and .44 and high internal consistencies as measured by Cronbach's Alpha ranging from .80 to .88 . The Excess scale is hypothesized to measure problems associated with short-term excessive or binge drinking. The Distress scale measures experiences of emotional or introspective distress, and the Problem scale measures consequences of alcohol consumption that are associated with long term problem drinking.

Subjects were asked to circle the number of times in the last 12 months that they had had each experience related to their alcohol consumption. The following 6-point response scale was provided: $0,1,2,3-5,6-9,10$ or more times.

\section{PROCEDURE}

The items used in this study were included within a larger survey that assessed additional aspects of drinking behaviors and attitudes. Informed consent was obtained and subjects were assured of the confidentiality of their responses (See Appendix B for a copy of the consent form). Surveys were completed during class time, or subjects took the survey 
home and returned it. Most, although not all, of those doing the survey on their own time received a small amount of class credit for completing the survey. A few students who completed the survey at the health services center were given a pen emblazoned with the university name in exchange for completing the survey.

\section{ANALYTIC PLAN}

The investigation of the Processes of Change and the Processes of Resistance was designed to assess the applicability of the Transtheoretical Model to the behavior of immoderate college alcohol use, to explore four new processes, and to develop a psychometrically sound scale instrument. Further analysis investigated the external validity of the processes.

The initial exploration of the Processes of Change item set used structural modeling techniques. These techniques allow for the development of new measurement scales within the structure of a predetermined model. The method is justifiable when a well developed and validated model is available. The Processes of Change represents a multicomponent construct that has a strong theoretical base (Prochaska, 1979; 1984; 1994) and has been empirically validated across numerous populations and behaviors (see Rossi, 1992). Structural modeling techniques are aIso especially useful in assessing multi-dimensional constructs which have high inter-component correlations and/or hierarchical structures, as is the case with the Processes of Change.

Optimally, the results of using structural modeling in an exploratory manner are confirmed on an independent sample. Model modifications based on structural model statistics reflect both population characteristics and chance sample attributes and replication is an effective method of identifying model modifications based on unstable sample characteristics. The large size of the measurement model for the Processes of Change, results in a large number of parameters to be estimated and demands a large sample to produce stable results. The sample in this study is insufficiently large to split into two sub-samples of 
adequate size, so confirmatory analyses were not attempted. In the absence of replication, interpretation of the results of this study will have to be tentative and will await further research efforts for confirmation.

After the development of the measurement model, hierarchical models based on previous research were tested on the Processes of Change.

The Processes of Resistance have had minimal previous theoretical or empirical development. The smaller set of 22 original items allowed for the sample to be split. Both exploratory principle components analysis and confirmatory structural modeling were conducted. The exploration of the Processes of Change and Resistance was completed with the testing of hierarchical models which combine both types of processes to assess their interrelations.

After instrument development was completed, external validity was investigated. The empirical relation of the Processes of Change and Resistance to both outcome variables such as alcohol consumption variables and negative experiences related to drinking and to Transtheoretical Model variables such as the Stage of Change, Decisional Balance, and Temptations were assessed. 


\section{RESULTS}

\section{The Processes of Change}

\section{Initial exploratory structural modeling}

Subjects were eliminated from this analysis for two reasons. First, cases that had missing data on more than $10 \%$ of the process of change items were eliminated $(5.1 \%$ of the sample). Second, cases which had a mean response across all 74 items of 1.5 or less were eliminated (1.7\% of the remaining sample). This last criterion was chosen to eliminate subjects that had very low endorsement of items. Cases with such an extremity response bias are not useful in the exploration of the dimensionality of an item set and can inflate intercomponent correlations (Jackson, 1970; Velicer, DiClemente, Rossi, \& Prochaska, 1990).

One item hypothesized to measure Stimulus Control was deleted because it had a very low mean $(\overline{\mathrm{x}}=1.38)$ and high kurtosis $(\mathrm{k}=6.8)$. Responses on the remaining 73 items were analyzed using the EQS structural modeling program (Bentler, 1989). Three models were run. The first was the Null Model, which posits 73 independent variables, and was not expected to fit the data but generated a set of statistics to which the other models can be compared. The second was the Uncorrelated Model and consisted of 12 uncorrelated processes and the third, the Correlated Model, of 12 correlated processes. Theory and past empirical research suggested that the Uncorrelated Model would not fit the data as well as the Correlated Model, but it was calculated as another baseline of comparison.

A set of fit indices including Chi square statistic, Comparative Fit Index (CFI), NonNormed Fit Index (NNFI), Root Mean Square (RMS), and Incremental Fit Index, type 2 (IFI2) were calculated to assess model fit. Since these indices produced a similar pattern of results, only the IFI2 and RMS are presented here. These fit indices indicated that the correlated 12 factor model was superior to the Uncorrelated Model (Correlated Model: $\mathrm{IF1}=.725, \mathrm{RMS}=.0598 ;$ Uncorrelated Model: $1 \mathrm{FI} 2=.491, \mathrm{RMS}=.1658)$. 
The poor fit of even the Correlated Model is expected at this early stage of instrument development because it is presumed that there is a sizeable subset of items that are inefficient measures of the constructs they are hypothesized to measure. The Correlated Model was used to refine this item set. To remove items or otherwise modify the model, a number of indicators were used including item loadings, residuals, and the LM statistic (Bentler, 1989). The LM statistic indicates the amount of model improvement that would occur if a nonestimated path were allowed to be non-zero. This can be used as an indicator of item complexity. An iterative process was used, in which a small number of the most clearly indicated model changes were made, the resultant model was refit to the data, and then further modifications were made. This iterative process was used because model modifications can affect any of the estimated model parameters.

Item loadings were examined first, and those with the lowest loadings were eliminated. In the final stages of model modification all items with loading below .50 were eliminated, One factor, Social Liberation, was eliminated in this iterative process. Also one item that was hypothesized to load on Environmental Reevaluation was found to have a much higher loading on Self Reevaluation. This item ("I stop and think that my drinking is causing problems for others"), although fitting the original definition of Environmental Reevaluation, uses the word 'my' in it, making it an appropriate measure of Self Reevaluation. It was allowed to load solely on this factor.

It was found that Stimulus Control was reduced to three items and that one of these items reduced the Cronbach's Alpha of the scale. Furthermore, initial runs of hierarchical Models suggested that the correlation between this process and Interpersonal Systems Control was not accounted for by the higher order factors. Because of these empirical findings, and because Interpersonal Systems Control can be thought of as a specific type of Stimulus Control (i.e. controlling interpersonal cues), the items for these two scales were combined and loaded on one process which retained the label Stimulus Control and appropriate model 
modifications were completed. This resulted in a long scale of eight good items, of which two items were deleted using scale breadth considerations resulting in a six-item scale.

This analysis resulted in the elimination of 27 items, with the 46 items remaining loading on ten factors representing Processes of Change. All factors had at least one item removed, and three factors were reduced to three items, the minimum for good construct identification. The other factors were left with four, five, or six items. The model is presented in Figure 6-1 with the interfactor correlations in Table 6-2.

The fit on this model was IFI2 $=.860$ and $\mathrm{RMS}=.042$. Conventions in the field are IFI $\geq .90$ and RMS $\leq .06$ as indicators of excellent model fit. The somewhat lower than optimal value for the IFI2 is probably caused by a high number of items with moderate complexity across the factors. These secondary loadings are un-estimated (constrained to zero) and therefore reduce model fit. It is also the case that a model as large as this one and with a number of scales of moderate length ( 5 or 6 items) also penalizes model fit by creating many non-estimated paths. As a further test of the model, item parcels were randomly created on scales that had more than three items to create three manifest indicators for each process. The fit indices for this model were $\mathrm{IFI} 2=.922$ and $\mathrm{RMS}=.037$, which represent excellent model fit.

As a further check of scale cohesion, Cronbach's Coefficient Alphas were calculated for each scale, and the change in alpha with each item deleted singly was calculated. It was found that all items contribute to their scale's internal consistency. Alphas ranged from .66 to .86 with a mean of .76 . Scale scores were calculated by taking an unweighted mean of item responses for subjects who had answered at least $50 \%$ of the respective scale's items. Scale means, standard deviations and internal consistencies are presented in Table 6-3. Pearson interscale correlations were also calculated and are presented in Table 6-4.

As can be seen there are a number of correlations that are quite high. Especially high are the correlations found among the processes Reinforcement Management, CounterConditioning, and Self-Liberation and among Consciousness Raising, Environmental 
Reevaluation, and Dramatic Relief. These probably indicate that at least in this sample the dimensionality of this instrument is less than 10 . Because it is likely that this is at least partially due to sample characteristics, such as the low numbers of subjects in the middle Stages of Change, the separate scales were preserved to more fully assess the applicability of the Transtheoretical Model of Change as previously developed to this population.

\section{$\underline{\text { Process of Change Hierarchical Models }}$}

In previous research hierarchical structural models have been found to parsimoniously account for interscale correlations (Prochaska et al., 1988). Two hierarchical models were initially fit to the process of change data and then model modifications were explored. The first was the One Factor Hierarchical Model, suggesting that all the inter-factor correlations could be more parsimoniously explained by a single second order factor representing a general tendency for subjects to engage in Processes of Change. The second, the Two Factor Hierarchical Model, was based on the finding that in other behavioral domains two secondorder correlated factors, labeled Experiential and Behavioral, best explained the data. This model hypothesizes that subjects differentiate processes that are more experiential from those which entail more overt behavior change.

One process, Self Monitoring, a process not previously investigated within the Transtheoretical Model, was hypothesized to load more highly on the Behavioral factor, but was originally allowed to load on both factors as a test of this hypothesis. The other nine processes were initially assigned to either the Experiential or Behavioral factor based on previous research (Prochaska et al., 1988). Modifications in these models were then explored.

The investigation of the Two Factor Hierarchical Model showed that Self Monitoring loaded higher on the Behavioral factor as hypothesized. The results also showed a low loading for Helping Relationship and Self Reevaluation on the Experiential factor. In subsequent model runs these were allowed to load on both factors, and both processes loaded 
moderately on the Behavioral factor and had near zero loadings on the Experiential factor. This modified model is presented in Figure 6-2 and consists of seven processes loading on the Behavioral factor and three on the Experiential factor. Item loadings were only minimally different from the Correlated Model and all remained at or above .50. They are presented in Table 6-4. The fit indices for the final Two-factor Hierarchical Model was IFI2 $=.835$, $\mathrm{RMS}=.051$.

The One Factor Hierarchical Model had an initial fit of IFI2 $=.812$, RMS $=.054$, which is inferior to the Two Factor Hierarchical Model. Model statistics showed that substantial model improvements could be made by allowing Consciousness Raising, Dramatic Relief, and Environmental Reevaluation to correlate with each other. This model, with these correlations added, is essentially equivalent to the Two Factor Hierarchical Model in both structure and fit.

The Two Factor Hierarchical Model fits the data less well than the Correlated Model. This is expected because the Hierarchical Model is more parsimonious in design. The fully correlated model has 45 correlations that are freed to be estimated, whereas the Hierarchical Model has ten loadings and one correlation between the higher order factors. One way of adjusting for this difference in model design is to create parsimonious fit indices by multiplying the original fit indices by the ratio of degrees of freedom of the Null Model to the degrees of freedom in the models in question (Mulaik, James, Van Alstine, Bennett, Lind, \& Stilweli, 1988). These parsimonious fit indices are not designed to be compared to absolute criteria but are used to compare models to each other. The results show that these models have nearly identical parsimonious fits indices (Correlated Model: $\operatorname{IFI} 2$ (parsimonious) $=.785$; Two Factor Hierarchical Model: IFI2 (parsimonious)=.779). The Two Factor Hierarchical Model was chosen for further exploration because of its superior heuristic value and its closeness to previous model findings. 


\section{The Processes of Resistance}

\section{Exploratory Factor Analysis}

Subjects were eliminated from this analysis for two reasons. First, cases that had missing data on more than two of the 22 items were eliminated ( $5 \%$ of the sample). Second, cases which had a mean response across all 22 items of 1.5 or less were eliminated (10\% of the remaining sample). This last criterion was chosen to eliminate subjects that had very low endorsement of items. Cases with such an extremity response bias are not useful in the exploration of the dimensionality of an item set and can inflate component inter-correlations (Jackson, 1970; Velicer et al, 1990). The subjects that were removed were predominantly in the stage of Precontemplation for Acquisition (55\% of the removed subjects) and Maintenance for Cessation (20\%).

The remaining sample was randomly split into two subsamples. An exploratory principal component analysis was conducted on the $22 \times 22$ matrix of item inter-correlations generated using pair-wise deletion $(\underline{N}=295)$. The number of components to retain was determined by using the results of three procedures that have been shown to be valid predictors of the correct dimensionality of an item set (Zwick \& Velicer, 1986). These are the Scree procedure (Catell, 1976), the Minimum Average Partial procedure (Velicer, 1976), and Parallel Analysis (Horn, 1968; Lautenschlager, 1989). All three procedures indicated that three was the correct number of components to extract. Both orthogonal (varimax) and oblique (direct quartimin) rotations were performed on a three component solution. As these solutions produced virtually identical results, the varimax rotation was chosen for further analysis.

Items were eliminated from the analysis if they did not load highly on any of the components (unique items), loaded highly on more than one component (complex items), or loaded highly on a component that they were not hypothesized to load on. Ideally a final item set will consist only of items with high loadings on the component they were hypothesized to measure and low loadings on the other components (Jackson, 1970, 1971). After items were 
removed for the above reasons, the analysis was rerun, and the loading pattern re-examined. In this analysis, item elimination was stopped when scales were composed of only simple items or when the scale was reduced to four items. This second criterion was chosen because scales of less than four items tend to be unstable and unreliable. More information on itemcomponent relationships was obtained during the confirmatory factor analysis (see below).

The final solution consisted of 15 items and three components. These components were interpreted as scales measuring the three hypothesized Processes of Resistance. The first scale, Rationalization, was composed of 6 items. This scale explained $23.6 \%$ of the unrotated variance of the original item set and had a Coefficient Alpha for Sample 1 of .75 . The second scale, Reactance, was composed of 5 items. It explained $7.9 \%$ of the original, unrotated variance and had a Coefficient Alpha of .73. The third scale, Repression/Denial was composed of 4 items. One item was somewhat complex, with a loading of .41 on this scale and .38 on the Rationalization scale. The Repression/Denial scale explained $6.3 \%$ of the variance. The Coefficient Alpha was .53, which indicates a low internal consistency. These three components explained $46.7 \%$ of the variance for the reduced item set. Final component loadings are presented in Table 6-6.

\section{Confirmatory Factor Analysis}

Confirmatory factor analysis using the EQS structural modeling program (Bentler, 1989 ) was performed using the hold out sample $(\mathrm{N}=241)$. Three models were run. The null model, which posits 15 independent variables, is not expected to fit the data but generates a set of statistics to which the other models can be compared. The second was a uncorrelated three factor model and the third was a correlated three factor model.

A set of fit indices including Chi square statistic, Comparative Fit Index (CFI), NonNormed Fit Index (NNFI), Root Mean Square (RMS), and Incremental Fit Index, type 2 (IFI2) were calculated to assess model fit. Since these indices all produce a similar pattern of 
results, only the IFI2 is presented here. These fit indices indicated that the correlated three factor model was superior to the others $(\mathrm{IFI} 2=.91)$. This model is presented in Figure 6-3.

Although model fit indices were acceptable for this model factor loadings for two of the Repression/Denial items were very low at .30. Furthermore the Coefficient Alpha for this scale for sample 2 was .48 , even lower than for Sample 1. Because of these considerations a two factor, correlated model was also assessed. This model had similar model fit indices as the correlated three factor model and a virtually identical factor loading pattern on the two retained factors, as did the original three factor model. In both of these models two Reactance items have marginally low loadings of .39. The two factor model is presented in Figure 6-4.

Scale scores were calculated by taking an unweighted mean of item responses for subjects who had answered at least $50 \%$ of the respective scale's items. Scale means, standard deviations and internal consistencies are presented in Table 6-7.

These results suggests that both the Rationalization scale and the Reactance scale have good psychometric properties, but further development could improve average item saturation. The Repression/Denial scale has poor item saturation and internal reliability. Further conceptual and empirical development is necessary to improve this scale's psychometric properties.

\section{Combined Processes of Change and Processes of Resistance Models}

Having developed the Processes of Change and the Processes of Resistance instruments separately, these instruments were then combined in one structural model to assess their relation to each other. Three models were fit to the data. The Null Model was run to generate statistics with which to compare the other models. The second model was a Correlated Hierarchical Model which allowed the second order factors of Experiential Processes, Behavioral Processes, and Processes of Resistance to correlate with each other. The third model was a Third-order Hierarchical Model with the Experiential and Behavioral 
factors loading on a third order Process Factor which was allowed to correlate with the second order Resistance factor. After initial model fit, indicators suggesting model modifications were examined and modifications were made.

The initial model fit for both substantive models was identical with IFI $2=.79$ and RMS $=.056$. These numbers represent marginal model fit. Modification indices suggested that the processes of Self Reevaluation and Stimulus Control were complex with secondary loadings on the Process of Resistance factor. These loadings are of similar magnitude (between .30 and .40 ) but of opposite direction with Self Reevaluation loading positively and Stimulus Control loading negatively on the Resistance factor. Furthermore, the correlations between the Resistance factor and the Behavioral factor $(r=.06)$ was insignificant as was the correlation in the third model between the third order Process factor and the Resistance factor $(r=.04)$. These correlations were removed from the models.

The resulting models are presented in Figures 6-5 and 6-6. Mathematically it is only the correlation between the Experiential and Resistance factors that differentiates these models, and this correlation is small and has little effect on overall model fit. The models have nearly identical first and second order factor loadings and fit indices (Correlated Model: $\mathrm{IFI} 2=.804, \mathrm{RMS}=.0534$; Hierarchical model: $\mathrm{IFI} 2=.803, \mathrm{RMS}=.0532$ ). The choice between these two is a choice between alternate conceptualizations of the relationship among these three types of processes. The Experiential and Behavioral Processes of Change were originally conceived as being two types of processes which promote behavior change and the Processes of Resistance as mental activities that resist change pressures. Therefore it is thought that the Hierarchical Model most clearly captures this conceptualization. The high loadings of the Experiential and Behavioral processes on the Process factor also supports this conceptualization. Although it was expected that the Processes of Resistance would be negatively correlated with the Processes of Change this was largely not the case. In the Correlated Model, there was only a small negative correlation between the Resistance and Experiential factors $(r=-.12)$. These types of processes are largely independent. 
As a check on the effect of scale length on model fit, a model with the same higher order structure as the Hierarchical Model was run with item parcels. Items for scales with more than three items were randomly combined into two-item parcels to create three manifest indicators for each process. This model showed improved fit indices of IFI $2=.88$ and RMS $=.051$. These figures support the use of this model as fitting the data satisfactorily. Factor loadings were similar to the Hierarchical Model.

As a further assessment of the relationship among the Processes of Change and the Processes of Resistance, Pearson correlations were calculated and are presented in Table 6-8. The correlations between Self Reevaluation and Stimulus Control and the Processes of Resistance parallel the complex loadings found in the structural models. Other findings suggest that Helping Relationship is positively associated with the processes of Resistance and that Consciousness Raising and Environmental Reevaluation are negatively associated with these processes.

\section{EXTERNAL VALIDITY}

To assess external validity of the Process scales, their relationship to a number of variables was examined. Pearson correlations were calculated for the processes with age, three alcohol consumption variables, three scales measuring alcohol related experiences, and scales that compose the Decisional Balance, Temptation, and Stage of Change constructs of the Transtheoretical Model. In addition, analysis of variance techniques were used to investigate the relationship of the Process scales to both gender and the algorithmic measure of the Stage of Change. This set of variables provides information on the relationship of Process scales to an important demographic variable for a still maturing population (age), three variables that are measures of the behavior being examined, three measures of the risks associated with this behavior, and a set of variables that will assess the application of the Transtheoretical model to this behavioral domain (model validity). The correlations with age 
and the alcohol related variables are presented in Table 6-9 and the model variables in Table 6-10.

\section{Continuous Variables}

An examination of the large number of correlations calculated reveals clear patterns. First, age had remarkable consistent low negative non-significant correlations for 10 of the 13 processes. Two processes, Environmental Reevaluation and Self Monitoring, had small nonsignificant positive correlations. The only significant correlation was for the Reactance scale $(r=-.19)$. This is consistent with the nature of the scale which measures one's resistance to being coerced, something which is associated with young age, and which become much less salient for students who have reached the legal drinking age of 21 . Second, there were sets of validating variables that had very similar patterns of correlations across the Processes. These sets include the consumption variables (Days, Drinks, and Intox) and the Excess scale, with the Intox scale having generally lower correlations. The Distress and Problem scales also had similar correlations, with those of the Problem scale generally being of a lower magnitude. For the Transtheoretical Model variables, Temptations and the Pros had similar correlations, as did the two Cons scales.

There were three basic patterns of process correlations across the validating variables. The first is exemplified by the processes of Environmental Reevaluation, Consciousness Raising, and Stimulus Control. These variables have negative correlations with the alcohol consumption variables, the Alcohol-Related Experience Scales (most strongly with Excess), the Precontemplation, Temptation, and Pros scales. They have moderate positive correlations with the two Cons scales and the Contemplation scale and small positive correlations with the Maintenance scale. This suggests that subjects who use these processes tend to drink less, to have fewer negative consequences of alcohol, to not think heavy drinking is acceptable or beneficial or be tempted to do so. They are more aware of the negative effects of alcohol and are considering reducing their own drinking habits. 
Other processes have a pattern of correlations similar to this pattern, with some small differences of interest. Dramatic Relief has this type of pattern but with no relationship to Temptation or the Pros. Self Monitoring also has non-significant correlations with Temptation and Pros and no relation to the Contemplation scale, but exhibits the strongest relation of all the processes to the Maintenance scale suggesting that those who use this process are likely to be in a late Stage of Change and are resolved about their drinking. The processes of Reinforcement Management, Counter-Conditioning, and Self Liberation tend to have smaller or non-significant negative correlations with the consumption variables and Excess, Temptations and Pros whereas their correlation with the Contemplation scale is stronger. This suggests that these processes are less associated with lower alcohol consumption but more associated with uncertainty about their drinking habits.

The second pattern is seen with the process of Self Reevaluation. This process has significant positive correlations to 11 of the 13 validity variables, many of which are large. It has small to moderate positive correlations with the consumption variables, has the strongest correlations of all the processes with the Distress and Problem scales $\underline{r}=.45$ and .33 respectively), and has positive correlations with the Temptation and Pros scales, but also has strong positive correlations to the Cons and the Contemplation scale. Its two non-significant correlations are small negative correlations with the Precontemplation and Maintenance scales. This pattern of results suggests that those that use this process are in conflict, being both tempted to drink and recognizing the negative results of drinking and they are strongly considering changing their drinking habits. This characterization is consistent with the nature of this process.

Helping Relationship exhibited a similar pattern but with much smaller correlations. It has small positive correlations with all thirteen variables, four of which were nonsignificant. Talking to another about one's drinking implies that one has some concerns about one's drinking which therefore is unlikely to be at low levels. This might account for the 
positive correlations across the validating variables, but these are smatl correlations that should not be over-interpreted.

The third pattern of correlations are exemplified by the Processes of Reactance and Rationalization. These variables have largely the opposite correlations of the first pattern. Those who use these processes tend to drink more, suffer more alcohol-related consequences, be accepting of heavy drinking, value the benefits of drinking, and be tempted to drink. These processes have small positive correlations with the Contemplation scale and have negative correlations to the Maintenance scale. Repression/Denial has a similar pattern, but its correlations tend to be smaller (six of the thirteen correlations are non-significant at $\mathrm{p}<.01$ ) which might be related to this scale's low reliability. These correlations largely fit the hypothesized attributes of the Processes of Resistance although the small positive correlations with the Contemplation Scale suggest that those who use these processes are in more conflict about their drinking than might be expected.

\section{Stage of Change}

The relationship between the Processes and Stage of Change was also examined. The ten Processes of Change and the three Processes of Resistance were entered into a two-way Stage by gender MANOVA. The main effect of Stage proved significant $(\Lambda(52,1508.7)=.586, \underline{\mathrm{p}}<.0001)$, explaining $41 \%$ of the variance. The main effect of gender was also significant $(\Lambda(13,389)=.852, \mathrm{~g}<.0001)$ explaining $15 \%$ of the variance. The interaction did not prove significant $(\Lambda(52,1508.7)=.888, \underline{p}>.05))$.

Follow-up univariate analyses of variance were conducted for each of the thirteen processes for the effect of Stage of Change. Results along with scale means and standard deviations by Stage are reported in Table 6-11. Additionally, scale scores were converted to T-scores $\underline{(M}=50, \underline{S D}=10)$ and means by Stage plotted and presented in Figures 6-7 through 6-11. The effect of Stage was significant $(\mathrm{p}<.05)$ for all processes except Helping Relationship. A cursory review of scores for this process by Stage suggests that this is 
because of the unusually large size of its standard deviations within stage. The effect sizes ranged from .016 to .227 and had a mean of .064, a moderate effect size (Cohen, 1977). For eight of the nine significant Processes of Change, Precontemplation was significantly lower than the peak stage at $\underline{\mathrm{g}}<.05$ and for the ninth, Counter Conditioning, this is true at $\underline{\mathrm{L}}<.10$.

A review of the figures suggests that there are four pattern types. The first pattern is exhibited by Dramatic Relief, Consciousness Raising, Reinforcement Management, and Counter-Conditioning and entails increasing levels of process use through Preparation, a decrease in Action, and an increase to about Preparation levels in Maintenance. The second pattern entails a sharp increase in process use from Precontemplation to Preparation, with an equally sharp decrease to Action, and no change to Maintenance. The two processes with this pattern, Self Liberation and Self Reevaluation, have been shown to be middle stage processes (Prochaska et al., 1991). The third pattern is seen for Environmental Reevaluation, Self Monitoring, and Stimulus Control. For these processes there are relatively small increases between Precontemplation and Action (more so for Stimulus Control) and then a sharp increase for Maintenance. The last pattern is exhibited for the Processes of Resistance and entails generally decreasing process use with stage progression. For Reactance and Rationalization there is little change between Precontemplation and Preparation, and then Iarge decreases to Action and Maintenance. For Repression/Denial there is a moderate decrease in Preparation a slight increase to Action, before a sharp decrease to Maintenance.

A generally consistent finding in these profiles is the relatively low level of Process of Change use observed for the Action stage. In the first pattern described above, the decrease in Action compared to both Preparation and Maintenance is clearly not hypothesized. For Self Reevaluation and Self Liberation the level in Action is equal to the low levels in Maintenance, and not in between the values found for Preparation and Maintenance as would be more expected. And it is also unexpected that for the processes that exhibited a large increase in Maintenance such as Environmental Reevaluation, Stimulus Control, and Self Monitoring there was not a substantial increase in Action also, since both of these stages 
entail behavior change. The low level of process use in Action seen across a majority of the Processes of Change suggests that the Action stage, as composed in this study, is not of the same character as found in previous research. This finding is further investigated below.

\section{Stage of Change with intentional criteria for Action and Maintenance}

The anomalous low level of process use for the Action stage was investigated in a post-hoc fashion. The staging criterion for Action was reviewed. The original staging algorithm used only the traditional behavioral criterion of having stopped immoderate drinking within the last six months. Because the Stages of Acquisition were also measured with the survey instrument, the intention of those in Action and Maintenance to drink immoderately in the future was also assessed. To investigate whether intentional status of those in Action and Maintenance affected process use, subjects who had discontinued immoderate drinking, but intended to resume this drinking pattern in the future were removed from the Action and Maintenance groups. This resulted in the removal of 15 subjects from the Action stage (35\%) and 7 subjects from the Maintenance stage $(9 \%)$. The analysis of variance was then repeated.

The results of the MANOVA were similar to the original analysis, although the percent of variance explained by Stage increased from $41 \%$ to $47 \%(\Lambda(52,1431,24)=.534$, $\mathrm{p}<.0001$ ), suggesting that the stricter criteria created more explanatory classification of subjects. Similarly, ANOVA results showed that the same nine Processes were significant. The $\mathrm{F}$ values and $\omega^{2}$ values increased for all the Processes of Change and Resistance except for Self Reevaluation. The mean effect size was .077 . These results are presented in Table 6-12. Graphs of Process T-scores by Stage were plotted in Figures 6-13 through 6-18. The values for the Action stage increased across the Processes of Change by an average of $2.3 \mathrm{~T}$ points with Consciousness Raising showing the largest increase (3.7 T-points) and Helping Relationship showing the smallest increase (.33 T-points). The scores for the Maintenance stage also increased on all but the Helping Relationship process which showed a small decrease (.33 T-points). Increases were smaller for Maintenance, averaging .8 T-points. 
There was a substantial change for the Processes of Resistance as well, but in the opposite direction. The value at Action decreased between 2.2 and $3.6 \mathrm{~T}$-points for the three scales. Changes for the Maintenance stage were generally small for the Processes of Resistance.

These changes produced a set of patterns that are qualitatively different than the original patterns found. The new patterns show a near linear change in Process of Change use between Preparation and Maintenance for all but Self Reevaluation and Dramatic Relief. The Action group continues to use Self Reevaluation at values closer to Maintenance than to Preparation, but at somewhat elevated values from the original Action stage. The pattern for Dramatic Relief continues to show a dip in use in Action relative to both Preparation and Maintenance, although the magnitude of this decrease is about half that previously measured.

The new patterns show that, as for the original Stages, seven of the nine significant Processes of Change exhibit increases in process use in Contemplation or Preparation. These can be separated into processes that roughly maintain this level in Action and Maintenance (Counter Conditioning and Reinforcement Management, with Dramatic Relief showing the above mentioned dip in the Action stage), processes that show a decrease in Action and Maintenance (Self Reevaluation and Self Liberation), or those that continue to show increases in the last two stages (Consciousness Raising and Stimulus Control). The other two processes do not show increases in the early stages but sharp increases in Action and in Maintenance (Environmental Reevaluation and Self Monitoring).

The major qualitative change in the Processes of Resistance is that there is no longer an increase in use of Repression/Denial between Preparation and Action, although the flat pattern now observed between these two stages remains in sharp contrast to the substantial decreases in Action for the other two Processes of Resistance (see Figure 6-18).

The pattern for Environmental Reevaluation is most divergent from what was hypothesized, as this process has been seen as an early stage process. The late stage increases for Consciousness Raising were also unexpected, although the early increase in Contemplation 
was predicted. The pattern seen for Self Monitoring, a new process, confirms that this process is most related to the reduction to and the maintenance of moderate drinking.

Overall the relationships of the Processes of Change and Resistance to the revised Stage groups were stronger and followed hypothesized patterns more closely. These findings suggest that the addition of an intentional criterion for the Action and Maintenance stages results in a more accurate staging algorithm. This result is based on a post-hoc revision which will need to be replicated in an independent sample before it is taken as an established result for immoderate drinking in college.

These results also define a new group, namely those who have decreased their drinking to moderate levels but plan to return to immoderate drinking in the near or moderate future. Those in this group ( $n=22,3.5 \%$ of the total sample) use the Processes of Change much less and the Processes of Resistance much more than others who have reduced their drinking but who do not intend to return to immoderate drinking in the future.

A summary of important changes in process use by stage is presented in Table 6-13. To further present the relationship of the Processes of Change and the Processes of Resistance across the Stages of Change (using the revised Stage groups) the nine significant Processes of Change are plotted against Rationalization in Figures 6-18 to 6-20. Rationalization was chosen as the most representative and psychometrically sound Process of Resistance. As can be seen from these figures Rationalization is clearly at higher values than all the Processes of Change in Precontemplation, is less than or in close balance with seven of these processes in Preparation, and is much lower than all these processes in Maintenance. This suggests that stage progression entails a total reordering of process use with an approximate balance between the Processes of Change and of Resistance in the middle stages of Contemplation and Preparation. 


\section{Gender and Process use}

Follow-up univariate analyses of variance for gender were conducted for all thirteen processes and six were statistically significant. Means by gender along with the ANOVA results are provided in Table 6-14. Women used two of the processes, Dramatic Relief and Self Monitoring, significantly more than the men, and men used Reinforcement Management, Counter-Conditioning, Self Reevaluation, and Reactance more than the women. Some of these results follow what would be expected from gender role stereotypes. Women are seen as more emotionally reactive and men as more rebellious. Women's higher use of Self Monitoring might be somewhat due to their lower biological tolerance (Frezza, De Padova, Pozzato, Terpin, Baraona, \& Lieber, 1990), which makes being aware of alcohol intake and its effects more important. 


\section{DISCUSSION}

This study presents strong evidence that the Processes of Change and Resistance are meaningful to the study of immoderate drinking by college students. Model based predictions were largely confirmed, supporting the application of the Transtheoretical Model of Change to this behavior. Exceptions to model predictions were minor, and provide useful information on the nature of the measurement scales and how the change process for immoderate drinking by college students might differ from other behaviors previously investigated with the Transtheoretical Model.

Additionally the results of this study strongly support the reliability and validity of the Processes of Change and Resistance instrument. Of the fifteen processes that were originally hypothesized, thirteen were measured, including ten Processes of Change and three Processes of Resistance. In general, these scales had satisfactory psychometric properties. The weakest scale was Repression/Denial which could use further scale development to increase internal consistency and item saturation. Four other scales had less than optimal internal consistencies: Environmental Reevaluation, Self Liberation, Reinforcement Management, and Counter Conditioning. Validity evidence was found for all five of these scales suggesting that they are tapping important phenomena and that further scale development to improve their psychometrics is warranted.

Of the fifteen originally hypothesized processes, one process, Social Liberation, did not maintain its integrity as a separate scale during exploratory analyses. Although this process has generally been found for other behaviors and with sober alcoholics, it has not proven useful in the design of model based interventions. Two other scales, Stimulus Control and Interpersonal Systems Control, were not found to be independent of each other and were combined into one scale, labeled Stimulus Control. These scales are theoretically related and their combination is not inconsistent with model findings. They were also found to combine in research on cocaine use (Rosenbloom, 1991). It is of note that most of the items in the 
final scale assess interpersonal cues suggesting that although college students do not meaningfully differentiate between these types of cues, their drinking is strongly affected by those around them.

Empirical findings supported an organization of these processes into three higherorder factors, labeled Experiential, Behavioral, and Resistance, and the first two of these factors as a third-order Process of Change factor. Although mathematically a second-order factor (two levels above manifest measures), the Resistance factor is best conceptualized as on the same level as the Process of Change factor, and without a second-order subgrouping structure. The Resistance factor was found to be largely independent of all the other hierarchical factors, but two Processes of Change, Self Reevaluation and Stimulus Control, had small but meaningful relationships with this factor.

There was one minor deviation in the predictions of how the Processes of Change would load on the Experiential and Behavioral factors. Self Reevaluation has been found in previous research to be an Experiential process. The Experiential/Behavioral distinction is thought to reflect the use of processes by early versus late stage members. Self Reevaluation is seen as a middle stage process and its grouping with the Behavioral processes is not a serious deviation from the model. Other behavioral domains have had other deviations from the original grouping pattern, and these have not been seen as a challenge to model integrity (e.g. Redding, 1993; Rossi \& Rossi, 1993).

Self Monitoring is a process not previously investigated within the Transtheoretical Model, but is taken from the literature on controlled drinking, specifically from strategies designed to assist heavy drinkers to establish controlled drinking habits (Miller, 1987). This process loaded with the Behavioral processes and had a strong increases in Action and Maintenance, suggesting that it is heavily associated with the establishment of controlled drinking. Longitudinal research will be necessary to determine the role of this process in behavior change, but these results suggest that it is likely to be an important addition to the set of Processes of Change for this behavior. Furthermore this process is likely to be 
applicable to other behavioral domains that entail reduction, but not elimination, of behaviors such as changing dietary intake. Another possible area of application, although more distal from controlled drinking, is the acquisition of healthy behaviors such as exercise or stress reduction activities.

The Processes of Resistance that were developed in this study represent a new development in the Transtheoretical Model. Although similar attitudes have been previously investigated in the area of weight control (Prochaska, Norcross, Fowler, Follick, \& Abrams, 1992), they have not been systematically incorporated into this model. Although, as mentioned above, one of the processes, Repression/Denial, had poor psychometrics, all three Processes of Resistance had clear external validity evidence and adequate loadings on the Resistance factor. Evidence suggests that these processes are not just the opposite of the Processes of Change. They do not have meaningful negative correlations with the other higher order factors or with most of the individual Processes of Change. More accurately, the empirical findings support their original conception as processes that individuals use to resist pressures to change.

Shaffer (1990) suggests that the stage of Contemplation is a stage of increasing ambivalence, in which new attitudes are being recognized, increasing internal conflict. The fact that the Processes of Resistance do not significantly decrease during the first three Stages of Change while many of the Processes of Change are increasing, supports this view. It can also be seen in Figures 6-19, 6-20, and 6-21 that a rough balance of the Processes of Resistance and of Change exist in the Contemplation and Preparation stages. It is only with resolution of ambivalence and the movement to Action that these Processes of Resistance significantly decrease. Also supporting this view is the positive correlation with Self Reevaluation, a process that is both a reflection of and an attempt to resolve internal conflict. Of course without longitudinal data other explanations are plausible, such as that the decrease in the use of the Processes of Resistance is a rationalization of Action already taken instead of preceding it. 
In general, the results of this study support the validity of the Processes of Change and Resistance scales, but some of the findings warrant comment. The low correlations of the behavioral processes of Self Liberation, Counter Conditioning, and Reinforcement Management to the outcome variables might be explained as partially an artifact of measuring these processes in the context of controlled use rather than abstinence. The items that compose these scales tend to assess activities related to drinking less or more than at other times and therefore these items will be less salient for those who abstain or those who have attained consistent moderate drinking. For example the Reinforcement Management item "I punish myself for drinking too much" is unlikely to be a behavior of someone who is a consistent light drinker or an abstainer. This result does not imply that these processes are ineffective in helping students reduce their immoderate drinking.

One clear anomalous finding is the lower than expected use of many of the Processes of Change for those in Action when Action is defined with traditional behavioral criteria. The discovery that there is a subgroup of those in Action and Maintenance who intended to return to immoderate drinking that accounts for this finding raises a number of important questions. First and foremost is whether this is a robust finding. As it was the result of a post-hoc exploration to explain an anomalous finding there is a chance that it is the result of sample characteristics, and does not represent a phenomenon found in the total population. Replication of this finding would be important before it is concluded that staging criteria that have been validated across a wide range of behaviors and populations need to be changed.

Assuming that this is a robust finding, the next important question is what is the motivation of students to both give up immoderate drinking and intend to return to it. There are a number of possibilities. It might be that some students with unstable drinking habits "unintentionally" slip into Action by not drinking immoderately at least once a week for a period of time, while recognizing their intention to do so in the future. Alternatively, students might have overt time limited reasons for reducing their drinking, such as participation in athletics, needing to improve their school performance, or being on a time limited probation. 
Students might have other more covert reasons as well, such as wanting to experiment with lower drinking for a while or to test their will power. Another possible explanation of this finding is that it is an overlap of Acquisition and Cessation phenomena, that for some students their previous immoderate drinking was an experiment in a process of establishing the habit, from which they are taking a break, but plan to return in the future. Further research will be necessary to determine the motivations of these students.

The relationships of the Processes of Change to the revised stage variable are of interest. In general there was a high use of processes in Action and Maintenance, with only Self Reevaluation and Self Liberation showing reductions, and four processes, Environmental Reevaluation, Consciousness Raising, Stimulus Control, and Self Monitoring, showing peaks in the Maintenance stage. This is in contrast to the patterns found for smoking, where experiential processes tended to peak in Preparation and the behavioral process in Action. This is more similar to what has been discovered for the acquisition of low fat diets (Rossi \& Rossi, 1993). These findings suggest that the reduction of a behavior, without the elimination of it, requires more activity for longer than the total cessation of a behavior. For college drinking, the heavy use of processes might reflect the difficulty of maintaining a moderate level of drinking without the total extinction of cues that can occur in abstinence.

Alternatively it might evidence the difficulty of maintaining moderate drinking in a heavy drinking environment.

The relationship of two of the Processes of Change to Stage deviated most from what was hypothesized. The first is Environmental Reevaluation, which showed very little increase at all until the Action stage. Previously this has been found to be an early stage process (Prochaska et al., 1991). An examination of the items in this scale suggests that they are measuring an attitude of concern for the negative effects of alcohol use on society. This is consistent with how Environmental Reevaluation is conceptualized but is likely to also reflect a clear anti-drinking attitude. Although similar items produced an early stage process for smoking (Prochaska et al., 1991), for college drinking it seems that this attitude does not 
coexist with ambivalence about immoderate drinking. In the diet area, Environmental Reevaluation also peaks in Maintenance (Rossi \& Rossi, 1993). Perhaps these results indicate how this process is used in attempts to control a behavior rather than eliminate it.

Alternatively, items measuring other aspects of Environmental Reevaluation might be more salient for this population and behavior. For example, items constructed to measure this process for exercise acquisition ask about subjects' consideration of the effect that positive behavior change would have on others in their immediate environment (Marcus et al., 1992 ). Items include "I wonder how my inactivity affects those people who are close to me". These items could easily be adapted for immoderate drinking, and might measure an aspect of Environmental Reevaluation that is more salient to college students and more effective in promoting early stage progress.

Helping Relationship is the only process that did not have a significant relationship to Stage of Change. This process was originally based on the Rogerian or client-centered therapy paradigm and entails the use of unconditional acceptance of others to promote positive behavior change (Prochaska, 1979). It is therefore true that in a behavioral domain where there are high levels of ambivalence both within individuals and within the societal context of college students, this process can be used in many ways, including receiving support for one's heavy drinking. Although the original item set included items that did not preserve neutrality about drinking habits, the three items that remained in the scale did so. This result might explain the lack of relationship to stage and this scale's high mean and large standard deviation. Including more items that assess only the help others provide to reduce drinking might result in a more useful scale.

The relationships of these variables to the Stages of Change not only validate the scales, but characterize the stages as well and have implications for interventions to reduce immoderate drinking. Interventions based on the Transtheoretical Model would subordinate the goal of behavior change to that of moving students to the next stage of change. This strategy is based on previous research that has demonstrated for other behaviors that this is 
the most effective way to promote permanent behavior change (Prochaska, DiClemente, Velicer, \& Rossi, 1993). This is different than traditional intervention programs that largely focus on behavior change. Although it is unlikely that these programs fail for any simple reason, the premature pushing of Precontemplators and Contemplators into Action might be one cause.

It has been found that successful change efforts make differential use of the processes across the stages and that interventions can be designed to effectively promote stage appropriate process use to change and maintain positive behavior change (Prochaska et al., $1991 ; 1993)$. The cross-sectional results of the present study suggest that for immoderate alcohol use, movement from Precontemplation to Contemplation entails an increase in many of the Processes of Change, but most especially the increased use of Consciousness Raising, Self Reevaluation, Counter Conditioning, and Reinforcement Management. Further increases in Self Reevaluation, Dramatic Relief, and Self Liberation would be useful to help Contemplators move to Preparation. The Processes of Resistance do not show a clear pattern of decrease during the first three stages which would suggest that interventions that confront resistance directly and early might not be useful for college students. This suggestion conflicts with many traditional intervention protocols.

Interventions for those who take Action and are trying to maintain reduced drinking would target increasing the use of Stimulus Control, Environmental Reevaluation, and Self Monitoring along with decreasing the use of the three Processes of Resistance. There are also large decreases in the use of Self Reevaluation and Self Liberation between Preparation and Action. It has been shown previously that the continued use of Self Reevaluation in the Action stage predicts relapse (Prochaska et al., 1993), suggesting that helping students not reevaluate after they have made a decision to reduce their drinking would be useful. Making the same recommendation with confidence for Self Liberation in the absence of similar research findings awaits further research. The high levels of process use in Maintenance 
suggests that informed support for an extended length of time might be necessary to reduce relapse rates.

A further issue is what type of intervention would be most effective for those students who have reduced their drinking but intend to resume immoderate drinking in the future, those who were removed from the Action and Maintenance stages. Since intention is usually a very strong predictor of future behavior, it is likely that these students will drink immoderately again. An intervention designed as a relapse prevention program might be-most appropriate. The specifics of this program will depend somewhat on the answers to the questions posed above, namely why did these students stop their immoderate drinking and why are they planning to resume it. If it is because of a changing situation, in other words the students realize that the Pros and Cons of their drinking will change, then a focus on these variables might be most useful. If it is more a testing of will power, then perhaps helping them with their felt temptation to drink is most appropriate. Use of the Processes of Change can be employed in both of these intervention schemas, with processes such as Environmental Reevaluation and Consciousness Raising being more likely to affect Decisional Balance variables, and the behavioral Processes of Reinforcement Management, Counter Conditioning, and Stimulus Control being used to modify temptations.

The results of this study need to be interpreted with appropriate caution. Although cross-sectional data across the Stages of Change have tended to replicate longitudinally, this will have to be demonstrated in this behavioral domain. Furthermore the sample used was neither representative within the university chosen, nor was the university necessarily representative of all colleges and universities. The large sample size increases the potential for these results to replicate for this university, and it is also likely that these results will be most applicable to large, state universities, where college drinking is prevalent. 


\section{REFERENCES}

Allen, S. J. \& Hubbard, R. (1986). Regression equations for the latent roots of random data correlation matrices with unities on the diagonal. Multivariate Behavioral Research, 21, 393-398.

Bentler, P. M. (1989). EQS:Structural equations program manual. Los Angeles, California: BMDP Statistical Software.

Cattell, R. B. (1966). The Scree test for the number of factors. Multivariate Behavioral Research, 1 , 245-276.

Frezza, M., de Padova, C., Pozzato, G., Terpin, M., Baraona, E., \& Lieber, C. S. (1990). High blood alcohol levels in women: The role of decreased gastric alcohol dehydrogenase activity and first-pass metabolism. New England Journal of Medicine, 322, $127-129$.

Engs, R. C. \& Hanson, D. J. (1986). Age-specific alcohol prohibition and college students' drinking problems. Psychological Reports, $\underline{59}$, 979-984.

Harford, T. C. (1984). Situational factors in drinking: A developmental perspective on drinking contexts. In P. M. Miller \& T. D. Nirenberg (Eds.), Prevention of alcohol abuse (pp. 119-156). New York: Plenum Press.

Horn, J. L. (1965). A rationale and test for the number of factors in factor analysis.

Psychometrika, 30, 179-185.

Jackson, D. N. (1970). A sequential system for personality scale development. In Spielberger (Ed.), Current topics in clinical and community psychology (Vol. 2, pp. 61-96). New York: Academic Press.

Kraft, D. P. (1988). The prevention and treatment of alcohol problems on a college campus. Journal of Alcohol and Drug Education, 34, 37-51. 
Lautenschlager, G. J. (1989). A comparison of alternatives to conducting Monte Carlo analyses for determining parallel analysis criteria. Multivariate Behavioral Research, $\underline{24}, 365-395$.

Marcus, B. H., Rossi, J. S., Selby, V. C., Niaura, R. S., \& Abrams, D. B. (1992). The Stages and Processes of Exercise Adoption and Maintenance in a Worksite Sample. Health Psychology, $\underline{11}, 386-395$.

Miller, W. R. (1987). Techniques to modify hazardous drinking patterns. In M. Galanter (Ed.), Recent developments in alcoholism, (Vol. 5. pp. 425-438). New York: Plenum Mulaik, S. A., James, L. R., Van Alstine, J., Bennett, N., Lind, S., \& Stilwell, C. D. (1988). Evaluation of goodness-of-fit indices for structural equation models. $\underline{\text { Psychological Bulletin, } 105}$, 430-445.

Prochaska, J. O. (1979). Systems of psychotherapy: A transtheoretical analysis (2nd ed.). Homewood, Illinois: Dorsey Press.

Prochaska, J. O. \& DiClemente, C. C. (1983). Stages and processes of self-change of smoking: Toward an integrative model of change. Journal of Consulting and Clinical Psychology, 51, 390-395.

Prochaska, J. \& DiClemente, C. (1984). The transtheoretical approach: Crossing the traditional boundaries of therapy. Homewood, Illinois: Dow-Jones/Irwin.

Prochaska, J. O. \& DiClemente, C. C. (1985). Common processes of self-change in smoking, weight control and psychological distress. In S. Shiffman \& T. Wills (Eds.), Coping and substance use: A conceptual framework (pp. 345-363). New York: Academic Press.

Prochaska, J. O., DiClemente, C. C., Velicer, W. F., \& Rossi, J. S. (1993). Standardized, individualized, interactive and personalized self-help programs for stages of smoking cessation. HealthPsychology, 12, 399-405. 
Prochaska, J. O., Norcross, J. C., Fowler, J. L., Follick, M. J., \& Abrams, D. B. (1992). Attendance \& outcome in a worksite weight control program: Processes and stages of change as process and predictor variables. Addictive Behaviors, $\underline{17}, 35-45$.

Prochaska, J. O., Rossi, J. S., \& Snow, M. G. (1992). Processes of Change in Alcoholics Anonymous: Maintenance Factors in Long-Term Sobriety. Journal of Studies on Alcohol.

Prochaska, J. O., Velicer, W. F., DiClemente, C. C., \& Fava, J. (1988). Measuring processes of change: Applications to the cessation of smoking. Journal of Consulting and Clinical Psychology, 56, 520-528.

Prochaska, J. O., Velicer, W. F., Guadagnoli, E., Rossi, J. S., \& DiClemente, C. C. (1991). Patterns of change: Dynamic typology applied to smoking cessation. Multivariate Behavioral Research, 26, 83-107.

Redding, C. A. (1993). The transtheoretical model applied to safer sex behavior among university students: A cross-sectional investigation. Unpublished doctoral dissertation, University of Rhode Island, Kingston, RI.

Rosenbloom, D. (1991). A transtheoretical analysis of change among cocaine users. Unpublished doctoral dissertation, University of Rhode Island, Kingston, RI.

Rossi, J. S. (1992, August). Common process of change across nine problem behaviors. Paper presented at the 100th convention of the American Psychological Association in Washington, DC.

Rossi, J. S., Prochaska, J. O., \& DiClemente, C. C. (1988). Processes of change in heavy and light smokers. Journal of Substance Abuse, $1,1-9$.

Rossi, S. R. \& Rossi, J. S. (1993, March). Processes of Change for Dietary Fat Reduction. Paper presented at the 13th annual convention of the Society for Behavioral Medicine, San Francisco, CA. 
Shaffer, H. J. (1992). The psychology of stage change: The transition from addiction to recovery. In J. H. Lowinson, P. Ruiz, R. B. Millman, \& J. G. Langrod (Eds.), Substance abuse: A comprehensive textbook (2nd ed., pp. 100-105). Baltimore: Williams \& Wilkins.

Snow, M. G. (1991). A transtheoretical analysis of strategies in the recovery process from alcohol problems. Unpublished doctoral dissertation, University of Rhode Island, Kingston, RI.

Stevenson, J., Migneault, J. P., \& Mitchell, R. (1990, November). The University of Rhode Island alcohoI-drug health survey: Final report. Unpublished report.

Vaillant, G. E. (1977). Adaptation to Life. Boston; Little, Brown and Company.

Velicer, W. F. (1976). Determining the number of components from the matrix of partial correlations. Psychometrika, 41, 321-327.

Velicer, W. F., DiClemente, C. C., Rossi, J. S., \& Prochaska, J. O. (1990). Relapse situations and self-efficacy: An integrative model. Addictive Behaviors, 15 , 271-283.

Wechsler, H., Davenport, A., Dowdall, G., Moeykens, B., \& Castillo, S. (1994). Health and behavioral consequences of binge drinking in college. Journal of the American Medical Association, 272, 1672-1677.

Werch, C. E. (1990). Behavioral self-control strategies for deliberately limiting drinking among college students. Addictive Behaviors, 15, 119-128.

Zinberg, N. E. \& Fraser, K. M. (1979). The role of social setting in the prevention and treatment of alcoholism. In J. H. Mendelson \& N. K. Mello (Eds.), The diagnosis and treatment of alcoholism (pp. 457-483). New York: McGraw-Hill.

Zinberg, N. E., Harding, W. M., \& Winkeller, M. (1977). A study of social regulatory mechanisms in controlled illicit drug users. Journal of Drug Issues, $\underline{7}, 117-133$.

Zwick, W. R. \& Velicer, W. F. (1986). Comparison of five rules for determining the number of components to retain. Psychological Bulletin, 99, 432-442. 
Table 6-1: Processes of Change and Resistance definitions

\section{Processes of Change}

Consciousness Raising

Environmental

Reevaluation

Dramatic Relief

Self Reevaluation

Self Liberation

Reinforcement

Management

Counter Conditioning

Helping Relationship

Stimulus Control

Interpersonal Systems

Control

Self Monitoring

Social Liberation

Processes of Resistance

Rationalization

Reactance

Repression/Denial

\section{Definitions}

Efforts by the individual to seek new information and to gain understanding about the problem behavior

Consideration and assessment of how the problem behavior affects the physical and social environment

Affective experience related to problem behavior

Cognitive and emotional reappraisal of one's values as they relate to the problem behavior

Choosing and making commitments to change the problem behavior

Changing contingencies that control or maintain the problem behavior

Substitution of alternative behaviors for the problern behavior

Using the support of caring others in dealing with the problem behavior

Control of environment or situational cues which tend to trigger the problem behavior

Controlling or eliminating interpersonal interactions that could elicit the problem behavior

Increasing and maintaining awareness of the behavioral components of the problem behavior and the feeling states associated with this behavior

Awareness, acceptance and promotion of alternative problem-free lifestyles in society

Intellectual justification of the problem behavior

Reacting with increased motivation to engage in the problem behavior when it is restricted to maintain or increase one's sense of autonomy

The ignoring or minimizing of the extent or the effects of the problem behavior 
Table 6-2: Factor correlations from Correlated Processes of Change structural model

\begin{tabular}{|c|c|c|c|c|c|c|c|c|c|}
\hline & CR & ER & DR & SR & SL & $\mathrm{RM}$ & $\mathrm{CC}$ & HR & SC \\
\hline \multicolumn{10}{|l|}{$\mathrm{CR}$} \\
\hline ER & .87 & & & & & & & & \\
\hline DR & .82 & .79 & & & & & & & \\
\hline SR & .39 & .24 & .51 & & & & & & \\
\hline SL & .70 & .43 & .55 & .58 & & & & & \\
\hline $\mathrm{RM}$ & .74 & .56 & .58 & .68 & .96 & & & & \\
\hline $\mathrm{CC}$ & .74 & .57 & .60 & .58 & .89 & .97 & & & \\
\hline HR & .26 & .12 & .30 & .32 & .43 & .34 & .34 & & \\
\hline SC & .69 & .58 & .56 & .21 & .64 & .72 & .69 & .07 & \\
\hline$S M$ & .30 & .26 & .21 & .03 & .46 & .46 & .30 & .23 & .38 \\
\hline
\end{tabular}

Note: $\mathbf{C R}=$ Consciousness Raising, ER $=$ Environmental Reevaluation, $\mathbf{D R}=$ Dramatic Relief, $\mathbf{S R}=$ Self Reevaluation, $\mathbf{S L}=$ Self Liberation, $\mathbf{R M}=$ Reinforcement Management, $\mathbf{C C}=$ Counter Conditioning, $\mathbf{H R}=$ Helping Relationship, $\mathrm{SC}=$ Stimulus Control, SM $=$ Self Monitoring 
Table 6-3: Means, standard deviations, coefficient alphas for the Processes of Change scales

\begin{tabular}{lcccc}
\hline SCALE & Items & Mean & SD & Alpha \\
\hline Consciousness Raising & 6 & 2.63 & .86 & .79 \\
Environmental Reevaluation & 3 & 2.85 & 1.00 & .68 \\
Dramatic Relief & 6 & 2.77 & .93 & .78 \\
Self Reevaluation & 5 & 1.96 & .80 & .78 \\
Self Liberation & 4 & 2.72 & .96 & .70 \\
Reinforcement Management & 5 & 2.34 & .84 & .70 \\
Counter Conditioning & 3 & 2.41 & .96 & .66 \\
Helping Relationship & 3 & 3.22 & 1.27 & .80 \\
Stimulus Control & 6 & 2.63 & .98 & .86 \\
Self Monitoring & 6 & 3.42 & .88 & .81 \\
\hline
\end{tabular}


Table 6-4: Inter-scale correlations for 10 Processes of Change

\begin{tabular}{|c|c|c|c|c|c|c|c|c|c|}
\hline & $\mathrm{CR}$ & ER & $\mathrm{DR}$ & SR & SL & $\mathbf{R M}$ & $\mathrm{CC}$ & $\mathrm{HR}$ & $\mathrm{SC}$ \\
\hline CR & - & & & & & & & & \\
\hline ER & .65 & - & & & & & & & \\
\hline DR & .67 & .61 & - & & & & & & \\
\hline SR & .35 & .22 & .41 & - & & & & & \\
\hline SL & .56 & .33 & .43 & .45 & - & & & & \\
\hline $\mathrm{RM}$ & .59 & .41 & .47 & .53 & .68 & - & & & \\
\hline $\mathrm{CC}$ & .56 & .39 & .45 & .45 & .64 & .68 & - & & \\
\hline HR & .25 & .10 & .26 & .29 & .36 & .28 & .30 & - & \\
\hline SC & .59 & .47 & .48 & .19 & .50 & .57 & $.53^{\prime}$ & .10 & - \\
\hline SM & .28 & .21 & .20 & .05 & .39 & .36 & .25 & .20 & .35 \\
\hline
\end{tabular}

Note: $\mathbf{C R}=$ Consciousness Raising, $\mathbf{E R}=$ Environmental Reevaluation, $\mathbf{D R}=$ Dramatic Relief, $\mathbf{S R}=$ Self Reevaluation, $\mathbf{S L}=$ Self Liberation, $\mathbf{R M}=$ Reinforcement Management, $\mathrm{CC}=$ Counter Conditioning, $\mathrm{HR}=$ Helping Relationship, $\mathrm{SC}=$ Stimulus Control, SM = Self Monitoring

Correlations $>.11$ are significant at $\mathrm{p}<.01$ 
Table 6-5: Item factor loadings for Hierarchical Structural Model

\section{Consciousness Raising}

I recall information about the benefits of not drinking a lot.

I think about information I have heard about problems associated with drinking too much. $\quad .61$

I look for more information about the effects of alcohol.

I pay attention to articles about the health effects of alcohol. .63

I ask other students about their opinions about đrinking.

I recall information people have given me on the benefits of drinking less.

\section{Environmental Reevaluation}

I wonder about all the ways excessive drinking affects society.

I consider the idea that if people drank less the world would be a better place.

I wonder how much less society's health care cost would be if no-one drank a lot.

\section{Dramatic Relief}

I get upset when I think about the problems drinking causes.

I am emotionally moved when I hear of the harm that excessive drinking has caused. $\quad .54$

Hearing about research on the effects of alcohol use worries me. $\quad .60$

Dramatic portrayals of the evils of đrinking affect me emotionally. $\quad .68$

I react emotionally to the wamings about excessive drinking. $\quad .69$

\section{Self Reevaluation}

I feel that being content with myself means changing my drinking habits.

I struggle with the fact that my drinking habits contradict my changing view of myself. $\quad .66$

The way I drink makes me disappointed in myself.

I wonder about my drinking habits. $\quad .66$

I stop and think that my drinking is causing problems for others. 63

\section{Self Liberation}

I make commitments to myself to drink less.

I use will power to control my drinking.

I tell myself that I will drink less or not at all today.

I remind myself that I am able to reduce the amount I drink. 
Table 6-5 (Cont.): Item factor loadings for Hierarchical Structural Model.

\section{Reinforcement Management}

Other people treat me better when I do not drink too much.

I do something nice for myself for making efforts to drink less.

I punish myself if I drink too much.

I pat myself on the back for limiting my drinking.

Others around me reinforce my not drinking too much. $\quad .56$

\section{Counter-Conditioning}

When I am tempted to have another (or my first) drink I think about or do something else instead.

I find that keeping busy helps me drink less. $\quad .58$

I calm myself down when I get the urge to drink. $\quad .67$

\section{Helping Relationships}

I am open with at least one person I can trust about my drinking. $\quad .66$

I have someone who listens when I need to talk about my drinking.

I have someone I can count on when I am having problems with my drinking. $\quad .74$

\section{Stimulus Control}

I avoid places where there will be a lot of drinking.

I avoid places or events where I tend to drink too much. $\quad .71$

I avoid drinking with people who are heavy drinkers.

I avoid those who tend to push drinks on me.

I try to make friends with people who are not heavy drinkers. $\quad .77$

$\begin{array}{ll}\text { I seek out people who do things that do not involve a lot of drinking. } & .79\end{array}$

\section{Self Monitoring}

I pay close attention to how my body feels when I am drinking.

I stop or slow my drinking down when I start to feel the effects of alcohol.

I keep track of how many drinks I have had when drinking.

I watch how I am acting when drinking. $\quad .67$

I control my drinking by paying attention to how I am feeling. $\quad .60$

I monitor how fast I am drinking. 
Table 6-6: Exploratory component analysis loadings for reduced set of Processes of Resistance items

Component Loadings

Items

I II III

Scale: Rationalization:

1. I think that my drinking is OK because I will drink less after I graduate from college.

$\begin{array}{lll}.74 & -.01 \quad .0\end{array}$

2. I feel that after a hard week at school I deserve to have a good time drinking.

3. I feel that I need the real break from studying or working that drinking can give me.

$\begin{array}{lll}.66 & .30 \quad-.02\end{array}$

4. I think it is natural to drink a lot when you are in college. $\begin{array}{lll}.65 & .04 & .17\end{array}$

5. I think that drinking a lot is just part of growing up. $\begin{array}{lll}.56 & .10 & .22\end{array}$

6. I think that if I did not drink like most others my social life would suffer.

$.26 \quad .14$

\section{Scale: Reactance:}

1. My anger at rules that restrict my right to drink makes me want to drink all the more.

2. Rules saying that I can not drink make me want to drink all the more.

3. Drinking as much as I want makes me feel like my own person.

4. I get enjoyment out of getting away with illegal drinking.

\section{Scale: Repression/Denial:}

1. I try not to worry about my drinking.

2. I think that the bad parts about my drinking are not really that bad.

3. I think that even when I drink a lot it does not hurt anyone. 
Table 6-7: Means, standard deviations, coefficient alphas and scale intercorrelations for the Processes of Resistance scales

Sample $1(N=295)$

\begin{tabular}{lcccccc}
\hline & & & & \multicolumn{3}{c}{ Correlations } \\
\cline { 6 - 7 } SCALE & Mean & SD & Alpha & Ration. & Reactance & Repr./Den. \\
\hline Rationalization & 2.61 & .78 & .75 & - & & \\
Reactance & 2.28 & .82 & .73 & .39 & - &. \\
Repression/Denial & 2.74 & .81 & .53 & .41 & .34 & \\
\hline
\end{tabular}

Sample $2(\mathrm{~N}=241)$

\begin{tabular}{lcccccc}
\hline & & & \multicolumn{3}{c}{ Correlations } \\
\cline { 5 - 6 } SCALE & Mean & SD & Alpha & Ration. & Reactance & Repr./Den. \\
\hline Rationalization & 2.69 & .77 & .72 & - & \\
Reactance & 2.38 & .85 & .73 & .48 & - & \\
Repression/Denial & 2.81 & .79 & .48 & .35 & .20 & - \\
\hline
\end{tabular}

Note: All correlations are significant at $\underline{\underline{D}}<.01$ 
Table 6-8: Scale correlations for the Processes of Change with the Processes of Resistance

\begin{tabular}{llllllllllll}
\hline \multirow{2}{*}{$\begin{array}{c}\text { Processes } \\
\text { of } \\
\text { Resistance }\end{array}$} & CR & ER & DR & SR & SL & RM & CC & HR & SC & SM \\
\cline { 2 - 10 } RD & .02 & -.02 & .05 & .24 & .21 & .11 & .14 & .28 & -.09 & -.15 \\
RE & -.07 & -.11 & .04 & .31 & .02 & .06 & .06 & .18 & -.18 & -.02 \\
RT & -.18 & -.16 & -.05 & .33 & .04 & -.02 & .02 & .21 & -.34 & -.03 \\
\hline
\end{tabular}

Note: $\mathbf{C R}=$ Consciousness Raising, $\mathbf{E R}=$ Environmental Reevaluation, $\mathbf{D R}=$ Dramatic Relief, SR $=$ Self Reevaluation, $\mathbf{S L}=$ Self Liberation, $\mathbf{R M}=$ Reinforcement Management, $\mathbf{C C}=$ Counter Conditioning, $\mathbf{H R}=$ Helping Relationship, $\mathbf{S C}=$ Stimulus Control, $\mathbf{S M}=$ Self Monitoring, $\mathbf{R D}=$ Repression $/$ Denial, $\mathbf{R E}=$ Reactance, $\mathbf{R T}=$ Rationalization

Correlations in bold are significant at $\mathrm{p}<.05$ 
Table 6-9: Correlations of Processes of Change and Resistance with validity variables

\begin{tabular}{|c|c|c|c|c|c|c|c|}
\hline & AGE & DRINKS & DAYS & INTOX & EXCESS & DISTRESS & PROBLEM \\
\hline CR & -.03 & -.30 & -.30 & -.20 & -.31 & -.10 & -.06 \\
\hline ER & .05 & -.30 & -.32 & -.20 & -.32 & -.10 & -.11 \\
\hline $\mathbf{D R}$ & -.08 & -.25 & -.21 & -.21 & -.18 & .05 & -.02 \\
\hline SR & -.06 & .19 & .16 & .12 & .24 & .45 & .33 \\
\hline SL & -.07 & -.09 & -.11 & .03 & -.10 & .10 & .00 \\
\hline $\mathbf{R M}$ & -.07 & -.13 & -.16 & -.04 & -.16 & .09 & .04 \\
\hline $\mathrm{CC}$ & -.03 & -.12 & -.12 & -.01 & -.12 & .04 & .00 \\
\hline HRR & -.05 & .11 & .06 & .05 & .11 & .12 & .04 \\
\hline $\mathrm{SC}$ & -.05 & -.44 & -.42 & -.29 & -.49 & -.20 & -.15 \\
\hline SM & .02 & -.21 & -.12 & -.24 & -.24 & -.14 & -.20 \\
\hline $\mathbf{R D}$ & -.10 & .21 & .23 & .10 & .25 & .17 & .04 \\
\hline $\mathbf{R E}$ & -.19 & .23 & .30 & .15 & .32 & .28 & .30 \\
\hline RT & -.08 & .46 & .46 & .22 & .55 & .40 & .27 \\
\hline
\end{tabular}

Note: $\mathbf{C R}=$ Consciousness Raising, $\mathbf{E R}=$ Environmental Reevaluation, $\mathbf{D R}=$ Dramatic Relief, SR = Self Reevaluation, $\mathbf{S L}=$ Self Liberation, $\mathbf{R M}=$ Reinforcement Management, $\mathrm{CC}=$ Counter Conditioning, HR $=$ Helping Relationship, $\mathrm{SC}=$ Stimulus Control, $\mathrm{SM}=$ Self Monitoring, $\mathbf{R D}=$ Repression $/$ Denial, $\mathbf{R E}=$ Reactance, $\mathbf{R T}=$ Rationalization

Correlations $\geq .11$ in magnitude are significant at $\mathrm{p}<.01$

Correlations $\geq .20$ in magnitude are in bold 
Table 6-10: Correlations of Processes of Change and Resistance with Transtheoretical Model variables

\begin{tabular}{|c|c|c|c|c|c|c|c|}
\hline & PREC & CONT & MAIN & TEMPT & PROS & CONS-P & CONS-A \\
\hline CR & -.39 & .25 & .14 & -.23 & -.15 & .42 & .30 \\
\hline ER & -.40 & .16 & .14 & -.21 & -.16 & .37 & .24 \\
\hline DR & -.38 & .25 & .09 & -.04 & .01 & .41 & .40 \\
\hline SR & -.08 & .58 & -.03 & .31 & .27 & .26 & .43 \\
\hline SL & -.20 & .40 & .20 & -.03 & .01 & .33 & .34 \\
\hline $\mathbf{R M}$ & -.28 & .42 & .20 & -.03 & -.01 & .35 & .39 \\
\hline $\mathrm{CC}$ & -.22 & .31 & .19 & -.05 & -.02 & .31 & .34 \\
\hline HR & .06 & .18 & .12 & .15 & .14 & .14 & .12 \\
\hline SC & -.50 & .18 & .23 & -.35 & -.28 & .30 & .27 \\
\hline SM & -.12 & .00 & .30 & -.09 & -.02 & .21 & .10 \\
\hline $\mathbf{R D}$ & .35 & -.01 & -.09 & .37 & .36 & -.05 & -.04 \\
\hline $\mathbf{R E}$ & .35 & .11 & -.16 & .52 & .49 & -.10 & .10 \\
\hline RT & .51 & .09 & -.25 & .66 & .61 & -.11 & .05 \\
\hline
\end{tabular}

Note: $\mathbf{C R}=$ Consciousness Raising, $\mathbf{E R}=$ Environmental Reevaluation, $\mathbf{D R}=$ Dramatic Relief, $\mathbf{S R}=$ Self Reevaluation, $\mathbf{S L}=$ Self Liberation, $\mathbf{R M}=$ Reinforcement Management , $\mathrm{CC}=$ Counter Conditioning, $\mathbf{H R}=$ Helping Relationship, $\mathrm{SC}=$ Stimulus Control, $\mathbf{S M}=$ Self Monitoring, $\mathbf{R D}=$ Repression/Denial, $\mathbf{R E}=$ Reactance, $\mathbf{R T}=$ Rationalization

Correlations $\geq .11$ in magnitude are significant at $\mathrm{Q}<.01$

Correlations $\geq .20$ in magnitude are in bold 
Table 6-11: Means, standard deviations, and ANOVA results for Processes by the Stages of Cessation

\begin{tabular}{|c|c|c|c|c|c|c|}
\hline & $\begin{array}{c}\mathbf{P C} \\
(223) \\
\end{array}$ & $\begin{array}{l}\text { CONT } \\
(59) \\
\end{array}$ & $\begin{array}{c}\text { PREP } \\
(21) \\
\end{array}$ & $\begin{array}{c}\text { AC'TION } \\
(43) \\
\end{array}$ & $\begin{array}{c}\text { MAIN } \\
(80) \\
\end{array}$ & $\stackrel{F}{\omega^{2}}{ }^{2}$ \\
\hline \multirow[t]{2}{*}{$\mathrm{CR}$} & 2.36 & 2.55 & 2.58 & 2.46 & 2.74 & $4.02 *$ \\
\hline & $(.66)$ & $(.60)$ & $(.70)$ & $(.84)$ & $(1.01)$ & $\mathrm{PC}<\mathrm{M}$ \\
\hline \multirow[t]{2}{*}{ ER } & 2.58 & 2.60 & 2.64 & 2.79 & 3.13 & $5.59 *$ \\
\hline & (.83) & $(.82)$ & $(.90)$ & (.93) & $(1.12)$ & $\mathrm{PC}, \mathrm{C}<\mathrm{M}$ \\
\hline \multirow[t]{2}{*}{ DR } & 2.57 & 2.75 & 2.98 & 2.60 & 2.92 & $3.17^{*}$ \\
\hline & (.84) & $(.79)$ & $(.71)$ & $(.83)$ & $(1.04)$ & $\mathrm{PC}<\mathrm{M}$ \\
\hline \multirow[t]{2}{*}{ SR } & 1.99 & 2.36 & 2.76 & 1.83 & 1.82 & $9.55^{*}$ \\
\hline & $(.74)$ & (.85) & $(.92)$ & $(.59)$ & $(.86)$ & $\mathrm{PC}<\mathrm{C}, \mathrm{P} ; \mathrm{C}, \mathrm{P}>\mathrm{A}, \mathrm{M}$ \\
\hline \multirow[t]{2}{*}{ SL } & 2.56 & 2.78 & 3.18 & 2.72 & 2.71 & $2.69^{*}$ \\
\hline & $(.74)$ & $(.69)$ & (.99) & (.94) & $(1.24)$ & $\mathrm{PC}<\mathrm{P}$ \\
\hline \multirow[t]{2}{*}{$\mathrm{RM}$} & 2.13 & 2.33 & 2.48 & 2.32 & 2.45 & $3.37 *$ \\
\hline & $(.68)$ & $(.66)$ & $(.83)$ & $(.75)$ & $(1.03)$ & $\mathrm{PC}<\mathrm{M}$ \\
\hline \multirow[t]{2}{*}{$\mathrm{CC}$} & 2.21 & 2.45 & 2.57 & 2.45 & 2.51 & $2.80^{*}$ \\
\hline & $(.73)$ & $(.77)$ & (.87) & $(.89)$ & $(1.18)$ & $\mathrm{PC}<\mathrm{M}($ at $\mathrm{p}<.10)$ \\
\hline \multirow[t]{2}{*}{$\mathrm{HR}$} & 3.38 & 3.17 & 3.68 & 3.29 & 3.16 & \multirow[t]{2}{*}{1.16} \\
\hline & $(1.20)$ & $(1.07)$ & $(1.17)$ & $(1.15)$ & $(1.38)$ & \\
\hline \multirow[t]{2}{*}{$\mathrm{SC}$} & 2.16 & 2.36 & 2.50 & 2.44 & 2.97 & $15.05^{*}$ \\
\hline & $(.65)$ & $(.70)$ & $(.66)$ & $(.88)$ & $(1.13)$ & $\mathrm{PC}, \mathrm{C}, \mathrm{A}<\mathrm{M}$ \\
\hline \multirow[t]{2}{*}{$\mathrm{SM}$} & 3.28 & 3.32 & 3.30 & 3.37 & 3.69 & $3.74^{*}$ \\
\hline & $(.77)$ & $(.72)$ & $(.93)$ & (.81) & $(.93)$ & $\mathrm{PC}<\mathrm{M}$ \\
\hline \multirow[t]{2}{*}{$\mathrm{RD}$} & 2.78 & 2.67 & 2.42 & 2.55 & 2.14 & $9.95^{*}$ \\
\hline & $(.78)$ & $(.69)$ & $(.72)$ & (.83) & $(.86)$ & $\mathrm{PC}, \mathrm{C}, \mathrm{A}>\mathrm{M}$ \\
\hline \multirow[t]{2}{*}{ RE } & 2.40 & 2.47 & 2.38 & 2.11 & 1.88 & $7.00^{*}$ \\
\hline & $(.85)$ & $(.81)$ & $(.85)$ & $(.82)$ & $(.76)$ & $\mathrm{PC}, \mathrm{C}>\mathrm{M}$ \\
\hline \multirow[t]{2}{*}{ RT } & 3.07 & 3.00 & 2.98 & 2.61 & 1.96 & $41.33^{*}$ \\
\hline & $(.68)$ & (.62) & $(.70)$ & (.79) & (.64) & $\mathrm{PC}, \mathrm{C}>\mathrm{A}, \mathrm{M} ; \mathrm{P}, \mathrm{A}>\mathrm{M}$ \\
\hline
\end{tabular}

Note: * F-test significant at $\mathrm{p}<.05$

Pair-wise difference by Tukey tests, $\mathrm{p}<.05$

Degrees of freedom for F-test vary from $(4,428)$ to $(4,434)$ 
Table 6-12: Means, standard deviations, and ANOVA results for Processes by the Stages with intentional criteria for Action and Maintenance

\begin{tabular}{|c|c|c|c|c|c|c|}
\hline & $\begin{array}{r}\mathbf{P C} \\
(223) \\
\end{array}$ & $\begin{array}{c}\text { CONT } \\
(59) \\
\end{array}$ & $\begin{array}{c}\text { PREP } \\
(2 I) \\
\end{array}$ & $\begin{array}{c}\text { ACTION } \\
(28) \\
\end{array}$ & $\begin{array}{c}\text { MAIN } \\
(73) \\
\end{array}$ & $\underline{\mathbf{F}} \omega_{\text {Tukey results }}^{\omega^{2}}$ \\
\hline \multirow[t]{2}{*}{$\mathrm{CR}$} & 2.36 & 2.55 & 2.58 & 2.74 & 2.79 & $5.54^{*}$ \\
\hline & $(.66)$ & $(.60)$ & $(.70)$ & $(.87)$ & $(1.03)$ & $\mathrm{PC}<\mathrm{M}$ \\
\hline \multirow[t]{2}{*}{ ER } & 2.58 & 2.60 & 2.64 & 3.06 & 3.23 & $8.36^{*}$ \\
\hline & $(.83)$ & $(.82)$ & $(.90)$ & (.97) & $(1.09)$ & $\mathrm{PC}, \mathrm{C}<\mathrm{M}$ \\
\hline \multirow[t]{2}{*}{ DR } & 2.57 & 2.75 & 2.98 & 2.81 & 2.95 & $3.38^{*}$ \\
\hline & $(.84)$ & $(.79)$ & $(.71)$ & $(.90)$ & $(1.05)$ & $\mathrm{PC}<\mathrm{M}$ \\
\hline \multirow[t]{2}{*}{ SR } & 1.99 & 2.36 & 2.76 & 1.99 & 1.85 & $8.02^{*}$ \\
\hline & $(.74)$ & $(.85)$ & $(.92)$ & (.64) & $(.85)$ & $\mathrm{PC}, \mathrm{M}<\mathrm{C} ; \mathrm{PC}, \mathrm{A}, \mathrm{M}<\mathrm{P}$ \\
\hline \multirow[t]{2}{*}{ SL } & 2.56 & 2.78 & 3.18 & 2.95 & 2.78 & $3.60^{*}$ \\
\hline & $(.74)$ & $(.69)$ & $(.99)$ & $(.97)$ & $(1.24)$ & $\mathrm{PC}<\mathrm{P}$ \\
\hline \multirow[t]{2}{*}{$\mathrm{RM}$} & 2.13 & 2.33 & 2.48 & 2.53 & 2.53 & $5.11^{*}$ \\
\hline & $(.68)$ & (.66) & $(.83)$ & $(.84)$ & $(1.04)$ & $\mathrm{PC}<\mathrm{M}$ \\
\hline \multirow[t]{2}{*}{$\mathrm{CC}$} & 2.21 & 2.45 & 2.57 & 2.53 & 2.57 & $3.45^{*}$ \\
\hline & $(.73)$ & $(.77)$ & $(.87)$ & $(.94)$ & $(1.19)$ & $\mathrm{PC}<\mathrm{M}$ \\
\hline \multirow[t]{2}{*}{ HR } & 3.38 & 3.17 & 3.68 & 3.33 & 3.12 & \multirow[t]{2}{*}{1.32} \\
\hline & $(1.20)$ & $(1.07)$ & $(1.17)$ & $(1.16)$ & $(1.39)$ & \\
\hline \multirow[t]{2}{*}{$\mathrm{SC}$} & 2.16 & 2.36 & 2.50 & 2.70 & 3.02 & $17.38 *$ \\
\hline & $(.65)$ & $(.70)$ & $(.66)$ & $(.96)$ & $(1.10)$ & $\mathrm{PC}<\mathrm{A}, \mathrm{M} ; \mathrm{C}<\mathrm{M}$ \\
\hline \multirow[t]{2}{*}{$S M$} & 3.28 & 3.32 & 3.30 & 3.54 & 3.72 & $4.31^{*}$ \\
\hline & $(.77)$ & $(.72)$ & $(.93)$ & $(.90)$ & (.94) & $\mathrm{PC}, \mathrm{C}<\mathrm{M}$ \\
\hline \multirow[t]{2}{*}{ RD } & 2.78 & 2.67 & 2.42 & 2.37 & 2.12 & $10.22^{*}$ \\
\hline & $(.78)$ & $(.69)$ & $(.72)$ & $(.83)$ & $(.87)$ & $\mathrm{PC}, \mathrm{C}>\mathrm{M}$ \\
\hline \multirow[t]{2}{*}{$\mathrm{RE}$} & 2.40 & 2.47 & 2.38 & 1.92 & 1.88 & $7.50^{*}$ \\
\hline & $(.85)$ & $(.81)$ & $(.85)$ & $(.62)$ & $(.78)$ & $\mathrm{PC}, \mathrm{C}>\mathrm{A}, \mathrm{M}$ \\
\hline \multirow[t]{2}{*}{ RT } & 3.07 & 3.00 & 2.98 & 2.32 & 1.92 & $46.27^{*}$ \\
\hline & $(.68)$ & $(.62)$ & $(.70)$ & $(.67)$ & $(.62)$ & $\mathrm{PC}, \mathrm{C}, \mathrm{P}>\mathrm{A}, \mathrm{M}$ \\
\hline
\end{tabular}

Note: * F-test significant at $\mathrm{p}<.05$

Pair-wise difference by Tukey tests, $\mathrm{p}<.05$

Degrees of freedom for F-test vary from $(4,390)$ to $(4,395)$ 


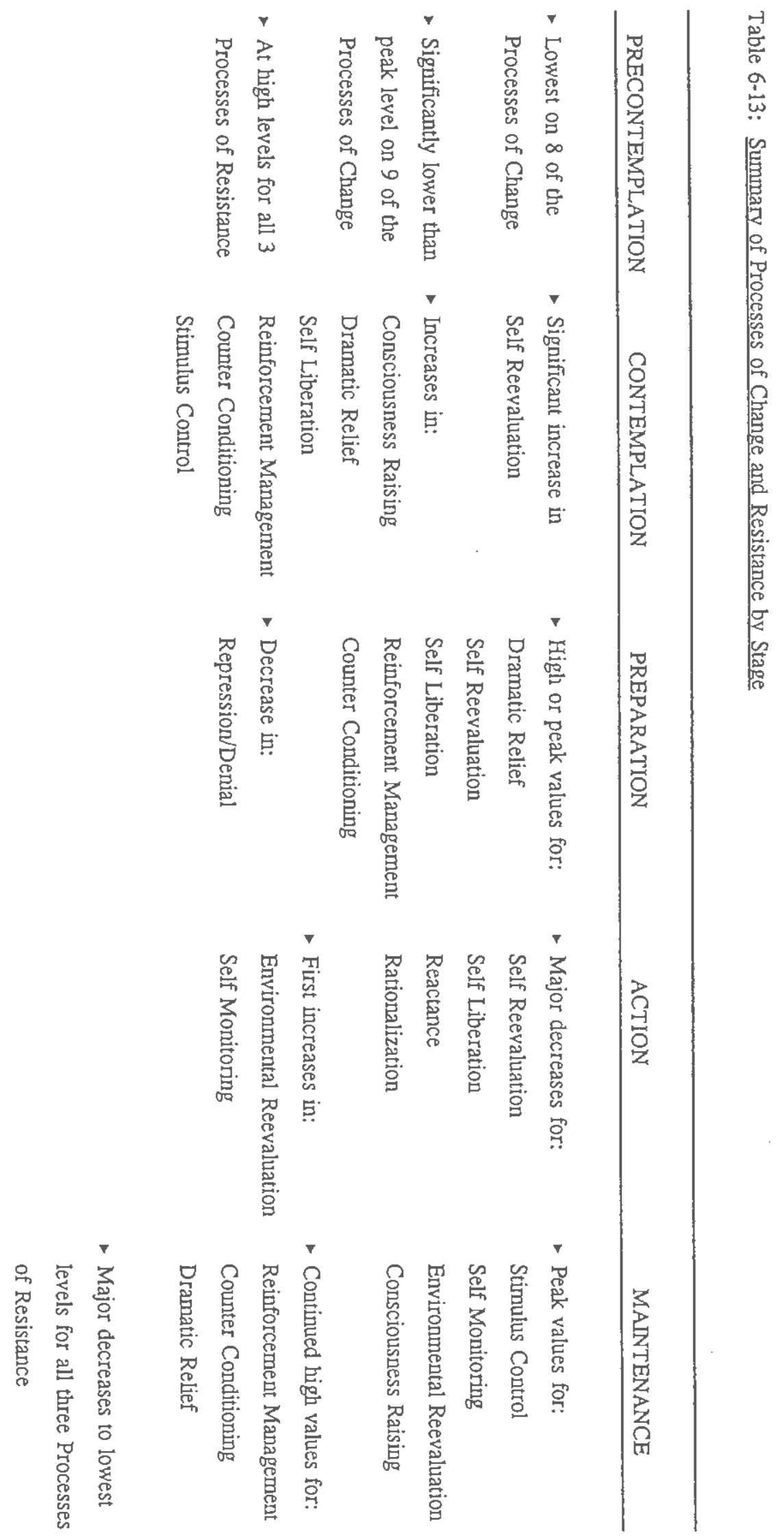


Table 6-14: Means, standard deviations, and ANOVA results for Processes by gender

\begin{tabular}{|c|c|c|c|c|}
\hline & FEMALES & MALES & $\underline{\mathbf{F}}$ & $\omega^{2}$ \\
\hline \multirow[t]{2}{*}{$\mathrm{CR}$} & 2.48 & 2.48 & 0.00 & \\
\hline & $(.75)$ & $(.78)$ & & \\
\hline \multirow[t]{2}{*}{$\mathrm{ER}$} & 2.75 & 2.65 & 1.45 & \\
\hline & $(.89)$ & $(.97)$ & & \\
\hline \multirow[t]{2}{*}{$\mathrm{DR}$} & 2.82 & 2.44 & $19.09^{*}$ & .033 \\
\hline & $(.88)$ & $(.83)$ & & \\
\hline \multirow[t]{2}{*}{$\mathrm{SR}$} & 1.92 & 2.22 & $15.47^{*}$ & .026 \\
\hline & $(.78)$ & $(.82)$ & & \\
\hline \multirow[t]{2}{*}{$\mathrm{SL}$} & 2.62 & 2.74 & 1.56 & \\
\hline & $(.94)$ & $(.78)$ & & \\
\hline \multirow[t]{2}{*}{$\mathrm{RM}$} & 2.19 & 2.37 & $6.28 *$ & .005 \\
\hline & $(.76)$ & $(.79)$ & & \\
\hline \multirow[t]{2}{*}{$\mathrm{CC}$} & 2.25 & 2.49 & $8.73^{*}$ & .011 \\
\hline & $(.86)$ & (.84) & & \\
\hline \multirow[t]{2}{*}{$\mathrm{HR}$} & 3.32 & 3.30 & .03 & \\
\hline & $(1.27)$ & $(1.12)$ & & \\
\hline \multirow[t]{2}{*}{$S C$} & 2.42 & 2.32 & 1.55 & \\
\hline & $(.89)$ & $(.75)$ & & \\
\hline \multirow[t]{2}{*}{ SM } & 3.50 & 3.15 & $18.50^{*}$ & .032 \\
\hline & $(.81)$ & $(.79)$ & & \\
\hline \multirow[t]{2}{*}{$\mathrm{RD}$} & 2.55 & 2.70 & 3.40 & \\
\hline & $(.85)$ & $(.77)$ & & \\
\hline \multirow[t]{2}{*}{ RE } & 2.15 & 2.52 & $20.56^{*}$ & .037 \\
\hline & $(.84)$ & $(.82)$ & & \\
\hline \multirow[t]{2}{*}{ RT } & 2.76 & 2.86 & 2.58 & \\
\hline & $(.81)$ & $(.77)$ & & \\
\hline
\end{tabular}

Note: * F-test significant at $\mathrm{p}<.05$

Degrees of freedom for F-test vary from $(1,428)$ to $(1,434)$ 
Figure 6-1: Item loadings for the 10 Processes of the Correlated Structural Model

I recall information about the benefits of not drinking a lot

I think about information I have heard about problems associated with drinking too much

I look for more information about the effects of alcohol

I pay attention to articles about the health effects of alcohol

I ask other students about their opinions about drinking

I recall information people have given me on the benefits of drinking less
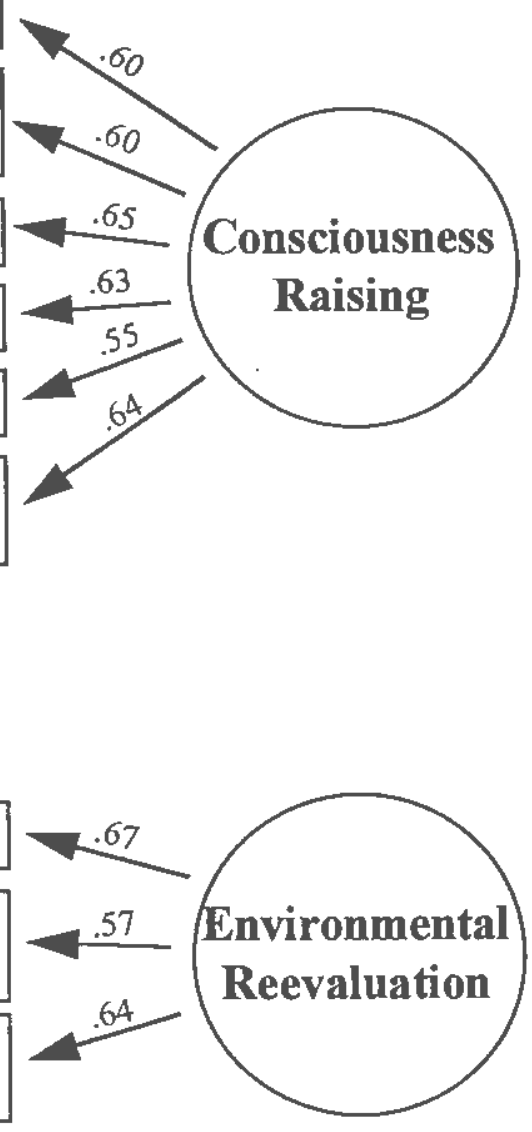

I wonder how much less society's health care cost would be if noone drank a lot

I wonder about all the ways excessive drinking affects society

I consider the idea that if people drank less the world would be a better place

I get upset when I think about the problems drinking causes

I am emotionally moved when I hear of the harm that excessive drinking has caused

Hearing about research on the effects of alcohol use worries me

Dramatic portrayals of the evils of drinking affect me emotionally

I react emotionally to warnings about excessive drinking

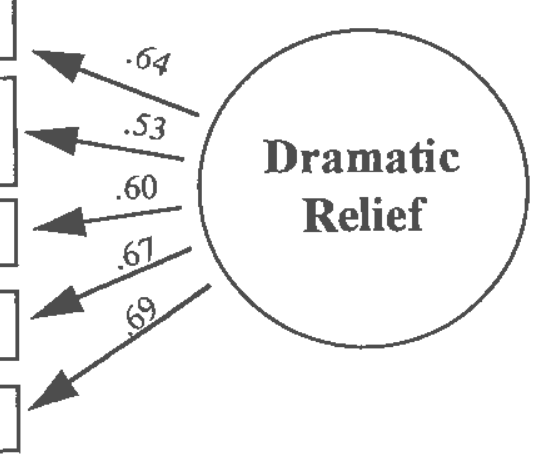


Figure 6-1(cont): Item loadings for the 10 Processes of the Correlated Structural Model

I feel that being content with myself means changing my drinking habits

I struggle with the fact that my drinking habits contradict my changing view of myself

The way I drink makes me disappointed in myself

I wonder about my drinking habits

I stop and think that my drinking is causing problems for others

I make commitments to myself to drink less

I use will power to control my drinking

I tell myself that I will drink less or not at all today

I remind myself that I am able to reduce the amount I drink

Other people treat me better when I do not drink too much

I do something nice for myself for making efforts to drink less

I punish myself if $I$ drink too much

I pat myself on the back for limiting my drinking

Others around me reinforce my not drinking too much

When I am tempted to have another (or my first) drink I think about or do something else instead

I find that keeping busy helps me drink less

I calm myself down when I get the urge to drink
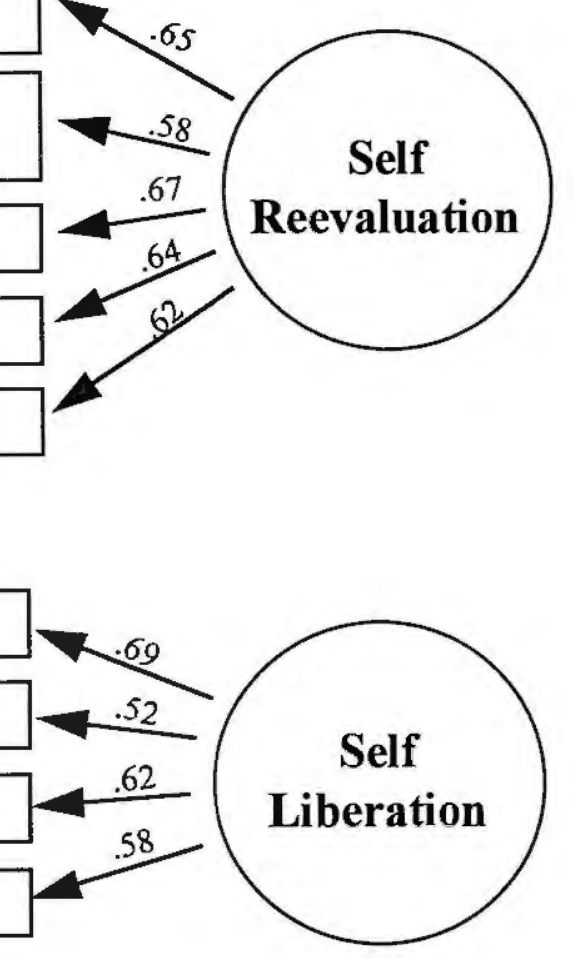
Figure 6-1(cont.): Item loadings for the 10 Processes of the Correlated Structural Model

\begin{tabular}{|l|}
\hline I am open with at least one person I can trust about my drinking \\
\hline I have someone who listens when I need to talk about my drinking \\
\hline $\begin{array}{c}\text { I have someone I can count on when I'm having problems with } \\
\text { my drinking }\end{array}$ \\
\hline
\end{tabular}

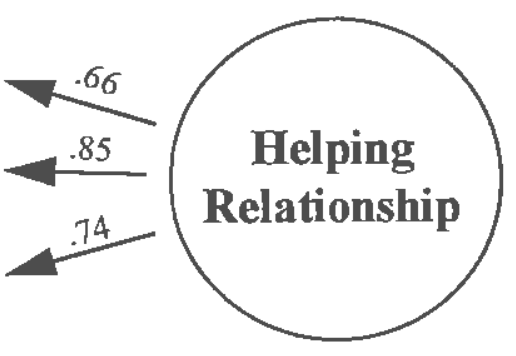

I avoid places where there will be a lot of drinking
I avoid places or events where I tend to drink too much

I avoid drinking with people who are heavy drinkers

I avoid those who tend to push drinks on me

I try to make friends with people who are not heavy drinkers

I seek out people who do things that do not involve a lot of drinking
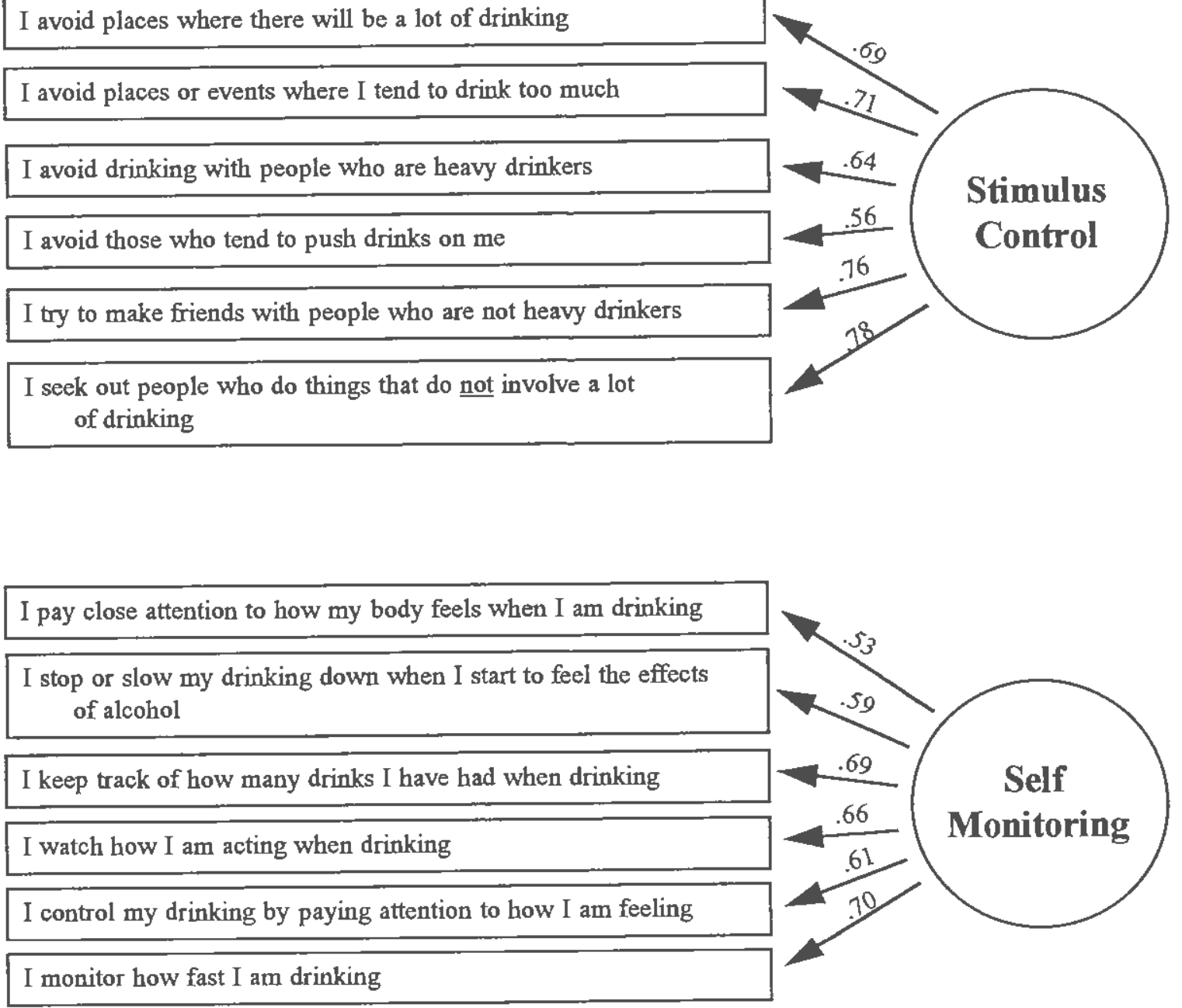
Figure 6-2: Factor loadings for the Two-Factor Hierarchical Structural Model

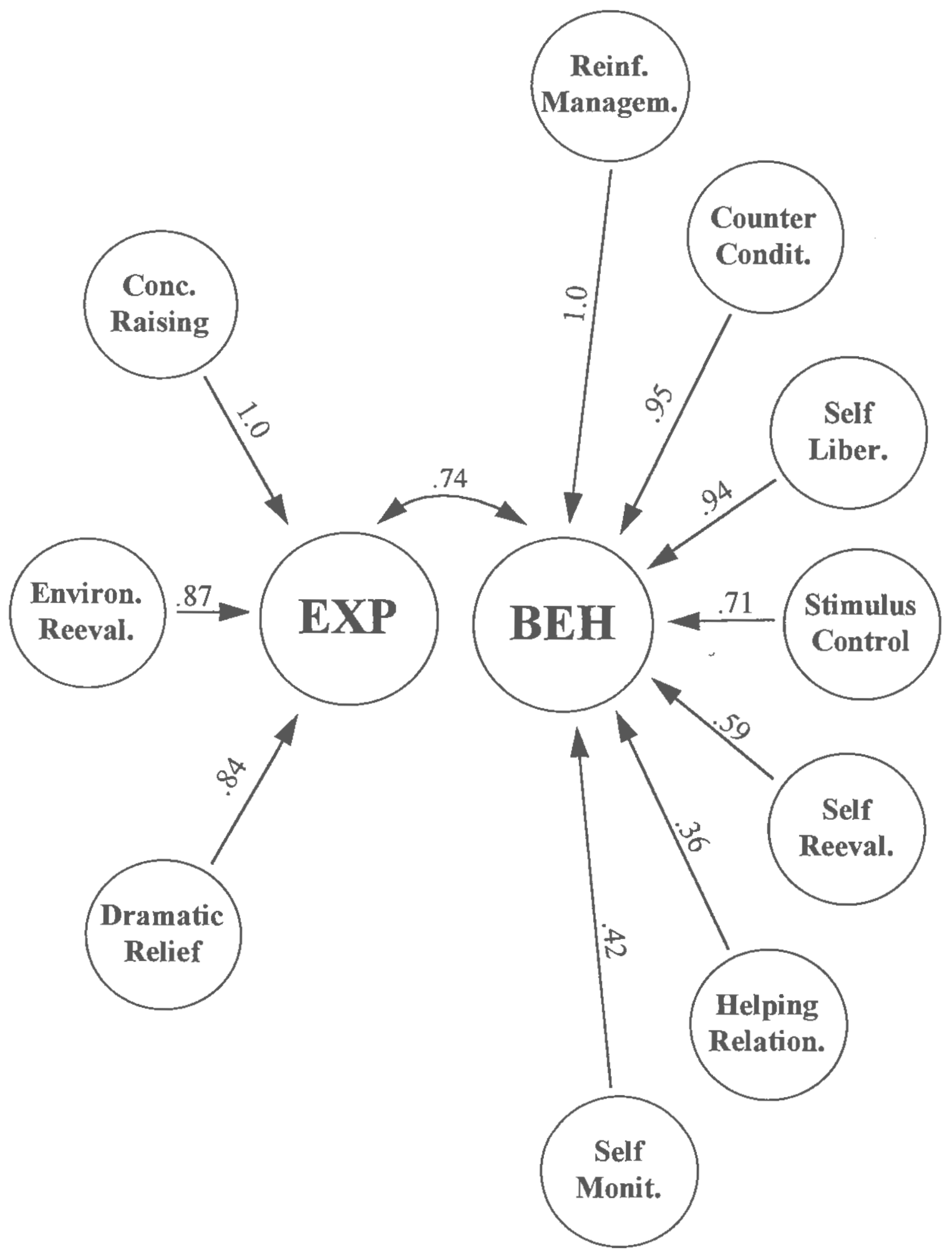


Figure 6-3: Structural model for three Processes of Resistance scales

\begin{tabular}{l}
$\begin{array}{l}\text { I think my drinking is OK because I will drink less after I } \\
\text { graduate from college }\end{array}$ \\
$\begin{array}{l}\text { I feel that after a hard week at school I deserve to have a good } \\
\text { time drinking }\end{array}$ \\
$\begin{array}{l}\text { I feel that I need the real break from studying or working that } \\
\text { drinking can give me }\end{array}$ \\
\hline I think it is natural to drink a lot when you are in college \\
\hline I think that drinking a lot is just part of growing up \\
\hline \\
\hline
\end{tabular}
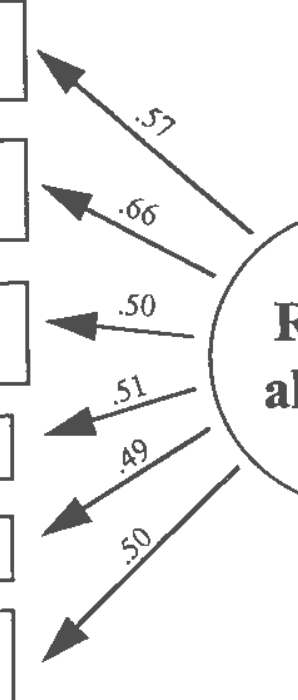

Rationalization

My anger at rules that restrict my right to drink makes me want to drink all the more

Rules saying that I can not drink make me want to drink all the more

Drinking as much as I want makes me feel like my own person

I get enjoyment out of getting away with illegal drinking

I think that it would be silly for me to not drink just because of a rule

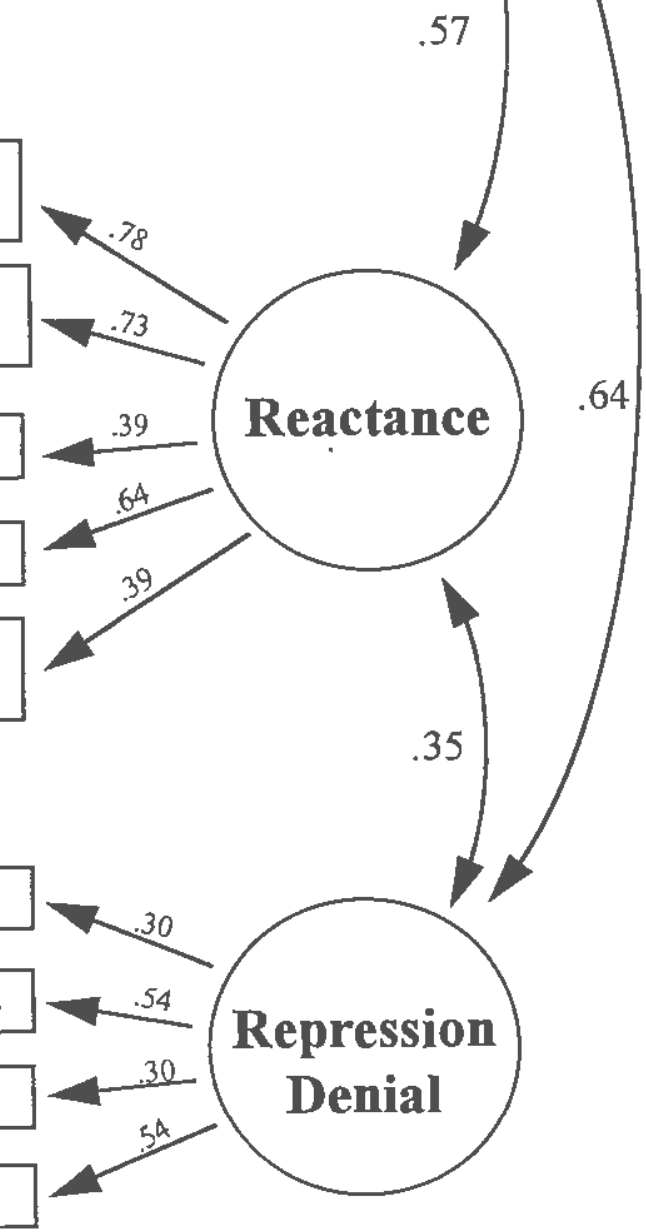


Figure 6-4: Structural model for two Processes of Resistance scales

\begin{tabular}{l}
$\begin{array}{l}\text { I think my drinking is OK because I will drink less after I } \\
\text { graduate from college }\end{array}$ \\
\begin{tabular}{|l|} 
I feel that after a hard week at school I deserve to have a good \\
time drinking
\end{tabular} \\
\hline $\begin{array}{l}\text { I feel that I need the real break from studying or working that } \\
\text { drinking can give me }\end{array}$ \\
\hline I think it is natural to drink a lot when you are in college \\
\hline I think that drinking a lot is just part of growing up \\
\hline I think that if I did not drink like most others my social life \\
would suffer
\end{tabular}
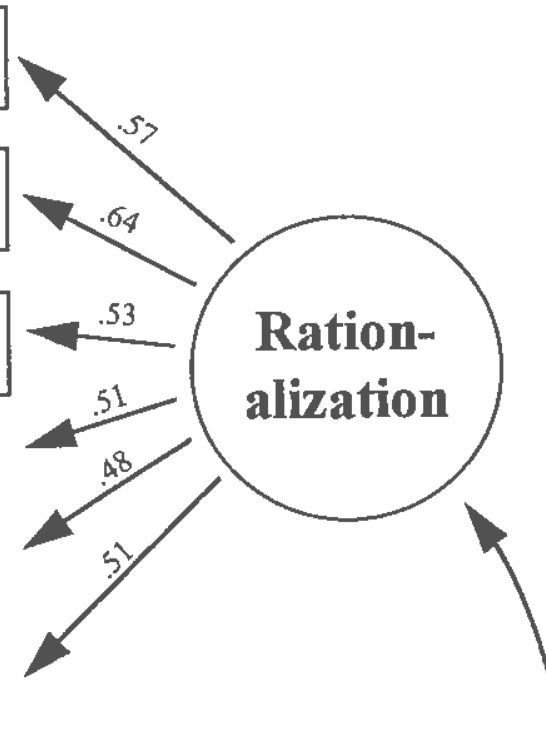

My anger at rules that restrict my right to drink makes me want to drink all the more

Rules saying that I can not drink make me want to drink all the more

Drinking as much as I want makes me feel like my own person

I get enjoyment out of getting away with illegal drinking

I think that it would be silly for me to not drink just because of a rule 
Figure 6-5: Correlated Hierarchical Model for the Processes of Change and Resistance

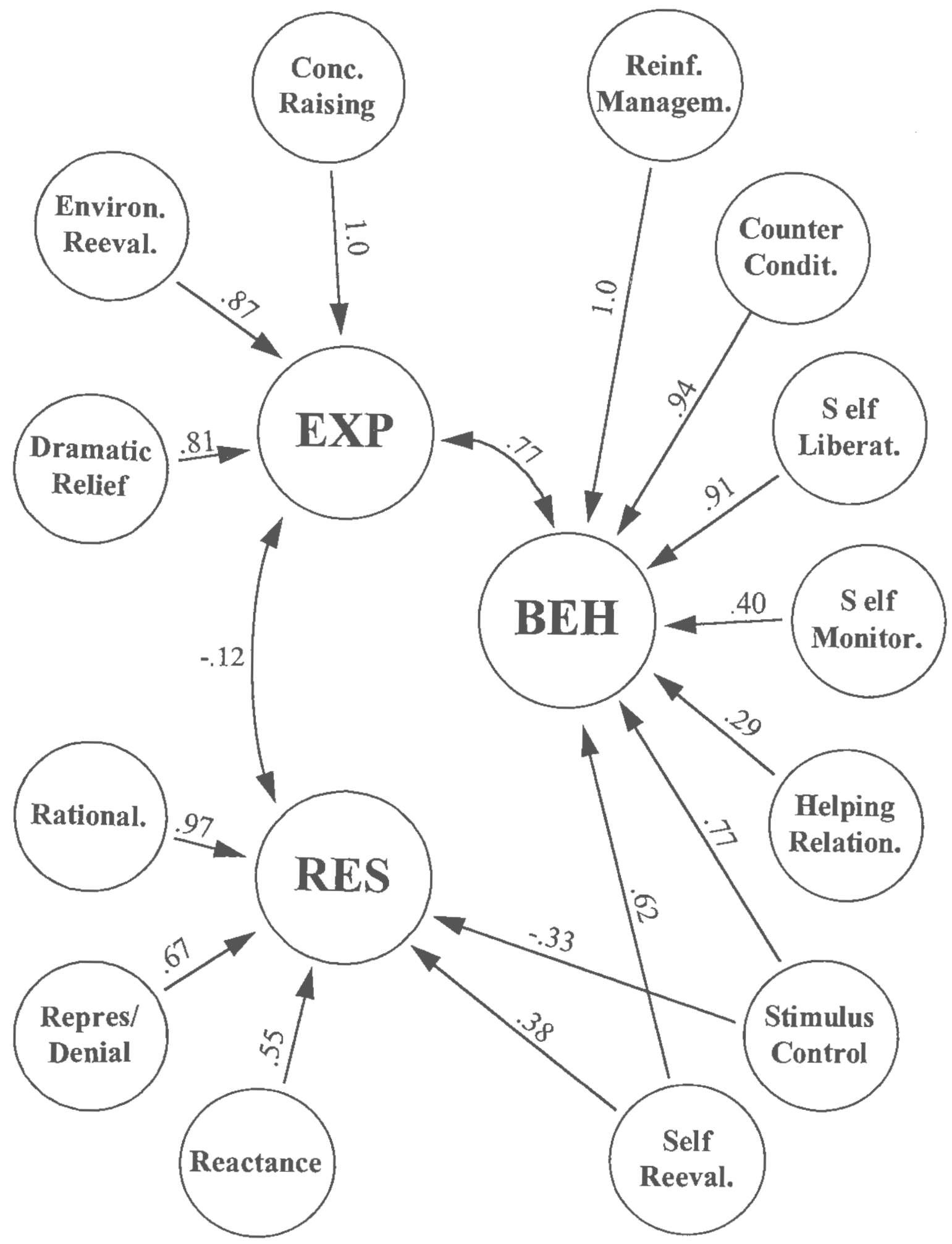


Figure 6-6: Third-order Hierarchical Model for the Processes of Change and Resistance

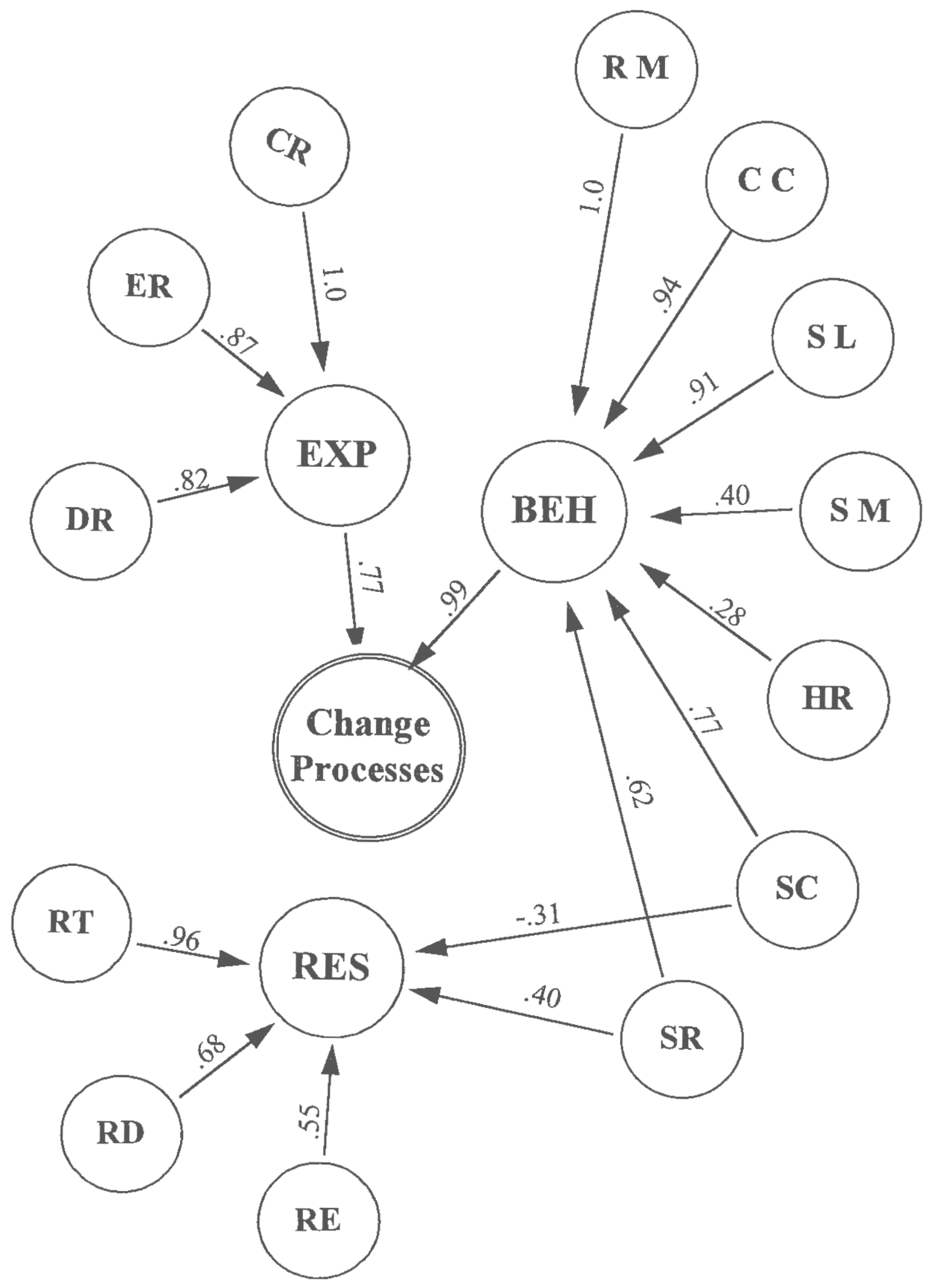


Figure 6-7 : Environmental Reevaluation and Consciousness Raising by Stage of Cessation

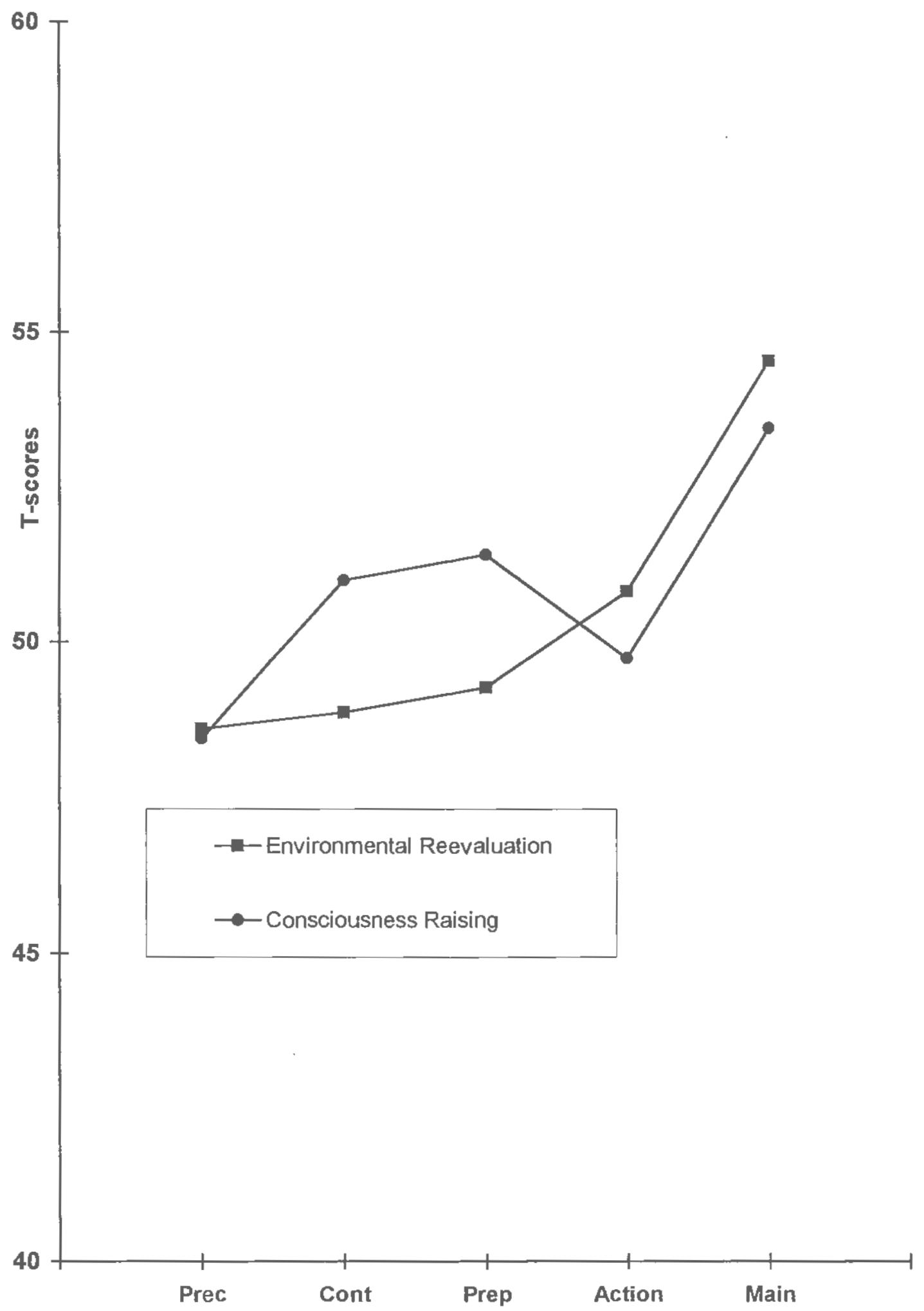


Figure 6-8: Dramatic Relief and Self Liberation by Stage of Cessation

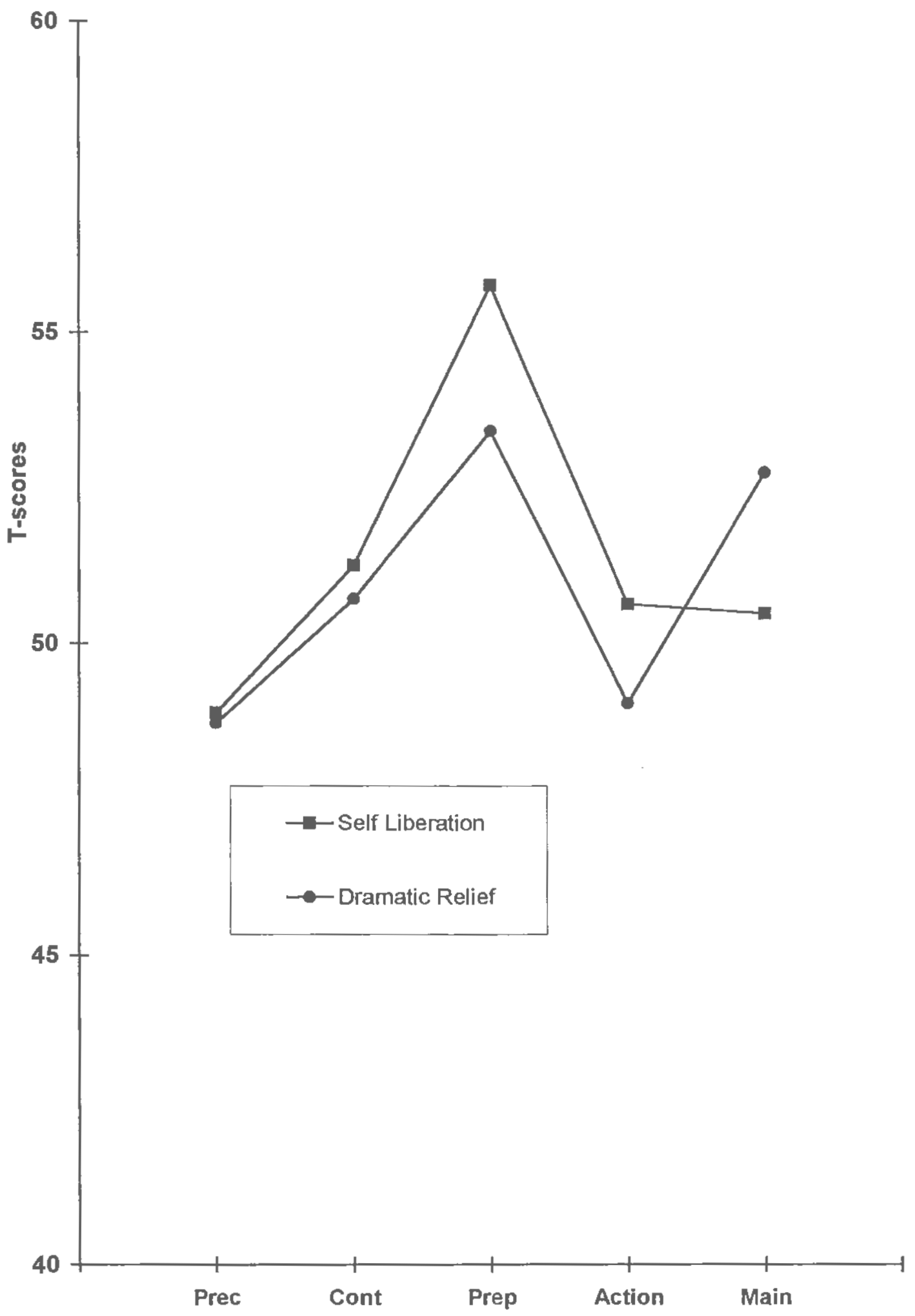


Figure 6-9: Stimulus Control and Self Reevaluation by Stage of Cessation

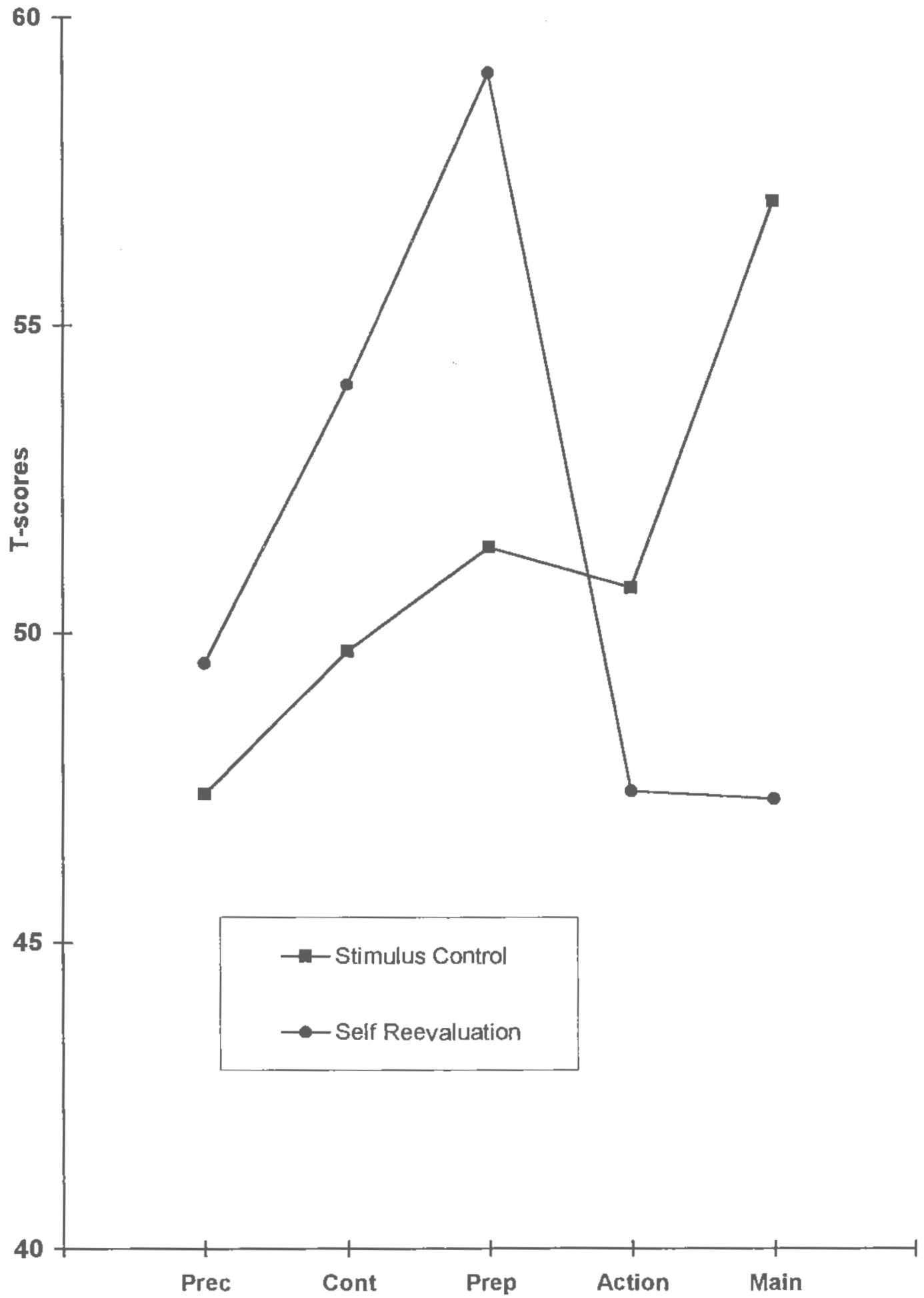


Figure 6-10: Reinforcement Management and Counter-Conditioning by Stage of Cessation

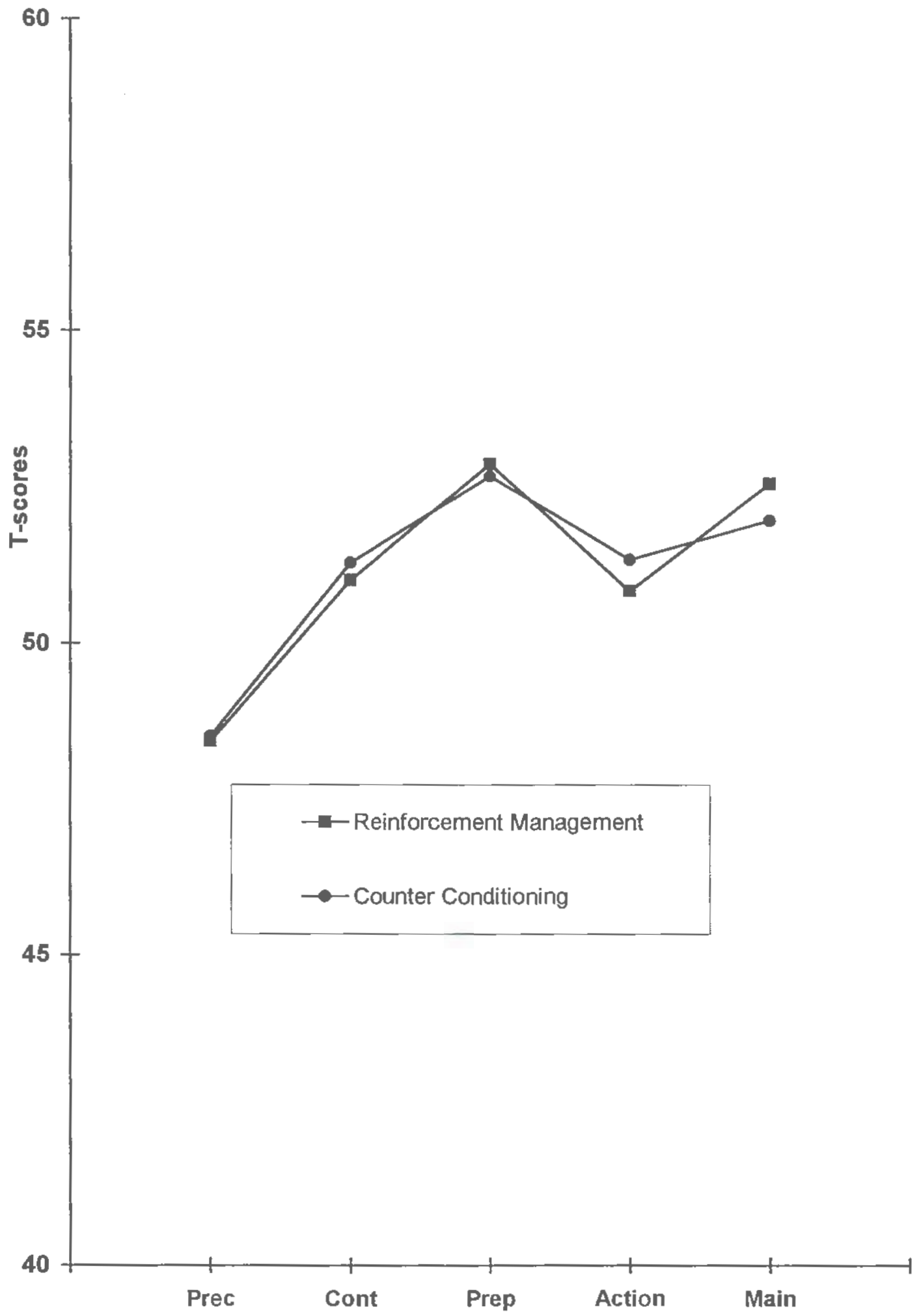


Figure 6-11: Helping Relationship and Self Management by Stage of Cessation

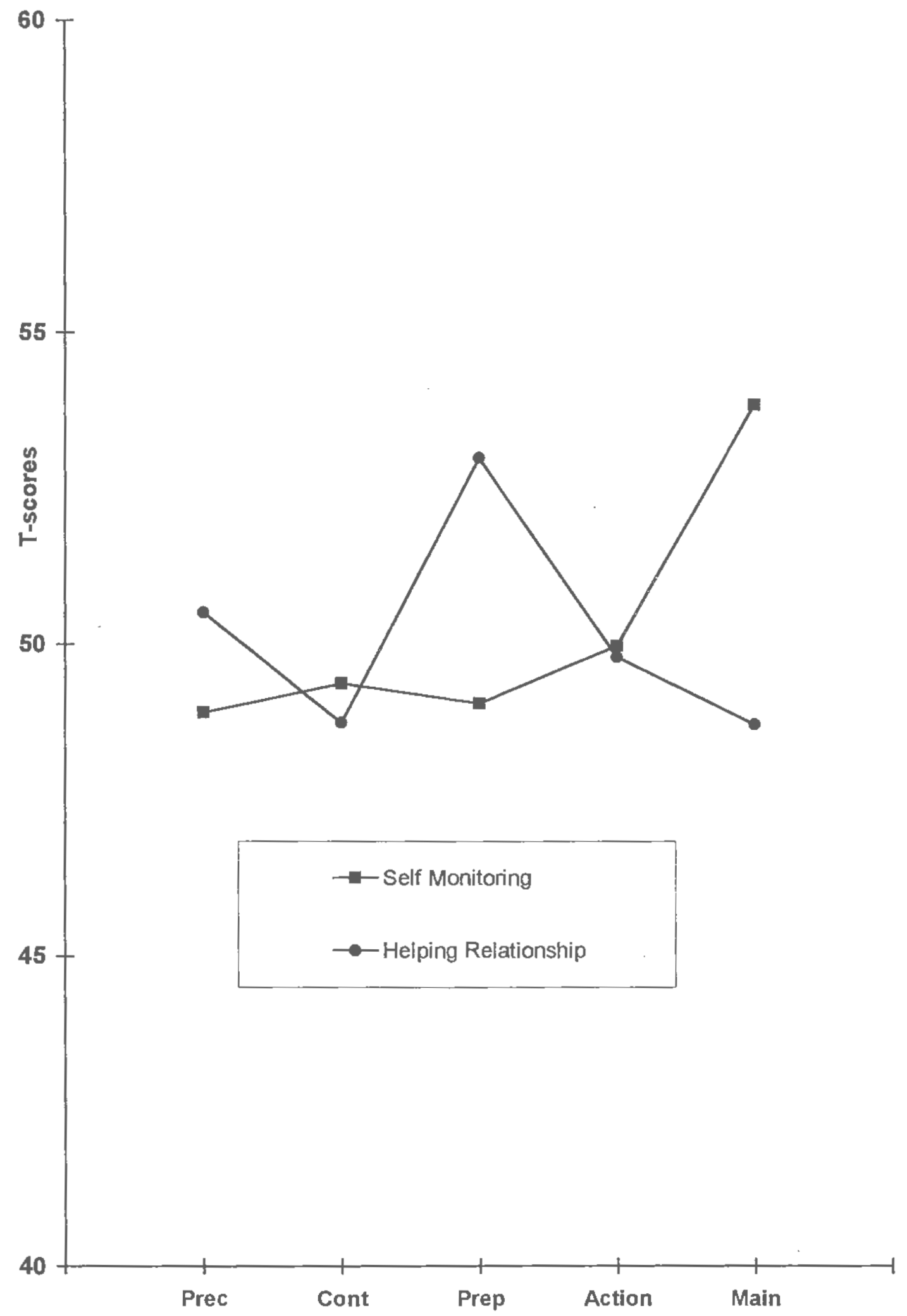


Figure 6-12: Repression/Denial, Reactance, and Rationalization by Stage of Cessation

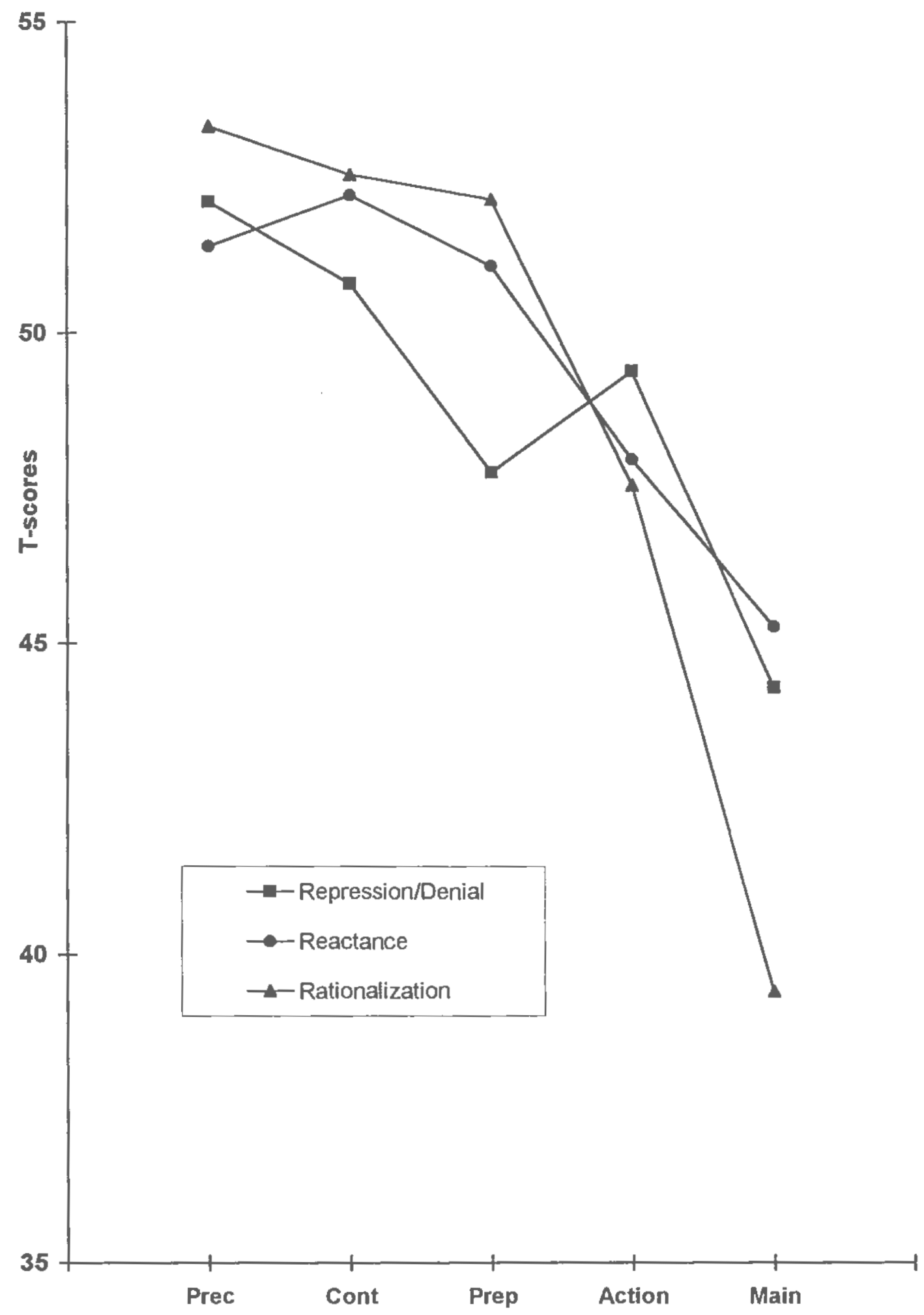


Figure 6-13 : Environmental Reevaluation and Consciousness Raising by Stage with intentional criterion for Action and Maintenance

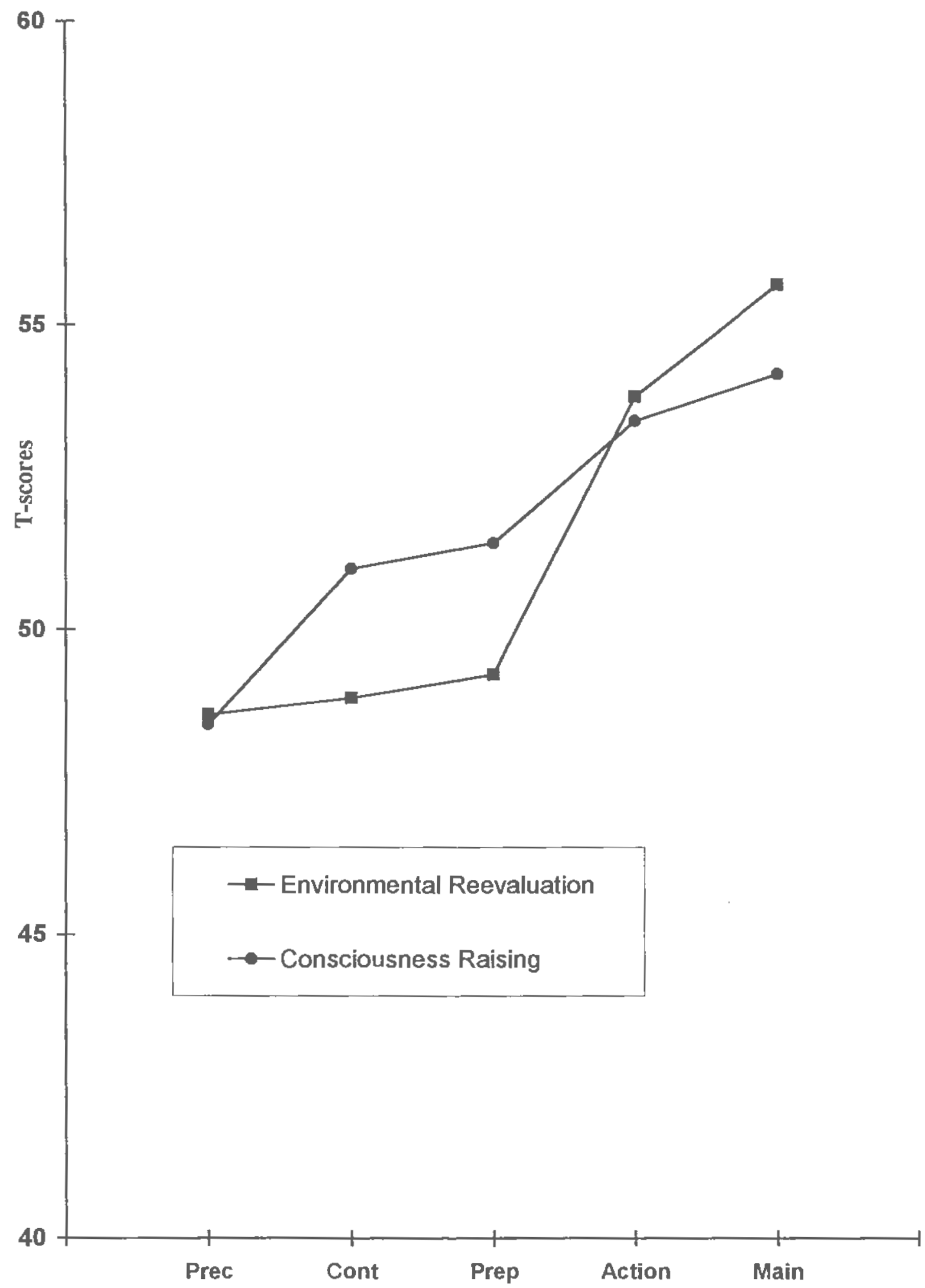


Figure 6-14: Dramatic Relief and Self Liberation by Stage with intentional criterion for Action and Maintenance

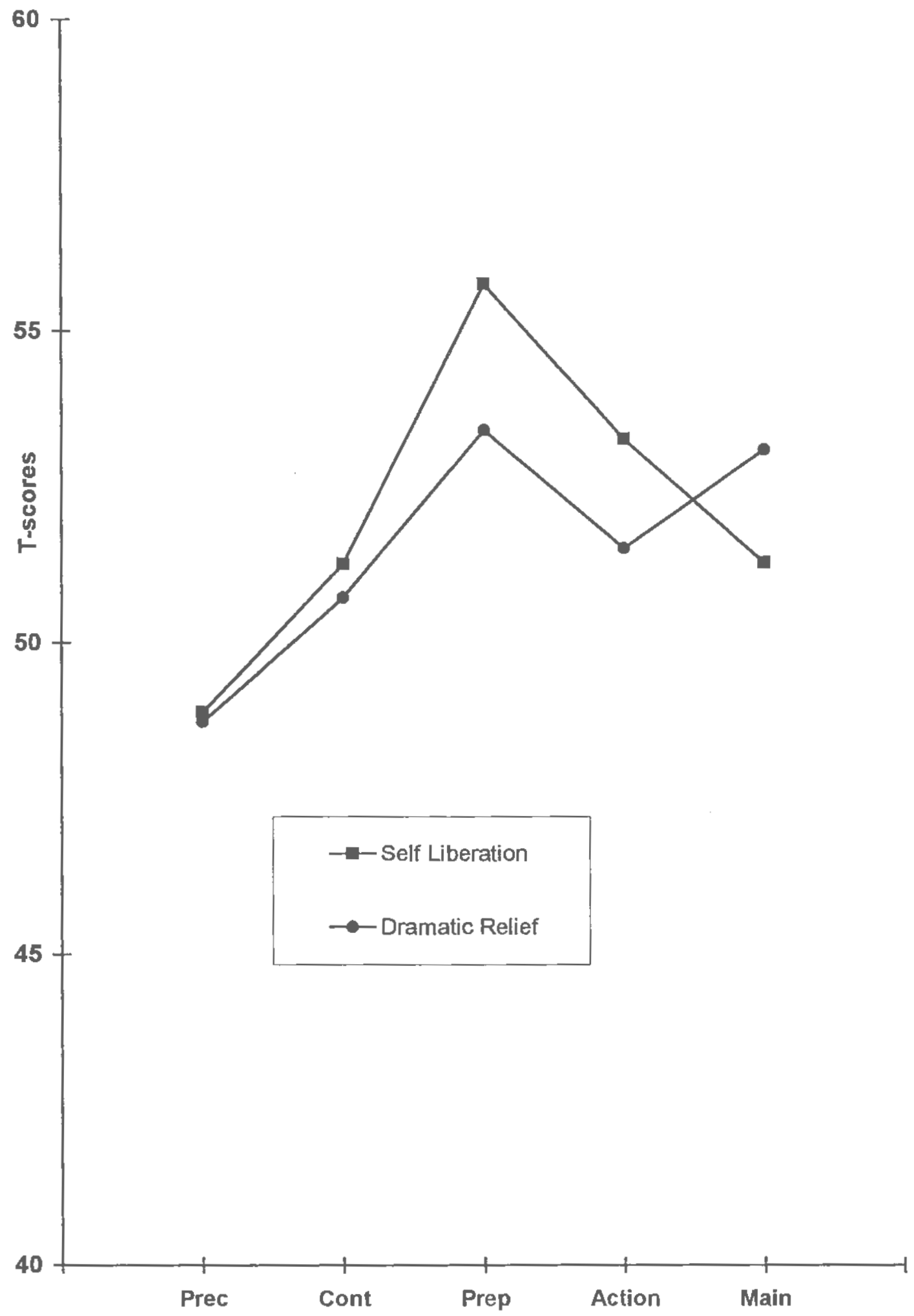


Figure 6-15: Stimulus Control and Self Reevaluation by Stage. with intentional criterion for Action and Maintenance

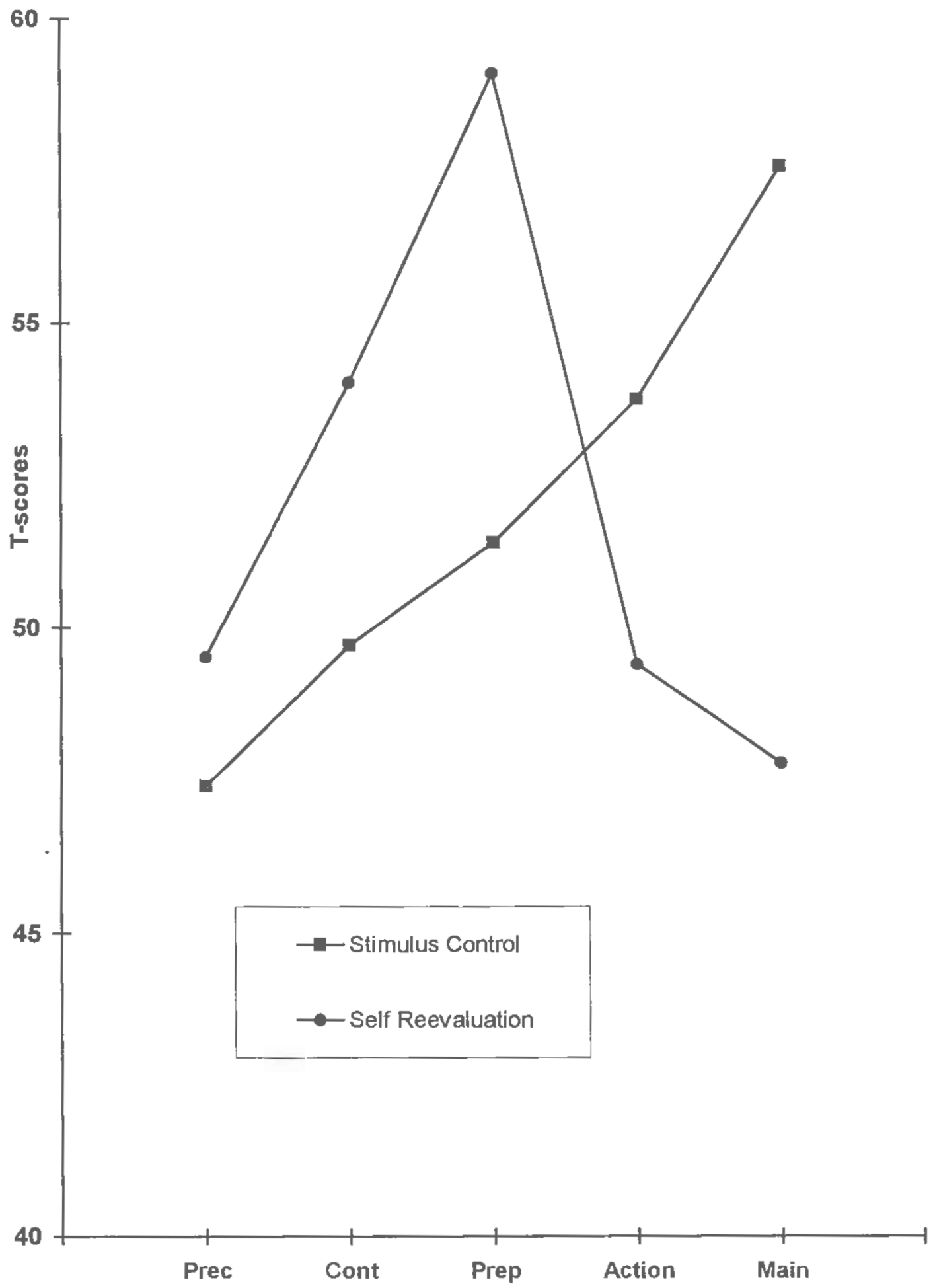


Figure 6-16: Reinforcement Management and Counter-Conditioning by Stage with intentional criterion for Action and Maintenance

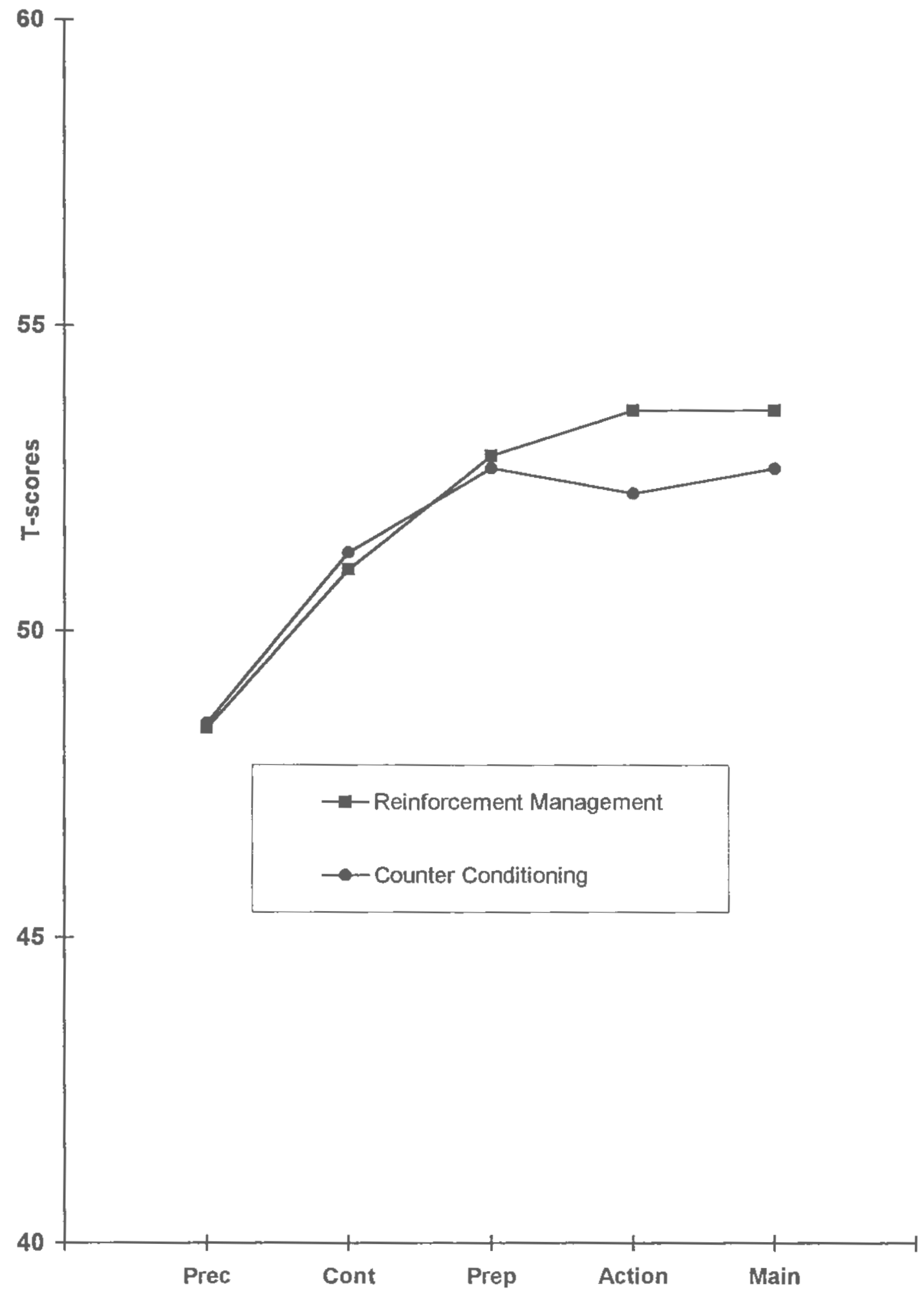


Figure 6-17 : Helping Relationship and Self Monitoring by Stage

with intentional criterion for Action and Maintenance

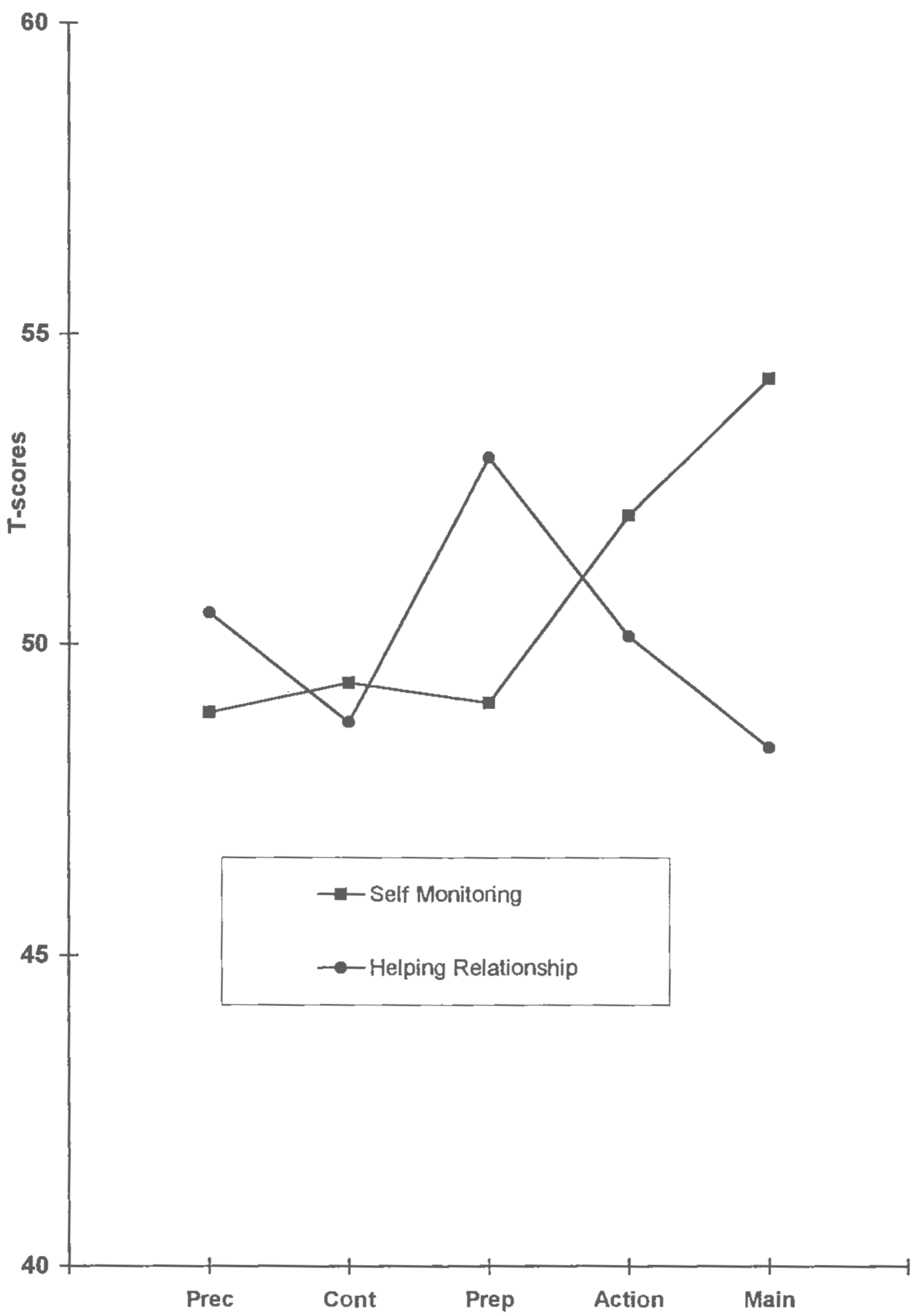


Figure 6-18: Repression/Denial, Reactance, and Rationalization by Stage with intentional criterion for Action and Maintenance

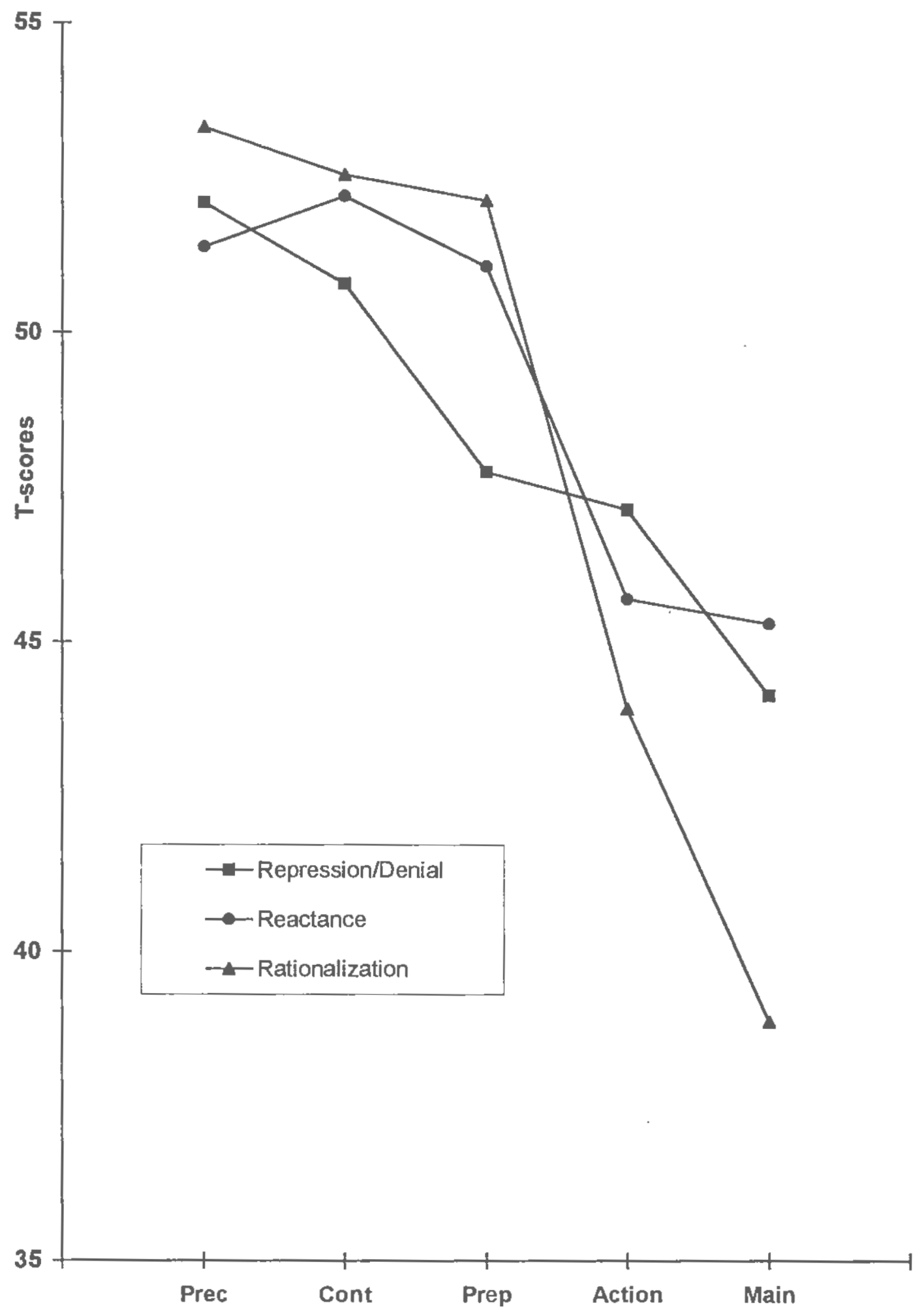


Figure 6-19: Reinforcement Management, Stimulus Control, Self Liberation, and Rationalization by Stage of Cessation

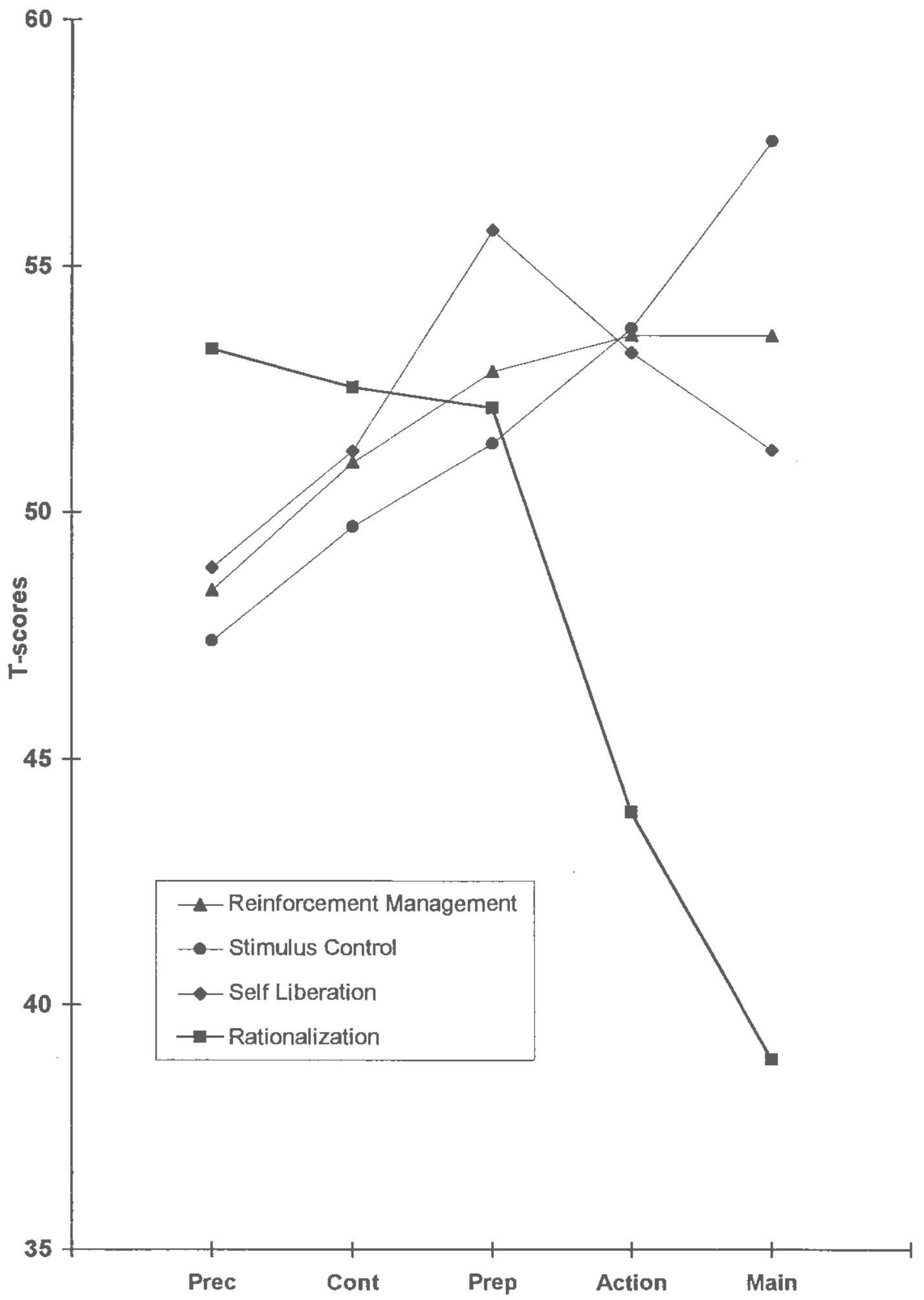


Figure 6-20: Environmental Reevaluation, Consciousness Raising, Self Reevalutaion, and Rationalization by Stage of Cessation

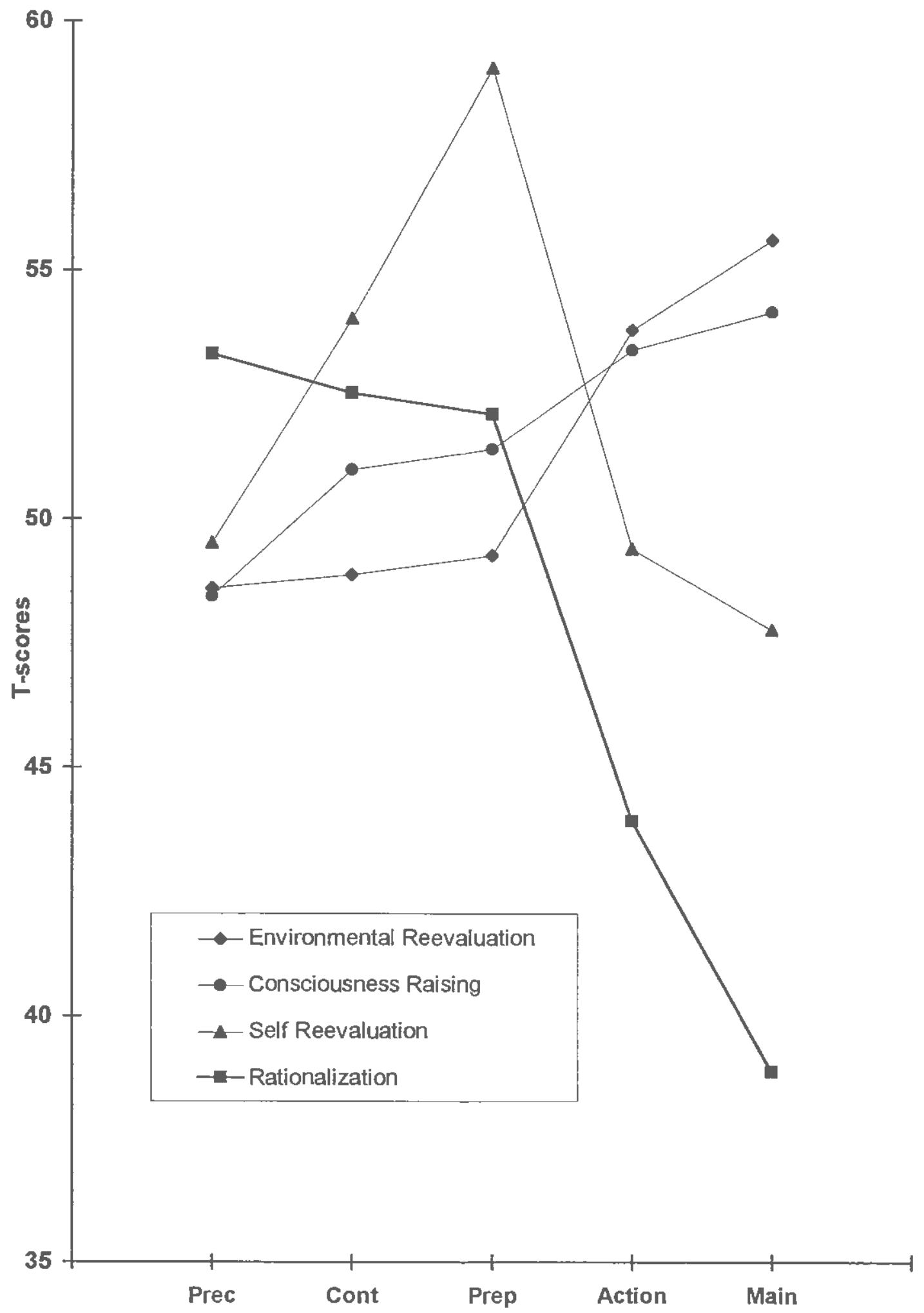


Figure 6-21: Self Monitoring, Counter Conditioning, Dramatic Relief, and

Rationalization by Stage of Cessation

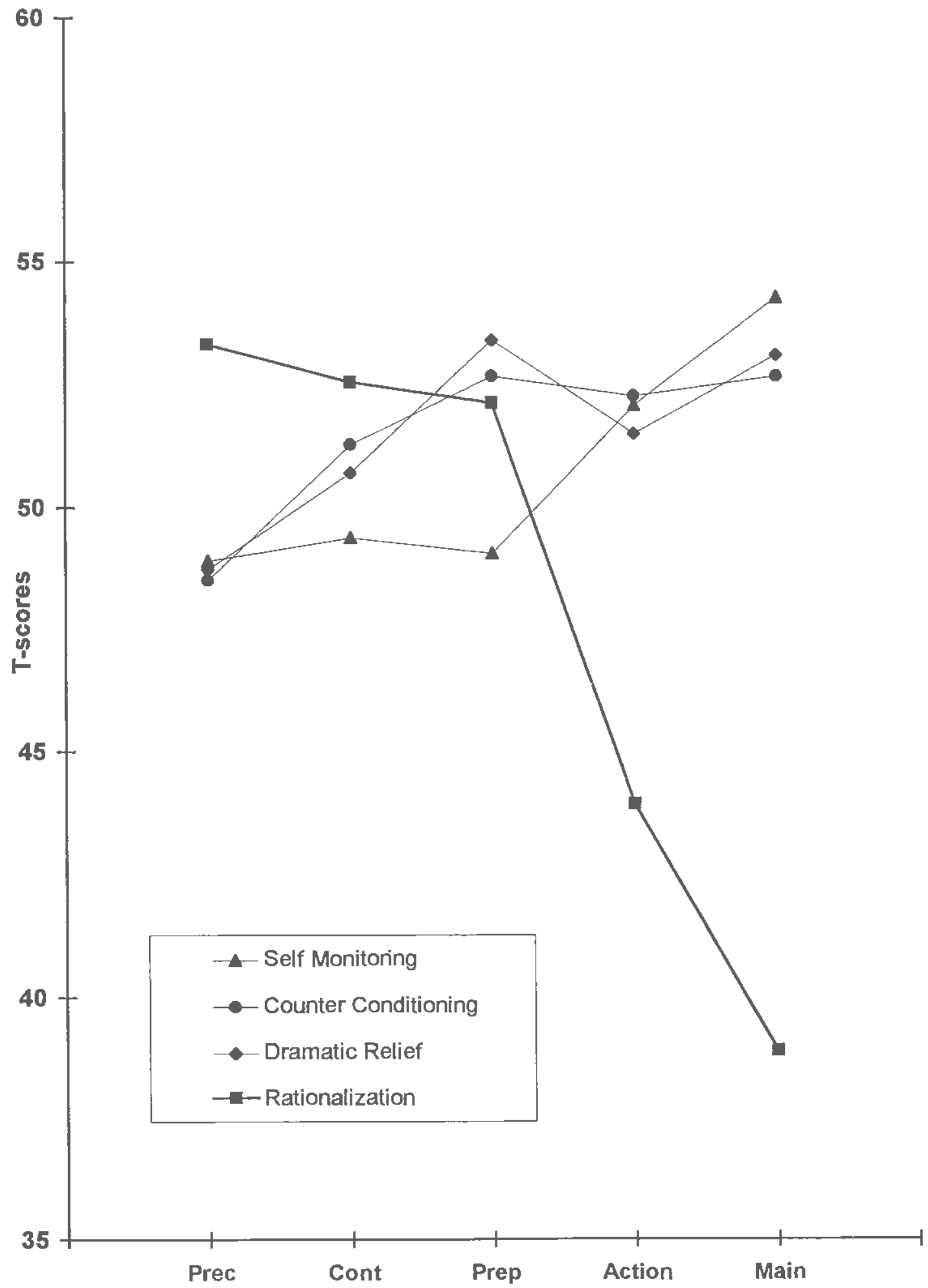




\section{PART VII. OVERALL DISCUSSION}

The investigation presented in the preceding manuscripts constitutes an initial application of the main constructs of the Transtheoretical Model to immoderate drinking by college students. As an initial investigation, much of the analysis was directed at developing psychometrically sound and valid measurement instruments. This is a necessary first step in applying this model to a new behavior and population. This research also provides much information on the nature of college drinking, how this is the same or different from other behaviors investigated using the Transtheoretical Model, and supplies many general and specific research questions to be pursued in future research. The following discussion will be separated into four sections: scale and model development, college drinking, implication for interventions, future research.

\section{SCALE AND MODEL DEVELOPMENT}

This study hypothesized the existence of 26 separate components within four separate measurement instruments. Only two of the 26 scales were not successfully measured in any form, namely the Action component of the URICA-A and Social Liberation process. Additionally, two pairs of theoretically related variables did not separate in this data: The Contemplation and Preparation scales of the URICA-A and the Stimulus Control and Interpersonal Systems Control processes. Both of these findings are minor failures to achieve ambitious measurement goals. One hypothesized component split into two components. The Cons of Immoderate Drinking was developed as the Cons-A and Cons-P, although the unitary Cons component was also a sound solution. One scale was not hypothesized and was found somewhat unexpectedly, namely the Social Anxiety scale of the Temptation instrument. Only one of the final 24 scales did not have significant differences across the Stages of Cessation, and this result also provides validity for the Stage of Change variable. A few of these scales 
could be improved psychometrically, most notably the Precontemplation, Cons-A, and Denial/Repression scales. Yet even these weaker scales had strong relationships with the other constructs investigated.

A number of the scales investigated in this study had not been studied as part of the Transtheoretical Model previously. These include all the Processes of Resistance and the Self M̄onitoring Process of Change. The former provides a new dimension to the Processes construct that will be useful in understanding how individuals resist the forces that promote behavior change. This dimension not only provides a link with more traditional psychodynamic concepts, but might be important in designing intervention programs. The Self Monitoring process was based on intervention programs aimed at developing controlled alcohol use (Miller, 1987) and promises to be adaptable to other behaviors that involve reducing, but not eliminating a behavior. A major area of application might be in the area of diet.

Overall, these instruments represent a set of interrelated variables that has been organized into four separate constructs. The Stages of Change variable, as measured algorithmically or with clusters based on the URICA-A, is the heuristic that organizes the other variables into an integrated whole. The generally strong relationship with Stage found in this investigation for the three other constructs, demonstrates that the structural integrity of the Transtheoretical Model was preserved in these data on college drinking.

One anomalous finding was a consistently low level of Process of Change use for those in Action. This prompted further investigation of the criteria used to classify subjects. It was discovered that the addition of an intentional criterion to remove students planning to return to immoderate drinking eliminated this anomalous finding. Although this result should be interpreted with caution, it is very interesting. Possible motivations for students to reduce with the intention to increase their drinking were proposed in Study 5. Other questions of interest include why this anomaly was not seen for most other model and alcohol consumption variables. One hypothesis is that the group of students removed from the Action and 
Maintenance stage are less involved than others in their drinking, and therefore they can easily drink less when there is a temporary reason to do so. It would be expected that such students would have low Temptations and Pros scores, explaining the findings that are consistent with being in Action, but that they would not use the Processes of Change greatly, resulting in the anomalous findings for these scales.

Another question is why this finding was found in this behavioral domain and not previously, given the large body of research using the Transtheoretical Model. It might be an attribute of the type of behavior investigated, the controlled use of a psychoactive substance, which is unique within the behaviors investigated with the Transtheoretical Model. Alternatively, it might be a phenomenon that is an interaction of the behavior and the population investigated. Further investigations attempting to delineate the characteristics of the removed subjects or studying the behavior of controlled drinking for other populations would help answer these questions.

\section{COLLEGE DRINKING}

This research provides an overview of college drinking as seen through the results of the instruments developed and measured in this investigation. The behavior that was focused on was immoderate drinking, defined as the fairly regular ingestion of either four or five drinks depending on gender. This behavior occupies the middle ground on a chronic/acute dimension. It is occasional, in that engaging in it once a week, during most but not necessarily all weeks is the criterion used. This is in contrast to clearly chronic behaviors such as smoking and excessive diets. Yet, this behavior is much more chronic than other risky behaviors, such as unsafe sex or experimenting with illicit drugs. The regular but not continuous nature of this behavior will have implications for how it is maintained and changed. For example, it is reasonable that a Habit Strength temptation subscale would not be found because this largely weekend behavior is likely more under social than physiological control. 
An important finding of this research is the distribution of students across seven different groups. Although the majority of students were heavy drinkers, substantial numbers were in the three non-problem drinking groups, Precontemplation for Acquisition, and Action and Maintenance for Cessation. Furthermore the stage variable separated the immoderate drinkers into four stages, Action for Acquisition, and the Stages of Cessation of Precontemplation, Contemplation, and Preparation. This classification paradigm separates students into meaningful groups that show substantial differences on outcome and attitude variables and are likely to be predictive of future behavior. Although not fully investigated in this study, cluster analytic techniques separated the largest group, Precontemplators for Cessation, into three subgroups that also might prove meaningful.

What is also evident from this research is that a substantial number of college students, especially those in Contemplation, Preparation, and some of those who have reduced their drinking are quite ambivalent about their and others' alcohol consumption, Many use both Processes of Change and Processes of Resistance, which, although hypothesized to operate in opposite directions, are not substantially negatively correlated. Their Pros and Cons scores are in near balance, and they make heavy use of Self Reevaluation. This ambivalence may reflect the ambivalence within society, especially on the issue of abstinence. This issue can be discussed within the Decisional Balance paradigm. Although the Pros measured in the instrument developed in Study 2 are largely assessments of the pleasures of intoxication, one might hypothesize other positive effects of drinking that are unlikely to be recognized by students, but which might be important. These include acculturation, improvements in self-control by practice with intoxication, and the positive effects of the lowering of inhibition that allows for the experimentation with new behavior. Research on high school students has shown that those who use experimental amounts of alcohol or marijuana tend to be better adjusted than those who abstain or use these substances excessively (Jones, 1968, 1971; Shedler \& Block, 1990). The recognition of these benefits does not lessen the seriousness of the negative consequences associated with drinking, or the 
importance of helping college students gain control of their drinking, but it does put these efforts in a larger context, and perhaps helps to explain why heavy drinking is so prevalent.

Another attribute of college drinking that is clearly demonstrated in this research is how interpersonally mediated this behavior is. The Helping Relationship process, although not showing meaningful differences across the Stages of Change, was one of two processes with population means much higher than the other processes. Furthermore the Temptation instrument suggested that there are at least three types of interpersonal experience that promote drinking differentially for college students. They are: positive and social aspects, peer pressure, and those experiences that produce interpersonal anxiety for college students. These scales had strong correlations with the Pros, which strongly assessed the interpersonal benefits of drinking. The strength of interpersonal influence is also seen in the Stimulus Control scale, which is dominated by items measuring students' efforts to control interpersonal cues to drink.

It is of interest that this facet of college drinking is so clearly evidenced by a model that was initially based on examining the use of intrapsychic processes to change behavior, with Helping Relationship being an obvious exception. This may represent both a strength and a weakness of the model for this behavior. It suggests that the model has the flexibility to intersect with meaningful aspects of a behavior even if the behavior is strongly influenced by the interpersonal environment. The question remains whether intervening on these aspects will have meaningful affects on this behavior. Only research that tests model-based interventions will ultimately answer this question.

\section{TRANSTHEORETICAL MODEL BASED INTERVENTIONS}

Interventions that aim at modifying immoderate drinking for college students can have a variety of goals and methods of administration. This investigation has focused on the cessation of immoderate drinking, and provides only limited information applicable to primary prevention. The investigation of the Stages of Acquisition suggests that only a small amount 
of Acquisition is occurring in this population. What it does suggest is that to optimize primary prevention efforts, they should be aimed at freshman living in dormitories, especially women. Traditionally, prevention has been done in group or population formats. Of interest is a new attempt using the Transtheoretical Model to do primary prevention for adolescent smoking acquisition in an individual format (Pallonen, Velicer, Bellis, Tsoh, \& Smith, 1994). This intervention uses a personal computer based expert system, which gives the user individualized feedback on their Stage, Decisional Balance, and Temptation status, with suggestions for improvement. The present study provides not only Decisional Balance and Temptation scales for use in such an expert system but also a set of Processes that are likely to be applicable to moderate drinkers who are in acquisition. Further attention is needed to measure the Contemplation and Preparation of Acquisition stages.

There are clear precedents for using the Transtheoretical Model to help individuals cease an unhealthy behavior and prevent relapse (Prochaska, DiClemente, Velicer, \& Rossi, 1993). These interventions have used an expert system to give individualized feedback with the goal of moving subjects to the next stage. Variables which have high use in a stage or in the next stage are chosen as applicable for feedback for that stage. The present investigation suggests that an analogous program for immoderate drinkers could be built without many needed adjustments. Possible changes include the use of Self Monitoring for those in Action and Maintenance, the use of Environmental Reevaluation as a late stage process, and giving feedback on the Processes of Resistance. The latter will be especially interesting, as these processes come from a therapeutic tradition that asserts that resistance is unconsciously motivated and usually can not be changed by simple educational means.

Stage-matched group interventions based on this schema might also prove effective and would be much less costly to develop than expert systems. Stage specific manuals have been used in the past for smoking, and could be developed for immoderate drinking. Interventions on a population scale are more difficult to design, as a basic tenet of Transtheoretical Model based interventions is that they are stage specific. However, 
consistent with the findings of this study, interventions that aim at lessening the importance of the Pros of drinking would likely help many college students progress to more advanced stages (or help those in Acquisition not to progress). Such interventions would be consistent with those designed on the basis of alcohol expectancy research (Baer, Marlatt, Kivlahan, Fromme, Larimer, \& Williams, 1992).

\section{FUTURE RESEARCH}

Through the course of this investigation a number of suggestions for future research have been made. The complexity of the behavior being examined, along with the richness of the data gathered, combined with the explanatory potential of the Transtheoretical Model result in many promising avenues for future research. The following is a brief discussion of some possibilities.

Replication of the results found in this study would be necessary before they can be confidently accepted. This is especially true for the finding that an additional intentional criterion for the stages of Action and Maintenance of Cessation improves theoretically predicted and previously confirmed stage characteristics. This finding is based on a post-hoc investigation and is a departure from a large body of previous research on the stage construct making replication a necessary step before this result can be generalized beyond the present sample. Other findings that should be interpreted with caution until replicated, include the patterns of results across stages that are not based on significant pair-wise differences. Also the Processes of Change are based on an unreplicated, exploratory procedure that is possibly affected by sample error. The three factor solution of the Decisional Balance construct was an unexpected solution that needs to be reproduced. Also, replication on a more representative sample would increase the confidence in the generalizability of these findings. More cross-sectional research would also allow for the improvement of measurement scales, by either the addition of new items to strengthen existing scales, or the testing of whole new 
sets of items to assess more salient aspects of certain constructs (e.g. Environmental Reevaluation and Helping Relationship).

There are other areas that have received little or no attention in this study. The acquisition of immoderate drinking was only minimally assessed. There is a sizable number of students in the Precontemplation stage of Acquisition, and understanding how they differ from other students on model variables would provide useful information. It would be especially interesting to understand how this group differs from Maintainers, as both are drinking moderately. Does a history of heavy drinking change how present consumption is managed?

A construct not investigated is the Levels of Change. Understanding what college students see as influencing their consumption patterns would allow a more thoughtful designing of interventions. Do students see drinking as largely interpersonally determined, as the present investigation suggests? Investigating this construct would complete an investigation of all the important model constructs.

After the establishment of sound measures of the constructs of this model, more integrative analysis could also be attempted. This study has clearly demonstrated the structural relationship of model constructs to Stage of Change. There are many other aspects of inter-construct relationship that could be investigated. One implicit causative model is that use of the Processes of Change leads to Stage movement which leads to behavior change. This should be empirically tested. Other questions include how the Decisional Balance and Temptation constructs causally fit into this model. Although initial analysis of models can be made with cross-sectional data, longitudinal data is necessary for a full investigation of these questions.

Longitudinal data would be useful for understanding the predictive utility of individual constructs as well. Random assignment intervention studies would also allow an assessment of how easily modified these variables are, and to what extent these modifications affect behavior. Many of the variables assessed in this model have a stability between states and 
traits. Such variables are open to modification, much more so than other traditionally assessed variables, such as gender, SES, and family history.

Although the investigation of the psychosocial development variables did not provide a clear picture of the interaction between these variables and model constructs, it is felt that additional investigation into the relationship to the Transtheoretical Model of these and other variables that affect behavior will be useful. Such investigations allow for the placing of this model into a larger context and the improved understanding of the model's strengths and limitations. Immoderate drinking by college students is a fascinating, complex behavior that would provide rich data for the investigation of these questions. 


\section{REFERENCES}

Baer, J. S., Marlatt, G. A. Kivlahan, D. R., Fromme, K., Larimer, M.E. \& Williams, E. (1992). An experimental test of three methods of alcohol risk reduction with young adults. Journal of Consulting and Clinical Psychology, 60, 974-979.

Jones, M. C. (1968). Personality correlates and antecedents of drinking patterns in adult males. Journal of Consulting and Clinical Psychology, 31, 1-12.

Jones, M. C. (1971). Personality antecedents and correlates of drinking patterns in women. Journal of Consulting and Clinical Psychology, 36, 61-69.

Miller, W. R. (1987). Techniques to modify hazardous drinking patterns. In M. Galanter (Ed.), Recent developments in alcoholism, (Vol. 5. pp. 425-438). New York: Plenum.

Pallonen, U. E., Velicer, W. F., Bellis, J. M., Tsoh, J., \& Smith, N. F. (1994, April). A computer-based expert system for smoking prevention and cessation in adolescents. Paper presented at the 15th annual meeting of the Society of Behavioral Medicine in Boston, MA.

Prochaska, J. O., DiClemente, C. C., Velicer, W. F., \& Rossi, J. S. (1993). Standardized, individualized, interactive and personalized self-help programs for stages of smoking cessation. Health Psychology, 12, 399-405.

Shedler, J., \& Block, J. (1990). Adolescent drug use and psychological health. American Psychologist, 45, 612-630. 
APPENDIX A: Survey Instrument 


\section{ALCOHOL SURVEY}

1. SEX: (1)__Fenale or (2)__Male

2. CURRENT URI STATUS: (1) Freghman (2) Sophonose (3) Junior

(4)___senior (5)__ Fifth-year Student (6) __ Non-degree student

3. AGE:

years and months

4. RACE: (1) Asian (2) Black (3) Hisparic (4) (5)_White (not Hispanic) (6) other

4) American Indian

5. CURREMT GUMULATIVE GRADE POINT AVERAGE (GPA):
(1) $-4.0-3.6$
(2) $3.5-3.1$ (3)
(6)__ 1.5 or legs (7) _ I do not have a GPA yet
-2.6 (द) $2.5-2.1$

6. CORPEHILY LIVING IN: (1)__dorm (2)__sorority / fraternity (3)__ rented apt+/home (4) parents home (5) own home

7. PARENTS OR LEGAL GUARDIANS LIVE:

(1)__ in Rhode Island (2)__ in another state (3) __in another country

8. YOUR HEIGHT: feet inches

9. your WEIGHT: pounds

For all the questions of this survey think of one drink as one regular beer $(12 \mathrm{oz} .1$, one glass of wine (4 oz.), one single mixed drink, one shot of hard liquor ( 1 oz.), or one wine cooler.

10. In a typical month of 30 days, on how many days do you have at least one drink: containing alcohol? days

11. How many drinks do you have on a tyrical day when you are drinking? drinks.

12. How many drinks do you typically drisk on each day of the week? fput 0 if you do not usually drink on a certain day of the week)?

_ drinks on Monday
_rinks on Tuesday
_ drinks on Wednesday
_ drinks on Thurgday

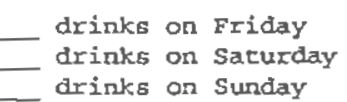

- drirks on sunday

13. During the last 30 days what is the hiqhest number of drinks that you drank on any one occasion. drinks

14. I start to feel the effects of alcohol after consuring drinks.

15. I start to feel intoxicated after consuming drinks.

16. I am uswally drunk after consuring drinks .

17. How old were you when you began to drink without supervision? years ol.

I have never drunk without supervision

18. How old were you the first time you got drunk? years old _ I have never been drunk 
In answering questions 19-22: Think of the six month period of your life that you drank the most alcohol. (If you have never drunk alcohol put NA for questions 19-221

19. How old were you at the beginning of this period? years and months

20. In a typical month of 30 days during this period, on hos many days did you have at least one drink containing alcohol? days

21. On average how many drinks did you consune on daye that you drank? drinks

21. In a troical week during this period did you usually consume the following amounts on one or more occasions?

$$
\begin{aligned}
& 4 \text { or more drinks Yes No } \\
& 5 \text { or more drinks }=\text { Yes }
\end{aligned}
$$

23. Presently, in a typical week do you usually have 4 or more drinks on one or more occasions?

(1)
(2)
only, A and $\mathrm{B}$
How long have you been typically drinking
4 or more drinks one or more times a week?
(1)__Less than 3 months
(2)__between 3 and 6 months
(3)__between 6 months and a year
(4)__ more than 1 year

B. Do you plan to stap typically drinking 4 or mare drinks one or more times a week?

(1) no

(2) Yes, in the next 6 months

(3) _ Y yes, in the next 30 days
(1) yes

2) no $=$ if no, answer oniy $C$ and $D$

C. When did you stop typically drinking 4 or more drinks one or more times a week?

(1) I have never typic ally drunk 4 drinks

(2) I stopped less than 6 months ago

(3) I stopped more than 6 months ago

D. Do you intend to stant typically drinking 4 or more drinks one or more times a week?

(t) Yes, in the next 30 days

(2) Yes, in the next 6 months

(3) No, I am not planning to drink 4 or more drinks one or more times a week

24. In a typical week do you usualiy have $\mathbf{5}$ or more drinks on one or more occasions?

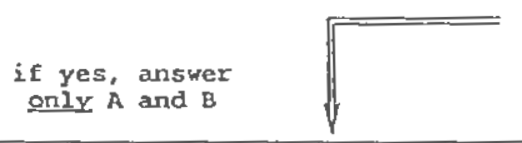

A. How tong have you been typically drinking 5 or more drinks one or more times a week?

(11) Less than 3 months

(2) between 3 and 6 monthis

(3)_between 6 months and a year

(4) more than 1 year

B. Do you plan to stop typically drinking 5 or more drinks one or more times a week?
(1) no
(2) Yes, in the next 6 months
$(31$ _ yes, in the next 30 days

(2)

\section{(1) yes}

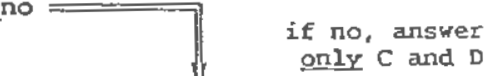

C. When did you stop typicalty drinking 5 or more drinks one or more times a week?

(1) I have never typically drunk 5 drinks

(2) I I topped fess than 6 months ago

(3) I stopped more than 6 months ago

D. Do you intend to start typically drinking 5 or more drinks one or more times a week?

(1) Yes, in the next 30 days

(2) Yes, in the rext 6 months

(3) No, I am not planning to drink 5 or more drinks one or more times a weet. 
For the following questions think of "heavy drinking" or "drinking a lot" as usually consuming 4 or more drinks for women or 5 or more drinks for men one or more times in a typical week.

Please indicate the extent to which you tend to agree or disagree with each statement below. Use the following 5 -point scate:

$$
\begin{aligned}
& 1=\text { Strongly Disagree } \\
& 2=\text { Disagree } \\
& 3=\text { Netutral }- \text { No opintion } \\
& 4=\text { Agree } \\
& 5=\text { Strongly Agree }
\end{aligned}
$$

STRONGLY STRONGLY
DISAGREE AGREE

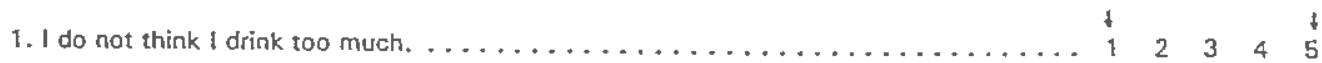

2.1 have just recently stopped drinking heavily. . . . . . . . . . . . . . . . . . . . 1 23445

3. I enjoy my drinking, but sometimes I drink too much. . . . . . . . . . . . . . . . . 1 122345

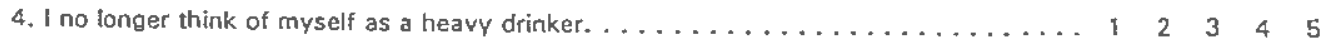

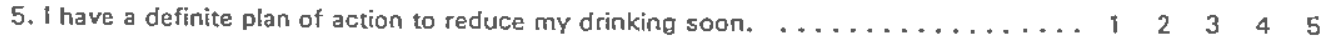

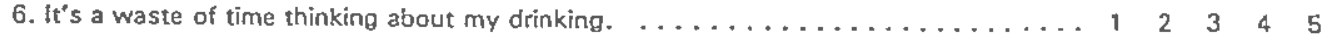

7.1 try not to reconsider my decision to become a light or non-drinker. $\ldots \ldots \ldots \ldots \ldots$. . . $\quad 2 \quad 3445$

8. I am at the stage where I should think about drinking less alcohol. . . . . . . . . . 1122345

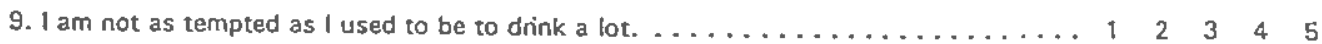

10. Although I have reduced my drinking already I must reduce it more. . . . . . . . . . $1423 \quad 345$

11. Drinking less atcohol woutd be pointless for me. . . . . . . . . . . . . . . . . . i 2 34 5

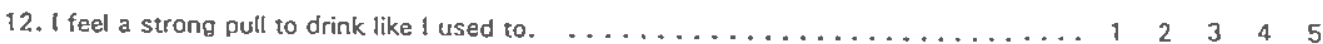

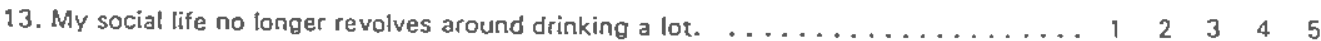

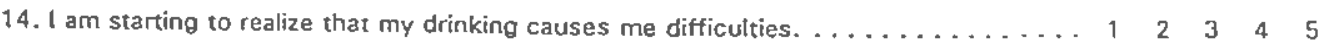

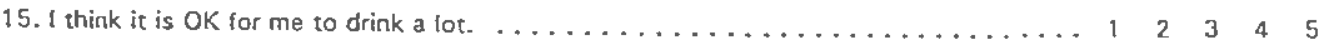

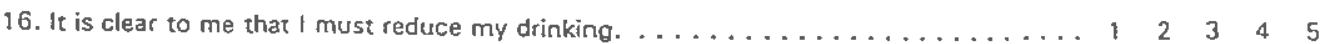

17. Having just changed my drinking habits it would be easy for me to drink heavily again. ................................... 123345

18. Although I still drink a lot it is not as much fun as it used to be. . . . . . . . . . 1 2 234 5

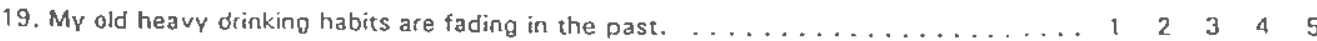




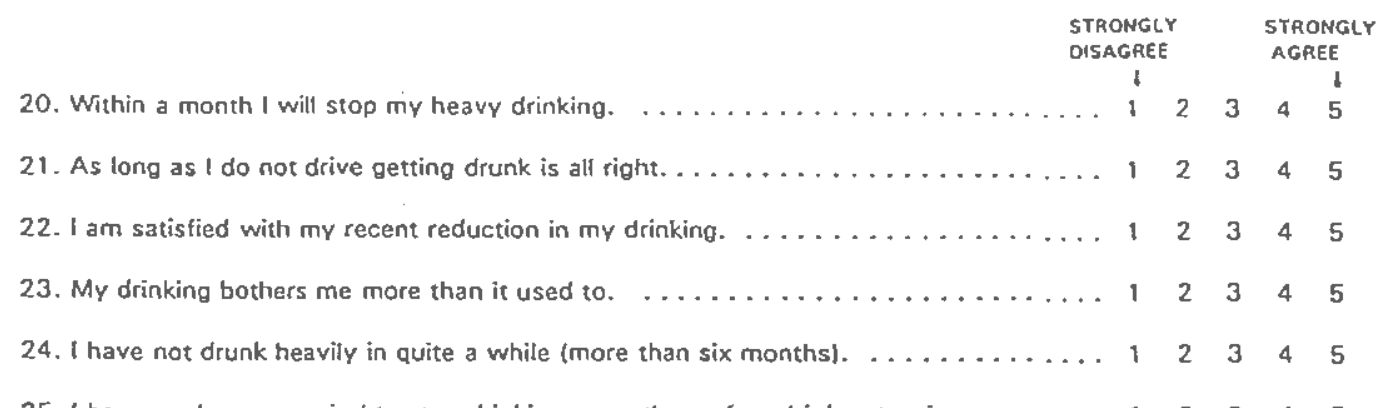

25. I have made up my mind to stop dtinking more than a few drinks at a time. $\ldots \ldots \ldots 4.54$

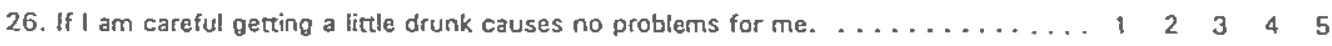

27. I have started to wonder if my drinking is good for me. . . . . . . . . . . . . 142345

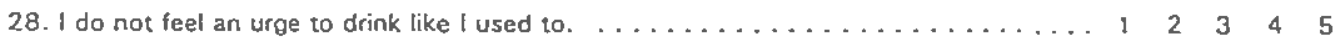

29. Having 4, 5 , or more drinks at a time is ok for me. $\ldots \ldots \ldots \ldots$............ 4

30. Although it is good that 1 am no longer drinking a lot it still feels strange

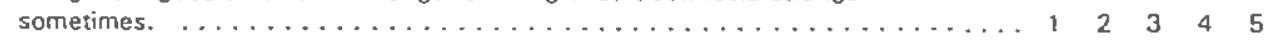

31. 1 am building up my confidence to cut my drinking down soon $\ldots \ldots \ldots 4$

32. I think that maybe I should limit my drinking to no more than 3 or 4 drinks at a

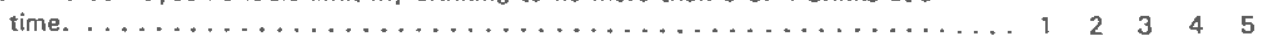

33. I now think of myself as a light or non-drinker. $\ldots \ldots \ldots \ldots \ldots$

34. It's hard to enjoy myself without drinking. $\ldots \ldots \ldots \ldots \ldots \ldots$

35. Sometimes i really miss drinking 4,5 or more drinks on one occasion $\ldots \ldots \ldots 4$

36. Some things in my life would be better if I drank less. . . . . . . . . . . . . . 142345

37. I am making a firm commitment to myself to stop drinking heavily very soon $\ldots \ldots$

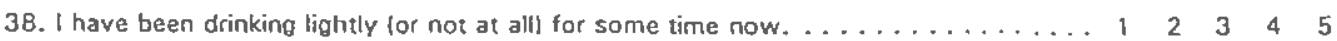


5.

The following experiences can affect the drinking pattern of some people. Think of any similar experiences you may be currently having or have had in the last month. Then rate the FREQUENCY of each event on the following five point scale:

$$
\begin{aligned}
& 1=\text { Never } \\
& 2=\text { Seldom } \\
& 3=\text { Occasionally } \\
& 4=\text { Often } \\
& 5=\text { Repeatedly }
\end{aligned}
$$

NEVER REPEATEDLY

1. I pay close attention to how my body feels when I am drinking. ........... 1 . 2 . 3445

2. Other people treat me better when I do not drink too much. ............. 1 23445

3. I think the bad health effects of drinking will not affect me even if I drink a lot. . . . I $\quad 2 \quad 3 \quad 4 \quad 5$

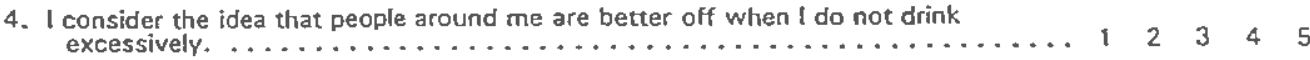

5. I have a non-alcoholic drink instead of a drink contatining alcohol. . . . . . . . . . 1424345

6. I recall information about the benefits of not drinking a lot. . . . . . . . . . . . . 1242345

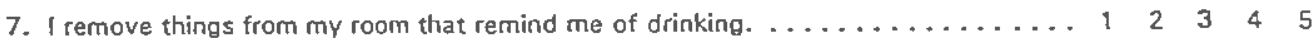

8. I think that my drinking is OK because I will drink less after I graduate from

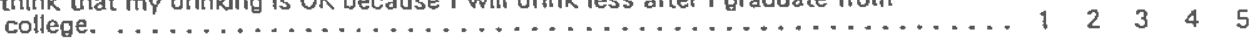

9. I do not like my image of myself as a heavy drinker. . . . . . . . . . . . . . 1122344

10. I am open with at least ane person I can trust about my drinking. . . . . . . . . . . 1 2 2345

11. I get upset when I think about the problems drinking causes . . . . . . . . . . 1 i 2 3 4 . 5

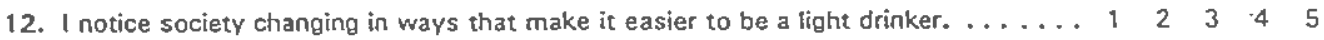

13. Ithink that no-one has the right to tell me how much i can drink. . . . . . . . . 1424345

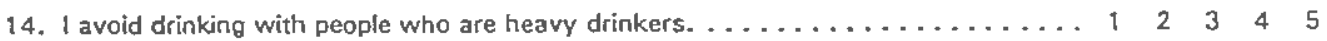

15. I tell myself $i$ can choose to reduce my drinking habits. . . . . . . . . . . . . . . . 1243445

16. I stop or slow my drinking down when I start to feel the effects of alcohol. $\ldots \ldots \begin{array}{llllll}1 & 2 & 3 & 4 & 5\end{array}$

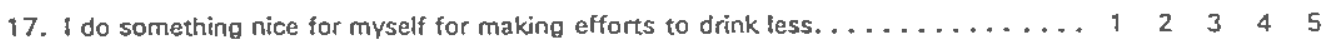

18. I try not to warry about my drinking. . . . . . . . . . . . . . . . . . . . 1 1 23445

19. I stop and think that my dinking is causing problems for others. . . . . . . . . 122345

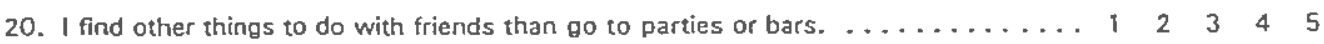

21. I think about information I have heard about problems associated with drinking

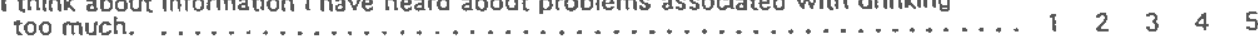

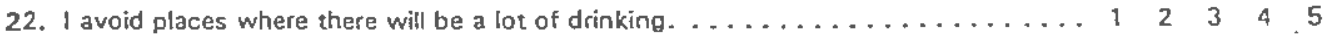

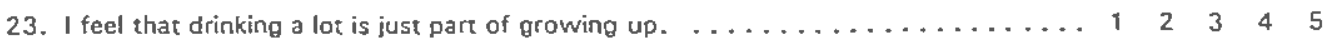

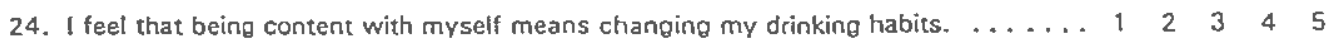

25. I have someane who listens when I need to talk about my drinking. . . . . . . . . $14{ }^{2} \quad 3 \quad 4 \quad 5$

26. Stories about accidents caused by drunk drivers upset me. . . . . . . . . . . . 1 i 23445

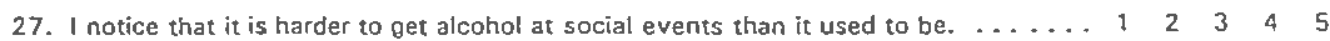

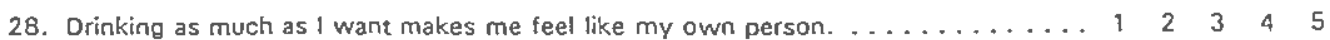

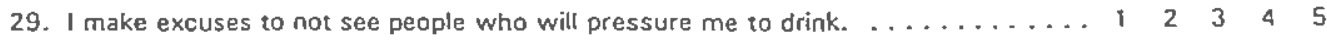

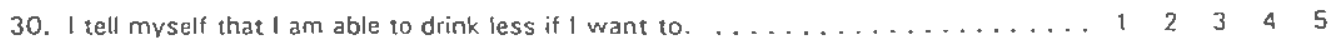

31. I keep track of how many drinks i have had when drinking. . . . . . . . . . . 142345 
- $6-$

NEVER
REPEATEDLY
$\mid$

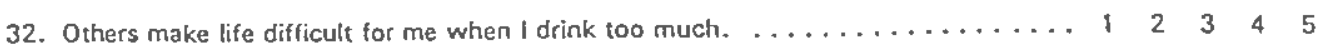

33. I think that even when 1 drink a lot it does not hurt anyone. . . . . . . . . . 142345

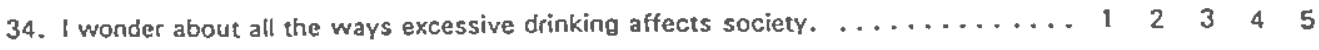

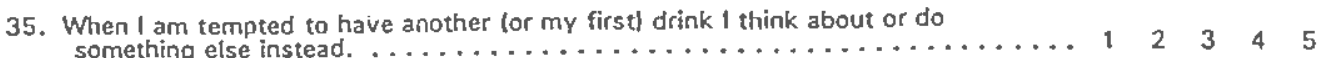

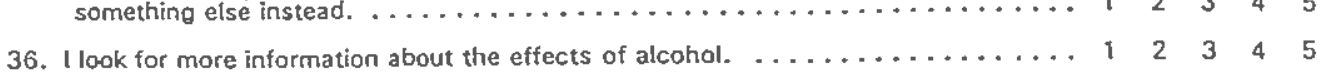

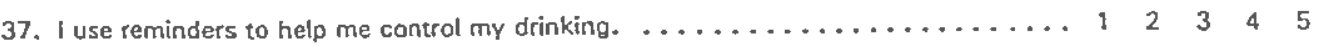

38. I think that drinking with friends has benefits which outweigh the risks. . . . . . 1 i 23445

\begin{tabular}{l} 
39. 1 struggle with the fact that my drinking habits contradict my changing view of \\
myself. $\ldots \ldots \ldots \ldots \ldots \ldots \ldots \ldots \ldots \ldots \ldots \ldots \ldots \ldots \ldots \ldots \ldots \ldots \ldots \ldots \ldots$ \\
\hline$\ldots \ldots \ldots \ldots$
\end{tabular}

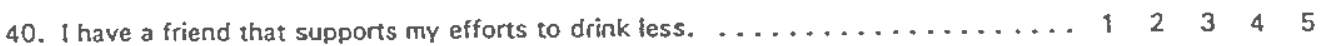

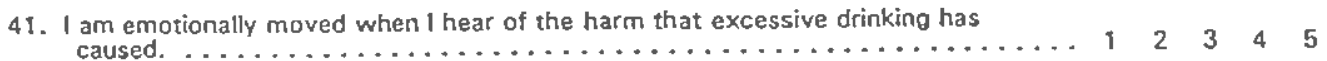

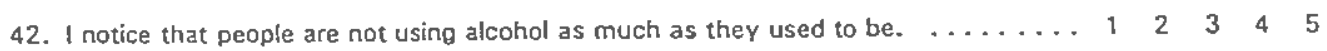

43. Rules saying that I can not drink make me want to drink all the more. . . . . . . . $142 \quad 3 \quad 4 \quad 5$

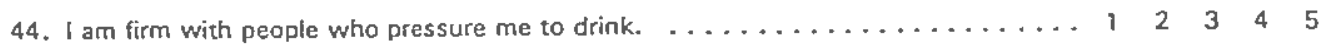

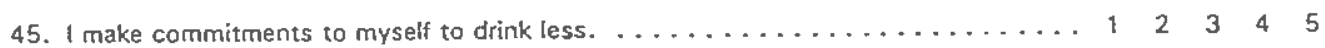

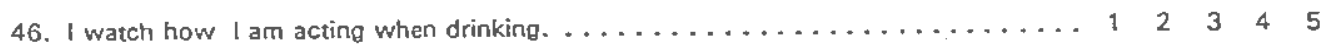

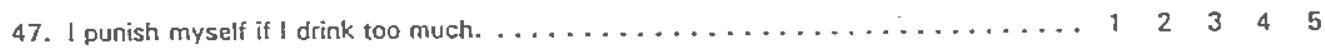

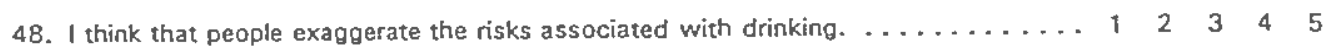

49. I consider the idea that if people drank less the world would be a better place. . . . $1122 \quad 3 \quad 4 \quad 5$

50. I find other ways to relax than drinking a lot. ... . . . . . . . . . . . . . 1424345

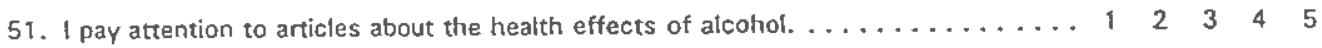

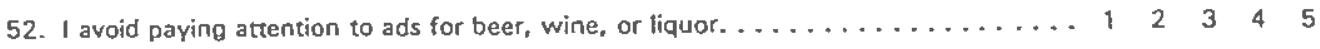

53. I think it is natural to drink a lot when you are in college. . . . . . . . . . . . 1232345

54. I stop to think that I am more productive when I drink less. . . . . . . . . . i 23445

55. I have someone I can count on when I am having problems with my drinking. . . . $1122 \quad 3 \quad 4 \quad 5$

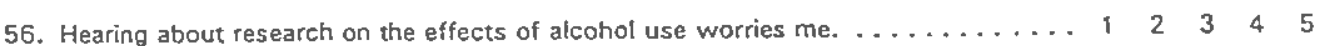

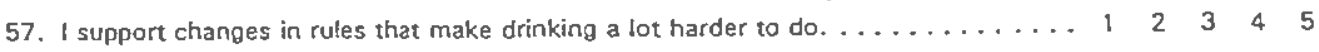

58. I think that it would be silly for me to not drink just because of a rule. $\ldots \ldots \ldots$. $122 \quad 3445$

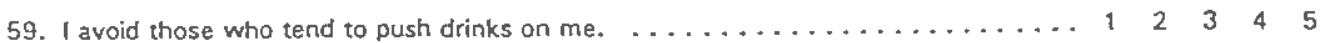

60. I use will power to contral my drinking. . . . . . . . . . . . . . . . . . 1 24345

61. I control my drinking by paying attention to how I an feeling. . . . . . . . . . . 1 2 2345

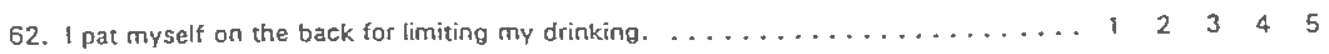

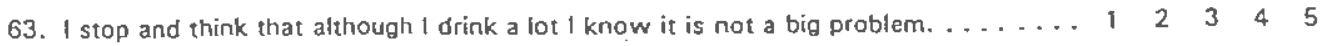

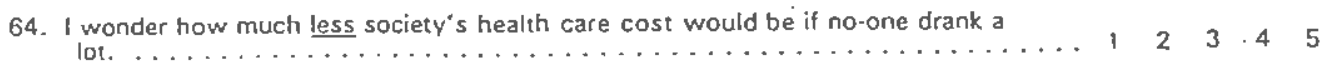

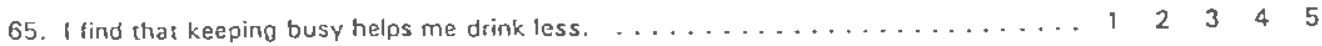




\begin{tabular}{|c|c|c|c|c|c|}
\hline NEV & VER & & & EPE & ATEOLY. \\
\hline 66. I ask other students about their opinions about drinking. . . . . . . . . . . . & 1 & 2 & 3 & 4 & $\begin{array}{l}1 \\
5\end{array}$ \\
\hline 57. I avoid places or events where 1 tend to drink too much. $\ldots \ldots \ldots \ldots \ldots \ldots \ldots$ & 1 & 2 & 3 & 4 & 5 \\
\hline 68. I think that if I did not drink like most others my social life would suffer, ....... & 1 & 2 & 3 & 4 & 5 \\
\hline 69. The way I driak makes me disappointed in myself. $\ldots \ldots \ldots \ldots \ldots \ldots \ldots$ & 1 & 2 & 3 & 4 & 5 \\
\hline 70. I have someone who helps me "see through" my excuses for drinking a lot. . . . . & 1 & 2 & 3 & 4 & $5 !$ \\
\hline 71. Dramatic portravals of the evils of drinking affect me emotionally. $\ldots \ldots \ldots \ldots$ & 1 & 2 & 3 & 4 & 5 \\
\hline 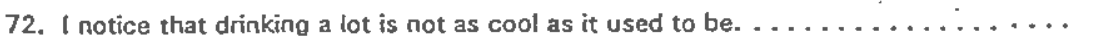 & 1 & 2 & 3 & 4 & 5 \\
\hline 73. I get enjoyment out of getting away with illegal drinking. . . . . . . . . . . . & 1 & 2 & 3 & 4 & 5 \\
\hline 74. I try to spend free time with light drinking friends. $\ldots \ldots \ldots \ldots \ldots \ldots \ldots$ & 1 & 2 & 3 & 4 & 5 \\
\hline 75. I tell myself that 1 will drink less or not at all today. . . . . . . . . . . . . . . & 1 & 2 & 3 & 4 & 5 \\
\hline 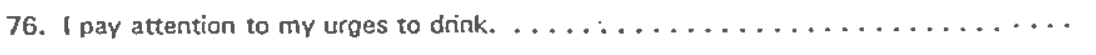 & 1 & 2 & 3 & 4 & 5 \\
\hline 77. Others around me reinforce my not drinking too much. $\ldots \ldots \ldots \ldots \ldots$ & 1 & 2 & 3 & 4 & 5 \\
\hline 78. I stop and think that my heary drinking has not hurt me yet. $\ldots \ldots \ldots \ldots$ & 1 & 2 & 3 & 4 & 5 \\
\hline 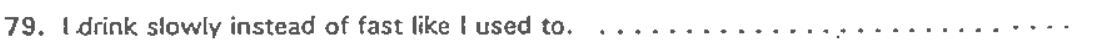 & 1 & 2 & 3 & 4 & 5 \\
\hline 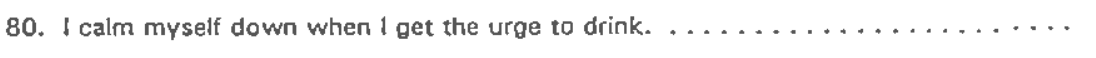 & 1 & 2 & 3 & 4 & 5 \\
\hline 81. I recall information people have given me on the benefits of drinking less. . . . . . & 1 & 2 & 3 & 4 & 5 \\
\hline 82. I do not keep alcohol around where 1 live so $t$ will drink less. $\ldots \ldots \ldots \ldots \ldots$ & 1 & 2 & 3 & 4 & 5 \\
\hline $\begin{array}{l}\text { 83. I think that as a young adult } t \text { am able to handle more alcohol than older } \\
\text { people. } \ldots \ldots \ldots \ldots \ldots \ldots \ldots \ldots \ldots \ldots \ldots \ldots \ldots \ldots \ldots \ldots \ldots \ldots \ldots \ldots\end{array}$ & 1 & 2 & 3 & 4 & 5 \\
\hline 84. I am starting to think that other things are more important than drinking........ & 1 & 2 & 3 & 4 & 5 \\
\hline 85. I get upset when it think about my drinking. $\ldots \ldots \ldots \ldots \ldots \ldots \ldots \ldots \ldots \ldots$ & $\mathrm{A}$ & 2 & 3 & 4 & 5 \\
\hline 86. I react emotionally to the warnings about excessive drinking. . . . . . . . . . . & 1 & 2 & 3 & 4 & 5 \\
\hline 87. I notice that society is trying to help people drink less. . . . . . . . . . . . . & 1 & 2 & 3 & 4 & 5 \\
\hline $\begin{array}{l}\text { 88. My anger at rules that restrict my right to drink makes me want to drink ali the } \\
\text { more. } \ldots \ldots \ldots \ldots \ldots \ldots \ldots \ldots \ldots \ldots \ldots \ldots \ldots \ldots \ldots\end{array}$ & 1 & 2 & 3 & 4 & 5 \\
\hline 89. I a void people who will make me want to drisk. .................. & $T$ & 2 & 3 & 4 & 5 \\
\hline 90. I remind myself that $I$ am able to reduce the amount I drink. . . . . . . . . . & 1 & 2 & 3 & 4 & 5 \\
\hline 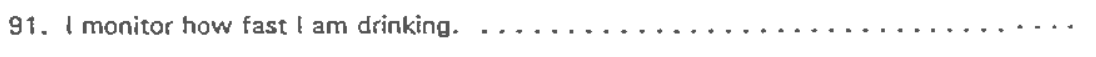 & 1 & 2 & 3 & 4 & 5 \\
\hline 92. I think that the bad parts about my drinking are not really that bad. . . . . . . . . & 1 & 2 & 3 & 4 & 5 \\
\hline 93. I think about what other people have said about how I act when 1 am drinking..... & 1 & 2 & 3 & 4 & 5 \\
\hline 94. I feel that after a hard week at school I deserve to have a good time drinking- .... & 1 & 2 & 3 & 4 & 5 \\
\hline 5. I wonder about my drinking habits. . . . . . & 1 & 2 & 3 & 4 & 5 \\
\hline 6. I assent my autonomy by drinking when I want to. . . . . . . . . . . . . . & 1 & 2 & 3 & 4 & 5 \\
\hline 7. I try to make friends with people who are not heavy drinkers. ............ & 1 & 2 & 3 & 4 & 5 \\
\hline 8. I make efforts to limit my drinking. $\ldots \ldots \ldots \ldots \ldots \ldots \ldots \ldots$ & 1 & 2 & 3 & 4 & 5 \\
\hline I feel that i need the real break from studying or working that drinking can give me..... & 1 & 2 & 3 & 4 & 5 \\
\hline 0 . I seek out people who do things that do not inv & 1 & 2 & 3 & 4 & 5 \\
\hline
\end{tabular}


How IMPORTANT TO YOU are the following statements in YOUR DECISIONS about HOW MUCH

Circle 1 if it is NOT AT ALL IMPORTANT and 5 if it is EXTREMELY IMPORTANT in your decisions. If vour answer is in between use numbers 2,3, oR 4.

NOT AT ALL EXTREMELY IMPORTANT IMPORTANT

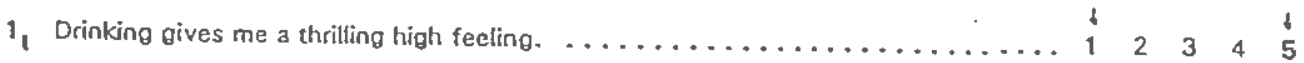

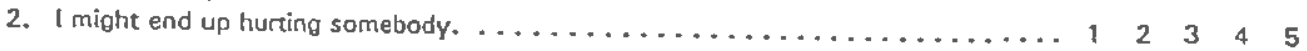

3. I ain mare self confident when i drink. ..................... 1223445

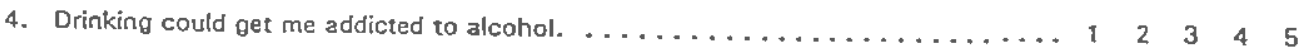

5. Drinking could kill me. ........................... 122345

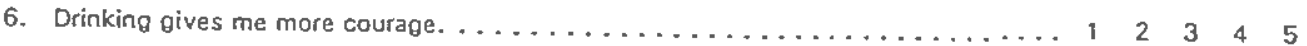

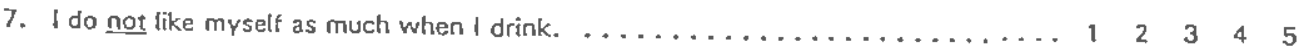

8. Drinking makes me feel mare independent. ................... 123345

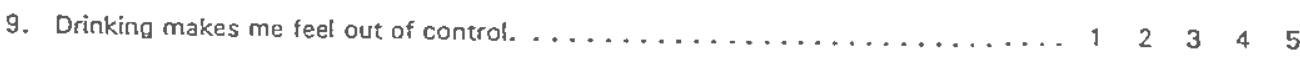

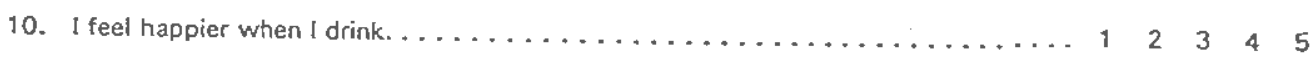

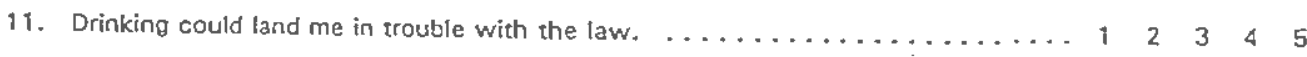

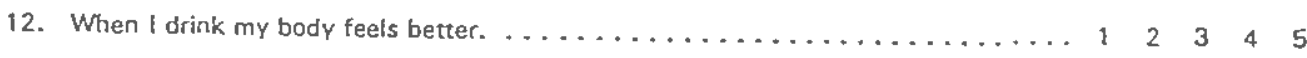

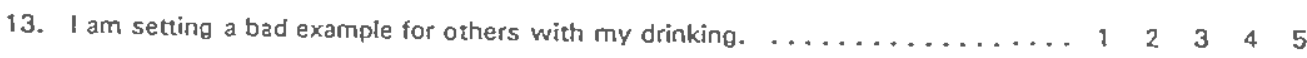

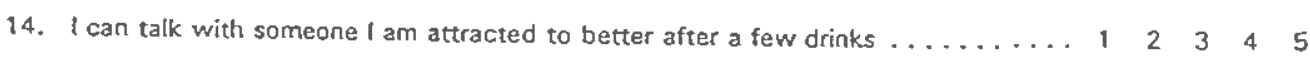

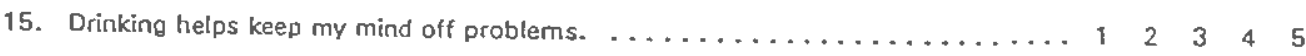

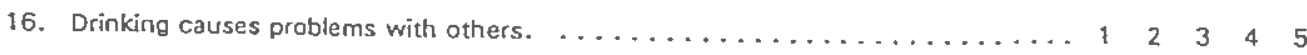

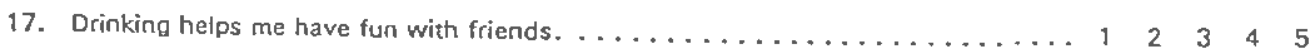

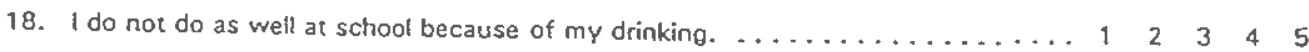

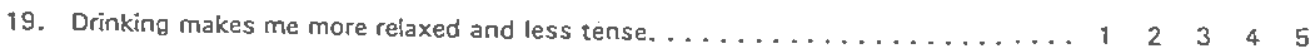

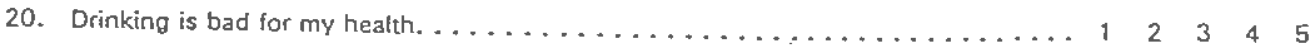

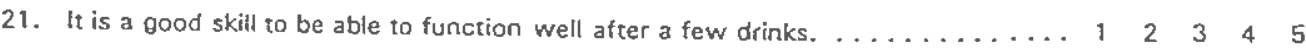

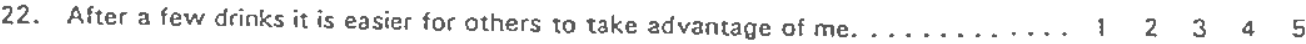

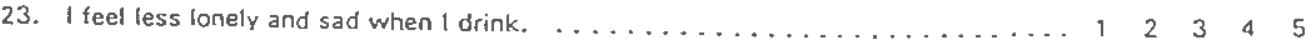

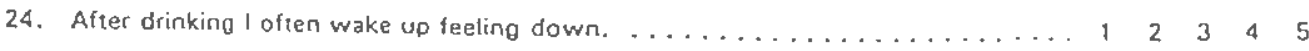

25. Alcohol makes my sexual experiences easier and more enjoyable $\ldots \ldots \ldots \ldots \ldots 1 \quad 2 \quad 3 \quad 4 \quad 5$ 
9 -

Listed below are situations that can lead some people to drink a lot or more than they should. Circle the number that best corresponds to your present feelings of TEMPTATION to drink more than you should in each of the situations below.

$1=\underset{\text { Nempted }}{\text { Not at all }} \quad 2=\underset{\text { Nempted }}{\text { Noty }} \quad 3=\underset{\text { tempted }}{\text { Moderately }} \quad 4=\underset{\text { tempted }}{\text { Very }} \quad 5=\underset{\text { Extremely }}{\text { tempted }}$

1. When I am excited

NOT AT ALL EXTREMELY TEMPTED TEMPTED +234 t

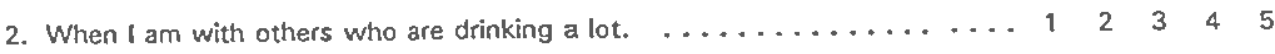

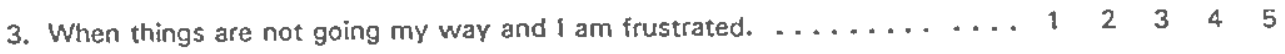

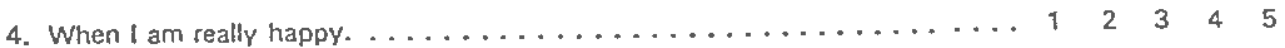

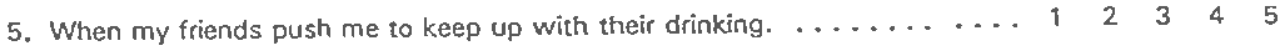

6. When I am feeling depressed. ......................

7. When I am having fun with friends. ................... 1 2 3445

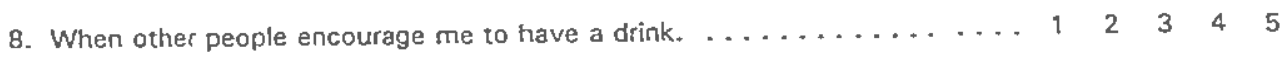

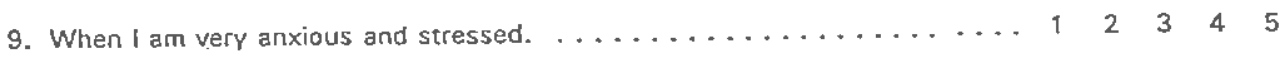

10. When 1 am out on a date. ........................ 1 23445

11. When $\mathrm{l}$ am offered a drink by someone. ................. 142345

12. When 1 am feeling angry. ....................... 1 . 2 3 4 4 5

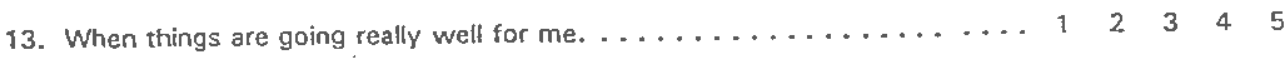

14. If I go to a party where there is a lot of drinking. .............. 1 . 2 . 3445

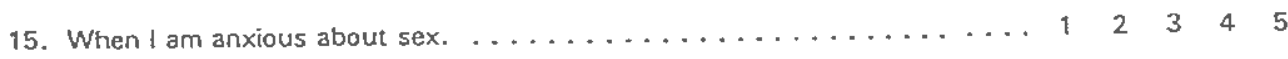

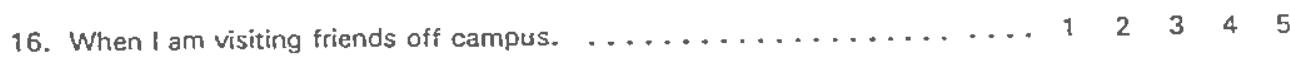

17. When there are drinking games going on. $\ldots \ldots \ldots \ldots \ldots \ldots \ldots \ldots \ldots \ldots$ 1 2 . 34 . 5

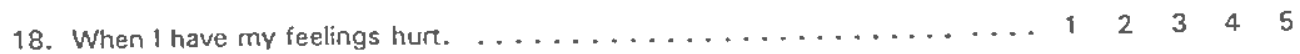

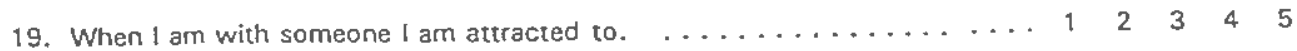

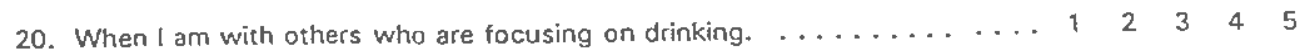

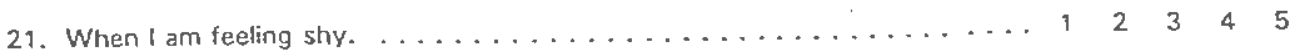

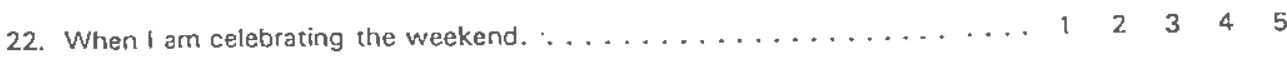

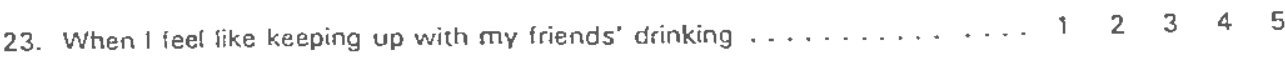

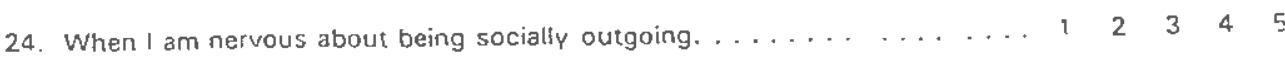


How many times in the last 12 months have you had the lollowing expericnees related to your alcohol consumption?

$\begin{array}{llrl}\text { Please circle the correcl answer } & 0=\text { NEVER } & 3-5=3-5 \text { TIMES } \\ \text { using the following } 6 \text { point seale: } & 1=\text { ONCE } & 6-9=6-9 \text { TIMES } \\ & 2=\text { TWLE } & \geq 10=10 \text { TIMES OR MORE } & \text { NUMBER OF TIMES }\end{array}$

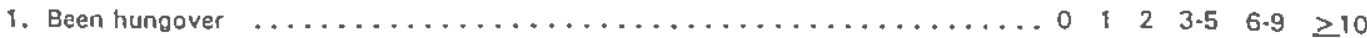

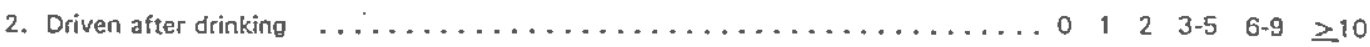

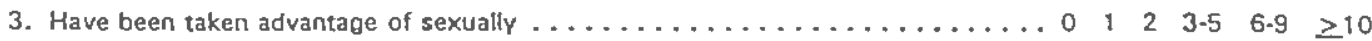

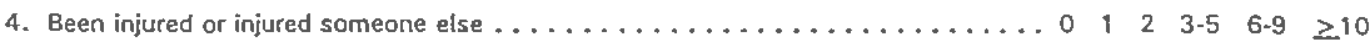

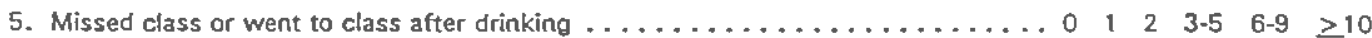

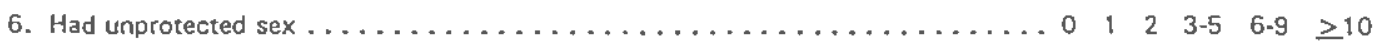

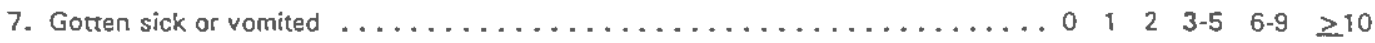

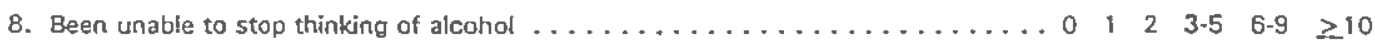

9. Gotten into an argument or fight $\ldots \ldots \ldots \ldots \ldots \ldots \ldots \ldots \ldots \ldots \ldots \ldots \ldots \ldots \ldots$

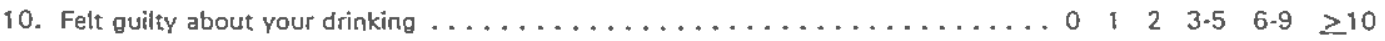

11. Had someone suggest that you cut down on your drinking ............. $012{ }^{2} 3-56-9 \geq 10$

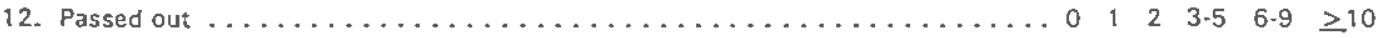

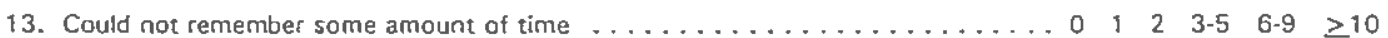

14. Ridden with someone who was driving after drinking . . . . . . . . . . . . $01123.56-9 \geq 10$

15. Needed a first drink in the morning to get yourself going after a heaw drinking

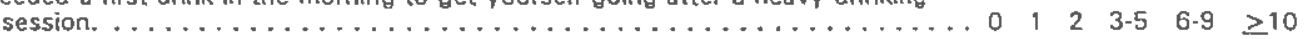

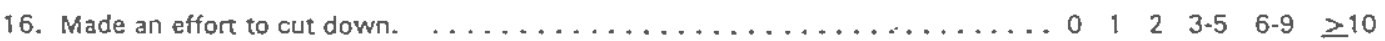

17. Been angry at someone's suggestion that you cut down. . . . . . . . . . . . 0 i 2 3-5 $6-9 \geq 10$

t8. Thought you might have a problem with your drinking $\ldots \ldots \ldots \ldots \ldots \ldots \ldots \ldots .6123-56-9 \geq 10$

19. Been criticized by a date ............................ 0 1 2 . $3-5$ 6-9 $\geq 10$

20 Damaged property ................................ $01123.5 \quad 6-9 \geq 10$

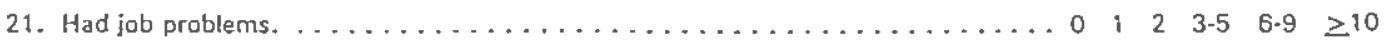

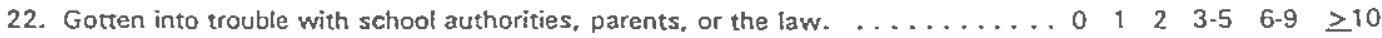

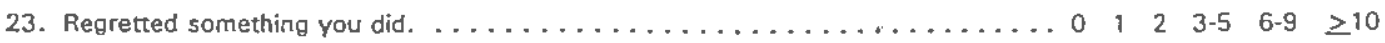

24. Had a problem with a friend or hurt a friendship. . . . . . . . . . . . . o $122 \quad 3-5 \quad 6-9 \geq 10$

25. Been unable to stop drinking once you began. . . . . . . . . . . . . . . . $011223-5$ 6-9 210

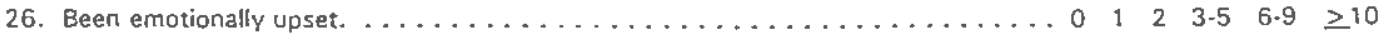

27. Felt low, blue or bummed out the next day. ................. 0 , $23-56-9 \geq 10$

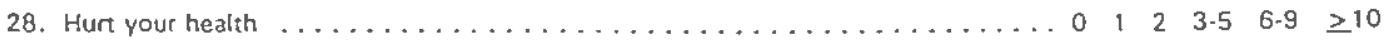

29. Been unable to study, do homewark, or concentrate as you needed to. $\ldots \ldots \ldots .0 \quad 1 \quad 2 \quad 3-5 \quad 6-9 \geq 10$

30. Had a personafity change. ........................... 0 1 2 3-5 6-9 $\geq 10$

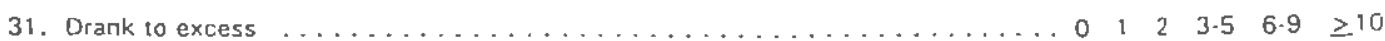


Following is a list of statements that may or may not be true about you. For each statement, please circle the appropriate response using the following 5-point scale:

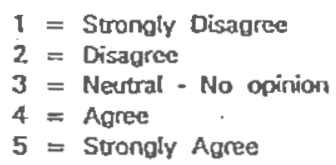

1. It sometimes bothers me if my leisure time activities are different from those of my triends. .................................... 122345

2. I seldom express my opinion in groups if 1 think they will be conteoversial or

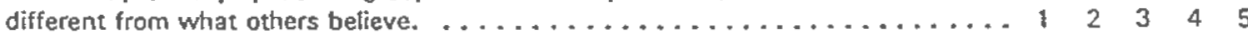

3. I have carefully thought through and decided the extent to which I am involved

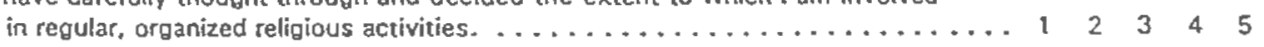

4. I need to feel sure of the outcome before attempting something new or different. .................................. $12{ }^{2} 3445$

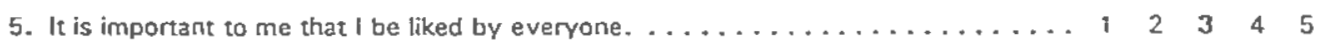

6. I have identified the steps that are necessary for me to take now in order to

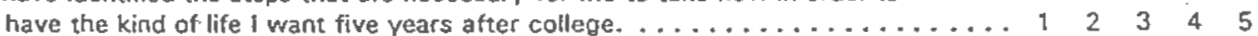

7. I have made a decision about the number of children (including nonel ! plan to have. .................................... 1243445

8. I sometimes hold back my true feelings for a friend because l'm afraid i might

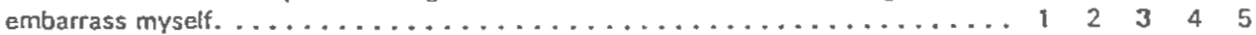

9. 1 find relationships with my close friends not as important to me as they were a vear ago. ................................... 142345

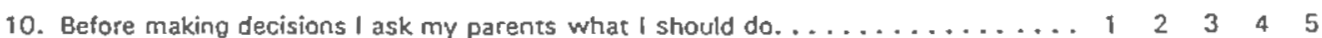

11. I am currently involved in one or more activities that $I$ have identified as being of help in determining what 1 will do with the rest of my life. . . . . . . . . . 122345

14. It is important to me that others accept my point of view. . . . . . . . . . . 1 t 23445

13. I have identified at least three people, other than family members, who I am

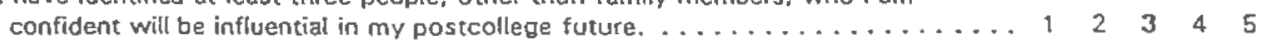

14. I am usually more concerned about the grade 1 will receive than about the

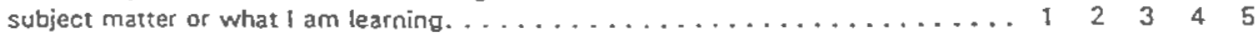

15. Within the past year there have been a number of occasions when I was mistaken about the closeness of a relationship. .................. 1 2 $34{ }^{4} 5$

16. I have one or more goals that 1 am committed to accomplisting and have been working on for aver a year. ........................... $1233^{4} 5$ 

17. I seldom bounce ideas off other people in order to obtain their views of my $\ldots \ldots \ldots \ldots \ldots \ldots \ldots \ldots \ldots \ldots \ldots \ldots \ldots \ldots \ldots \ldots \ldots \ldots \ldots \ldots \ldots \ldots \ldots$
thinking. $\ldots \ldots \ldots \ldots \ldots \ldots \ldots \ldots$

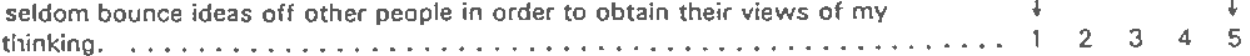

STRONGLY STRONGLY DISAGREE AGREE

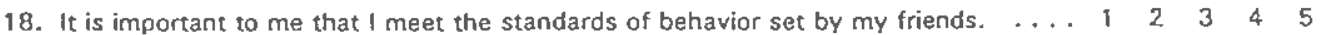

19. The importance I place on things like new cars, large houses, and expensive

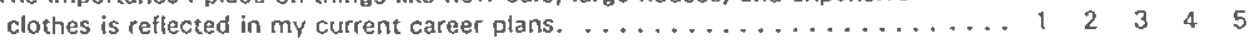

20. When I want to be alone I have difficulty letting my friends know in a way

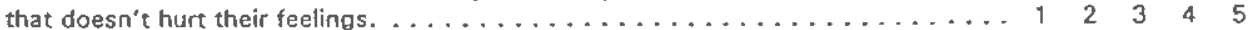

21. An outside, objective observer could readily identify the ethical values that

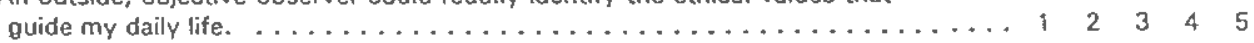

22. I feel guilty when I don't obey my parents' wishes. . . . . . . . . . . . . . . 1 24345

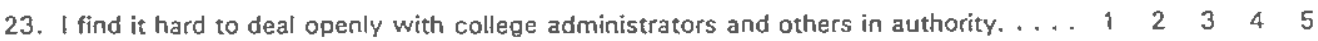

24. Each of my close friends holds at least one view of life or set of personal

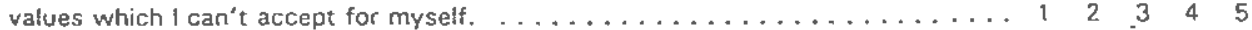

25. I have clearly decided upon the place of marriage and children in $\mathrm{my}$

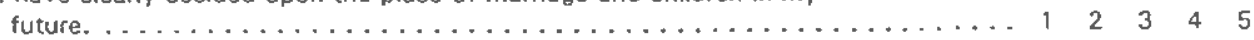

26. Sometimes 1 conceal some of my talents or skills so I will not be asked to

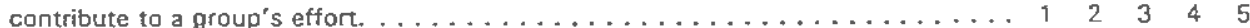

27. After having strong disagreements with a person, I usually try to avoid her/him as much as possible thereafter. ......................... $12{ }^{2} 34 \quad 5$

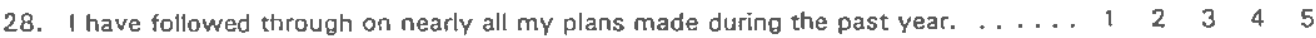

29. I try to keep my friends from knowing about my shortcomings and failures. . . . . . 1 2 23445

30. Decisions about important matters are largely based on what my parentist think and believe. . . . . . . . . . . . . .

31. I can state clearly my plan for achieving the goals I have established for the next ten years. ............................... 1 23445

32. Because of my friends" urgings I sometimes get involved in things that are not in my best interest. ............................ 123345

Do vou plan to significantly reduce the amount of alcohol you drink? (check one)

Yes, in the next 30 days
Yes, in the next 3 months
Y Yes, in the next 6 months
Y Yes, in the next year

Yes, in the next 2 years

Yes, in the next 3 years

- Yes, in the next 4 years

- Yes, in the next $5=10$ years

_ Yes, sometime after the next 10 years
- No, never.

I do not drink at all

THANK YOU FOR YOUR TIME AND EFFORT 
APPENDIX B: Consent form 
Dear Prospective Research Participant;

I am asking you to take part in a research project that I am conducting. Picase read the following description so you can make an informed decision whecther to participate or not. Feel frec to ask questions. If you have more questions later you can call Jeffrey Migneault (792-5568) or Dr. James Prochaska (792-2830).

This projoct is a study of attitudes and bchavion related to alcohol use and other health behaviors. If you participate you will be asked to complete a paper and pencil survey. The questions will focus on your knowledge, attitudes, and behavior. Responses from these items will be collected from men and women students attending the: University of Rlode Island.

You must be 18 years or older to participatc.

If you decide to take part in this study, your participation will involve filling in a survey of items peraining to your experience. This will take 30-45 minutes to complete. Upon completion of the survey. you will return it to the researcher.

The possible risks or discomforts of the study are minimal, possibly some degree of discomfort in providing answers to these questions.

The possible benerits from this study include increased knowledge about your personal behavior in this area. In addition, the researcher hopes to learn valuable information about behaviors and attitudes about alcohol use and other health related behaviors.

Your participation in this study is confidential. None of the actual responses to the survey will identify you. All surveys will be collected anonymously. Information from the study will be reported in grouped data that does not reveal the specifics regarding any individual.

Participation in this study is not expected to be harmful or injurious to you. However, if this siudy causes you injury, you should write or call the University of Rhode Island's Dircetor of Research, 70 Lower College Road, University of Rhode Island, Kingston, RI 02881 (792-2635).

The decision whether of not to take part in this study is up to you. You do not have to paricipate. If you decide to take part in this study, you may quit at any time. Whatever you decide, you will in no way be penalized. If you wish to quit, you simply inform the researcher of your decision. If you are not satisfied with the way that the study is performed, you may discuss your complaints with Jeffrey Migneault (792-5568) or Dr. James Prochaska (792-2830), anonymously if you choose.

You must be 18 years old or older to participate in this research. If vou trave any question about the above description please ask thern now.

Thank you very anucil for your time and effort.

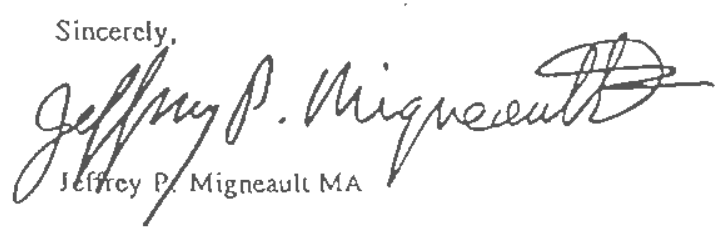


APPENDIX C: Two component Decisional Balance solution by Stage of Change 
Figure C-1: Decisional Balance by Stage of Change

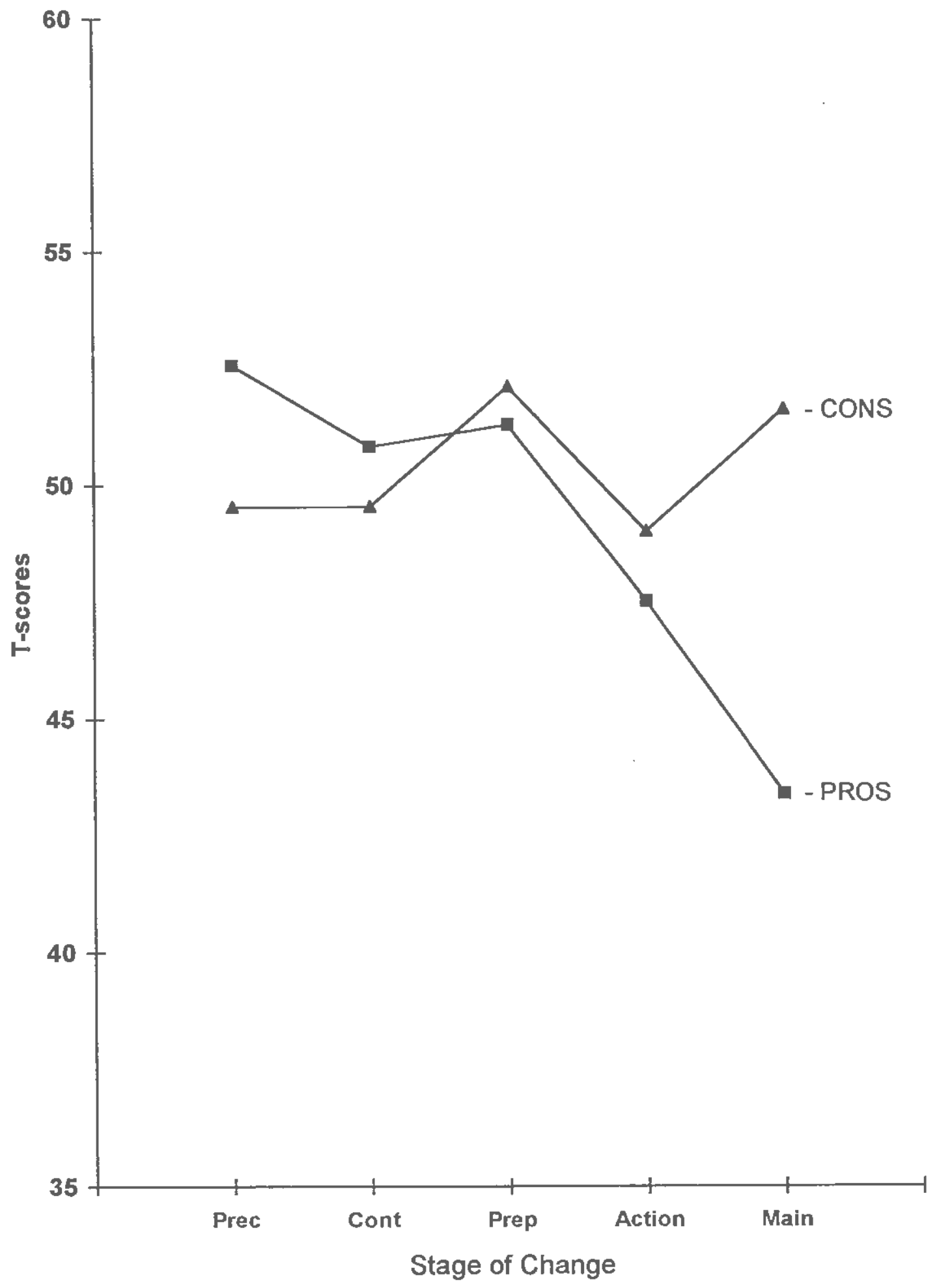

Note: ANOVA for Cons was not significant $(\underline{F}(4,408)=.97, \underline{p}>.05)$ 
Figure C-2: Decisional Balance scales by Adjusted Cluster

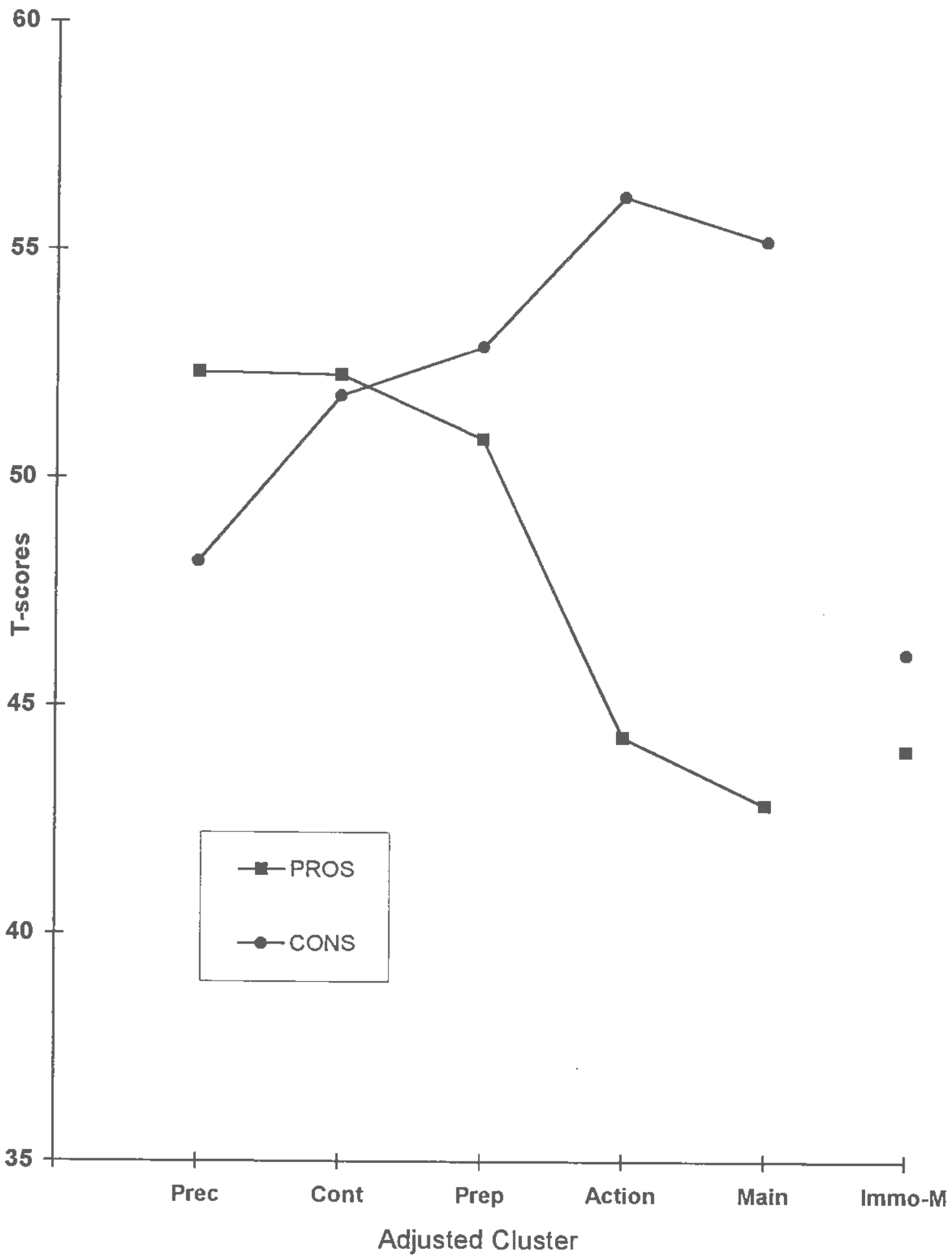

Note: ANOVA for Cons was significant $(F(4,324)=8.36, p<.05)$.

Tukey tests: $\mathrm{PC}<$ Action, Maintenance 


\section{BIBLIOGRAPHY}

Allen, S. J. \& Hubbard, R. (1986). Regression equations for the latent roots of random data correlation matrices with unities on the diagonal. Multivariate Behavioral Research, 21, 393-398.

American Psychiatric Association. (1987). Diagnostic and statistical manual of mental disorders (3rd ed.). Washington, DC: American Psychiatric Association.

Babor, T. F., Kranzler, H. R., \& Lauerman, R. J. (1987), Social drinking as a health and psychosocial risk factor: Anstie's limit revisited. In M. Galanter (Ed.), Recent developements in alcoholism (pp. 373-402). New York: Plenum.

Baer, J. S., Stacy, A., \& Larimer, M. (1991). Biases in the perception of drinking norms among college students. Journal of Studies on Alcohol, 52, 580-586.

Baer, J. S., Marlatt, G. A., Kivlahan, D. R., Fromme, K., Larimer, M. E., \& Williams, E. (1992). An experimental test of three methods of alcohol risk reduction with young adults. Journal of Consulting and Clinical Psychology, 60, 974-979.

Bandura, A. (1977). Self-efficacy: Toward a unifying theory of behavioral change. Psychological Review, $\underline{84}, 191-215$.

Bandura, A. (1982). Self-efficacy: Mechanism in human agency. American Psychologist, 37, $122-147$.

Bentler, P. M. (1989). EOS:Structural equations program manual. Los Angeles, California: BMDP Statistical Software.

Berkowitz, A. D. \& Perkins, H. W. (1986). Problem drinking among college students: A review of recent research. College Health, $\underline{35}, 21-28$.

Blais, L. M. \& Rossi, J. S. (1992). Stages of change: Comparing clusters across four health behaviors. Paper submitted to the 100th convention of the American Psychological Association in Washington, DC 
Brown, S. A. (1985). Expectancies versus background in the prediction of college drinking patterns. Journal of Consulting and Clinical Psychology, 53, 123-130.

Brown, S. A., Goldman, M. S., Inn, A., \& Anderson, L. R. (1980). Expectations of reinforcement from alcohol: Their domain and relation to drinking patterns. Journal of Consulting and Clinical Psychology, 48, 419-426.

Calinski, T. \& Harabasz, J. (1974). A dendrite method for cluster analysis. Communications in Statistics, 3 , 1-27.

Cannon, D. S., Leeka, J. K., Patterson, E. T., \& Baker, T. B. (1990). Principal component analysis of the inventory of drinking situations: Empirical categories of drinking by alcoholics. Addictive Behaviors, 15 , 265-269.

Cattell, R. B. (1966). The Scree test for the number of factors. Multivariate Behavioral Research, 1, 245-276.

Chickering, A. W. (1969). Education and identity. San Francisco: Josset-Bass.

Clark, M. M., Abrams, D. B., Niaura, R. S., Eaton, C. A., \& Rossi, J. S. (1991). Selfefficacy in weight management. Journal of Consulting and Clinical Psychology, 59 , 739-744.

Comrey, A. L. (1988). Factor-analytic methods of scale development in personality and clinical psychology. Journal of Consulting and Clinical Psychology, 56, 754-761.

Dawson, D. A. \& Archer, L. (1992). Gender differences in alcohol consumption: Effects of measurement. British Journal of the Addictions, 87, 119-123.

DiClemente, C. C. (1986). Self-efficacy and the addictive behaviors. Journal of Social and Clinical Psychology, 4, 302-315.

DiClemente, C. C. \& Hughes, S. O. (1990). Stages of change profiles in outpatient alcoholism treatment. Journal of Substance Abuse, 2, 217-235.

DiClemente, C. C., Prochaska, J. O., \& Gilbertini, M. (1985). Self-efficacy and the stages of self change of smoking. Cognitive Therapy and Research, $\underline{9}, 181-200$. 
DiClemente, C. C., Prochaska, J. O., Fairhurst, S. K., Velicer, W. F., Velasquez, M. M., \& Rossi, J. S. (1991). The process of smoking cessation: An analysis of precontemplation, contemplation and preparation stages of change. Journal of Consulting and Clinical Psychology, 59, 295-304.

Elder, J. P., De Moor, C., Young, R. L., Wildey, C. A., Molgaard, C. A., Golbeck, A. L., Sallis, J. F., \& Stern, R. A. (1990). Stages of adolescent tobacco-use acquisition. Addictive Behaviors, $\underline{15}, 449-454$.

Engs, R. (1977). Drinking patterns and drinking problems of college students. Journal of Studies on Alcohol, $38,2144-2156$.

Engs, R. C. \& Hanson, D. J. (1986). Age-specific alcohol prohibition and college students" drinking problems. Psychological Reports, 59, 979-984.

Fillmore, K. M. (1974). Drinking and problem drinking in early adulthood and middle age. Quarterly Journal of Studies on Alcohol, 35, 819-840.

Fillmore, K. M. \& Midanik, L. (1984). Chronicity of drinking problems among men: A longitudinal study. Journal of Studies on Alcohol, $\underline{45}, 228-236$.

Fingarette, H. (1988). Heavy Drinking: The myth of alcoholism as a disease. Berkeley, CA: University of California Press.

Foxcroft, D. R. \& Lowe, G. (1993). Self-attributions for alcohol use in older teenagers. Addiction Research, 1, 1-19.

Frezza, M., de Padova, C., Pozzato, G., Terpin, M., Baraona, E., \& Lieber, C. S. (1990). High blood alcohol levels in women: The role of decreased gastric alcohol dehydrogenase activity and first-pass metabolism. New England Journal of Medicine, $\underline{322}, 127-129$.

Golding, J. M., Burnam, M. A., Benjamin, B., \& Wells, K. B. (1991, August). Reasons for drinking and risk for alcohol abuse or dependence. Poster presented at the annual meeting of the American Psychological Association, San Francisco, CA 
Harford, T. C. (1984). Situational factors in drinking: A developmental perspective on drinking contexts. In P. M. Miller \& T. D. Nirenberg (Eds.), Prevention of alcohol abuse (pp. 119-156). New York: Plenum Press.

Horn, J. L. (1965). A rationale and test for the number of factors in factor analysis. Psychometrika, 30, 179-185.

Jackson, D. N. (1970). A sequential system for personality scale development. In Spielberger (Ed.), Current topics in clinical and community psychology (Vol. 2, pp. 61-96). New York: Academic Press.

Jackson, D. N. (1971). The dynamics of structured personality tests: 1971 . Psychological Review, $\underline{78}, 229-248$.

Janis, I. L. \& Mann, L. (1968). A conflict-theory approach to attitude change and decision making. In A. Greenwald, T. Brook, \& T. Ostrom (Eds.), Psychological foundation of attitudes (pp. 327-360). New York: Academic Press.

Janis, I. L. \& Mann, L. (1977). Decision making: A psychological analysis of conflict. choice and commitment. New York: Free Press.

Jessor, R. (1987). Problem-behavior theory, psychosocial development, and adolescent

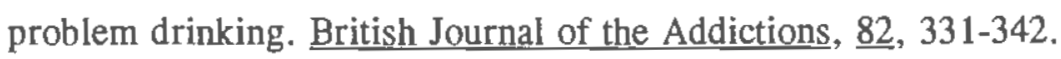

Johnston, J. D., O'Malley, P. M., \& Bachman, J. G. (1992). Smoking. drinking and illicit drug use among American secondary school students, college students, and young adults, 1975-1991 (Vol. 2). Washington, D.C.: National Institute on Drug Abuse.

Jones, M. C. (1968). Personality correlates and antecedents of drinking patterns in adult males. Journal of Consulting and Clinical Psychology, 31, 1-12.

Jones, M. C. (1971). Personality antecedents and correlates of drinking patterns in women. Journal of Consulting and Clinical Psychology, 주, 61-69.

Kraft, D. P. (1988). The prevention and treatment of alcohol problems on a college campus. Journal of Alcohol and Drug Education, 34, 37-51. 
Laforge, R. G., Rossi, J. S., \& Migneault, J. P. (1994, April). Predicting intention to change high risk drinking practices. Poster presented at the 15th annual conference of the Society of Behavioral Medicine, Boston, MA

Lautenschlager, G. J. (1989). A comparison of alternatives to conducting Monte Carlo analyses for determining parallel analysis criteria. Multivariate Behavioral Research, $\underline{24}, 365-395$.

MacAndrew, C. \& Edgerton, R. B. (1969). Drunken comportment: A social explanation. Chicago: Aldine.

Marcus, B. H., Rossi, J. S., Selby, V. C., Niaura, R. S., \& Abrams, D. B. (1992). The Stages and Processes of Exercise Adoption and Maintenance in a Worksite Sample. Health Psychology, 11, 386-395.

Marcus, B. H., Selby, V. C., Niaura, R. S., \& Rossi, J. S. (1992). Self-efficacy and the stages of exercise behavior change. Research Quarterly for exercise and sport, $\underline{63}, 60$ 66.

Marlatt, G. A. \& Gordon, J. R. (1980). Determinants of relapse: Implication for the maintenance of behavior change. In P. Davidson \& S. Davidson (Eds.), Behavioral medicine: Changing health lifestyles (pp. 410-452), New York: Brunner/Mazel.

Marlatt, G. A. \& Gordon, J. R. (1985). Relapse prevention: Maintenance strategies in the treatment of addictive behaviors. New York: Guilford Press.

Marlatt, G. A. \& Rohsenow, D. J. (1980). Cognitive processes in alcohol use: Expectancy and the balanced placebo design. In N. K. Mello (Ed.), Advances in substance abuse: Behavioral and biological research (pp. 159-199). Greenwich, CT: JAI Press.

Mayfield, D., McLeod, G., \& Hall, P. (1974). The CAGE questionnaire: Validation of a new alcoholism screening instrument. American Journal of Psychiatry, 131, 1121-1123.

McConnaughy, E. A., DiClemente, C. C., Prochaska, J. O., \& Velicer, W. F. (1989). Stages of change in psychotherapy: A follow-up report. Psychotherapy, 26, 494-503. 
McConnaughy, E. A., Prochaska, J. O., \& Velicer, W. F. (1982). Stages of change in psychotherapy: Measurement and sample profiles. Psychotherapy: Theory, Research and Practice, 20, 368-375.

Meilman, P. W., Stone, J. E., Gaylor, M. S., \& Turco, J. H. (1990). Alcohol consumption by college undergraduates: Current use and 10-year trends. Journal of Studies on Alcohol, 51, 389-395.

Migneault, J. P. (1992). A validation study of stages of change for risky drinking.

Unpublished master's thesis, University of Rhode Island, Kingston, RI.

Migneault, J. P., Pallonen, U. E., \& Velicer, W. F. (1994, April). Stage and decisional balance for adolescent alcohol use. Paper submitted for presentation at the 15 th annual meeting of the Society of Behavioral Medicine, Boston, MA

Migneault, J. P., Stevenson, J. F., \& Velicer, W. F. (1994, April). A validation study of the stages of change for risky drinking in college. Poster presented at the 15th annual meeting of the Society of Behavioral Medicine in Boston, MA

Miller, W. R. (1987). Techniques to modify hazardous drinking patterns. In M. Galanter (Ed.), Recent developments in alcoholism (Vol. 5, pp. 425-438). New York: Plenum. Milligan, G. W. (1980). An examination of the effect of six types of error perturbation on fifteen clustering algorithms. Psychometrika, 45, 325-342.

Milligan, G. W. \& Cooper, M. C. (1985). An examination of procedures for determining the number of clusters in a data set. Psychometrika, $\underline{50}, 159-179$.

Moskowitz, J. M. (1989). The primary prevention of alcohol problems: A critical review of the research Literature. Journal of Studies on Alcohol, 50, 54-88.

Mulaik, S. A., James, L. R., Van Alstine, J., Bennett, N., Lind, S., \& Stilwell, C. D. (1988). Evaluation of goodness-of-fit indices for structural equation models. Psychological Bulletin, 105, 430-445.

Pallonen, U. E., Murray, D. M., Schmid, L., Pirie, P., \& Luepker, R. (1990). Patterns of self-initiated smoking cessation among young adults. Health Psychology, $\underline{9}, 418-426$. 
Pallonen, U. E., Velicer, W. F., Bellis, J. M., Tsoh, J., \& Smith, N. F. (1994, April). $\underline{A}$ computer-based expert system for smoking prevention and cessation in adolescents. Paper presented at the 15 th annual meeting of the Society of Behavioral Medicine in Boston, MA

Peele, S. (1993). The conflict between public health goals and the temperance mentality.

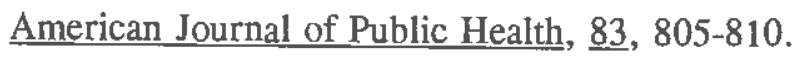

Perry, C. L., Baranowski, T., \& Parcel, G. S. (1990). How individuals, environments, and health behavior interact: Social learning theory. In K. Glanz, F. M. Lewis, \& B. K. Rimer (Eds.), Health behavior and health education: Theory, research, and practice (pp. 161-186). San Francisco: Jossey-Bass Inc.

Prochaska, J. O. (1979). Systems of psychotherapy: A transtheoretical analysis (2nd ed.). Homewood, Illinois: Dorsey Press.

Prochaska, J. O. (1994). Strong and Weak Principles for Progressing from Precontemplation to Action Based on Twelve Problem Behaviors. Health Psychology, 13, 47-51.

Prochaska, J. O. \& DiClemente, C. C. (1983). Stages and processes of self-change of smoking: Toward an integrative model of change. Journal of Consulting and Clinical Psychology, 51, 390-395.

Prochaska, J. \& DiClemente, C. (1984). The transtheoretical approach: Crossing the traditional boundaries of therapy. Homewood, Illinois: Dow-Jones/Irwin.

Prochaska, J. O. \& DiClemente, C. C. (1985). Common processes of self-change in smoking, weight control and psychological distress. In S. Shiffman \& T. Wills (Eds.), Coping and substance use: A conceptual framework (pp. 345-363). New York: Academic Press.

Prochaska, J. O., DiClemente, C. C., Velicer, W. F., Ginpil, S., \& Norcross, J. C. (1985). Predicting change in smoking status for self-changers. Addictive Behaviors, 10, 395406. 
Prochaska, J. O., DiClemente, C. C., Velicer, W. F., \& Rossi, J. S. (1993). Standardized, individualized, interactive and personalized self-help programs for stages of smoking cessation. Health_Psychology, 12, 399-405.

Prochaska, J. O. \& Norcross, J. C. (1994). Systems of psychotherapy: A transtheoretical analysis (3rd ed.). Pacific Grove, CA: Brooks/Cole Publishing Co.

Prochaska, J. O., Norcross, J. C., Fowler, J. L., Follick, M. J., \& Abrams, D. B. (1992). Attendance \& outcome in a worksite weight control program: Processes and stages of change as process and predictor variables. Addictive Behaviors, 17, 35-45.

Prochaska, J. O., Redding, C. A., Harlow, L. L., Rossi, J. S., \& Velicer, W. F. (in press). The transtheoretical model of change and HIV prevention. Health Education Quarterly.

Prochaska, J. O., Rossi, J. S., \& Snow, M. G. (1992). Processes of Change in Alcoholics Anonymous: Maintenance Factors in Long-Term Sobriety. Journal of Studies on Alcohol.

Prochaska, J. O., Rossi, J. S., \& Wilcox, N. S. (1991). Change processes and psychotherapy outcomes in integrative case research. Journal of Psychotherapy Integration, 1(2), 103-119.

Prochaska, J. O., Velicer, W. F., DiClemente, C. C., \& Fava, J. (1988). Measuring processes of change: Applications to the cessation of smoking. Journal of Consulting and Clinical Psychology, 56, 520-528.

Prochaska, J. O., Velicer, W. F., Guadagnoli, E., Rossi, J. S., \& DiClemente, C. C. (1991). Patterns of change: Dynamic typology applied to smoking cessation. Multivariate Behavioral Research, 26, 83-107.

Prochaska, J. O., Harlow, L. L., Redding, C. A., Snow, M. G., Rossi, J. S., \& Velicer, W. F. (1990). Models of Behavior Change for Condom Use: Preliminary Analyses in an AIDS-risking Community Sample. Technical report to the Centers of Disease COntroll, contract grant \#0-4115-002 
Prochaska, J. O., Velicer, W. F., Rossi, J. S., Goldstein, M. G., Marcus, B. H., Rakowski, W., Fiore, C., Harlow, L. L., Redding, C. A., Rosenbloom, D., \& Rossi, S. R. (1994). Stages of change and decisional balance for twelve problem behaviors. Health Psychology, 13, 39-46.

Redding, C. A. (1993). The transtheoretical model applied to safer sex behavior among university students: A cross-sectional investigation. Unpublished doctoral dissertation, University of Rhode Island, Kingston, RI.

Reed, G. R. (1993). The transtheoretical model and exercise behavior: A comparison of five staging methods. Unpublished master's thesis, University of Rhode Island, Kingston, RI.

Rollnick, S., Heather, N., Gold, R., \& Hall, W. (1992). Development of a short 'readiness to change' questionnaire for use in brief, opportunistic interventions among excessive drinkers. British Journal of the Addictions, 87, 743-754.

Rosenbloom, D. (1991). A transtheoretical analysis of change among cocaine users. Unpublished doctoral Dissertation, University of Rhode Island, Kingston RI.

Rossi, J. S. (1989). Exploring behavioral approaches to UV risk reduction. In A. Moshell \& L. W. Blankenbaker (Eds.), Sunlight, ultraviolet radiation, and the skin (pp. 91-93). Bethesda, MD: National Institute of Health.

Rossi, J. S. (1992, August). Common process of change across nine problem behaviors.

Paper presented at the 100th convention of the American Psychological Association in Washington, DC

Rossi, J. S., Prochaska, J. O., \& DiClemente, C. C. (1988). Processes of change in heavy and light smokers. Journal of Substance Abuse, 1, 1-9.

Rossi, S. R. (1993). Application of the Transtheoretical Model of behavior change to dietary fat reduction in a naturalistic population. Unpublished doctoral dissertation, University of Rhode Island, Kingston, RI. 
Rossi, S. R. \& Rossi, J. S. (1993, March). Processes of Change for Dietary Fat Reduction.

Paper presented at the 13 th annual convention of the Society for Behavioral Medicine, San Francisco, CA

Rossi, S. R., Rossi, J. S., Prochaska, J. O., \& Velicer, W. F. (1992). Application of the Transtheoretical Model of Behavior Change to Dietary Fat, (Abstract). International Journal of Psychology, 27, 628.

Saunders, J. B. \& Aasland, O. G. (1987). WHO collaborative project on the identification and treatment of persons with harmful alcohol consumption: Repert on Phase I: The development of a screening device (Vol. WHO Offset Publication 86.3). Geneva: World Health Organization.

Science. (1987). Is alcoholism treatment effective? , 236, 20-22.

Searle, W. S. (1983). Cubic clustering criterion (Tech. Rep. A-108). Cary, NC: SAS Institute.

Shaffer, H. J. (1992). The psychology of stage change: The transition from addiction to recovery. In J. H. Lowinson, P. Ruiz, R. B. Millman, \& J. G. Langrod (Eds.), Substance abuse: A comprehensive textbook (2nd ed., pp. 100-105). Baltimore: Willians \& Wilkins.

Shedler, J. \& Block, J. (1990). Adolescent drug use and psychological health. American Psychologist, $45,612-630$.

Snow, M. G. (1991). A transtheoretical analysis of strategies in the recovery process from alcohol problems. Unpublished doctoral dissertation, University of Rhode Island, Kingston, RI.

Stern, R. A., Prochaska, J. O., Velicer, W. F., \& Elder, J. P. (1987). Stages of adolescent cigarette smoking acquisition: Measurement and sample profiles. Addictive Behaviors, $12,319-329$.

Stevenson, J., Migneault, J. P., \& Mitchell, R. (1990, November). The University of Rhode Island alcohol-drug health survey: Final report. Unpublished report 
Tsoh, J. Y. (1993). Motivation and stages of change among drug addicts in drug abuse treatment programs. Unpublished master's thesis, University of Rhode Island, Kingston, RI.

Vaillant, G. E. (1977). Adaptation to Life. Boston: Little, Brown and Company.

Velicer, W. F. (1976). Determining the number of components from the matrix of partial correlations. Psychometrika, 41, 321-327.

Velicer, W. F., DiClemente, C. C., Prochaska, J. O., \& Brandenburg, N. (1985), A decisional balance measure for assessing and predicting smoking status. $\underline{\text { Journal of }}$ Personality and Social Psychology, 48, 1279-1289.

Velicer, W. F., DiClemente, C. C., Rossi, J. S., \& Prochaska, J. O. (1990). Relapse situations and self-efficacy: An integrative model. Addictive Behaviors, 15, 271-283.

Velicer, W. F., Hughes, S. L., Fava, J. L., Prochaska, J. O., \& DiClemente, C. C. (submitted for review). An empirical typology of subjects within stage of change. . Velicer, W. F., Prochaska, J. O., Bellis, J. M., Rossi, J. S., Fava, J. L., \& Steiger, J. H. (1993). An expert system intervention for smoking cessation. Journal of the Applied Psychology, 18, 269-290.

Wechsler, H., Davenport, A., Dowdall, G., Moeykens, B., \& Castillo, S. (1994). Health and behavioral consequences of binge drinking in college. Journal of the American Medical Association, 272, 1672-1677.

Wechsler, H. \& McFadden, M. (1979). Drinking among college students in New England. Journal of Studies on Alcohol, 40, 969-996.

Werch, C. E. (1990). Behavioral self-control strategies for deliberately limiting drinking among college students. Addictive Behaviors, 15, 119-128.

White, H. R. \& Labouvie, E. W. (1989). Towards the assessment of adolescent problem

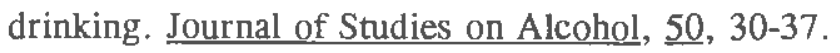

Williams, G. D. \& Debakey, S. F. (1992). Changes in levels of alcohol consumption: United States, 1983-1988. British Journal of the Addictions, 87, 643-648. 
Winston, R. B. \& Miller, T. K. (1987). Student developmental task and lifestyle inventory manual. Athens, GA: Student Development Associates.

Zinberg, N. E. (1981). Alcohol addiction: Toward a more comprehensive definition. In M. H. Bean \& N. E. Zinberg (Eds.), Dynamic approaches to the understanding and treatment of alcoholism. New York: The Free Press.

Zinberg, N. E. \& Fraser, K. M. (1979). The role of social setting in the prevention and treatment of alcoholism. In J. H. Mendelson \& N. K. Mello (Eds.), The diagnosis and treatment of alcoholism (pp. 457-483). New York: McGraw-Hill.

Zinberg, N. E., Harding, W. M., \& Winkeller, M. (1977). A study of social regulatory mechanisms in controlled illicit drug users. Journal of Drug Issues, 7, 117-133.

Zwick, W. R. \& Velicer, W. F. (1986). Comparison of five rules for determining the number of components to retain. Psychological Bulletin, $99,432-442$. 